\title{
Yang-Mills Measure on Compact Surfaces
}

\author{
Thierry LEVY \\ Statistical Laboratory, Cambridge, UK \\ t.levy@statslab.cam.ac.uk
}

January 2001 



\section{Abstract}

We construct and study the Yang-Mills measure in two dimensions. According to the informal description given by the physicists, it is a probability measure on the space of connections modulo gauge transformations on a principal bundle with compact structure group. We are interested in the case where the base space of this bundle is a compact orientable surface.

The construction of the measure in a discrete setting, where the base space of the fiber bundle is replaced by a graph traced on a surface, is quite well understood thanks to the work of E. Witten. In contrast, the continuum limit of this construction, which should allow to put a genuine manifold as base space, still remains problematic.

This work presents a complete and unified approach of the discrete theory and of its continuum limit. We give a geometrically consistent definition of the Yang-Mills measure, under the form of a random holonomy along a wide, intrinsic and natural class of loops. This definition allows us to study combinatorial properties of the measure, like its Markovian behaviour under the surgery of surfaces, as well as properties specific to the continuous setting, for example, some of its microscopic properties. In particular, we clarify the links between the Yang-Mills measure and the white noise and show that there is a major difference between the Abelian and semi-simple theories. We prove that it is possible to construct a white noise using the measure as a starting point and vice versa in the Abelian case but we show a result of asymptotic inde-

pendence in the semi-simple case which suggests that it is impossible to extract a white noise from the measure. 



\section{Contents}

$\begin{array}{ll}\text { Introduction } & \text { ix }\end{array}$

$\begin{array}{lll}1 & \text { Discrete Yang-Mills measure } & 1\end{array}$

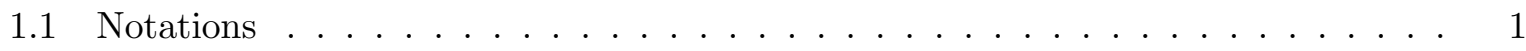

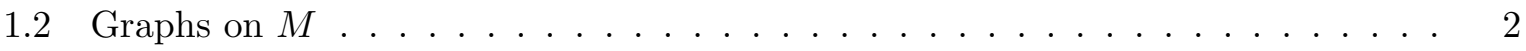

1.2 .1 Pregraphs . . . . . . . . . . . . . . . . . . . . 2

1.2 .2 Graphs $\ldots \ldots \ldots \ldots \ldots \ldots \ldots$

1.3 Discrete holonomy and gauge transformations . . . . . . . . . . . . . . . . 4

1.4 Discrete Yang-Mills measure . . . . . . . . . . . . . . . . . . . . . . 6

1.5 Conditional Yang-Mills measure . . . . . . . . . . . . . . . . . . . . 6

1.5.1 Conditional Haar measure $\ldots \ldots \ldots \ldots$. . . . . . . . . . 7

1.5.2 Conditional Yang-Mills measure . . . . . . . . . . . . . . . 7

1.5 .3 Gauge transformations . . . . . . . . . . . . . . . . . . . . . . . . . . . . . . . . . . .

1.6 Invariance by subdivision $\ldots \ldots \ldots \ldots \ldots \ldots$

1.7 Invariance by area-preserving diffeomorphisms . . . . . . . . . . . . . . . . . . . . . . . . . . . . . . . . .

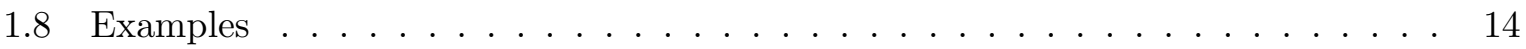

1.8 .1 Holonomy along an open path . . . . . . . . . . . . . . . 14

1.8 .2 Holonomy along the boundary of a small disk . . . . . . . . . . . . . . . . 15

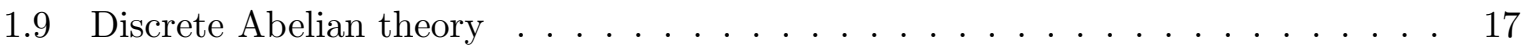

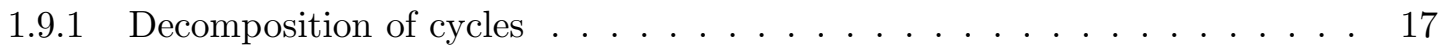

$1.9 .2 \quad$ Study of a fundamental system . . . . . . . . . . . . . . . . . . 18

1.9 .3 Gaussian aspect of the Abelian theory . . . . . . . . . . . . . . . . . . 20

1.9 .4 The double layer potential . . . . . . . . . . . . . . . . 22

2 Continuous Yang-Mills measure $\quad 25$

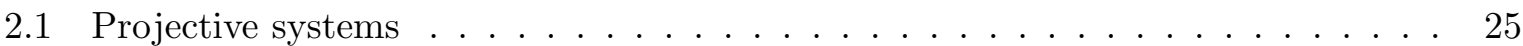

2.2 Piecewise geodesic graphs $\ldots \ldots \ldots \ldots \ldots \ldots \ldots$. . . . . . . . . . . . . . . 26

2.3 Preliminary results $\ldots \ldots \ldots \ldots \ldots \ldots \ldots \ldots$

2.3 .1 Lassos . . . . . . . . . . . . . . . . . . . . . . . 29

2.3 .2 Holonomy along small piecewise geodesic loops . . . . . . . . . . . . . 30

$2.3 .3 \quad$ Double layer potential of small piecewise geodesic loops . . . . . . . . . . 32

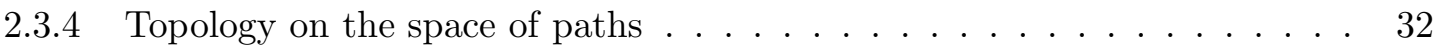

2.4 Approximation of embedded paths . . . . . . . . . . . . . . . . . 33

2.4 .1 Tubular neighbourhoods and Fermi coordinates . . . . . . . . . . . . . . . 34

2.4 .2 Piecewise geodesic approximation . . . . . . . . . . . . . . . . . 34 
$2.5 \quad$ Random holonomy along embedded paths . . . . . . . . . . . . . . . . . . 35

2.5.1 Existence of a limit random holonomy $\ldots \ldots \ldots \ldots$. . . . . . . . . 35

2.5 .2 Unicity of the limit random holonomy $\ldots \ldots \ldots \ldots$. . . . . . . . . . . . . . . . . . . . . . 36

2.5 .3 Continuity of the double layer potential (1) . . . . . . . . . . . . 38

2.6 Random holonomy along arbitrary paths . . . . . . . . . . . . . . . . . 38

2.6 .1 Construction of the random holonomy . . . . . . . . . . . . . . 38

2.6 .2 Continuity of the random holonomy $\ldots \ldots \ldots \ldots$. . . . . . . . . 39

2.6 .3 Continuity of the double layer potential (2) . . . . . . . . . . . . . . 40

2.7 Law of the random holonomy $\ldots \ldots \ldots \ldots \ldots$. . . . . . . . . . . . . . . . . . . . . . . . . .

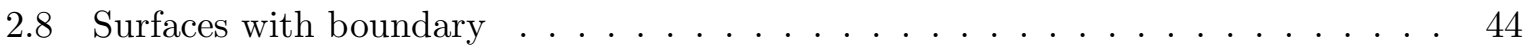

$2.8 .1 \quad$ Natural law of the holonomy along the boundary . . . . . . . . . . . . . 44

$2.8 .2 \quad$ Definition of the random holonomy . . . . . . . . . . . . . . . 45

2.9 Summary of the properties of the random holonomy $\ldots \ldots \ldots$. . . . . . . . 46

$2.9 .1 \quad$ Existence, unicity in law and main properties . . . . . . . . . . . . . 46

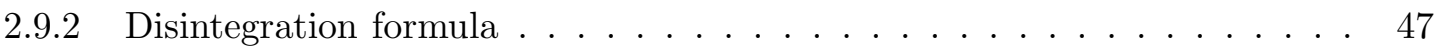

2.10 Yang-Mills measure . . . . . . . . . . . . . . . . . . . . 47

2.10 .1 Definition of the Yang-Mills measure . . . . . . . . . . . . . . . . 47

2.10 .2 Regularity properties . . . . . . . . . . . . . . . . . . 50

2.10 .3 Remarkable subfamilies of random variables . . . . . . . . . . . . . . . 51

\begin{tabular}{lll}
\hline Abelian theory & 53
\end{tabular}

3.1 The random holonomy as a white noise functiona $\ldots \ldots \ldots \ldots$. . . . . . . 53

3.1 .1 Definition of the white noise functional . . . . . . . . . . . . . 53

3.1 .2 Regularity of the new random holonomy . . . . . . . . . . . . . . . . . . 54

3.1 .3 Identification of the random holonomies $\ldots \ldots \ldots \ldots$. . . . . . . 57

3.2 Small scale structure of the Yang-Mills field . . . . . . . . . . . . . . . . . 58

$3.2 .1 \quad$ Extraction of a white noise $\ldots \ldots \ldots \ldots \ldots \ldots$. . . . . . . . . . . . . . . . . . . . . . . .

3.2 .2 Meaning of the variable $T$. . . . . . . . . . . . . . . 61

$3.3 \quad$ Square-integrability of the double-layer potential . . . . . . . . . . . . . . 62

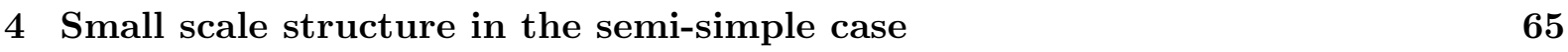

4.1 Statement of a zero-one law . . . . . . . . . . . . . . . . . . 65

4.2 Proof of the zero-one law . . . . . . . . . . . . . . . . . 66

4.2 .1 Computation of the conditional expectation . . . . . . . . . . . . 66

$4.2 .2 \quad$ Characters of a semi-simple Lie group $\ldots \ldots \ldots$. . . . . . . . . . 67

$4.2 .3 \quad$ Character computations . . . . . . . . . . . . . . . . . . 69

$4.2 .4 \quad$ Zero-one law on the pland $\ldots \ldots \ldots \ldots$. . . . . . . . . . 70

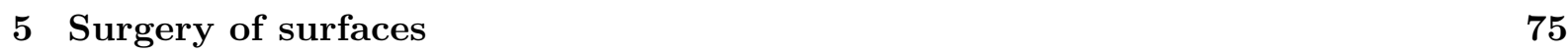

$5.1 \quad$ Markov property of the Yang-Mills field $\ldots \ldots \ldots \ldots \ldots$. . . . . . . . . . . . . . . . .

$5.2 \quad$ Study of an example . . . . . . . . . . . . . . . . . . . 78

$5.3 \quad$ Sewing of two surfaces along one circle $\ldots \ldots \ldots \ldots$. . . . . . . . . 80

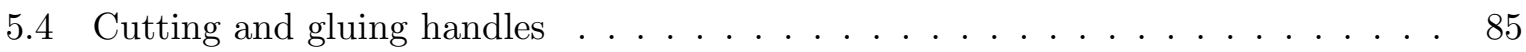

5.5 Conditional partition functions . . . . . . . . . . . . . . . . . . 90

$5.5 .1 \quad$ Algebraic properties of the partition functions . . . . . . . . . . . . . . . 90

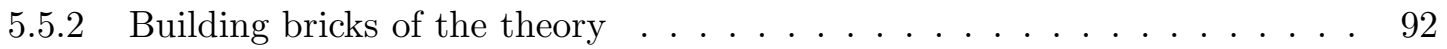


5.5 .3 Transition fonctions of the Markov field $\ldots \ldots \ldots$. . . . . . . . . . . 95 



\section{Introduction}

This thesis is devoted to the construction and to the study of the Yang-Mills measure in two dimensions. The quadridimensional and pseudo-Riemannian equivalent of this measure is used by physicists in gauge theories such as quantum electrodynamics and quantum chromodynamics, in order to describe the fundamental interactions. It appears in path integrals, which are known to be often ill defined. Physicists describe the Yang-Mills measure as a probability measure on the space of connections modulo gauge transformations on a principal bundle with compact structure group. We are interested in the case where the base space of this bundle is a compact orientable surface. The informal expression of the measure is the following:

$$
d \mu(\omega)=\frac{1}{Z} e^{-\frac{1}{2} S(\omega)} D \omega
$$

where $S$ is the Yang-Mills action, that is, the $L^{2}$ norm of the curvature. The constant $Z$ is a normalization constant and the measure $D \omega$ should be a translation invariant measure on the space of connections.

The construction of the measure in a discrete setting, where the base space of the fiber bundle is replaced by a graph traced on a surface, is quite well understood thanks to the work of Witten Wi. In contrast, the continuum limit of this construction, which should allow to put a genuine manifold as base space, still remains problematic. Several works in this direction have led to substantial progress but not yet to an entirely satisfactory solution.

This work presents a complete and unified approach of the discrete theory and of its continuum limit. We give a geometrically consistent definition of the Yang-Mills measure, under the form of a random holonomy along a wide, intrinsic and natural class of loops. This definition allows us to study combinatorial properties of the measure, like its Markovian behaviour under the surgery of surfaces, as well as properties specific to the continuous setting, for example, some of its microscopic properties. In particular, we clarify the links between the Yang-Mills measure and the white noise and show that there is a major difference between the Abelian and semi-simple theories. We prove that it is possible to construct a white noise using the measure as a starting point and vice versa in the Abelian case but we show a result of asymptotic independence in the semi-simple case which suggests that it is impossible to extract a white noise from the measure.

\section{Statement of the problem.}

We are given a surface $M$, a Lie group $G$ and a principal bundle $P$ over $M$. The surface $M$ is a differentiable two-dimensional compact orientable manifold, with or without boundary. It 
is endowed with a measure $\sigma$ which is equivalent to the Lebesgue measure in any chart, with a positive smooth density. The group $G$ is a compact connected Lie group. In most examples, it will be either Abelian or semi-simple. The fiber bundle $P$ is a principal fiber bundle over $M$ with structure group $G$.

Recall that a connection on $P$ is a $G$-invariant choice of a horizontal distribution in $P$ and that this choice can be represented by a $\mathfrak{g}$-valued 1 -form $\omega$ on $P$, where $\mathfrak{g}$ is the Lie algebra of $G$. The curvature of the connection $\omega$ is the $\mathfrak{g}$-valued 2 -form $\Omega$ on $P$ defined by

$$
\Omega(X, Y)=d \omega(X, Y)+[\omega(X), \omega(Y)] .
$$

The curvature can be considered an ad $P$-valued 2 -form on $M$, where ad $P$ is the fibre bundle associated with $P$ by the adjoint representation of $G$ on $\mathfrak{g}$. If we choose an orientation on $M, \Omega$ can be identified with a section of ad $P$. An ad-invariant scalar product on $\mathfrak{g}$ allows to define a metric on ad $P$ and hence the norm $\|\Omega\|$ of the curvature. This norm does not depend on the choice of the orientation of $M$ and the Yang-Mills action is defined on the space $\mathcal{A}$ of connections on $P$ by

$$
\begin{aligned}
S: \mathcal{A} & \longrightarrow \mathbf{R}_{+} \\
\omega & \longmapsto S(\omega)=\int_{M}\|\Omega\|^{2} d \sigma .
\end{aligned}
$$

Our aim is to give a sense to the informal expression (11). The first problem is of course that there is no translation invariant measure on the infinite dimensional affine space $\mathcal{A}$. Another one is the invariance of the action $S$ under the action of a huge group, that of gauge transformations of $P$. This group, denoted by $\mathcal{J}$, is the group of diffeomorphisms of $P$ over the identity of $M$ that commute with the action of $G$. It acts by pull-back on $\mathcal{A}$ and preserves $S$, since it acts on the curvature by pointwise conjugation, which does not change the norm in ad $P$. Because of this invariance, the constant $Z$ should be proportional to the volume of $\mathcal{J}$, hence be infinite. To avoid this problem, we try to construct the measure on the quotient space $\mathcal{A} / \mathcal{J}$ instead of $\mathcal{A}$. This means that we will be able to integrate only gauge-invariant functions against the Yang-Mills measure, in agreement with the physical principle saying that observable quantities must be gauge-invariant. On the other hand, this quotient space has a much more complicated structure than an affine space. This is why one usually tries to avoid to work directly on it and prefer to work on a function space, as we explain below. The work of D. Fine [Fi1, Fi2] is an exception from this point of view, since the author analyzes the geometrical structure of $\mathcal{A} / \mathcal{J}$ in order to give sense to (11).

\section{The Yang-Mills measure as random holonomy.}

A starting point may be to ask what functions we want to be able to integrate against the YangMills measure. Physicists' answer this question is that we must be able to integrate Wilson loops.

A connection $\omega$ on $P$ defines a parallel transport along regular paths on $M$. The parallel transport along a given path $c:[0,1] \longrightarrow M$ is a $G$-equivariant diffeomorphism of the fiber $P_{c(0)}$ into the fiber $P_{c(1)}$, denoted by $\operatorname{hol}(\omega, c)$. If $c$ is a loop and if we fix a point $p$ in the fiber $P_{c(0)}$, this diffeomorphism can be represented by the element $g$ of $G$ such that $\operatorname{hol}(\omega, c)(p)=p g$. If we choose another point in $P_{c(0)}$, we find another element of $G$ conjugate to $g$. So, for any 
representation $\rho$ of $G$ and any loop $l$, one defines the Wilson loop $W_{l, \rho}$ by

$$
W_{l, \rho}(\omega)=\operatorname{tr} \rho(\operatorname{hol}(\omega, l)) .
$$

The functions that we want to integrate are central functions of the holonomy along loops. We just noticed that the holonomy along a loop determines a conjugacy class in $G$. We must also take into account the action of the gauge group, which conjugates by the same element of $G$ the holonomies along all loops based at the same point. Let $L M$ denote the set of regular paths on $M$ and $\mathcal{F}(M, G), \mathcal{F}(L M, G)$ the sets of $G$-valued functions on $M, L M$. An element $j$ of the group $\mathcal{F}(M, G)$ acts on an element $f$ of $\mathcal{F}(L M, G)$ by:

$$
j . f(l)=j(l(0))^{-1} f(l) j(l(0)) .
$$

It is possible to define a map from $\mathcal{A} / \mathcal{J}$ into the quotient $\mathcal{F}(L M, G) / \mathcal{F}(M, G)$, mapping a connection to the class of the holonomy that it determines along the elements of $L M$. An argument of Sengupta Se1 proves that this map is injective. Thus, we change our point of view: we seek now a measure on the space $\mathcal{F}(L M, G) / \mathcal{F}(M, G)$, viewing this space as a space of generalized connections modulo gauge transformations. In fact, we shall construct a measure on $\mathcal{F}(L M, G)$ and take the quotient of this measure by $\mathcal{F}(M, G)$. In other words, we really want to construct a random holonomy instead of a random connection. This will be easier because we can use the classical tools of probability to construct a measure on a function space.

At this point, it is necessary to characterize more precisely the Yang-Mills measure. Either one tries to extract more information from the informal expression of the measure or one looks for other description of this measure. The last option is the one that we choosed in this work, using the combinatorial description given by Migdal and Witten. The first one is based on the Gaussian character of the measure and was used by Driver and Sengupta [Dr1, Se1, Se2].

\section{Gaussian interpretation: curvature of the random connection.}

Assume that $G$ is abelian, for example $G=U(1)$. The relation (2) between a connection and its curvature becomes linear. A formal change of variables in (1) gives

$$
d \mu(\Omega)=\frac{1}{Z^{\prime}} e^{-\frac{1}{2}\|\Omega\|^{2}} D \Omega .
$$

Recall that the curvature may be seen as an ad $P$-valued two-form, or as a section of ad $P$ if an orientation of $M$ is fixed. Since $G$ is Abelian, its adjoint representation on its Lie algebra is trivial. Hence, the fiber bundle ad $P$, which is the vector bundle associated to $P$ for this adjoint representation, is trivial, and may therefore be identified with $M \times \mathfrak{g}$. We recognize in (3) the expression of a Gaussian measure on the Hilbert space of square integrable $\mathfrak{g}$-valued functions on $M$. This leads us to the main idea of the interpretation of (1): under the Yang-Mills measure, the random curvature of a connection has a Gaussian distribution, it is a $\mathfrak{g}$-valued white noise on $M$ with intensity $\sigma$.

This argument is of course specific to the abelian case, since in general, $\Omega$ is a quadratic function of $\omega$. Nevertheless, the fact that $M$ is two-dimensional allows to overcome this problem: it is always possible to get back to a situation similar to the abelian case by a gauge fixing procedure. This requires a word of explanation. 
If we choose a local trivialization of $P$ on an open subset $U \subset M$, i.e. a local section $s: U \longrightarrow P$ of $P$, we can pull-back by $s$ all objects living on $P$, in particular the connection and curvature forms. One denotes usually $A=s^{*} \omega$ and $F=s^{*} \Omega$. These forms on $M$ satisfy a structure equation $F=d A+[A, A]$ identical to (2). Let $j$ be an element of the gauge group $\mathcal{J}$. This element can act in two different ways in this situation, either by transforming the section $s$ into $j \circ s$ or by transforming the forms $\omega$ and $\Omega$ into $j^{*} \omega$ and $j^{*} \Omega$. These two ways are indistinguishable from the base space, since $(j \circ s)^{*}=s^{*} j^{*}$. So, we denote without ambiguity $s^{*} j^{*} \omega=(j \circ s)^{*} \omega$ by $A^{j}$.

What is specific to the two-dimensional case is that given a connection, it is possible to choose $s$ in such a way that $[A, A]=0$. Choose $U$ small enough to admit local coordinates $x, y$. Set $m=(0,0)$ in these coordinates and choose $p$ in $P_{m}$. Then define $s$ along the $y$-axis by lifting it horizontally, starting at $p$. Now, starting from each point $\left(0, y_{0}\right)$ in $U$, define $s$ on the line through $\left(0, y_{0}\right)$ parallel to the $x$-axis by lifting it horizontally, starting at $s\left(0, y_{0}\right)$. The section $s$ is smooth and horizontal along all lines parallel to the $x$-axis. Thus, $A=s^{*} \omega$ takes the form $A=A_{y} d y$ and $[A, A]=0$. When one looks at $P$ through $s$, what one sees is similar to the abelian case, up to the fact that the section through which the relation between connection and curvature should be linear depends on the connection.

\section{From the curvature to the holonomy.}

The next step is to define a random holonomy using the random curvature. The method is based on deterministic links between holonomy and curvature. Assume that $G=U(1)$ and take $\mathbf{R}^{2}$ as base space, although it is not a compact surface. Given a connection $\omega$ on the fiber bundle $\mathbf{R}^{2} \times G$ and a simple loop $l$ which bounds a domain $D$, the Stokes formula gives

$$
\operatorname{hol}(\omega, l)=\exp i \oint_{l} A=\exp i \int_{D} d A=\exp i \int_{D} F=\exp i\left(F, \mathbf{1}_{D}\right)_{L^{2}} .
$$

This formulation is easily extended to the random case. Indeed, pick a white noise $W$ on $\mathbf{R}^{2}$, i.e. an isometry from $L^{2}\left(\mathbf{R}^{2}\right)$ into a vector space of Gaussian random variables. One can replace $F$ by $W$ in the last expression and define a random holonomy along $l$ by

$$
H_{l}=\exp i W\left(\mathbf{1}_{D}\right) .
$$

The construction that we present in the abelian case in chapter 3 is an extension of this procedure to surfaces whose topology is non trivial and where the interior of a loop is not well defined.

It is possible here to understand better the difficulties of Driver and Sengupta. They tried to use this method in the case of a non-Abelian structure group. But in this case, the holonomy is not $\exp \int_{l} A$, but $P \exp \oint_{l} A$, which is a compact notation for the solution of the differential equation

$$
\left\{\begin{array}{l}
\dot{h}_{t}=A(\dot{l}(t)) h_{t} \\
h_{0}=1 .
\end{array}\right.
$$

The Stokes formula does not work in this frame. In some sense, one has to choose in which order one multiplies the small elements of $G$ obtained by integrating $F$ over small squares inside $D$. It is not surprising that Driver and Sengupta had to use the coordinate system that allows to define a section through which $[A, A]=0$ in order to determine this order. The problem is that the class of loops along which they are able to define the random holonomy depends strongly on this choice of coordinates. 
It should be noted that B. Driver [Dr2] and L. Gross Gr] introduced a new local object in order to replace the white noise in this context and that this could lead to a way around the problem.

Although we do not treat this point in our work, we cannot conlude this conclusion without mentioning the semi-classical limit of the Yang-Mills measure. The remarkable fact is that, when the total surface of $M$ tends to 0 , the measure concentrates on the set of flat connections over $M$ and tends to the volume measure associated with the natural symplectic structure on this space. There are a lot of references on this subject which is closely related to the geometry of some moduli spaces [Fo, BS, KS, Se3, Liu, AB].

\section{Combinatorial approach.}

Our starting point is the combinatorial approach initiated by A.A. Migdal in 1975 [Mi] and improved by E.Witten in 1991 [Wi]. The idea is to replace the base space of the fiber bundle by a graph on a surface. This leads to a finite dimensional problem, where we define the random holonomy only along the paths of a graph. We also define conditional versions of the random holonomy. These conditional versions will play a technical role in the continuous construction on surfaces with boundary and lead also to the definition of very important objects, the conditional partition functions. The main property of the discrete theory is the invariance by subdivision. It explains that, up to some restrictions, the law of the random holonomy is independent of the graph in which one works.

The next step towards the continuum limit is to take the projective limit of the discrete measures associated with the graphs whose edges are piecewise geodesic for some Riemannian metric on $M$. This allows us to define a random holonomy along all piecewise geodesic paths on $M$. Then, we prove that this random holonomy can be extended by continuity to the set $P M$ of piecewise embedded paths on $M$, using a very natural approximation procedure. The law of this new random holonomy is a measure on $\mathcal{F}(P M, G)$ which is proved to be independent of all choices made during the construction. This measure is pushed forward on $\mathcal{F}(L M, G) / \mathcal{F}(M, G)$ and then becomes what we call the Yang-Mills measure. This measure is characterized by its consistence with the discrete theory and a continuity property. It is multiplicative, as a random holonomy is expected to be, and invariant by area-preserving diffeomorphisms.

All along the discrete construction, we study the special case $G=U(1)$. This analysis leads us to a second construction of a random holonomy, specific to the Abelian case, based on the Gaussian character of the measure in two dimensions. The characterization of the Yang-Mills measure given earlier allows us to prove that this random holonomy has the same law as that defined by the general procedure. Then we show that the holonomy along very small loops can be used to construct a white noise on $M$, by means of a Wiener-like integral.

It is then natural to try to adapt the extraction of the white noise to the general case. We prove a result in the semi-simple case that strongly suggests that this is impossible. Indeed, the $\sigma$-algebra generated by the holonomies along very small loops seems to satisfy a zero-one law.

In the last part of this work, we study combinatorial properties of the measure. We prove the Markov property of the random holonomy, extending to the continuous setting a result that was proved in the discrete setting by C. Becker and A. Sengupta BS. Then, we study how it is possible to glue together the Yang-Mills measures on two surfaces $M_{1}$ and $M_{2}$ in order to get the measure on $M$, the surface obtained by gluing $M_{1}$ and $M_{2}$ together. We show that the measures on $M_{1}$ and $M_{2}$ do not determine the measure on $M$. There is a lack of information 
that can be parametrized by the centralizer of the holonomy along the common boundary of $M_{1}$ and $M_{2}$. Finally, we summarize the algebraic properties of the conditional partition functions, whose importance had already been recognized by Witten [Wi]. We prove that a few of them generate all others by algebraic transformations and identify these elementary functions. We also show that the partition functions may be considered the transition functions of the random holonomy as a Markov field and discuss to what extent they determine the Yang-Mills measure. 


\section{Chapter 1}

\section{Discrete Yang-Mills measure}

In this chapter we construct and we study the discrete Yang-Mills measure. It is both the basis of the construction of the continuous Yang-Mills measure and the frame in which computations are possible. The main results are the invariance by subdivision of the discrete measure and the estimation of the law of the random holonomy along small loops.

\section{$1.1 \quad$ Notations}

Throughout this work, $M$ will denote a surface, i.e. a real differentiable two-dimensional manifold, compact, connected, orientable, with or without boundary. It is endowed with a Lebesguian measure $\sigma$, i.e. a measure which has positive smooth density with respect to the Lebesgue measure in any chart.

The boundary of $M$, if it is non empty, is the disjoint union of circles $N_{1}, \ldots, N_{p}$. Let us make explicit what we call smooth objects on $M$ and introduce the very useful notion of closure.

Definition 1.1.1 A closure of $M$ is a triple $\left(i, M, M_{1}\right)$, where $M_{1}$ is a closed surface, i.e. a surface without boundary and $i: M \longrightarrow M_{1}$ is an embedding. If the complementary of $i(M)$ in $M_{1}$ is diffeomorphic to a disjoint union of disks, the closure is said to be minimal.

Given two closures $\left(i_{1}, M, M_{1}\right)$ and $\left(i_{2}, M, M_{2}\right)$ of $M, i_{1}(M)$ and $i_{2}(M)$ have diffeomorphic neighbourhoods in $M_{1}$ and $M_{2}$. So it makes sense to say that an application (resp. a bundle, a section,...) is smooth on $M$ if it is the restriction of a smooth application (resp. bundle, section,...) defined on an open neighbourhood of $M$ in one of its closures.

The second basic object is $G$, a compact connected Lie group, that will be chosen to be Abelian or semi-simple in most examples.

Let $P$ be a principal $G$-bundle over $M$. If $M$ has a boundary, $M$ retracts on a bunch of circles and $P$ is trivial. But if $M$ is closed, the possible topological types for $P$ are classified by $\pi_{1}(G)$. A pleasant way to see this is to cut $M$ along the boundary of a small disk. We get two disjoint pieces. The restrictions of a bundle $P$ over $M$ to both pieces are trivial and the topology of $P$ is completely determined by the transition function along the boundary of the disk. This transition function is a map $S^{1} \longrightarrow G$ and it is a fact that two homotopic maps give rise to two homeomorphic bundles.

If $G=U(1)$, the element of $\pi_{1}(U(1)) \simeq \mathbf{Z}$ determined by $P$ corresponds to the Chern class of the complex line bundle associated with $P$. Note that when $G$ is semi-simple, $\pi_{1}(G)$ is finite. 
A connection $\omega$ on $P$ is a choice at each point $p$ of $P$ of a subspace in $T_{p} P$ supplementary to the vertical subspace of vectors tangent to the action of $G$. Moreover, this distribution, called the horizontal distribution, has to be invariant by the action of $G$.

Let $c:[0,1] \longrightarrow M$ be a regular path on $M$. A connection $\omega$ allows to lift $c$ to a horizontal path in $P$ starting at any prescribed point in $P_{c(0)}$. The function that maps a point $p$ of $P_{c(0)}$ to the end point of the horizontal lift of $c$ starting at $p$ is called the parallel transport or holonomy of $\omega$ along $c$. It is a $G$-equivariant map $\operatorname{hol}(\omega, c): P_{c(0)} \longrightarrow P_{c(1)}$. If $c_{1}$ and $c_{2}$ are two paths such that $c_{1}(1)=c_{2}(0)$, then the path $c_{1} c_{2}$ exists and we have

$$
\operatorname{hol}\left(\omega, c_{1} c_{2}\right)=\operatorname{hol}\left(\omega, c_{2}\right) \circ \operatorname{hol}\left(\omega, c_{1}\right)
$$

A gauge transformation is a diffeomorphism $j$ of $P$ over the identity of $M$ that commutes with the right action of $G$. Let $\omega$ be a connection on $P$. Let $c:[0,1] \longrightarrow M$ be a piecewise $C^{1}$ path. A gauge transformation $j$ allows to define a new connection $j^{*} \omega$ whose holonomy is related to that of $\omega$ through the relation:

$$
\operatorname{hol}\left(j^{*} \omega, c\right)=j(c(1))^{-1} \circ \operatorname{hol}(\omega, c) \circ j(c(0)) .
$$

Remark that these holonomies are conjugate if $c$ is a loop. More detailed presentations of the theory of fiber bundles and connections can be found for example in [KN, B]].

\subsection{Graphs on $M$}

In order to reduce to a discrete setting, we will replace $M$ by a graph drawn on $M$ and adapt the notions of fibre bundle, connection and gauge transformation.

\subsubsection{Pregraphs}

We say that an application $c:[0,1] \longrightarrow M$ is smooth (resp. an embedding) if it is the restriction of a smooth application (resp. embedding) defined on an open interval containing $[0,1]$.

Definition 1.2.1 A parametrized path on $M$ is a mapping $c:[0,1] \longrightarrow M$ which is the concatenation of a finite number of smooth embeddings.

Two parametrized paths are said to be equivalent if they differ by an increasing reparametrization.

Lemma 1.2.2 The equivalence of parametrized paths preserves their orientation, image, end points, injectivity, injectivity on $(0,1)$.

Equivalence classes of parametrized paths are called simply paths. The set of paths on $M$ is denoted by $P M$.

A path whose end points are equal is called a loop and a loop which is injective on $(0,1)$ is said to be simple. Given a path $a$, we denote by $a^{-1}$ the path obtained by reversing the orientation of $a$. An edge is an injective path $a$ such that $a([0,1]) \cap \partial M$ is empty or a finite union of segments. 
Definition 1.2.3 A pregraph on $M$ is a set $\Gamma=\left\{a_{1}, \ldots, a_{r}\right\}$ of edges that meet each other only at their end points, i.e. such that for each distinct $i$ and $j$ between 1 and $r$, one has

$$
a_{i}([0,1]) \cap a_{j}([0,1])=a_{i}(\{0,1\}) \cap a_{j}(\{0,1\})
$$

We call support of a pregraph $\Gamma$ the union of the images of its edges. A pregraph $\Gamma$ is said to be connected if its support $\operatorname{Supp}(\Gamma)$ is connected.

We call faces of a pregraph $\Gamma$ the connected components of $M \backslash \operatorname{Supp}(\Gamma)$. We denote by $\mathcal{F}(\Gamma)$ the set of these faces.

Proposition 1.2.4 Let $\Gamma$ be a connected pregraph on $M$. Suppose that every face of $\Gamma$ is diffeomorphic to a disk. Then the map $H_{1}(\operatorname{Supp}(\Gamma) ; \mathbf{Z}) \longrightarrow H_{1}(M ; \mathbf{Z})$ induced by the inclusion is surjective.

Proof. Let $c:[0,1] \longrightarrow M$ be a loop. There exists on each face of $\Gamma$ a point that is not in the image of $c$. Let us fix such a point in each face and remove it from $M$. The remaining open set $U$ retracts on the support of $\Gamma$ because each face with a point removed retracts on its boundary. This retraction induces a homotopy from $c$ to a loop whose image is included in $\operatorname{Supp}(\Gamma)$. So each loop of $M$ is homotopic, thus homologous to a loop of $\operatorname{Supp}(\Gamma)$. This proves the result.

\subsubsection{Graphs}

Given a pregraph $\Gamma$, we call path in $\Gamma$ a concatenation of edges of $\Gamma$, with natural or reverse orientation. We denote by $\Gamma^{*}$ the set of these paths.

Definition 1.2.5 A graph on $M$ is a connected pregraph $\Gamma$ whose faces are diffeomorphic to disks and such that for each component $N_{i}$ of $\partial M$, there exists an element of $\Gamma^{*}$ whose image is equal to $N_{i}$.

The reason for which we choose these properties is that we want a graph to take the whole topology of $M$ into account, including its boundary.

Definition 1.2.6 Let $\Gamma_{1}$ and $\Gamma_{2}$ be two pregraphs. We say that $\Gamma_{2}$ is finer than $\Gamma_{1}$ and write $\Gamma_{1}<\Gamma_{2}$ if each edge of $\Gamma_{1}$ is a path of $\Gamma_{2}^{*}$.

Proposition 1.2.7 Let $\Gamma_{0}$ be a pregraph on $M$. There exists a graph $\Gamma$ which is finer than $\Gamma_{0}$. Moreover, if $\operatorname{Supp}\left(\Gamma_{0}\right)$ is contained in an open set diffeomorphic to a disk, it is possible to construct $\Gamma$ in such a way that it has the same number of faces as $\Gamma_{0}$.

Lemma 1.2.8 A pregraph whose faces are diffeomorphic to disks is necessarily connected.

Proof. Let $\Gamma$ be a pregraph with faces diffeomorphic to disks. Suppose that $\Gamma=\Gamma^{\prime} \cup \Gamma^{\prime \prime}$, where $\Gamma^{\prime}$ and $\Gamma^{\prime \prime}$ have disjoint supports. Each face of $\Gamma$ is a disk, so it has a connected boundary, which is included either in the support of $\Gamma^{\prime}$ or in that of $\Gamma^{\prime \prime}$. The closures of the unions of faces whose boundary lies in $\operatorname{Supp}\left(\Gamma^{\prime}\right)\left(\operatorname{resp} . \operatorname{Supp}\left(\Gamma^{\prime \prime}\right)\right)$ form a partition of $M$ into two closed sets, 
which is in contradiction with connectedness of $M$.

Before to prove proposition 1.2.7, let us recall some classical facts about the topology of $M$. If $M$ has no boundary and is not a sphere, its universal covering is diffeomorphic to a plane. In this plane, it is always possible to choose a polygonal fundamental domain for the covering map, namely a $4 g$-gonal domain if $g$ is the genus of $M$. This means that it is possible to see topologically $M$ as the result of the identification of some edges of a polygon. If $M$ is a shpere, it can be seen as a disk whose upper and lower half of the boundary have been identified. If $M$ has a boundary, there are holes in the universal covering, one for each boundary component in a fundamental domain. It is possible to choose a fundamental domain such that the holes are in its interior. Thus, it is possible to represent a surface with boundary by a picture like picture 1.1.
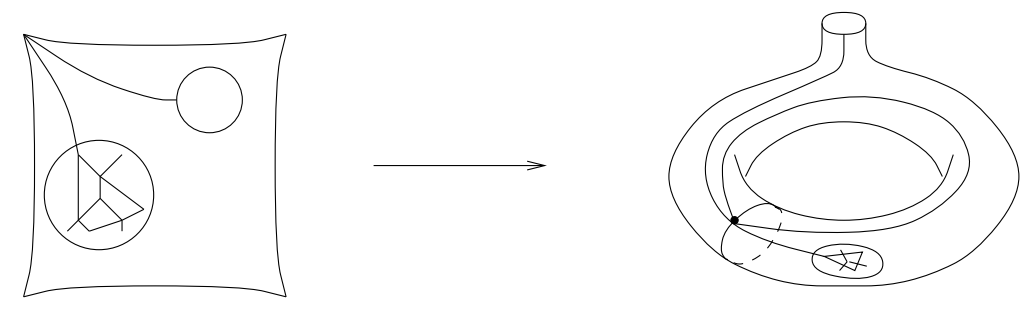

Figure 1.1: Fundamental domain in the universal covering for a torus with one hole.

Proofof proposition 1.2.7. By definition of an edge, the set $\operatorname{Supp}\left(\Gamma_{0}\right) \cap \partial M$ is a finite union of segments. Cutting some edges of $\Gamma_{0}$ in several pieces if necessary, we can assume that these segments are exactly images of edges. Then, it is possible to add to $\Gamma_{0}$ edges in such a way that $\operatorname{Supp}\left(\Gamma_{0}\right) \cap \partial M=\partial M$. If $\operatorname{Supp}\left(\Gamma_{0}\right)$ did not meet a component of $\partial M$ initially, it is necessary to add at least two edges on this component. So we can construct a pregraph $\Gamma_{1}$ which is finer than $\Gamma_{0}$, whose support contains $\partial M$.

Each face of $\Gamma_{1}$ is homeomorphic to the interior of a compact surface with boundary. On any such surface, there exists a graph, for example a triangulation. We add to $\Gamma_{1}$ the edges that are necessary to transform it into a graph on each face which is not diffeomorphic to a disk. All faces of the resulting pregraph $\Gamma_{2}$ are diffeomorphic to disks. By the preceding lemma, it is connected. Thus, it is a graph. The first part of the proposition is proved.

If $\operatorname{Supp}\left(\Gamma_{0}\right)$ is contained in a disk, it is possible to move this disk by a diffeomorphism of $M$ into any prescribed disk. It is possible to get the situation described by the picture, in the universal covering, where the disk is in the interior of a fundamental domain. Then it is easy to complete $\Gamma_{0}$ into a graph with the same number of faces and get back to the initial situation by the inverse diffeomorphism.

\subsection{Discrete holonomy and gauge transformations}

Choose a graph $\Gamma=\left\{a_{1}, \ldots, a_{r}\right\}$ on $M$ and denote by $i: \operatorname{Supp}(\Gamma) \longrightarrow M$ the canonical injection. Consider the fiber bundle $i^{*} P$ on $\operatorname{Supp}(\Gamma)$. It is a trivial bundle, whatever the topology of $P$ was. We identify $i^{*} P$ with $\operatorname{Supp}(\Gamma) \times G$. Let $\mathcal{V}(\Gamma)$ denote the set of vertices of $\Gamma$. 
Lemma 1.3.1 Let $\omega_{1}$ and $\omega_{2}$ be two connections on $i^{*} P$. Suppose that $\omega_{1}$ and $\omega_{2}$ have the same holonomy along each edge of $\Gamma$. Then there exists a gauge transformation $j$ of $i^{*} P$ that leaves the fibers over the points of $\mathcal{V}(\Gamma)$ invariant and such that $j^{*} \omega_{1}=\omega_{2}$.

Proof. Choose a parametrization of $a_{1} \in \Gamma$. Set

$$
j\left(a_{1}(t)\right)=\operatorname{hol}\left(\omega_{1}, a_{1 \mid[0, t]}\right) \circ \operatorname{hol}\left(\omega_{2},\left(a_{1 \mid[0, t]}\right)^{-1}\right) .
$$

Then

$$
\operatorname{hol}\left(\omega_{2}, a_{1 \mid[0, t]}\right)=j\left(a_{1}(t)\right)^{-1} \circ \operatorname{hol}\left(\omega_{1}, a_{1 \mid[0, t]}\right)=\operatorname{hol}\left(j^{*} \omega_{1}, a_{1 \mid[0, t]}\right) .
$$

The assumption about $\omega_{1}$ and $\omega_{2}$ makes sure that it is possible to extend the construction of $j$ to the whole graph and that $j(m)=\operatorname{Id}_{P_{m}}$ for all vertex $m$ of $\Gamma$.

This implies that $\omega_{2}$ and $j^{*} \omega_{1}$ have the same holonomy, hence the same horizontal paths, so they are equal.

In the discrete setting, we expect to be able to compute the holonomy only along edges of the graph. So we identify a connection with the holonomy that it determines along the edges of $\Gamma$ and, according to the preceding lemma, consider gauge transformations that act only on the fibers over the vertices of $\Gamma$. Finally, using the identification $i^{*} P=\operatorname{Supp}(\Gamma) \times G$, we can identify holonomies and gauge transformations with elements of $G$, defining for example $h_{c}(\omega)$ and $j(m)$ by $\operatorname{hol}(\omega, c)(c(0), 1)=\left(c(1), h_{c}(\omega)\right)$ and $j(m, 1)=(m, j(m))$.

This leads us to the following definitions.

Definition 1.3.2 A discrete connection on $\Gamma$ is a map from $\Gamma$ into $G$. A discrete gauge transformation is a map from the set $\mathcal{V}(\Gamma)$ of vertices of $\Gamma$ into $G$.

A discrete gauge transformation $j: \mathcal{V}(\Gamma) \longrightarrow G$ acts on a discrete connection $g=\left(g_{1}, \ldots, g_{r}\right)$ by :

$$
j \cdot g=\left(j\left(a_{1}(1)\right)^{-1} g_{1} j\left(a_{1}(0)\right), \ldots, j\left(a_{r}(1)\right)^{-1} g_{r} j\left(a_{r}(0)\right)\right) .
$$

A discrete connection $g=\left(g_{1}, \ldots, g_{r}\right)$ determines a multiplicative application from $\Gamma^{*}$ into $G$. Given a path $c=a_{i_{1}}^{\varepsilon_{1}} \ldots a_{i_{n}}^{\varepsilon_{n}}, \varepsilon_{i}= \pm 1$, we can compute $g_{i_{n}}^{\varepsilon_{n}} \ldots g_{i_{1}}^{\varepsilon_{1}}$ (with reversed order!). In other words, any path $c$ of $\Gamma^{*}$ gives rise to a map $h_{c}$ from $G^{\Gamma}$ to $G$ defined by

$$
h_{c}\left(g_{1}, \ldots, g_{r}\right)=g_{i_{n}}^{\varepsilon_{n}} \ldots g_{i_{1}}^{\varepsilon_{1}} .
$$

This map is well defined because there is only one way to decompose a path in product of edges. To see why, it is enough to consider the times at which a path crosses a vertex of $\Gamma$.

Proposition 1.3.3 Let $c_{1}$ and $c_{2}$ be two paths such that $c_{1} c_{2}$ is also a path. Then

$$
h_{c_{1} c_{2}}=h_{c_{2}} h_{c_{1}} .
$$

This basic property of the discrete holonomy will be refered to as the multiplicativity of the holonomy.

A gauge transformation $j$ transforms $h_{c}$ in $h_{c} \circ j$, with

$$
h_{c} \circ j=j(c(1))^{-1} h_{c} j(c(0)) .
$$

At this stage, it may be noticed that central functions of the holonomy along loops are invariant under gauge transformations. This is why they will play such a major role in the sequel. 


\subsection{Discrete Yang-Mills measure}

We keep a graph $\Gamma=\left\{a_{1}, \ldots, a_{r}\right\}$ on $M$. In the discrete setting, a probability measure on the quotient space $\mathcal{A} / \mathcal{J}$ of connections modulo gauge transformations is represented by a probability measure on $G^{\Gamma}$ invariant under the action of $G^{\mathcal{V}(\Gamma)}$.

The basic example of such an invariant measure is the product of Haar measures. We shall construct the discrete Yang-Mills on $G^{\Gamma}$ as :

$$
d P=\frac{d P}{d g} d g
$$

where $d g=d g_{1} \otimes \ldots \otimes d g_{r}$. The density $\frac{d P}{d g}$ will be a product of central functions of holonomies along loops, a feature that makes $P$ invariant. Recall that a function $p$ is said to be central on $G$ if $p(x y)=p(y x)$, or equivalently $p\left(y^{-1} x y\right)=p(x)$ for all $x, y$ in $G$.

Each face $F$ of $\Gamma$ has a boundary which is the image of a path defined up to the choice of an origin and an orientation. So the function $h_{\partial F}: G^{\Gamma} \longrightarrow G$ is defined up to conjugation and inversion. For any central function $p$ invariant by inversion, the function $p \circ h_{\partial F}$ is well defined.

Let us denote by $\left(p_{t}\right)_{t>0}$ the fundamental solution of the heat equation on $G$ endowed with its biinvariant Riemannian metric, normalized to have total volume equal to 1 . It satisfies

$$
\left(\partial_{t}-\frac{1}{2} \Delta\right) p_{t}=0 \quad \text { on } \quad \mathbf{R}_{+}^{*} \times G,
$$

and for any function $f$ continuous on $G$,

$$
\int_{G} f(g) p_{t}(g) d g \underset{t \rightarrow 0}{\longrightarrow} f(1)
$$

For any positive $t, p_{t}$ is a positive central function, invariant by inversion, such that $\int_{G} p_{t}(g) d g=$ 1. For the moment, the choice of the heat kernel may seem to be quite arbitrary. We shall discuss this point at the end of section 1.6 .

For each face $F$ of $\Gamma$, the function $p_{\sigma(F)}\left(h_{\partial F}\right): G^{\Gamma} \longrightarrow \mathbf{R}_{+}^{*}$ is well defined, with $\sigma$ denoting the surface measure on $M$. Set

$$
\begin{gathered}
D=\prod_{F \in \mathcal{F}(\Gamma)} p_{\sigma(F)}\left(h_{\partial F}\right): G^{\Gamma} \longrightarrow \mathbf{R}_{+}^{*}, \\
Z=\int_{G^{\Gamma}} D d g .
\end{gathered}
$$

Now define $P$ on $\left(G^{\Gamma}, \operatorname{Bor}\left(G^{\Gamma}\right)\right)$ by

$$
d P=\frac{1}{Z} D d g
$$

Given $n$ paths $c_{1}, \ldots, c_{n}$ in $\Gamma^{*}$, we define the law of the discrete holonomy along $c_{1}, \ldots, c_{n}$ as the joint law of the $n$-uple $\left(h_{c_{1}}, \ldots, h_{c_{n}}\right)$ under $P$.

\subsection{Conditional Yang-Mills measure}

When $M$ has a boundary, it is natural to want to impose the holonomy along the components of $\partial M$. It may also be useful to be able to impose holonomy along some other loops even if $M$ has no boundary. 


\subsubsection{Conditional Haar measure}

Proposition 1.5.1 Let $n$ be a positive integer. Let $x$ be an element of $G$. There exists on $G^{n}$ a measure $\nu_{x}^{n}$ such that $g_{n} \ldots g_{1}=x \nu_{x}^{n}$-a.s. and such that for any function $f$ continuous on $G^{n}$ and any $i$ between 1 and $n$,

$$
\begin{array}{r}
\nu_{x}^{n}(f)=\int_{G^{n-1}} f\left(g_{1}, \ldots, g_{i-1},\left(g_{n} \ldots g_{i+1}\right)^{-1} x\left(g_{i-1} \ldots g_{1}\right)^{-1}, g_{i+1}, \ldots, g_{n}\right) \\
d g_{1} \ldots \widehat{d g_{i}} \ldots d g_{n} .
\end{array}
$$

Moreover, one has

$$
\nu_{x}^{n}(f)=\lim _{t \rightarrow 0} \int_{G^{n}} f\left(g_{1}, \ldots, g_{n}\right) p_{t}\left(g_{n} \ldots g_{1} x^{-1}\right) d g_{1} \ldots d g_{n} .
$$

Finally, $\int_{G} \nu_{x}^{n} d x=d g$, as measures on $G^{n}$.

Proof. Pick $i$ between 1 and $n$, and $t>0$. By centrality of $p_{t}$ and then right invariance of $d g_{i}$, one has

$$
\begin{aligned}
& \int_{G^{n}} f\left(g_{1}, \ldots, g_{n}\right) p_{t}\left(g_{n} \ldots g_{1} x^{-1}\right) d g= \\
= & \int_{G^{n}} f\left(g_{1}, \ldots, g_{n}\right) p_{t}\left(g_{i}\left(g_{i-1} \ldots g_{1}\right) x^{-1}\left(g_{n} \ldots g_{i+1}\right)\right) d g \\
= & \int_{G^{n}} f\left(g_{1}, \ldots, g_{i-1}, g_{i}\left(g_{n} \ldots g_{i+1}\right)^{-1} x\left(g_{i-1} \ldots g_{1}\right)^{-1}, g_{i+1}, \ldots, g_{n}\right) p_{t}\left(g_{i}\right) \\
\underset{t \rightarrow 0}{\longrightarrow} & \int_{G^{n-1}} f\left(g_{1}, \ldots, g_{i-1},\left(g_{n} \ldots g_{i+1}\right)^{-1} x\left(g_{i-1} \ldots g_{1}\right)^{-1}, g_{i+1}, \ldots, g_{n}\right) \\
& d g_{1} \ldots \widehat{d g_{i}} \ldots d g_{n}
\end{aligned}
$$

Thus the limit exists and the last expression does not depend on $i$. It defines a probability measure on $G^{n}$.

If $f$ vanishes on the hypersurface $\left\{g_{1} \ldots g_{n}=x\right\}$, then $\nu_{x}^{n}(f)=0$. So we do have $g_{n} \ldots g_{1}=x$ $\nu_{x}^{n}$-a.s.

Finally, since $\int_{G} p_{t}(g) d g=1$,

$$
\int_{G} \int_{G^{n}} f\left(g_{1}, \ldots, g_{n}\right) p_{t}\left(g_{n} \ldots g_{1} x^{-1}\right) d x d g=\int_{G^{n}} f\left(g_{1}, \ldots, g_{n}\right) d g,
$$

which implies the last statement when $t$ tends to zero.

\subsubsection{Conditional Yang-Mills measure}

Let $L_{1}, \ldots, L_{q}$ be disjoint simple loops of $\Gamma^{*}$ whose image is either a component of $\partial M$ or contained in the interior of $M$. We want to choose the law of $\left(h_{L_{1}}, \ldots, h_{L_{q}}\right)$. For this, it is enough to be able to impose a deterministic value to each $h_{L_{i}}$. Let $\left(x_{1}, \ldots, x_{q}\right)$ be an element of $G^{q}$. Let $\Gamma^{\prime}$ denote the set of edges of $\Gamma$ that do not appear in the decomposition of any $L_{i}$. We denote by $d g^{\prime}$ the product of Haar measures on $G^{\Gamma^{\prime}}$. The fact that the conditional Haar 
measure is not invariant by permutation of the factors on $G^{n}$ leads either to a very heavy or to an elliptic notation. We will choose the second option, except during a few lines. Suppose that $L_{1}=a_{i_{1}^{1}}^{\varepsilon_{1}^{1}} \ldots a_{i_{n_{1}}^{1}}^{\varepsilon_{n_{1}}^{1}}, \ldots, L_{q}=a_{i_{1}^{q}}^{\varepsilon_{1}^{q}} \ldots a_{i_{n_{q}}^{q}}^{\varepsilon_{q}^{q}}$, with $\varepsilon_{j}^{i}= \pm 1$. We denote by $d \nu_{x_{1}} \ldots d \nu_{x_{q}} d g^{\prime}$ the following measure on $G^{\Gamma}$ :

$$
d \nu_{x_{1}}^{n_{1}}\left(g_{i_{1}^{1}}^{\varepsilon_{1}^{1}}, \ldots, g_{i_{n_{1}}^{1}}^{\varepsilon_{n_{1}}^{1}}\right) \ldots d \nu_{x_{q}}^{n_{q}}\left(g_{i_{1}^{q}}^{\varepsilon_{1}^{q}}, \ldots, g_{i_{n_{q}}^{q}}^{\varepsilon_{q}^{q}}\right) d g^{\prime}
$$

With this notation, set:

$$
\begin{gathered}
Z\left(x_{1}, \ldots, x_{q}\right)=\int_{G^{\Gamma}} D d \nu_{x_{1}} \ldots d \nu_{x_{q}} d g^{\prime} \\
d P\left(x_{1}, \ldots, x_{q}\right)=\frac{1}{Z\left(x_{1}, \ldots, x_{q}\right)} D d \nu_{x_{1}} \ldots d \nu_{x_{q}} d g^{\prime} .
\end{gathered}
$$

The function $Z\left(x_{1}, \ldots, x_{q}\right)$ is called conditional partition function on $M$ with respect to $L_{1}, \ldots, L_{q}$.

Proposition 1.5.2 Choose $r \leq q$ and $x_{r+1}, \ldots, x_{q} \in G$. The distribution of $\left(h_{L_{1}}, \ldots, h_{L_{r}}\right)$ under $P\left(x_{r+1}, \ldots, x_{q}\right)$ is equal to:

$$
\frac{Z\left(x_{1}, \ldots, x_{q}\right)}{Z\left(x_{r+1}, \ldots, x_{q}\right)} d x_{1} \ldots d x_{r}
$$

where each element $x_{i}$ corresponds to the loop $L_{i}$. In particular, the distribution of $\left(h_{L_{1}}, \ldots, h_{L_{q}}\right)$ under $P$ is $\frac{1}{Z} Z\left(x_{1}, \ldots, x_{q}\right) d x_{1} \ldots d x_{q}$.

Proof: The last part of the statement is just the case $r=q$. Let $f$ be a continuous function on $G^{r}$. We have:

$$
\begin{aligned}
\int_{G^{\Gamma}} & f\left(h_{L_{1}}, \ldots, h_{L_{r}}\right) d P\left(x_{r+1}, \ldots, x_{q}\right)= \\
= & \frac{1}{Z\left(x_{r+1}, \ldots, x_{q}\right)} \int_{G^{\Gamma}} f\left(h_{L_{1}}, \ldots, h_{L_{r}}\right) D d \nu_{x_{r+1}} \ldots d \nu_{x_{q}} d g^{\prime} \\
= & \frac{1}{Z\left(x_{r+1}, \ldots, x_{q}\right)} \int_{G^{r}} \int_{G^{\Gamma}} f\left(h_{L_{1}}, \ldots, h_{L_{r}}\right) D d \nu_{x_{1}} \ldots d \nu_{x_{q}} d g^{\prime} d x_{1} \ldots d x_{r} \\
= & \int_{G^{r}} f\left(x_{1}, \ldots, x_{r}\right)\left[\frac{1}{Z\left(x_{r+1}, \ldots, x_{q}\right)} \int_{G^{\Gamma}} D d \nu_{x_{1}} \ldots d \nu_{x_{q}} d g^{\prime}\right] d x_{1} \ldots d x_{r} \\
= & \int_{G^{r}} f\left(x_{1}, \ldots, x_{r}\right) \frac{Z\left(x_{1}, \ldots, x_{q}\right)}{Z\left(x_{r+1}, \ldots, x_{q}\right)} d x_{1} \ldots d x_{r} .
\end{aligned}
$$

Corollary 1.5.3 The map $\left(x_{1}, \ldots, x_{q}\right) \mapsto P\left(x_{1}, \ldots, x_{q}\right)$ is a disintegration of the measure $P$ with respect to the random variable $\left(h_{L_{1}}, \ldots, h_{L_{q}}\right)$. This means that

1. $\left(h_{L_{1}}, \ldots, h_{L_{q}}\right)=\left(x_{1}, \ldots, x_{q}\right) P\left(x_{1}, \ldots, x_{q}\right)$-a.s.

2. denoting by $\eta$ the law of $\left(h_{L_{1}}, \ldots, h_{L_{q}}\right)$ under $P$, we have

$$
P=\int_{G^{q}} P\left(x_{1}, \ldots, x_{q}\right) d \eta\left(x_{1}, \ldots, x_{q}\right) .
$$


Proof. The first part is a direct consequence of the definition of $P\left(x_{1}, \ldots, x_{q}\right)$. A simple computation proves the second one:

$$
\begin{aligned}
\int_{G^{q}} P\left(x_{1}, \ldots, x_{q}\right) d \eta\left(x_{1}, \ldots, x_{q}\right) & =\frac{1}{Z} \int_{G^{q}} P\left(x_{1}, \ldots, x_{q}\right) Z\left(x_{1}, \ldots, x_{q}\right) d x_{1} \ldots d x_{q} \\
& =\frac{1}{Z} \int_{G^{q}} D \nu_{x_{1}} \ldots \nu_{x_{q}} d g^{\prime} d x_{1} \ldots d x_{q} \\
& =P .
\end{aligned}
$$

This corollary says that the $P\left(x_{1}, \ldots, x_{q}\right)$ are really what we expected them to be. Now, given a measure $\beta$ on $G^{q}$, we can choose the law of $\left(h_{L_{1}}, \ldots, h_{L_{q}}\right)$ to be $\beta$ by putting the measure $P_{\beta}=\int_{G^{q}} P\left(x_{1}, \ldots, x_{q}\right) d \beta\left(x_{1}, \ldots, x_{q}\right)$ on $G^{\Gamma}$.

\subsubsection{Gauge transformations}

Let us compute how the conditional measure $P\left(x_{1}, \ldots, x_{q}\right)$ is transformed by a gauge transformation.

Lemma 1.5.4 Let $j$ be a discrete gauge transformation. The following equality holds:

$$
j_{*} P\left(x_{1}, \ldots, x_{q}\right)=P\left(y_{1} x_{1} y_{1}^{-1}, \ldots, y_{q} x_{q} y_{q}^{-1}\right),
$$

where $y_{i}=j\left(L_{i}(0)\right)$.

Proof. For sake of simplicity, let us write the proof in the case $q=1$, the general case being exactly similar, only with heavier notations. Suppose that $L_{1}=a_{1} \ldots a_{m}$. Let $f$ be a continuous function on $G^{\Gamma}$.

$$
\begin{aligned}
j_{*} P\left(x_{1}\right)(f) & =\frac{1}{Z\left(x_{1}\right)} \int_{G^{\Gamma}} f \circ j \prod_{F \in \mathcal{F}(\Gamma)} p_{\sigma(F)}\left(h_{\partial F}\right) \nu_{x_{1}}^{m}\left(g_{1}, \ldots, g_{m}\right) d g_{m+1} \ldots d g_{r} \\
& =\frac{1}{Z\left(x_{1}\right)} \int_{G^{\Gamma}} f \prod_{F \in \mathcal{F}(\Gamma)} p_{\sigma(F)}\left(h_{\partial F} \circ j^{-1}\right) \nu_{x_{1}}^{m}\left(j\left(a_{1}(1)\right) g_{1} j\left(a_{1}(0)\right)^{-1}, \ldots\right. \\
& =\frac{1}{Z\left(x_{1}\right)} \int_{G^{\Gamma}} f \prod_{F \in \mathcal{F}(\Gamma)} p_{\sigma(F)}\left(h_{\partial F}\right) \nu_{j\left(L_{1}(0)\right) x_{1} j\left(L_{1}(0)\right)^{-1}}^{m}\left(g_{1}, \ldots, g_{m}\right) \\
& d g_{m+1} \ldots d g_{r} \\
& =\frac{Z\left(y_{1} x_{1} y_{1}^{-1}\right)}{Z\left(x_{1}\right)} P\left(y_{1} x_{1} y_{1}^{-1}\right)(f),
\end{aligned}
$$

with $y_{1}=j\left(L_{1}(0)\right)$. Setting $f$ to be identically equal to 1 , we get $Z\left(y_{1} x_{1} y_{1}^{-1}\right)=Z\left(x_{1}\right)$.

Let us state the invariance property of the partition function that we just proved:

Proposition 1.5.5 For any $y_{1}, \ldots, y_{q}$ in $G$, one has

$$
Z\left(y_{1}^{-1} x_{1} y_{1}, \ldots, y_{q}^{-1} x_{q} y_{q}\right)=Z\left(x_{1}, \ldots, x_{q}\right) .
$$


According to this result, the conditional partition function can also be viewed as a function on $(G / \mathrm{Ad})^{q}$, where $A d$ is the adjoint action on $G$ given by $\operatorname{Ad}(y) x=y^{-1} x y$. We will use this point of view in the next paragraph.

It is clear now that $P\left(x_{1}, \ldots, x_{q}\right)$ is not gauge invariant in general. We will explain how to overcome this problem.

Let $t$ be an element of the quotient $G / \mathrm{Ad}$, that is, a conjugacy class in $G$. Let $x$ be an element of this class. The measure $\int_{G} \delta_{y x y^{-1}} d y$ does not depend on the choice of $x$ in $t$. We shall denote it by $\delta_{t}$. Similarly, we denote $\delta_{t_{1}} \otimes \ldots \otimes \delta_{t_{q}}$ by $\delta_{t_{1}, \ldots, t_{q}}$. Set

$$
P\left(t_{1}, \ldots, t_{q}\right)=\int_{G^{q}} P\left(x_{1}, \ldots, x_{q}\right) \delta_{t_{1}, \ldots, t_{q}}\left(x_{1}, \ldots, x_{q}\right) .
$$

Proposition 1.5.6 The measure $P\left(t_{1}, \ldots, t_{q}\right)$ is gauge invariant.

Proof: Let $j$ be a discrete gauge transformation and set $y_{i}=j\left(L_{i}(0)\right)$. According to the lemma 1.5.4, we have:

$$
\begin{aligned}
j_{*} P\left(t_{1}, \ldots, t_{q}\right) & =\int_{G^{q}} j_{*} P\left(x_{1}, \ldots, x_{q}\right) \delta_{t_{1}, \ldots, t_{q}}\left(x_{1}, \ldots, x_{q}\right) \\
& =\int_{G^{q}} P\left(y_{1} x_{1} y_{1}^{-1}, \ldots, y_{q} x_{q} y_{q}^{-1}\right) \delta_{t_{1}, \ldots, t_{q}}\left(x_{1}, \ldots, x_{q}\right) \\
& =\int_{G^{q}} P\left(y_{1} z_{1} x_{1}^{0} z_{1}^{-1} y_{1}^{-1}, \ldots, y_{q} z_{q} x_{q}^{0} z_{q}^{-1} y_{q}^{-1}\right) d z_{1} \ldots d z_{q} \\
& =\int_{G^{q}} P\left(z_{1}^{-1} x_{1}^{0} z_{1}, \ldots, z_{q}^{-1} x_{q}^{0} z_{q}\right) d z_{1} \ldots d z_{q} \\
& =\int_{G^{q}} P\left(x_{1}, \ldots, x_{q}\right) \delta_{t_{1}, \ldots, t_{q}}\left(x_{1}, \ldots, x_{q}\right) \\
& =P\left(t_{1}, \ldots, t_{q}\right),
\end{aligned}
$$

where each $x_{i}^{0}$ was an arbitrary element of $t_{i}$.

It will emerge later that the measures $P\left(t_{1}, \ldots, t_{q}\right)$ are in fact more natural than the $P\left(x_{1}, \ldots, x_{q}\right)$.

\subsection{Invariance by subdivision}

The invariance by subdivision is the main feature of the discrete theory. It allows to prove that the law of the discrete holonomy along given loops does not depend on the graph in which one computes it.

The fact that the heat kernel $\left(p_{t}\right)_{t>0}$ is a convolution semi-group will play a central role in the proof. This means that for any $x \in G$ and any $s, t$ such that $0<s<t$,

$$
\int_{G} p_{s}\left(x y^{-1}\right) p_{t}(y) d y=p_{t}(x) \text {. }
$$

Let $\Gamma_{1}$ and $\Gamma_{2}$ be two graphs on $M$. Suppose that $\Gamma_{2}$ is finer than $\Gamma_{1}$ and set $\Gamma_{1}=$ $\left\{a_{1} \ldots, a_{r}\right\}$. By definition, each edge $a_{i}$ of $\Gamma_{1}$ is a path in $\Gamma_{2}^{*}$ and it gives rise to a function $h_{a_{i}}: G^{\Gamma_{2}} \longrightarrow G$. The $r$-uple of those functions constitutes a single function $\left(h_{a_{1}}, \ldots, h_{a_{r}}\right)$ : $G^{\Gamma_{2}} \longrightarrow G^{r}=G^{\Gamma_{1}}$ that we denote by $f_{\Gamma_{1} \Gamma_{2}}$. the invariance by subdivision is expressed by the following result : 
Theorem 1.6.1 Let $\Gamma_{1}$ and $\Gamma_{2}$ be two graphs on $M$ such that $\Gamma_{2}$ is finer than $\Gamma_{1}$. Let $L_{1}, \ldots, L_{q}$ be disjoint simple loops of $\Gamma_{1}^{*}$. Let $x_{1}, \ldots, x_{q}$ be elements of $G$. Then

1. The map $f_{\Gamma_{1} \Gamma_{2}}: G^{\Gamma_{2}} \longrightarrow G^{\Gamma_{1}}$ is surjective.

2. This map satisfies : $\left(f_{\Gamma_{1} \Gamma_{2}}\right)_{*} P^{\Gamma_{2}}\left(x_{1}, \ldots, x_{q}\right)=P^{\Gamma_{1}}\left(x_{1}, \ldots, x_{q}\right)$.

From now on, it will be sometimes necessary to write explicitely the graph in which we consider objects such as $P, Z, D$.

We begin by proving that it is always possible to go from one graph to a finer graph by a finite sequence of elementary transformations.

Lemma 1.6.2 Let $\Gamma$ and $\Gamma^{\prime}$ be two graphs such that $\Gamma<\Gamma^{\prime}$. There exist an increasing sequence of graphs $\Gamma_{0}=\Gamma<\Gamma_{1}<\ldots<\Gamma_{n}<\ldots$, stationnary of limit $\Gamma^{\prime}$ and such that for any nonnegative $n$, one can transform $\Gamma_{n}$ into $\Gamma_{n+1}$ by one of the two following elementary operations:

(V) Add a vertex to $\Gamma_{n}$, i.e. replace an edge a by two edges $b$ and $c$ such that $a=b c$,

(E) Add an edge to $\Gamma_{n}$, this new edge joining two vertices of $\Gamma_{n}$.

Proof. We proceed by induction on $n$. $\Gamma_{0}$ is given, equal to $\Gamma$. Suppose $\Gamma_{n}$ given, with $\Gamma_{n}<\Gamma^{\prime}$. Recall that $\mathcal{V}(\Gamma)$ denotes the set of vertices of $\Gamma$.

- We have $\mathcal{V}\left(\Gamma_{n}\right) \subset \mathcal{V}\left(\Gamma^{\prime}\right) \cap \operatorname{Supp}\left(\Gamma_{n}\right)$. If this inclusion is a strict one, pick an element of $\left(\mathcal{V}\left(\Gamma^{\prime}\right) \cap \operatorname{Supp}\left(\Gamma_{n}\right)\right) \backslash \mathcal{V}\left(\Gamma_{n}\right)$. It is a vertex of $\Gamma^{\prime}$ which is on an edge of $\Gamma_{n}$ whithout being one of its end points. By an operation $(\mathrm{V})$, we add this vertex to $\Gamma_{n}$ and get $\Gamma_{n+1}$ which is still finer than $\Gamma^{\prime}$. Note that $\operatorname{Card}\left(\Gamma_{n+1}\right)=\operatorname{Card}\left(\Gamma_{n}\right)+1$.

- If $\mathcal{V}\left(\Gamma_{n}\right)=\mathcal{V}\left(\Gamma^{\prime}\right) \cap \operatorname{Supp}\left(\Gamma_{n}\right)$, then each edge of $\Gamma_{n}$ is an edge of $\Gamma^{\prime}$. In other words, $\Gamma_{n} \subset \Gamma^{\prime}$. If this inclusion is a strict one, there exists an edge of $\Gamma^{\prime}$ which is not an edge of $\Gamma_{n}$ and by connectedness of $\Gamma^{\prime}$ we may assume that this edge has at least one of its end points on $\operatorname{Supp}\left(\Gamma_{n}\right)$. By an operation (E), we add this edge to $\Gamma_{n}$ and get $\Gamma_{n+1}$ which is still connected and finer than $\Gamma^{\prime}$. The pregraph $\Gamma_{n+1}$ is a graph. Indeed, we just noticed that it is connected and it is finer than $\Gamma_{0}$, so that its support contains $\partial M$. It can happen that the operation (E) cuts a face in two pieces, but they are still diffeomorphic to disks. We also have $\operatorname{Card}\left(\Gamma_{n+1}\right)=\operatorname{Card}\left(\Gamma_{n}\right)+1$. - If $\Gamma_{n}=\Gamma^{\prime}$, just set $\Gamma_{n+1}=\Gamma_{n}$.

At each step, the fact that $\Gamma_{n}$ is a graph implies that $\Gamma_{n+1}$ is also a graph : connectedness is preserved, as well as boundary properties. The faces of a graph are not modified by an operation (V) and it can happen that an operation (E) cuts a face into two pieces, which are still diffeomorphic to disks.

For each $n, \Gamma_{n}<\Gamma^{\prime}$ implies $\operatorname{Card}\left(\Gamma_{n}\right) \leq \operatorname{Card}\left(\Gamma^{\prime}\right)$. On the other hand, elementary operations increase strictly the cardinal of the graph. Thus, there is necessarily only a finite number of such operations before the sequence becomes stationnary.

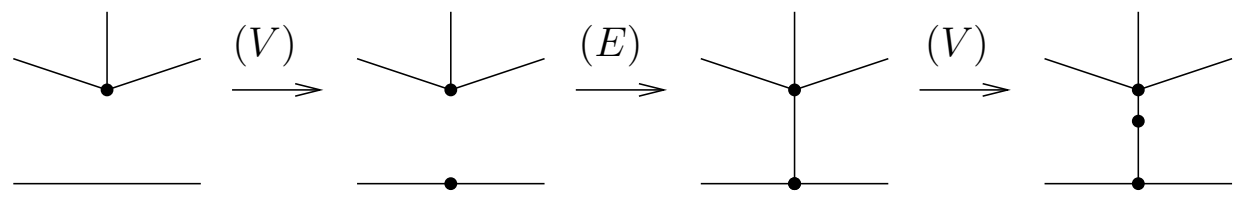

Figure 1.2: Examples of elementary transformations of a graph. 
Lemma 1.6.3 Let $\Gamma_{1}<\Gamma_{2}<\Gamma_{3}$ be three graphs. Then

$$
f_{\Gamma_{1} \Gamma_{3}}=f_{\Gamma_{1} \Gamma_{2}} \circ f_{\Gamma_{2} \Gamma_{3}} .
$$

Proof. It is the associativity of the product in $G$.

This lemma shows that it is enough to prove the theorem 1.6.1 when $\Gamma_{2}$ can be deduced from $\Gamma_{1}$ by an elementary operation. One recovers the general case by composition.

During the proof, we set $\beta=\delta_{\left(x_{1}, \ldots, x_{q}\right)}$ and use the notations $P_{\beta}=P\left(x_{1}, \ldots, x_{q}\right)$ and $Z_{\beta}=Z\left(x_{1}, \ldots, x_{q}\right)$ in order to make the expressions shorter.

Proof of theorem 1.6.1 : 1 . We prove that $f_{\Gamma_{1} \Gamma_{2}}$ is surjective. If $\Gamma_{2}$ can be deduced from $\Gamma_{1}$ by an operation (E), $f_{\Gamma_{1} \Gamma_{2}}$ is just the projection that forgets the factor associated with the new edge. It is of course surjective. In the case of an operation $(\mathrm{V}), f_{\Gamma_{1} \Gamma_{2}}$ preserves all factors except those associated with the two new edges, that are multiplied. It is also surjective.

2. Let us begin by the case of an operation of type (E). We fix some notations. Set $\Gamma_{1}=$ $\left\{a_{1}, \ldots, a_{r}\right\}$ and $\Gamma_{2}=\left\{a_{1}, \ldots, a_{r}, b\right\}$. The new edge $b$ is located in a face $F_{0}$ of $\Gamma_{1}$. Two situations are possible : either $b$ has one end point on $\partial F_{0}$ or it has both. In the first case, $F_{0}$ is still a face of $\Gamma_{2}$, with a new factor $b b^{-1}$ in its boundary. In the second case, $F_{0}$ is cut into two faces $F_{1}$ and $F_{2}$ by $b$. Let us consider this second case. The boundaries of $F_{0}, F_{1}$ and $F_{2}$ can be written respectively $\partial F_{0}=c_{1} c_{2}, \partial F_{1}=c_{1} b^{-1}$ and $\partial F_{2}=b c_{2}$. Let $f$ be a continuous function on $G^{\Gamma_{1}}$.

$$
\begin{array}{r}
\int_{G^{\Gamma_{1}}} f d\left(\left(f_{\Gamma_{1} \Gamma_{2}}\right)_{*} P_{\beta}^{\Gamma_{2}}\right)=\frac{1}{Z_{\beta}^{\Gamma_{2}}} \int_{G^{\Gamma_{2}}} f\left(g_{1}, \ldots, g_{r}\right) p_{\sigma\left(F_{1}\right)}\left(g_{r+1}^{-1} h_{c_{1}}\right) p_{\sigma\left(F_{2}\right)}\left(h_{c_{2}} g_{r+1}\right) \\
\prod_{F \in \mathcal{F}\left(\Gamma_{1}\right) \backslash F_{0}} p_{\sigma(F)}\left(h_{\partial F}\right) d \nu_{x_{1}} \ldots d \nu_{x_{q}} d g^{\prime}
\end{array}
$$

where $g_{r+1}$ is the element associated with $b$. Since the $L_{i}$ 's are paths in $\Gamma_{1}$, the new edge $b$ is not involved in their decomposition. Thus we can isolate $d g_{r+1}$ in $d g^{\prime}$ and integrate against it. We use the fact that the heat kernel is a convolution semi-group. We get the following expression :

$$
\begin{aligned}
& =\frac{1}{Z_{\beta}^{\Gamma_{2}}} \int_{G^{\Gamma_{1}}} f\left(g_{1}, \ldots, g_{r}\right) p_{\sigma\left(F_{1}\right)+\sigma\left(F_{2}\right)}\left(h_{c_{1}} h_{c_{2}}\right) \prod_{F \in \mathcal{F}\left(\Gamma_{1}\right) \backslash F_{0}} p_{\sigma(F)}\left(h_{\partial F}\right) d \nu_{x_{1}} \ldots d \nu_{x_{q}} d g^{\prime} \\
& =\frac{Z_{\beta}^{\Gamma_{1}}}{Z_{\beta}^{\Gamma_{2}}} \int_{G^{\Gamma_{1}}} f d P_{\beta}^{\Gamma_{1}} .
\end{aligned}
$$

Setting $f=1$, we get $Z_{\beta}^{\Gamma_{1}}=Z_{\beta}^{\Gamma_{2}}$ and the result.

The case where the new edge does not cut $F_{0}$ into two faces is even simpler: the factor $b b^{-1}$ vanishes in all computations, because $f$ does not depend on the factor associated to $b$.

Now consider the case of an operation (V). Set $\Gamma_{1}=\left\{a_{1}, \ldots, a_{r}\right\}$ and $\Gamma_{2}=\left\{b, c, a_{2}, \ldots, a_{r}\right\}$, with $a_{1}=b c$. The edge $a_{1}$ can be on the boundary of one or two faces, depending on the fact that it is on $\partial M$ or not.

$$
\begin{array}{r}
\int_{G^{\Gamma_{1}}} f d\left(\left(f_{\Gamma_{1} \Gamma_{2}}\right)_{*} P_{\beta}^{\Gamma_{2}}\right)=\frac{1}{Z_{\beta}^{\Gamma_{2}}} \int_{G^{\Gamma_{2}}} f\left(g_{c} g_{b}, g_{2}, \ldots, g_{r}\right) D^{\Gamma_{1}}\left(g_{c} g_{b}, g_{2}, \ldots, g_{r}\right) \\
\nu_{x_{1}} \ldots \nu_{x_{q}} d g^{\prime}
\end{array}
$$


where $\left(g_{b}, g_{c}, g_{2}, \ldots, g_{r}\right)$ denotes the generic element of $G^{\Gamma_{2}}$. We have to discuss two cases : either $a_{1}$ is involved in the decomposition of one of the $L_{i}$ 's, say $L_{1}$, or it is not. If it is not, we can isolate $d g_{b} d g_{c}$ in the $d g^{\prime}$ term. By integrating against $d g_{b}$, the dependence in $g_{c}$ disappears by right invariance of $d g_{b}$ and we get

$$
\ldots=\frac{1}{Z_{\beta}^{\Gamma_{2}}} \int_{G^{\Gamma_{1}}} f\left(g, g_{2}, \ldots, g_{r}\right) D^{\Gamma_{1}}\left(g, g_{2}, \ldots, g_{r}\right) \nu_{x_{1}} \ldots \nu_{x_{q}} d g^{\prime}=\frac{Z_{\beta}^{\Gamma_{1}}}{Z_{\beta}^{\Gamma_{2}}} \int_{G^{\Gamma_{1}}} f d P_{\beta}^{\Gamma_{1}}
$$

and we conclude as before. If $a_{1}$ is involved in the decomposition of $L_{1}$, we can suppose that $L_{1}=a_{1} \ldots a_{m}$, with $m \geq 2$. We write $d \nu_{x_{1}}$ in a convenient way, putting the contioning on $g_{m}$, which is necessarily distinct from $g_{1}$. We get :

$$
\begin{aligned}
\frac{1}{Z_{\beta}^{\Gamma_{2}}} \int_{G^{\Gamma_{1}}} f\left(g_{c} g_{b}, g_{2}, \ldots, \widetilde{g_{m}}, \ldots, g_{r}\right) D^{\Gamma_{2}}\left(g_{c} g_{b}, g_{2}, \ldots, \widetilde{g_{m}}, \ldots, g_{r}\right) \\
d g_{b} d g_{c} d g_{2} \ldots d g_{m-1} \nu_{x_{2}} \ldots \nu_{x_{q}} d g^{\prime}
\end{aligned}
$$

where $\widetilde{g_{m}}=x_{1}\left(g_{m-1} \ldots g_{2} g_{c} g_{b}\right)^{-1}$. This is equal to

$$
\begin{aligned}
\frac{1}{Z_{\beta}^{\Gamma_{2}}} \int_{G^{\Gamma_{1}}} f\left(g, g_{2}, \ldots, \widetilde{g_{m}}, \ldots, g_{r}\right) D^{\Gamma_{2}}\left(g, g_{2}, \ldots, \widetilde{g_{m}}, \ldots, g_{r}\right) \\
\\
d g d g_{2} \ldots d g_{m-1} \nu_{x_{2}} \ldots \nu_{x_{q}} d g^{\prime}
\end{aligned}
$$

with $\widetilde{g_{m}}$ now equal to $x_{1}\left(g_{s_{1}} \ldots g_{2} g\right)^{-1}$. This is one more time equal to

$$
\frac{Z_{\beta}^{\Gamma_{1}}}{Z_{\beta}^{\Gamma_{2}}} \int_{G^{\Gamma_{1}}} f d P_{\beta}^{\Gamma_{1}}
$$

and we get the result.

Corollary 1.6.4 Let $c_{1}, \ldots, c_{n}$ be paths that are simultaneously elements of $\Gamma_{1}^{*}$ and $\Gamma_{2}^{*}$, where $\Gamma_{1}$ and $\Gamma_{2}$ are two graphs such that $\Gamma_{1}<\Gamma_{2}$. Then the law of the discrete holonomy along $c_{1}, \ldots, c_{n}$ is the same on $\Gamma_{1}$ and $\Gamma_{2}$. In other words, the law of $\left(h_{c_{1}}^{\Gamma_{1}}, \ldots, h_{c_{n}}^{\Gamma_{1}}\right)$ on $\left(G^{\Gamma_{1}}, P_{\beta}^{\Gamma_{1}}\right)$ and the law of $\left(h_{c_{1}}^{\Gamma_{2}}, \ldots, h_{c_{n}}^{\Gamma_{2}}\right)$ on $\left(G^{\Gamma_{2}}, P_{\beta}^{\Gamma_{2}}\right)$ are equal.

PROOF. It is enough to verify that $\left(h_{c_{1}}^{\Gamma_{1}}, \ldots, h_{c_{n}}^{\Gamma_{1}}\right) \circ f_{\Gamma_{1} \Gamma_{2}}=\left(h_{c_{1}}^{\Gamma_{2}}, \ldots, h_{c_{n}}^{\Gamma_{2}}\right)$. This is true if the $c_{i}$ 's are edges of $\Gamma_{1}$, thus it is true in general by multiplicativity.

During the proof of the theorem, we also proved an important result about the conditional partition function:

Proposition 1.6.5 Let $\Gamma_{1}$ and $\Gamma_{2}$ be two graphs such that $\Gamma_{1}<\Gamma_{2}$. Take $L_{1}, \ldots, L_{q}$ and $x_{1}, \ldots, x_{q}$ as usual. Then

$$
Z^{\Gamma_{1}}\left(x_{1}, \ldots, x_{q}\right)=Z^{\Gamma_{2}}\left(x_{1}, \ldots, x_{q}\right)
$$


Let us discuss briefly the choice of the heat kernel in the definition of $P$. This choice is the key of the physical relevance of the theory. It is a physicist, A. Migdal [Mi], who suggested first to use the heat kernel in the mathematical formulation of the theory. Nevertheless, it is possible to construct a discrete theory using any other convolution semigroup. For example, Albeverio, Høegh Krohn and Holden investigated some properties of the random fields obtained this way [A]. But it would probably be much more difficult to construct a continuous theory without the nice regularity properties that characterize the heat kernel among all other convolution semigroups.

\subsection{Invariance by area-preserving diffeomorphisms}

The manifold $M$ is given with its differentiable structure and the Lebesguian surface measure $\sigma$. Let $\Gamma$ be a graph on $M$ and $\phi: M \longrightarrow M$ a diffeomorphism such that $\phi_{*} \sigma=\sigma$. Then $\phi$ transforms $\Gamma$ into a graph $\phi(\Gamma)$ and induces a bijection between faces of $\Gamma$ and $\phi(\Gamma)$ that preserves the surface. Thus, the natural bijection induced between $G^{\Gamma}$ and $G^{\phi(\Gamma)}$ preserves the discrete Yang-Mills measure. Let us state in a slightly more general way this invariance property.

Proposition 1.7.1 Let $\left(M_{1}, \sigma_{1}\right)$ and $\left(M_{2}, \sigma_{2}\right)$ be two surfaces and $\phi: M_{1} \longrightarrow M_{2}$ a diffeomorphism such that $\phi_{*} \sigma_{1}=\sigma_{2}$. Let $\Gamma_{1}$ be a graph on $M_{1}$ and $\Gamma_{2}=\phi\left(\Gamma_{1}\right)$ the corresponding graph on $M_{2}$. Still denoting by $\phi: G^{\Gamma_{1}} \longrightarrow G^{\Gamma_{2}}$ the induced bijection, one has

$$
\phi_{*} P^{\Gamma_{1}}\left(x_{1}, \ldots, x_{q}\right)=P^{\Gamma_{2}}\left(x_{1}, \ldots, x_{q}\right) .
$$

Thus, for each family $\left(c_{1}, \ldots, c_{n}\right)$ of paths in $\Gamma_{1}^{*}$, the discrete holonomy along $\left(c_{1}, \ldots, c_{n}\right)$ and the discrete holonomy along $\left(\phi\left(c_{1}\right), \ldots, \phi\left(c_{n}\right)\right)$ have the same distribution.

\subsection{Examples}

In this section, we will compute the law of the discrete holonomy in two basic situations.

\subsubsection{Holonomy along an open path}

Let $\Gamma$ be a graph and $c \in \Gamma^{*}$ be an open path, i.e. a path such that $c(0) \neq c(1)$. Let $L_{1}, \ldots, L_{q}$ be disjoint simple loops of $\Gamma^{*}$ and $t_{1}, \ldots, t_{q}$ be elements of $G /$ Ad. Let us compute the law of $h_{c}$ under $P\left(t_{1}, \ldots, t_{q}\right)$. We will use the gauge invariance of $P\left(t_{1}, \ldots, t_{q}\right)$.

Let $f$ be a continuous function on $G$ and $j$ a discrete gauge transformation. Recall from the proposition 1.5 .4 that $j_{*} P\left(t_{1}, \ldots, t_{q}\right)=P\left(t_{1}, \ldots, t_{q}\right)$, so that:

$$
\begin{aligned}
\int_{G^{\Gamma}} f\left(h_{c}\right) d P\left(t_{1}, \ldots, t_{q}\right) & =\int_{G^{\Gamma}} f\left(h_{c} \circ j\right) d P\left(t_{1}, \ldots, t_{q}\right) \\
& =\int_{G^{\Gamma}} f\left(j(c(1))^{-1} h_{a} j(c(0))\right) d P\left(t_{1}, \ldots, t_{q}\right) .
\end{aligned}
$$

Thus the law of $h_{c}$ is right and left invariant on $G$ : it is the Haar measure. 


\subsubsection{Holonomy along the boundary of a small disk}

Let $\Gamma$ be a graph on $M, L_{1}, \ldots, L_{q}$ be disjoint simple loops of $\Gamma^{*}$. Let $l$ be a loop of $\Gamma^{*}$ which is the boundary of a disk $D$ such that $L_{i}([0,1])$ is not constained in $\bar{D}$ for each $i$. We will estimate the law of $h_{l}$ on $\left(G^{\Gamma}, P_{\beta}\right)$.

Let $\rho$ be the function defined on $G$ by $\rho(x)=d(1, x)$, where $d$ is the biinvariant Riemannian distance. We want to estimate the size of $\rho\left(h_{l}\right)$.

$$
\int_{G^{\Gamma}} \rho\left(h_{l}\right) d P_{\beta}=\frac{1}{Z_{\beta}} \int_{G^{\Gamma}} \rho\left(h_{l}\right) \prod_{F \in \mathcal{F}(\Gamma)} p_{\sigma(F)}\left(h_{\partial F}\right) d \nu_{x_{1}} \ldots d \nu_{x_{q}} d g^{\prime} .
$$

We need a result about graphs.

Lemma 1.8.1 Let $M$ be a surface and $\Gamma$ a graph on $M$. There exists a subgraph of $\Gamma$ which has only one face.

Proof. If a graph has more than one face, there exists an edge which is on the boundary of two different faces. If we remove this edge, the resulting pregraph is still a graph. Indeed, this removed edge was necessarily in the interior of $M$, thus boundary properties are preserved. The faces of the new pregraph are those of the old one, except two faces that were glued along a segment. So the new face is diffeomorphic to a disk and by lemma 1.2.8, we know that the new pregraph is connected. In a finite number of such steps, one gets a subgraph of $\Gamma$ with only one face.

The pregraph constituted by the $L_{i}$ 's cuts $M$ into several pieces homeomorphic to surfaces with boundary $M_{1}, \ldots, M_{k}$. The graph $\Gamma$ induces a graph on each $M_{i}$. By the preceding lemma, there exists a subgraph $\Gamma^{\prime}$ of $\Gamma$ that has exactly one face on each $M_{i}$ and such that $L_{1}, \ldots, L_{q} \in \Gamma^{* *}$. Now add to $\Gamma^{\prime}$ the edges required to form $l$ and, if necessary, a simple path connecting $l$ with $\operatorname{Supp}\left(\Gamma^{\prime}\right)$. Finally, the assumption that the image of $L_{i}$ is never contained in $\bar{D}$ shows that it is possible, maybe by adding some vertices to $\Gamma^{\prime}$, to be sure that each $L_{i}$ has an edge outside $D$. We get a graph $\Gamma^{\prime \prime}$ which is included in $\Gamma$ and in which we will compute, using the invariance by subdivision. We use the notation $P_{\beta}$ and $Z_{\beta}$.

$$
\begin{aligned}
\int_{G^{\Gamma}} \rho\left(h_{l}\right) d P_{\beta}^{\Gamma} & =\int_{G^{\Gamma^{\prime \prime}}} \rho\left(h_{l}\right) d P_{\beta}^{\Gamma^{\prime \prime}} \\
& =\frac{1}{Z_{\beta}} \int_{G^{\Gamma^{\prime \prime}}} \rho\left(h_{l}\right) \prod_{F \in \mathcal{F}\left(\Gamma^{\prime \prime}\right)} p_{\sigma(F)}\left(h_{\partial F}\right) d \nu_{x_{1}} \ldots d \nu_{x_{q}} d g^{\prime} .
\end{aligned}
$$

Lemma 1.8.2 The function $t \longrightarrow\left\|p_{t}\right\|_{\infty}$ is decreasing on $(0, \infty)$.

Proof. Let $0<s<t$ be two positive times. Let $x$ be an element of $G$. We can estimate $p_{t}(x)$ in the following way, keeping in mind that $p_{t}$ is a positive function on $G$ :

$$
p_{t}(x)=\int_{G} p_{s}\left(x y^{-1}\right) p_{t-s}(y) d y \leq\left\|p_{s}\right\|_{\infty} \int_{G} p_{t-s}(y) d y \leq\left\|p_{s}\right\|_{\infty} .
$$

Recall that each $L_{i}$ has at least an edge outside $\bar{D}$. For each $L_{i}$, we put the conditioning of $d \nu_{x_{i}}$ on one of these edges. 
On the other hand, each face $F$ of $\Gamma^{\prime \prime}$ which is not included in $D$ is included in a face of $\Gamma^{\prime}$, i.e. in a $M_{i}$ : its surface is greater than $\sigma\left(M_{i}\right)-\sigma(D)$. We assume that $\sigma(D) \leq \frac{1}{2} \inf _{i} \sigma\left(M_{i}\right)$. Then

$$
\int_{G^{\Gamma^{\prime \prime}}} \rho\left(h_{l}\right) d P_{\beta} \leq \frac{1}{Z_{\beta}} \prod_{F \not \subset D} \sup _{x \in G}\left|p_{\sigma(F)}(x)\right| \int_{G^{\Gamma^{\prime \prime}}} \rho\left(h_{l}\right) \prod_{F \subset D} p_{\sigma(F)}\left(h_{\partial F}\right) d g^{\prime}
$$

The last integral is nothing but an integral against the discrete Yang-Mills measure on $D$, which is a surface with boundary. Using the invariance by subdivision inside $D$, we can replace the graph induced by $\Gamma^{\prime \prime}$ by a very simple graph whose support is just $\partial D=l([0,1])$. This leads to

$$
\int_{G \Gamma} \rho\left(h_{l}\right) d P_{\beta}^{\Gamma} \leq \frac{1}{Z_{\beta}} \prod_{i=1}^{k}\left\|p_{\frac{\sigma\left(M_{i}\right)}{2}}\right\|_{\infty} \int_{G} \rho(g) p_{\sigma(D)}(g) d g .
$$

We are led to a problem of estimation of the heat kernel at small time.

Lemma 1.8.3 The following estimates hold:

$$
\int_{G} \rho(g)^{4} p_{t}(g) d g=O\left(t^{2}\right), \quad \int_{G} \rho(g)^{2} p_{t}(g) d g=O(t), \quad \int_{G} \rho(g) p_{t}(g) d g=O(\sqrt{t}) .
$$

Let $d$ denote the dimension of $G$. We use the following result proved in $\mathrm{Va}$ (V.4.3):

Proposition 1.8.4 There exists a positive constant $C$ such that for all $t \in(0,1)$, all $g \in G$,

$$
\frac{1}{C} t^{-\frac{d}{2}} e^{-\frac{C \rho(g)^{2}}{t}} \leq p_{t}(g) \leq C t^{-\frac{d}{2}} e^{-\frac{\rho^{2}(g)}{C t}}
$$

Proof. The first estimate implies both others. We use normal coordinates at the identity of $G$. Let $D_{R}$ be a geodesic disk of radius $R$ around 1, with $R$ such that exp is a diffeomorphism from $B(0, R) \subset T_{1} G$ onto $D_{R}$. We cut the integral according to $G=D_{R} \cup D_{R}^{c}$. One $D_{R}^{c}$, we have:

$$
\int_{D_{R}^{c}} \rho(g)^{4} p_{t}(g) d g \leq C t^{-\frac{d}{2}} \operatorname{diam}(G)^{4} e^{-\frac{R^{2}}{C t}} \leq C_{1} t^{-\frac{d}{2}} e^{-\frac{R^{2}}{C t}}
$$

For the part corresponding to $D_{R}$, we use spherical coordinates $(r, \theta)$ on $\exp ^{-1}\left(D_{R}\right)=B(0, R)$. Note that on $B(0, R)$, the image of the Haar measure by $\exp ^{-1}$ can be compared to the Lebesgue measure, so:

$$
\begin{aligned}
\int_{D_{R}} \rho(g){ }^{4} p_{t}(g) d g & \leq C_{2} \int_{[0, R] \times S^{d-1}} r^{4} p_{t}(\exp (r, \theta)) r^{d-1} d r d \theta \\
& \leq C_{3} t^{-\frac{d}{2}} \int_{0}^{R} r^{d+3} e^{-\frac{r^{2}}{C t}} d r \\
& \leq C_{3} t^{2+\frac{d}{2}} t^{-\frac{d}{2}} \int_{0}^{\infty} \frac{r^{d+3}}{t^{\frac{d+3}{2}}} e^{-\frac{r^{2}}{C t}} \frac{d r}{t^{\frac{1}{2}}} \\
& \leq C_{4} t^{2}
\end{aligned}
$$

This estimation remains true if we replace $R$ by $R^{\prime}<R$. Thus, for $t$ small enough, we have:

$$
\begin{aligned}
\int_{G} \rho(g)^{4} p_{t}(g) d g & =\int_{D_{t^{1 / 4}}} \rho(g)^{4} p_{t}(g) d g+\int_{D_{t^{1 / 4}}^{c}} \rho(g)^{4} p_{t}(g) d g \\
& \leq C_{4} t^{2}+C_{1} t^{-\frac{d}{2}} e^{-\frac{1}{C t^{1 / 2}}}=O\left(t^{2}\right) .
\end{aligned}
$$

Finally, we deduce from relation 1.6 and the preceding lemma the following proposition: 
Proposition 1.8.5 Let $\Gamma$ be a graph on $M$. Let $L_{1}, \ldots, L_{q}$ be disjoint simple loops of $\Gamma^{*}$ and $x_{1}, \ldots, x_{q}$ be elements of $G$. Let $l$ be the boundary of a disk $D$ such that none of the $L_{i}$ 's has its image contained in $\bar{D}$. There exist two positive constants $s$ and $C$ depending on the $L_{i}$ 's but not on the $x_{i}$ 's such that if $\sigma(D) \leq s$, then

$$
\int_{G^{\Gamma}} \rho\left(h_{l}\right) d P\left(x_{1}, \ldots, x_{q}\right) \leq C \sqrt{\sigma(D)} .
$$

This regularity property will play an essential role in the construction of the continuous measure.

\subsection{Discrete Abelian theory}

\subsubsection{Decomposition of cycles}

Until now, we only used the compactness of $G$. We will finish this first chapter with a detailed study of the case $G=U(1)$. All results could be extended without conceptual problems to the case $G=U(1)^{n}$, i.e. the general compact Abelian case, but this would also make the notations much heavier.

We fix $M, \sigma$ as usual and a graph $\Gamma$ on $M$. Our aim is to analyze the law of the family $\left(h_{c}\right)_{c \in \Gamma^{*}}$. Set $\left\{a_{1}, \ldots, a_{r}\right\}=\Gamma$. Since $G$ is Abelian, the function $h_{c}: G^{\Gamma} \longrightarrow G$ associated with a path $c$ depends only on the number of occurences of each $a_{i}$ in the decomposition of $c$, not on the order of the edges in this decomposition. In other words, the function $h_{c}$ depends only on the image of $c$ by the natural morphism of monoids $\Gamma^{*} \longrightarrow \mathbf{Z}^{\Gamma}$ which sends $a_{i}$ to $(0, \ldots, 1, \ldots, 0)$ with a 1 at the $i$-th place. Conversely, each element of $\mathbf{Z}^{\Gamma}$ determines without ambiguity a function from $U(1)^{\Gamma}$ into $U(1)$.

So, the natural index space in this context is $\mathbf{Z}^{\Gamma}$ instead of $\Gamma^{*}$ and this allows to consider linear combination of paths. Let us denote by $C \Gamma \subset \mathbf{Z}^{\Gamma}$ the set of linear combination of loops, also called cycles. We are especially interested in the law of $\left(h_{c}\right)_{c \in C \Gamma}$. The reason for which we consider only loops will become clear at the end of chapter 2. Basically, it is because for an arbitrary $G$, the holonomy along an open path is not a gauge-invariant function of a connection.

Let us recall a classical result about the homology of $M$.

Theorem 1.9.1 Let $g$ be the genus of $M$ and $p$ the number of connected components of $\partial M$. Then

$$
H_{1}(M ; \mathbf{Z}) \simeq\left\{\begin{array}{l}
\mathbf{Z}^{2 g} \text { if } p=0 \\
\mathbf{Z}^{2 g+p-1} \text { if } p>0
\end{array}\right.
$$

If $p>0$, one can construct a system of loops representing a basis of $H_{1}(M)$ by taking $p-1$ components of $\partial M$ and $2 g$ loops of $M$ that generate the $H_{1}$ of a minimal closure of $M$, i.e. a surface obtained from $M$ by gluing a disk along each boundary component.

So, let us choose such a system composed by $\ell_{1}, \ldots, \ell_{2 g}$ in $\Gamma^{*}$ and $p-1$ loops $N_{1}, \ldots, N_{p-1}$ that we denote just as the corresponding boundary components, with an abuse of notation. We can obtain the $\ell_{i}$ 's by deforming an arbitrary system of generators using the same technique as in the proof of the proposition 1.2.4.

Now let $c$ be a cycle in $C \Gamma$. There is an unique decomposition

$$
c=\lambda_{1} \ell_{1}+\ldots+\lambda_{2 g} \ell_{2 g}+\nu_{1} N_{1}+\ldots+\nu_{p-1} N_{p-1}+c^{\perp},
$$


with $\lambda_{i}, \nu_{j} \in \mathbf{Z}$ and $c^{\perp} \in C \Gamma$ a cycle homologous to zero. Let us denote by $C_{0} \Gamma$ the submodule of $C \Gamma$ spanned by the cycles homologous to zero.

Proposition 1.9.2 If $\partial M$ is empty (resp. non empty), the boundaries of all faces except one chosen arbitrarily (resp. of all faces) form a basis of the submodule $C_{0} \Gamma$ of $C \Gamma$.

We will prove this proposition very soon. Set $\mathcal{F}(\Gamma)=\left\{F_{1}, \ldots, F_{n}\right\}$ and choose for each $F_{i}$ a cycle $\partial F_{i}$ whose image is the boundary of $F_{i}$. We can write :

$$
c=\lambda_{1} \ell_{1}+\ldots+\lambda_{2 g} \ell_{2 g}+\nu_{1} N_{1}+\ldots+\nu_{p-1} N_{p-1}+\mu_{1} \partial F_{1}+\ldots+\mu_{n} \partial F_{n},
$$

the decomposition being non unique if $M$ is closed. The relation 1.7, together with the multiplicativity of the holonomy, shows that the law of the family $\left(h_{c}\right)_{c \in C \Gamma}$ is completely determined by the law of what we will call a fundamental system :

$$
\left(h_{\ell_{1}}, \ldots, h_{\ell_{2 g}}, h_{N_{1}}, \ldots, h_{N_{p-1}}, h_{\partial F_{1}}, \ldots, h_{\partial F_{n}}\right) .
$$

Proofof proposition 1.9.2 : To begin with, suppose that $M$ has no boundary. We proceed by induction on $n=\operatorname{Card} \mathcal{F}(\Gamma)$. If $n=1$, the only loop in $C \Gamma$ is $\partial F$ and it is homologically trivial.

Now suppose that the result is true for a graph with $n-1$ faces. Let $\Gamma$ be a graph with $n$ faces. There is an edge of $\Gamma$, say $a_{r}$, which is on the boundary of two distinct faces, say $F_{n-1}$ and $F_{n}$. Let $\Gamma^{\prime}=\left\{a_{1}, \ldots, a_{r-1}\right\}$ be the graph obtained by removing $a_{r}$. It has $n-1$ faces $F_{1}, \ldots, F_{n-2}, F_{n-1} \cup F_{n}$. Let $c$ be a cycle of $C_{0} \Gamma$. We can decompose it uniquely in $c=c_{0}+p a_{r}$ with $p \in \mathbf{Z}$ and $c_{0} \in C \Gamma^{\prime}$. We can also write $\partial F_{n-1}=a_{r}+b$ with $b \in C \Gamma^{\prime}$. So , we have $c=\left(c_{0}-p b\right)+p \partial F_{n-1}$. By induction, $c_{0}-p b$, which is homologous to zero in $\Gamma^{\prime}$, is a linear combination of $\partial F_{1}, \ldots, \partial F_{n-2}$. Thus, $\partial F_{1}, \ldots, \partial F_{n-1}$ generate the submodule of homologically trivial cycles in $C \Gamma$. On the other hand, $\partial F_{1}, \ldots, \partial F_{n-2}$ are linearly indendent by induction and $\partial F_{n-1}$ is independent of the submodule that they generate, because it contains the edge $a_{r}$. This gives the result when $M$ is closed.

If $M$ has a boundary, consider a minimal closure $i_{1}: M \longrightarrow M_{1}$ of $M$ and identify $M$ with $i_{1}(M)$. Let $c$ be a cycle homologous to zero in $M$. It is also homologous to zero in $M_{1}$ and can be decomposed using the result on $M_{1}$ into :

$$
c=\sum_{F_{i} \in \mathcal{F}(\Gamma), F_{i} \subset M} \mu_{i} \partial F_{i}+\nu_{1} N_{1}+\ldots+\nu_{p-1} N_{p-1},
$$

because the $N_{i}$ 's are the boundaries of the faces of $\Gamma$ on $M_{1}-M$. This decomposition gives, in $H_{1}(M)$,

$$
[c]=0=\nu_{1}\left[N_{1}\right]+\ldots+\nu_{p-1}\left[N_{p-1}\right]
$$

implying $\nu_{1}=\ldots=\nu_{p-1}=0$ and $c=\mu_{1} \partial F_{1}+\ldots+\mu_{n} \partial F_{n}$. The independence of the $\partial F_{i}$ 's on $M_{1}$ implies their independence on $M$.

\subsubsection{Study of a fundamental system}

We want to study the discrete Yang-Mills measure conditioned by the holonomies along the boundary components of $M$. Let $x_{1}, \ldots, x_{p}$ be elements of $U(1)$. Under $P_{\beta}=P\left(x_{1}, \ldots, x_{p}\right)$, the law of $\left(h_{N_{1}}, \ldots, h_{N_{p-1}}\right)$ is deterministic, equal to $\delta_{\left(x_{1}, \ldots, x_{p-1}\right)}$. 
Proposition 1.9.3 Under the measure $\nu_{x_{1}} \otimes \ldots \otimes \nu_{x_{q}} \otimes d g^{\prime}$ on $G^{\Gamma}$, the variables $h_{\ell_{1}}, \ldots, h_{\ell_{2 g}}$, $h_{\partial F_{1}}, \ldots, h_{\partial F_{n-1}}$ are uniform and independent on $U(1)$.

Proof. We compute the characteristic function of $\left(h_{\ell_{1}}, \ldots, h_{\ell_{2 g}}, h_{\partial F_{1}}, \ldots, h_{\partial F_{n-1}}\right)$, seen as a $\mathbf{C}^{2 g+n-1}$-valued random variable. In order to simplify the notations, we choose an orientation of $M$ and assume that each $N_{i} \subset \partial M$ and each $\partial F_{i}$ is oriented according to the usual convention. Let $\lambda_{1}, \ldots, \lambda_{2 g}, \mu_{1}, \ldots, \mu_{n-1}$ be integers.

$$
\begin{aligned}
F\left(\lambda_{1}, \ldots, \lambda_{2 g}, \mu_{1}, \ldots, \mu_{n-1}\right) & =\int_{U(1)^{\Gamma}} h_{\ell_{1}}^{\lambda_{1}} \ldots h_{\ell_{2 g}}^{\lambda_{2 g}} h_{\partial F_{1}}^{\mu_{1}} \ldots h_{\partial F_{n-1}}^{\mu_{n-1}} d P_{\beta} \\
& =\int_{U(1)^{\Gamma}} h_{\lambda_{1} \ell_{1}+\ldots+\lambda_{2 g} \ell_{2 g}+\mu_{1} \partial F_{1}+\ldots+\mu_{n-1} \partial F_{n-1}} d P_{\beta} \\
& =\int_{U(1)^{\Gamma}} h_{a_{1}}^{\alpha_{1}} \ldots h_{a_{r}}^{\alpha_{r}} d P_{\beta},
\end{aligned}
$$

where $\sum_{i} \lambda_{i} \ell_{i}+\sum_{j} \mu_{j} \partial F_{j}=\sum_{k} \alpha_{k} a_{k}$. Suppose that the $a_{i}$ 's are labeled in such a way that $N_{1}=a_{1} \ldots a_{i_{1}}, \ldots, N_{p}=a_{i_{p-1}+1} \ldots a_{i_{p}}$ with $1<a_{1}<\ldots<a_{i_{p}}$.

$$
\begin{aligned}
F\left(\lambda_{i}, \mu_{j}\right) & =\int_{U(1)^{\Gamma}}\left(h_{a_{1}}^{\alpha_{1}} \ldots h_{a_{i_{1}}}^{\alpha_{i_{1}}}\right) \ldots\left(h_{a_{i_{p-1}+1}}^{\alpha_{i_{p-1}+1}} \ldots h_{a_{i_{p}}}^{\alpha_{i_{p}}}\right) h_{a_{i_{p}+1}}^{\alpha_{i_{p}+1}} \ldots h_{a_{r}}^{\alpha_{r}} d P_{\beta} \\
& =\int_{U(1)^{\Gamma}} g_{1}^{\alpha_{1}} \ldots g_{i_{1}}^{\alpha_{i_{1}}} d \nu_{x_{1}} \ldots \int_{U(1)^{\Gamma}} g_{i_{p-1}+1}^{\alpha_{i_{p-1}+1}} \ldots g_{i_{p}}^{\alpha_{i_{p}}} d \nu_{x_{p}} \\
& \int_{U(1)^{\Gamma}} g^{\alpha_{i_{p}+1}} d g \ldots \int_{U(1)^{\Gamma}} g^{\alpha_{r}} d g .
\end{aligned}
$$

This product is zero if one of the $\alpha_{k}$ 's with $k \geq i_{p}+1$ is nonzero. Otherwise, $\partial M$ is non empty and the cycle $\sum \lambda_{i} \ell_{i}+\sum \mu_{j} \partial F_{j}$ has all its edges on $\partial M$. Thus, we have an equality

$$
\sum_{i=1}^{2 g} \lambda_{i} \ell_{i}+\sum_{j=1}^{n-1} \mu_{j} \partial F_{j}=\sum_{k=1}^{p} \nu_{k} N_{k},
$$

which, in $H_{1}(M)$, implies $\nu_{p}\left[N_{p}\right]=\sum \lambda_{i}\left[\ell_{i}\right]-\sum_{k<p} \nu_{k}\left[N_{k}\right]$. Since $\left[N_{p}\right]=-\left[N_{1}\right]-\ldots-\left[N_{p-1}\right]$, this implies $\lambda_{i}=0$ for all $i$ and $\nu_{k}=\nu_{p}$ for all $k$. We get

$$
\sum_{j=1}^{n-1} \mu_{j} \partial F_{j}=\nu \sum_{i=1}^{p} N_{i}=\nu \sum_{j=1}^{n} \partial F_{j} .
$$

Since $\partial F_{1}, \ldots, \partial F_{n}$ are independent, the comparison of the coefficients of the $\partial F_{n}$ 's gives $\nu=0$ and then $\mu_{j}=0$. Finally, the cycle $\sum \lambda_{i} \ell_{i}+\sum \mu_{j} \partial F_{j}$ is equal to zero and $F\left(\lambda_{i}, \mu_{j}\right)=1$. Thus, we proved that $F\left(\lambda_{i}, \mu_{j}\right)$ is equal to zero, except if all $\lambda_{i}$ 's and $\mu_{j}$ 's are zero, in which case it is equal to 1 . This proves the result.

The last element to study in the fundamental system is $h_{\partial F_{n}}$. We have

$$
\partial F_{n}=\sum_{i=1}^{p} N_{i}-\sum_{j=1}^{n-1} \partial F_{j},
$$

so that $h_{\partial F_{n}}=x_{1} \ldots x_{p} h_{\partial F_{1}}^{-1} \ldots h_{\partial F_{n-1}}^{-1}$ under $\nu_{x_{1}} \otimes \ldots \otimes \nu_{x_{p}} \otimes d g^{\prime}$. 
Proposition 1.9.4 Set $x=x_{1} \ldots x_{p}$ if $M$ has a boundary and $x=1$ if $M$ is closed. For any function $f$ continuous on $G^{2 g+n+p}$,

$$
\begin{gathered}
\int_{G^{\Gamma}} f\left(h_{\ell_{1}}, \ldots, h_{\ell_{2 g}}, h_{N_{1}}, \ldots, h_{N_{p-1}}, h_{\partial F_{1}}, \ldots, h_{\partial F_{n}}\right) d P\left(x_{1}, \ldots, x_{p}\right)= \\
\int_{G^{2 g+n}} f\left(u_{1}, \ldots, u_{2 g}, x_{1}, \ldots, x_{p-1}, v_{1}, \ldots, v_{n}\right) p_{\sigma\left(F_{1}\right)}\left(v_{1}\right) \ldots p_{\sigma\left(F_{n}\right)}\left(v_{n}\right) \\
d u_{1} \ldots d u_{2 g} d \nu_{x}^{n}\left(v_{1}, \ldots, v_{n}\right) .
\end{gathered}
$$

Note that, in the Abelian setting, the measure $\nu_{x}^{n}$ is invariant by permutations of the factors in $U(1)^{n}$.

\subsubsection{Gaussian aspect of the Abelian theory}

We proved that the law of the whole family $\left(h_{c}\right)_{c \in C \Gamma}$ is determined by the law of a fundamental system and we just described this law. So we could consider that the proposition 1.9.4 is the answer to our question. In fact, it is possible to be much more explicit by taking the gaussian character of the Abelian theory into account. The crucial part of the law of a fundamental system is of course that of $\left(h_{\partial F_{1}}, \ldots, h_{\partial F_{n}}\right)$. We will concentrate on this law.

Proposition 1.9.5 Let $Y_{1}, \ldots, Y_{n}$ be independent centered real gaussian random variables with $Y_{i} \sim \mathcal{N}\left(0, \sigma\left(F_{i}\right)\right)$. Let $S=Y_{1}+\ldots+Y_{n}$ be their sum. For each $i=1, \ldots, n$, set

$$
X_{i}=Y_{i}-\frac{\sigma\left(F_{i}\right)}{\sigma(M)} S
$$

Let $T$ be a real random variable, independent of the $Y_{i}$ 's, with the following discrete law :

$$
P(T=t)=\left\{\begin{array}{l}
\left(\sum_{s, e^{i s}=x} e^{-\frac{s^{2}}{2 \sigma(M)}}\right)^{-1} e^{-\frac{t^{2}}{2 \sigma(M)}} \text { if } e^{i t}=x \\
0 \text { otherwise, }
\end{array}\right.
$$

where, as before, $x=x_{1} \ldots x_{p}$ if $M$ has a boundary and $x=1$ if $M$ is closed. Then, for any function $f$ continuous on $G^{n}$,

$$
\int_{G^{\Gamma}} f\left(h_{\partial F_{1}}, \ldots, h_{\partial F_{n}}\right) d P_{\beta}=E f\left(e^{i\left(X_{1}+\frac{\sigma\left(F_{1}\right)}{\sigma(M)} T\right)}, \ldots, e^{i\left(X_{n}+\frac{\sigma\left(F_{n}\right)}{\sigma(M)} T\right)}\right)
$$

The law of $T$ described in this theorem is just that of a $\mathcal{N}(0, \sigma(M))$ random variable conditioned to take its values in $\exp ^{-1}(x)$, where $\exp (t)=e^{i t}$. We shall discuss the meaning of this variable in section 3.2.2.

Proof. In this proof, we set $\sigma_{i}=\sigma\left(F_{i}\right)$ and $\sigma_{M}=\sigma(M)$. One easily computes

$$
E X_{i} X_{j}=\delta_{i j} \sigma_{i}-\frac{\sigma_{i} \sigma_{j}}{\sigma_{M}}
$$

and $\sum X_{i}=0$ a.s. The law of $\left(X_{1}, \ldots, X_{n}\right)$ has no density with respect to Lebesgue measure on $\mathbf{R}^{n}$, but that of $\left(X_{1}, \ldots, X_{n-1}\right)$ does, on $\mathbf{R}^{n-1}$. Denote by $C$ the $(n-1) \times(n-1)$ covariance matrix of $\left(X_{1}, \ldots, X_{n-1}\right)$. One easily checks that $C^{-1}$ is given by

$$
\left(C^{-1}\right)_{i j}=\frac{\delta_{i j}}{\sigma_{i}}+\frac{1}{\sigma_{n}} .
$$


So the density of the law of $\left(X_{1}, \ldots, X_{n}\right)$ is :

$$
d \eta\left(t_{1}, \ldots, t_{n-1}\right)=\frac{1}{Z} \exp -\frac{1}{2}\left(\sum_{i=1}^{n-1} \frac{t_{i}^{2}}{\sigma_{i}}+\sum_{i, j=1}^{n-1} \frac{t_{i} t_{j}}{\sigma_{n}}\right) d t_{1} \ldots d t_{n} .
$$

Let us fix a number $t_{0}$ such that $e^{i t_{0}}=x$. We also set $t_{n}=-t_{1}-\ldots-t_{n-1}$. We can compute the right term of (1.8): it is equal to

$$
\begin{aligned}
& \frac{1}{Z} \sum_{k \in \mathbf{Z}} \int_{\mathbf{R}^{n-1}} f\left(e^{i t_{1}+\frac{\sigma_{1}}{\sigma_{M}}\left(t_{0}+2 k \pi\right)}, \ldots, e^{i t_{n-1}+\frac{\sigma_{n-1}}{\sigma_{M}}\left(t_{0}+2 k \pi\right)}, e^{i t_{n}+\frac{\sigma_{n}}{\sigma_{M}}\left(t_{0}+2 k \pi\right)}\right) \\
= & \frac{1}{Z} \sum_{k, q_{1}, \ldots, q_{n-1} \in \mathbf{Z}} \int_{[0,2 \pi]^{n-1}} f\left(e^{i t_{1}}, \ldots, e^{i t_{n-1}}, e^{i\left(t_{n}+t_{0}\right)}\right) \\
& \left.\exp \left(-\frac{1}{2} \sum_{i=1}^{n-1} \frac{t_{i}^{2}}{\sigma_{i}}+\sum_{i, j=1}^{n-1} \frac{t_{i} t_{j}}{\sigma_{n}}\right) \exp \frac{-\left(t_{0}+2 k \pi\right)^{2}}{2 \sigma_{M}} d t_{1} \ldots d t_{n-1}+2 \pi q_{i}-\frac{\sigma_{i}}{\sigma_{M}}\left(t_{0}+2 k \pi\right)\right)^{2}+ \\
& \left.+\frac{1}{2 \sigma_{n}}\left(\sum_{i=1}^{n-1} t_{i}+2 \pi q_{i}-\frac{\sigma_{i}}{\sigma_{M}}\left(t_{0}+2 k \pi\right)\right)^{2}\right) \exp -\frac{\left(t_{0}+2 k \pi\right)^{2}}{2 \sigma_{M}} d t_{1} \ldots d t_{n-1} .
\end{aligned}
$$

We do not care about normalization constants, since two probability measures with proportional densities are equal. Now we compute the left hand side of (1.8) using the following expression of the heat kernel :

$$
p_{s}\left(e^{i t}\right)=\frac{1}{Z} \sum_{p \in \mathbf{Z}} e^{-\frac{(t-2 p \pi)^{2}}{2 s}}
$$

which is just the image by the exponential map of the heat kernel on $\mathbf{R}$. We get

$$
\begin{array}{r}
\int_{G^{\Gamma}} f\left(h_{\partial F_{1}}, \ldots, h_{\partial F_{n}}\right) d P_{\beta}=\int_{G^{n}} f\left(v_{1}, \ldots, v_{n}\right) p_{\sigma_{1}}\left(v_{1}\right) \ldots p_{\sigma_{n}}\left(v_{n}\right) \nu_{x}^{n}\left(v_{1}, \ldots, v_{n}\right) \\
=\sum_{p_{1}, \ldots, p_{n} \in \mathbf{Z}} \int_{[0,2 \pi]^{n-1}} f\left(e^{i t_{1}}, \ldots, e^{i t_{n-1}}, e^{i\left(t_{n}+t_{0}\right)}\right) \exp \left(-\frac{1}{2} \sum_{i=1}^{n-1} \frac{\left(t_{i}-2 p_{i} \pi\right)^{2}}{\sigma_{i}}\right) \\
\exp \left(-\frac{1}{2} \frac{\left(t_{n}+t_{0}-2 p_{n} \pi\right)^{2}}{\sigma_{n}}\right) d t_{1} \ldots d t_{n-1}
\end{array}
$$

The result will be a consequence of the following equality:

$$
\begin{aligned}
& \sum_{p_{1}, \ldots, p_{n} \in \mathbf{Z}} \exp \left(-\frac{1}{2} \sum_{i=1}^{n-1} \frac{\left(t_{i}-2 p_{i} \pi\right)^{2}}{\sigma_{i}}-\right.\left.\frac{1}{2} \frac{\left(t_{n}+t_{0}-2 p_{n} \pi\right)^{2}}{\sigma_{n}}\right)= \\
& \sum_{k, q_{1}, \ldots, q_{n-1} \in \mathbf{Z}} \exp \frac{-\left(t_{0}+2 k \pi\right)^{2}}{2 \sigma_{M}}-\frac{1}{2} \sum_{i=1}^{n-1} \frac{1}{\sigma_{i}}\left(t_{i}+2 \pi q_{i}-\frac{\sigma_{i}}{\sigma_{M}}\left(t_{0}+2 k \pi\right)\right)^{2}- \\
&-\frac{1}{2 \sigma_{n}}\left(\sum_{i=1}^{n-1} t_{i}+2 \pi q_{i}-\frac{\sigma_{i}}{\sigma_{M}}\left(t_{0}+2 k \pi\right)\right)^{2}
\end{aligned}
$$


Setting $q_{n}=-q_{1}-\ldots-q_{n-1}$, we have

$$
\begin{aligned}
& \sum_{i=1}^{n-1} \frac{1}{\sigma_{i}}\left(t_{i}+2 \pi q_{i}-\frac{\sigma_{i}}{\sigma_{M}}\left(t_{0}+2 k \pi\right)\right)^{2}+\frac{1}{\sigma_{n}}\left(\sum_{i=1}^{n-1} t_{i}+2 \pi q_{i}-\frac{\sigma_{i}}{\sigma_{M}}\left(t_{0}+2 k \pi\right)\right)^{2}= \\
& =\sum_{i=1}^{n-1} \frac{1}{\sigma_{i}}\left(\left[t_{i}+2 \pi q_{i}\right]-\frac{\sigma_{i}}{\sigma_{M}}\left(t_{0}+2 k \pi\right)\right)^{2}+ \\
& +\frac{1}{\sigma_{n}}\left(\left[-t_{n}-2 \pi q_{n}-\left(t_{0}+2 k \pi\right)\right]+\frac{\sigma_{n}}{\sigma_{M}}\left(t_{0}+2 k \pi\right)\right)^{2} \\
& =\sum_{i=1}^{n-1} \frac{1}{\sigma_{i}}\left[\left(t_{i}+2 \pi q_{i}\right)^{2}-\frac{2 \sigma_{i}}{\sigma_{M}}\left(t_{i}+2 \pi q_{i}\right)\left(t_{0}+2 k \pi\right)+\frac{\sigma_{i}^{2}}{\sigma_{M}^{2}}\left(t_{0}+2 k \pi\right)^{2}\right]+ \\
& +\frac{1}{\sigma_{n}}\left[\left(-t_{n}-2 \pi q_{n}-\left(t_{0}+2 k \pi\right)\right)^{2}+\frac{\sigma_{n}^{2}}{\sigma_{M}^{2}}\left(t_{0}+2 k \pi\right)^{2}+\right. \\
& \left.+\frac{2 \sigma_{n}}{\sigma_{M}}\left(-t_{n}-2 \pi q_{n}-\left(t_{0}+2 k \pi\right)\right)\left(t_{0}+2 k \pi\right)\right] \\
& =\sum_{i=1}^{n-1} \frac{1}{\sigma_{i}}\left(t_{i}+2 \pi q_{i}\right)^{2}+\frac{1}{\sigma_{M}}\left(t_{0}+2 k \pi\right)^{2}\left(\sum_{i=1}^{n-1} \frac{\sigma_{i}}{\sigma_{M}}+\frac{\sigma_{n}}{\sigma_{M}}\right) \\
& -\frac{2}{\sigma_{M}} \sum_{i=1}^{n-1}\left(t_{i}+2 \pi q_{i}\right)\left(t_{0}+2 k \pi\right)+\frac{2}{\sigma_{M}} \sum_{i=1}^{n-1}\left(t_{i}+2 \pi q_{i}\right)\left(t_{0}+2 k \pi\right) \\
& -\frac{2}{\sigma_{M}}\left(t_{0}+2 k \pi\right)^{2}+\frac{1}{\sigma_{n}}\left(-t_{n}-2 \pi q_{n}-\left(t_{0}+2 k \pi\right)\right)^{2} \\
& =\sum_{i=1}^{n-1} \frac{1}{\sigma_{i}}\left(t_{i}+2 \pi q_{i}\right)^{2}+\frac{1}{\sigma_{n}}\left(t_{n}+t_{0}+2 \pi q_{n}+2 k \pi\right)^{2}-\frac{1}{\sigma_{M}}\left(t_{0}+2 k \pi\right)^{2} .
\end{aligned}
$$

Setting $p_{1}=q_{1}, \ldots, p_{n-1}=q_{n-1}$ and $p_{n}=-q_{n}-k$, we get the result.

\subsubsection{The double layer potential}

To go further, we would like to represent isometrically the vector $\left(X_{1}, \ldots, X_{n}\right)$ by a vector of functions of $L^{2}(M, \sigma)$ naturally associated with $F_{1}, \ldots, F_{n}$. To begin with, remark that the vector $\left(\mathbf{1}_{F_{1}}, \ldots, \mathbf{1}_{F_{n}}\right)$ has the same covariance as $\left(Y_{1}, \ldots, Y_{n}\right)$. Now set

$$
u_{i}=\mathbf{1}_{F_{i}}-\frac{\sigma\left(F_{i}\right)}{\sigma(M)}
$$

The vector $\left(u_{1}, \ldots, u_{n}\right)$ has the same covariance as $\left(X_{1}, \ldots, X_{n}\right)$. Each $u_{i}$ can be seen as the orthogonal projection of $\mathbf{1}_{F_{i}}$ on the hyperplane $L_{0}^{2}(M, \sigma)$ of functions whose mean is equal to zero. In fact, the $u_{i}$ 's are the most natural generalizations on $M$ of the classical index of a loop around a point in the plane. We will give a more direct definition of $u_{i}$.

To do this, we endow $M$ with a Riemannian metric whose volume coincides with $\sigma$. There exist a lot of such metrics, because $\sigma$ is equivalent to the Lebesgue measure in any chart with a smooth density, as well as the Riemannian volume of any Riemannian metric on $M$. 
The choice of a compatible metric on $M$ gives rise to a Laplace operator $\Delta$ and to a Hodge operator $*$ on $\bigwedge^{1}\left(T^{*} M\right)$. There exists on $M$ a Green function $G: M \times M \longrightarrow \mathbf{R}_{+}$defined outside the diagonal which is symmetric, smooth and such that

$$
\left\{\begin{array}{l}
\Delta G(x, \cdot)=\delta_{x}-\frac{1}{\sigma(M)} \forall x \in M \\
\int_{M} G(x, y) d \sigma(y)=0 \quad \forall x \in M \text { when } \partial M=\emptyset \\
* d G_{x}=0 \text { on } \partial M \quad \forall x \in M \text { when } \partial M \neq \emptyset,
\end{array}\right.
$$

where $G_{x}$ denotes the function $G(x, \cdot)$. A proof of this fact can be found in Au . Note that when $M$ has a boundary, there exists a solution to $\Delta \widetilde{G}_{x}=\delta_{x}$. Nevertheless, this choice would be incompatible with the condition $* d G_{x}=0$ on $\partial M$ which implies $\int_{M} \Delta G_{x}=0$.

Definition 1.9.6 Let $c$ be a path on $M$. We call double layer potential of $c$ the function $u_{c}$ defined on $M$ outside the image of $c$ by :

$$
u_{c}(x)=\int_{c} * d G_{x} .
$$

Note that the double layer potential is additive: if $c_{1}$ and $c_{2}$ are two cycles of $C \Gamma$, then $u_{c_{1}+c_{2}}=u_{c_{1}}+u_{c_{2}} \sigma$-a.e. on $M$.

Proposition 1.9.7 Let $c$ be a simple loop which is the boundary of a subset $U$ of $M$. Set $V=U^{c}$. Then

$$
u_{c}(x)=\frac{\sigma(V)}{\sigma(M)} \mathbf{1}_{U}(x)-\frac{\sigma(U)}{\sigma(M)} \mathbf{1}_{V}(x)=\mathbf{1}_{U}-\frac{\sigma(U)}{\sigma(M)} .
$$

In particular, $u_{c} \in L^{2}(M, \sigma)$ and $\left\|u_{c}\right\|_{2}=\left(\frac{\sigma(U) \sigma(V)}{\sigma(M)}\right)^{\frac{1}{2}}$.

Proof. Let $x$ be in $U$. Since $* d G_{x}=0$ on $\partial M$, we have:

$$
u_{c}(x)=\int_{\partial U} * d G_{x}=-\int_{\partial V} * d G_{x}=-\int_{V} \delta_{x}-\frac{1}{\sigma(M)}=\frac{\sigma(V)}{\sigma(M)} .
$$

Now let $x$ be in $V$.

$$
u_{c}(x)=\int_{\partial U} * d G_{x}=\int_{U} \delta_{x}-\frac{1}{\sigma(M)}=-\frac{\sigma(U)}{\sigma(V)} .
$$

The last part of the statement follows easily.

Corollary 1.9.8 The vector $\left(u_{1}, \ldots, u_{n}\right)$ is equal to $\left(u_{\partial F_{1}}, \ldots, u_{\partial F_{n}}\right)$.

To go from functions $u_{\partial F_{i}}$ to random variables $X_{i}$, we need an isometry of $L^{2}(M, \sigma)$ into a gaussian space, in other words a white noise on $(M, \sigma)$. Let us consider a white noise

$$
\begin{aligned}
W: L^{2}(M, \sigma) & \longrightarrow \mathcal{G} \\
u & \longmapsto W(u)
\end{aligned}
$$

such that for any $u, v \in L^{2}(M), W(u)$ and $W(v)$ are real centered gaussian random variables such that $E[W(u) W(v)]=(u, v)_{L^{2}}$. The proposition 1.9.5 can be rewritten in the following form: 
Proposition 1.9.9 The following equality holds in law:

$$
\left(h_{\partial F_{1}}, \ldots, h_{\partial F_{n}}\right) \stackrel{\operatorname{law}}{=}\left(e^{i\left(W\left(u_{\partial F_{1}}\right)+\frac{\sigma\left(F_{1}\right)}{\sigma(M)} T\right)}, \ldots, e^{i\left(W\left(u_{\partial F_{n}}\right)+\frac{\sigma\left(F_{n}\right)}{\sigma(M)} T\right)}\right) .
$$

We would like to extend this result to arbitrary cycles homologous to zero. Let $c_{1}, \ldots c_{k}$ be cycles of $C_{0} \Gamma$. For each $i, c_{i}$ is a linear combination of the $\partial F_{i}$ thus $u_{c_{i}}$ is well defined and is in $L^{2}(M)$. So, $W\left(u_{c_{i}}\right)$ is well defined.

We have to generalize the term $\frac{\sigma\left(F_{i}\right)}{\sigma(M)}$. Since $c_{i}$ is homologous to zero, it is the boundary of a two-chain denoted by $\alpha$. If $M$ has a boundary, $H_{2}(M)=0$ and $\alpha$ is well defined by $\partial \alpha=c$. We identify $\sigma$ with a 2 -form on $M$ and set $\sigma(\alpha)=|\langle\sigma, \alpha\rangle|$, using the natural pairing between 2 -forms and 2-chains. So the number

$$
\sigma_{\text {int }}(c)=\frac{\sigma(\alpha)}{\sigma(M)}
$$

is well defined. If $M$ is closed, $H_{2}(M) \simeq \mathbf{Z}$ and $\alpha$ is defined up to a multiple of $[M]$. So the number $\sigma_{\text {int }}(c)$ is only defined modulo 1 . But in this case, $T$ takes its values in $\exp ^{-1}(1)=2 \pi \mathbf{Z}$ so that $\exp i \sigma_{\text {int }}(c) T$ is well defined.

Proposition 1.9.10 Let $\left(c_{1}, \ldots, c_{k}\right)$ be cycles of $C_{0} \Gamma$. Then the following equality in law holds:

$$
\left(h_{c_{1}}, \ldots, h_{c_{k}}\right) \stackrel{\text { law }}{=}\left(e^{i\left(W\left(u_{c_{1}}\right)+\sigma_{\mathrm{int}}\left(c_{1}\right) T\right)}, \ldots, e^{i\left(W\left(u_{c_{k}}\right)+\sigma_{\mathrm{int}}\left(c_{k}\right) T\right)}\right) .
$$

Proof. By proposition 1.9.9, the result is true when $\left(c_{1}, \ldots, c_{k}\right)=\left(\partial F_{1}, \ldots, \partial F_{n}\right)$. Since the boundaries of the faces constitute a basis of $C_{0} \Gamma$, it is sufficient to show that the new set of variables defined using the white noise satisfy the same multiplicativity property as $\left(h_{c}\right)_{c \in C_{0} \Gamma}$.

On one hand, $W$ is linear and the double layer potential is additive, so that $\exp i W\left(u_{c_{1}+c_{2}}\right)=$ $\exp i W\left(u_{c_{1}}\right) \exp i W\left(u_{c_{2}}\right)$. On the other hand, $c_{1}=\partial \alpha_{1}$ and $c_{2}=\partial \alpha_{2}$ imply $c_{1}+c_{2}=\partial\left(\alpha_{1}+\alpha_{2}\right)$, so $\sigma_{\text {int }}$ is also additive. This proves the result.

The results that we proved in this section are the starting point of the more detailed investigation that will be done in chapter 3, after the continuous Yang-Mills measure has been constructed.

Some properties of the double layer potential will be proved in the next chapter, using a favorable technical context. Nevertheless, it is necessary to state here a fundamental property that will be proved at the end of the chapter 3 .

Theorem 1.9.11 For any path $c$ of $P M$, the function $u_{c}$ is in $L^{2}(M, \sigma)$. 


\section{Chapter 2}

\section{Continuous Yang-Mills measure}

In chapter 1, we defined a random holonomy along the paths of a graph on $M$. Our aim in this chapter is to extend this definition to all paths on $M$. The problem is that there are families of paths that cannot be realized as subfamilies of any $\Gamma^{*}, \Gamma$ being a graph on $M$ : even two smooth paths can cross each other an infinity of times an give rise to an infinity of connected components on $M$, a situation in which we are unable to write the joint law of their holonomies using the tools of the preceding chapter.

The two properties of the discrete theory that are essential to our purpose are the invariance by subdivision, expressed in theorem 1.6.1 and the regularity property of the proposition 1.8.5. Our basic idea is to cover $M$ with finer and finer graphs and to prove that the discrete measures on these graphs converge in some sense to a continuous object that will be called continuous Yang-Mills measure.

\subsection{Projective systems}

Let $\left(\Gamma_{\lambda}\right)_{\lambda \in \Lambda}$ be a family of graphs on $M$, which approximates correctly $M$, whatever this means exactly. In sections 1.4 and 1.5, we explained how to construct a family of probability spaces corresponding to this family of graphs. We will now consider the projective limit of this family of probability spaces.

Definition 2.1.1 Let $\Lambda$ be an ordered set such that for all $\lambda, \mu \in \Lambda$, there exists $\nu \in \Lambda$ such that $\lambda<\nu$ and $\mu<\nu$. A projective family of probability spaces indexed by $\Lambda$ is a family $\left(\Omega_{\lambda}, P_{\lambda}\right)$ of probability spaces together with a family of measurable maps $f_{\lambda \mu}: \Omega_{\mu} \longrightarrow \Omega_{\lambda}$ defined for all $\lambda<\mu$, such that

1. $f_{\lambda \lambda}=\operatorname{Id}_{\Omega_{\lambda}}$,

2. $f_{\lambda \nu}=f_{\lambda \mu} \circ f_{\mu \nu}$ if $\lambda<\mu<\nu$,

3. $f_{\lambda \mu}\left(\Omega_{\mu}\right)=\Omega_{\lambda}$,

4. $\left(f_{\lambda \mu}\right)_{*} P_{\mu}=P_{\lambda}$.

The projective limit of such a system is by definition the set

$$
\lim _{\longleftarrow} \Omega_{\lambda}=\Omega=\left\{\left(\omega_{\lambda}\right)_{\lambda \in \Lambda} \in \prod_{\lambda \in \Lambda} \Omega_{\lambda} \mid \forall \lambda<\mu, f_{\lambda \mu}\left(\omega_{\mu}\right)=\omega_{\lambda}\right\} .
$$


For each $\lambda \in \Lambda$, the projection on the $\lambda$-th coordinate defines a map $f_{\lambda}: \Omega \longrightarrow \Omega_{\lambda}$. The main result is that, under certain conditions, there exist a $\sigma$-algebra and a probability measure on $\Omega$ which are consistent with all $P_{\lambda}$ via the maps $f_{\lambda}$.

Theorem 2.1.2 ( $\sigma$-algebra and a probability measure $P$ on $\Omega$ such that, for all $\lambda$ in $\Lambda, f_{\lambda_{*}} P=P_{\lambda}$. The space $(\Omega, P)$ is called projective limit of the family $\left(\Omega_{\lambda}, P_{\lambda} ; f_{\lambda \mu}\right)$.

It would be appealing to take the family of all graphs as index set $\Lambda$, with the order defined in 1.2.6. The problem is that given two graphs, it is not always true that there exists a third graph which is finer than both others, just because two edges belonging to two different graphs can intersect very badly. So, the first assumption about the ordering on $\Lambda$ would not be satisfied.

\subsection{Piecewise geodesic graphs}

We are led to consider a family of graphs small enough for that problem not to occur. A convenient family is that of graphs with edges piecewise geodesic for some Riemannian metric on $M$. Another possibility is to consider graphs with piecewise real analytic edges for some complex structure on $M$. This has been investigated by Ashtekar and Lewandowski [AD].

We fix a surface $(M, \sigma)$. For technical reasons, we suppose that $M$ is closed, until the section 2.8 where we shall derive the general case from the case without boundary.

Let us choose $q$ disjoint simple loops $L_{1}, \ldots, L_{q}$ on $M$ whose image is a smooth submanifold of $M$, in other words, $q$ disjoint embeddings of $S^{1}$ into $M$. We will sometimes think of these loops as the boundary of a submanifold of $M$ or just as loops along which we want to contition the holonomy.

Proposition 2.2.1 There exists a Riemannian metric on $M$ whose Riemannian volume coincides with $\sigma$ and such that $L_{1}, \ldots, L_{q}$ are geodesics.

Proof. If $q=0$, let us choose an arbitrary metric on $M$. Its Riemannian volume is equivalent to $\sigma$, with a smooth density. Multiplying the metric by an appropriate smooth positive function, we get a new metric, conformal to the first one, whose Riemannian volume is exactly $\sigma$.

If $q>0$, the proof is more complicated. Let us first construct a metric for which all $L_{i}$ 's are geodesic. Each $L_{i}$ has a tubular neighbourhood in $M$ which is diffeomorphic to a cylinder $S^{1} \times(-1,1) \ni(\theta, t)$, with $L_{i}=\{t=0\}$. In these coordinates, $L_{i}$ is certainly geodesic for the metric $d \theta^{2}+d t^{2}$. If the tubular neighbourhoods were chosen small enough to be disjoint, this procedure defines a Riemannian metric on the reunion of these cylinders and we extend it arbitrarily to a metric $g_{0}$ on the whole surface $M$. The loops $L_{1}, \ldots, L_{q}$ cut $M$ into submanifolds with boundary $M_{1}, \ldots, M_{k}$. Multiplying $g_{0}$ by a good positive function which is identically equal to 1 in a neighbourhood of each $L_{i}$, we can obtain a new metric $g_{1}$ for which all $L_{i}$ are still geodesics and also such that

$$
\operatorname{vol}_{g_{1}}\left(M_{i}\right)=\sigma\left(M_{i}\right) \quad \forall i=1 \ldots k
$$

Now we must redistribute the surface inside each $M_{i}$. We adapt a proof of Moser's theorem, which is for example proved in $B \mathrm{BG}$. 
Theorem 2.2.2 (Moser) Let $\alpha$ and $\beta$ be two volume 2 -forms on a closed compact surface $M$ such that

$$
\int_{M} \alpha=\int_{M} \beta
$$

There exists a diffeomorphism $\phi: M \longrightarrow M$ such that $\phi^{*} \beta=\alpha$.

We choose two forms $\alpha$ and $\beta$ representing respectively $\sigma$ and $\operatorname{vol}_{g_{1}}$. We know more about them than what is needed in Moser's theorem, but we also want to prove more: we would like to find a diffeomorphism of $M$ that sends $\alpha$ to $\beta$ and also that preserves the $L_{i}$ 's, so that they remain geodesic after pulling back $g_{1}$ by the diffeomorphism.

For each $i=1 \ldots q$, let $j_{i}: L_{i}([0,1]) \longrightarrow M$ denote the canonical injection. The fact that $\int_{M} \beta-\alpha=0$ implies that there exists a form $\gamma \in \Lambda^{1}\left(T^{*} M\right)$ such that $d \gamma=\beta-\alpha$. We show that $\gamma$ can be chosen such that $j_{i}^{*} \gamma=0$ for all $i$, or in other words such that $\gamma(X)=0$ for any vector $X$ tangent to a $L_{i}$. Pick $\gamma$ such that $d \gamma=\beta-\alpha$, consider the element $\left(\int_{L_{1}} \gamma, \ldots, \int_{L_{q}} \gamma\right)$ of $\mathbf{R}^{q}$ and the $q$-uple $\left(\left[L_{1}\right], \ldots,\left[L_{q}\right]\right)$ of vectors of $H_{1}(M ; \mathbf{Z})$. Suppose that $n_{1}, \ldots, n_{q}$ are integers such that $\sum_{i} n_{i}\left[L_{i}\right]=0$. Then $\sum_{i} n_{i}\left[L_{i}\right]$ is the boundary of a 2-chain in $M$, which is necessarily a linear combination of $M_{1}, \ldots, M_{k}$. Thanks to (2.1), this implies $\int_{\sum n_{i} L_{i}} \gamma=0=\sum_{i=1}^{q} n_{i} \int_{L_{i}} \gamma$. We proved that a relation $\sum n_{i}\left[L_{i}\right]=0$ implies $\sum n_{i} \int_{L_{i}} \gamma=0$. Thus there exists a linear form $\zeta$ on $H_{1}(M ; \mathbf{Z})$ such that $\left(\zeta,\left[L_{i}\right]\right)=\int_{L_{i}} \gamma$. This linear form can be represented by an element of $H^{1}(M ; \mathbf{R})$ and this element can be represented by a closed 1 -form on $M$ that we still denote by $\zeta$. The form $\gamma-\zeta$ satisfies:

$$
\left\{\begin{array}{l}
d(\gamma-\zeta)=d \gamma=\beta-\alpha \\
\int_{L_{i}} \gamma-\zeta=0 \quad \forall i=1 \ldots q
\end{array}\right.
$$

This last relation proves that for each $i, j_{i}^{*}(\gamma-\zeta)$ is exact on $L_{i}$ and can be written $d u_{i}$ with $u_{i} \in C^{\infty}\left(L_{i}\right)$. Let $u$ be a smooth function on $M$ such that $u_{\mid L_{i}}=u_{i}$ for each $i$. The form $\gamma-\zeta-d u$ satisfies:

$$
\left\{\begin{array}{l}
d(\gamma-\zeta-d u)=\beta-\alpha \\
j_{i}^{*}(\gamma-\zeta-d u)=0 \quad \forall i=1 \ldots q
\end{array}\right.
$$

We proved that it is possible to choose $\gamma$ such that $\gamma(X)=0$ for each vector $X$ tangent to a $L_{i}$ and we choose $\gamma$ in that way. The end of the proof is similar to that of Moser's theorem.

For each $t \in[0,1]$, set $\alpha_{t}=(1-t) \alpha+t \beta$ and define the vector field $X_{t}$ on $M$ by $i_{X_{t}} \alpha_{t}=-\gamma$. The field $X_{t}$ depends smoothly on $t$ and induces a flow $\left(\phi_{t}\right)_{t \in[0,1]}$. We compute the derivative of $\phi_{t}^{*} \alpha_{t}$. For any $t_{0} \in(0,1)$,

$$
\left.\frac{d}{d t}\right|_{t=t_{0}}\left(\phi_{t}^{*} \alpha_{t}\right)=\left.\frac{d}{d t}\right|_{t=t_{0}}\left(\phi_{t}^{*} \alpha_{t_{0}}\right)+\left.\frac{d}{d t}\right|_{t=t_{0}}\left(\phi_{t_{0}}^{*} \alpha_{t}\right)
$$

The second term of the r.h.s. is equal to

$$
\phi_{t_{0}}^{*}\left(\left.\frac{d}{d t}\right|_{t=t_{0}} \alpha_{t}\right)=\phi_{t_{0}}^{*}(\beta-\alpha) .
$$

We denote by $\mathcal{L}_{X_{t}}$ the Lie derivative with respect to the field $X_{t}$ and use Cartan's relation $\mathcal{L}_{X_{t}}=d \circ i_{X_{t}}+i_{X_{t}} \circ d$. We find that the first term is equal to

$$
\mathcal{L}_{X_{t_{0}}} \phi_{t_{0}}^{*} \alpha_{t_{0}}=d\left(i_{X_{t_{0}}} \phi_{t_{0}}^{*} \alpha_{t_{0}}\right)=d\left(\phi_{t_{0}}^{*} i_{X_{t_{0}}} \alpha_{t_{0}}\right)=-d\left(\phi_{t_{0}}^{*} \gamma\right)=-\phi_{t_{0}}^{*}(d \gamma)=-\phi_{t_{0}}^{*}(\beta-\alpha)
$$


Thus, $\frac{d}{d t} \phi_{t}^{*} \alpha_{t}=0$, so that $\phi_{1}^{*} \beta=\phi_{1}^{*} \alpha_{1}=\phi_{0}^{*} \alpha_{0}=\alpha$. For any vector $X$ tangent to a $L_{i}$, the equality $\alpha_{t}\left(X_{t}, X\right)=i_{X_{t}} \alpha_{t}(X)=\gamma(X)=0$ proves that the field $X_{t}$ is tangent to $L_{i}$. So the flow $\phi_{t}$ preserves the $L_{i}$ 's.

The Riemannian volume of the metric $g=\phi_{1}^{*} g_{1}$ is $\phi^{*} \operatorname{vol}_{g_{1}}=\sigma$. Moreover, $\phi_{1}$ is an isometry from $(M, g)$ into $\left(M, g_{1}\right)$ that preserves the $L_{i}$ 's. Since they are geodesics for $g_{1}$, they are also geodesics for $g$.

From now on, we fix on $M$ a metric given by the last proposition. Let us recall a classical result that summarizes the main property of the geodesics that we will use. A proof of a local version of this theorem can be found for example in $\mathrm{dC}$ (proposition 3.4.2). The compactness of $M$ allows to globalize the result.

Theorem 2.2.3 There exists a positive real number $R_{M}$, called convexity radius of $M$, such that if $x$ and $y$ are two points of $M$ contained in a ball of radius smaller than $R_{M}$, they are joined by a unique piece of minimizing geodesic and this piece of geodesic stays inside the ball.

This theorem implies in particular the following result:

Proposition 2.2.4 Let $\zeta_{1}$ and $\zeta_{2}$ be two finite pieces of geodesics. The intersection of $\zeta_{1}$ and $\zeta_{2}$ is the union of a finite number of isolated points and at most two segments.

Proof. If $\zeta_{1}$ and $\zeta_{2}$ meet at an infinity of points, it is easy to check that there exists a couple $\left(t_{1}, t_{2}\right)$ of times such that $\zeta_{1}\left(t_{1}\right)=\zeta_{2}\left(t_{2}\right)$ and $\dot{\zeta}_{1}\left(t_{1}\right)=\dot{\zeta}_{2}\left(t_{2}\right)$. So they are two pieces of the same infinite geodesic. If this geodesic is periodic, $\zeta_{1}$ and $\zeta_{2}$ can intersect along one or two segments. Otherwise, they have one segment in common plus a finite number of isolated points.

We denote by $\mathcal{G}$ the set of graphs whose edges are piecewise geodesic and such that $L_{1}, \ldots, L_{q}$ are in $\Gamma^{*}$. The set $\mathcal{G}$ is ordered by the relation $<$.

Proposition 2.2.5 Given two graphs $\Gamma_{1}, \Gamma_{2}$ in $\mathcal{G}$, there exists $\Gamma_{3}$ in $\mathcal{G}$ such that $\Gamma_{1}<\Gamma_{3}$ and $\Gamma_{2}<\Gamma_{3}$.

Proof. The idea is to superpose $\Gamma_{1}$ and $\Gamma_{2}$. Given an edge $a$ of $\Gamma_{2}$, we know that $a([0,1]) \cap \operatorname{Supp}\left(\Gamma_{1}\right)$ is a finite reunion of segments and points. So, it is possible to add a finite number of new vertices and new edges to $\Gamma_{1}$ in such a fashion that $a$ becomes a path in the new graph. Repeating this procedure for each edge of $\Gamma_{2}$ gives the result.

Let us fix an element $\left(x_{1}, \ldots, x_{q}\right)$ of $G^{q}$. With each graph $\Gamma \in \mathcal{G}$, we associated a space $\left(G^{\Gamma}, P^{\Gamma}\left(x_{1}, \ldots, x_{q}\right)\right)$. The last proposition states the last property that was missing for the family $\left(G^{\Gamma}, P^{\Gamma}\left(x_{1}, \ldots, x_{q}\right)\right), \Gamma \in \mathcal{G}$ to be a projective family of probability spaces. Each $G^{\Gamma}$ is compact, so theorem 2.1.2 asserts that this projetive family has a projective limit $\left(\Omega, \mathcal{A}, P\left(x_{1}, \ldots, x_{q}\right)\right)$ which is a probability space endowed with functions $f_{\Gamma}: \Omega \longrightarrow G^{\Gamma}$ such that $f_{\Gamma *} P\left(x_{1}, \ldots, x_{q}\right)=$ $P^{\Gamma}\left(x_{1}, \ldots, x_{q}\right)$. This space contains in itself the same information that is contained in all spaces $\left(G^{\Gamma}, P^{\Gamma}\left(x_{1}, \ldots, x_{q}\right)\right)$ : each random variable $h_{\zeta}: G^{\Gamma} \longrightarrow G$ gives rise to a random variable

$$
H_{\zeta}: \Omega \stackrel{f_{\Gamma}}{\longrightarrow} G^{\Gamma} \stackrel{h_{\zeta}}{\longrightarrow} G
$$


and the law of a $n$-uple $\left(h_{\zeta_{1}}, \ldots, h_{\zeta_{n}}\right)$ computed in any graph of $\mathcal{G}$ is always equal to that $\left(H_{\zeta_{1}}, \ldots, H_{\zeta_{n}}\right)$ under $P$. Moreover, since any piecewise geodesic path can be seen as a path in a graph, there is a well-defined random variable $H_{\zeta}$ on $\Omega$ associated with any such path $\zeta$. Remark that the multiplicativity property is preserved:

Proposition 2.2.6 Let $\zeta_{1}$ and $\zeta_{2}$ be two piecewise geodesic paths such that $\zeta_{1}(1)=\zeta_{2}(0)$. Then $H_{\zeta_{1} \zeta_{2}}=H_{\zeta_{2}} H_{\zeta_{1}}$ a.s.

From now on, we use greek letters to denote the piecewise geodesic paths and denote by $P G M$ the set of these paths.

\subsection{Preliminary results}

\subsubsection{Lassos}

Definition 2.3.1 A lasso is a simple loop or a path of the form $l=c b c^{-1}$, where $c$ is an injective path and $b$ a simple loop which meets $c$ only at its base point. The loop $b$ is detetermined by $l$ and is called the buckle of the lasso $l$.

A notion of lasso close to this one has already been used by Driver in [Dr2]. In [GKS], Gross, King and Sengupta also suggested that the use of lassos might be helpful in this construction.

Lassos are useful at least for two reasons: the first one is that it is easy to compute the law of their holonomy and the second one is that any reasonable loop can be decomposed in some sense into a product of lassos. Let us begin with this second point.

There is a natural equivalence relation between paths, which is the following:

Definition 2.3.2 Two paths are said to be basically equivalent if one of them can be written $c_{1} c_{2} c_{2}^{-1} c_{3}$ and the other one $c_{1} c_{3}$, where $c_{1}, c_{2}, c_{3} \in P M$. Two paths $c$ and $c^{\prime}$ are equivalent, and we denote $c \simeq c^{\prime}$, if there exists a finite chain $c=c_{0}, \ldots, c_{n}=c^{\prime}$ such that any two successive terms of this chain are basically equivalent.

Lemma 2.3.3 Let $\zeta_{1}$ and $\zeta_{2}$ be two paths of $P G M$ and suppose that $\zeta_{1} \simeq \zeta_{2}$. Then $H_{\zeta_{1}}=H_{\zeta_{2}}$ $P\left(x_{1}, \ldots, x_{q}\right)$-a.s.

Proof. This is a consequence of the multiplicativity of the random holonomy.

Let us define the class of paths that can be decomposed into a product of lassos.

Definition 2.3.4 A path $c \in P M$ is said to have finite self-intersection if there exists a graph $\Gamma$ such that $c \in \Gamma^{*}$.

Remark that this definition is not the usual one of finite self-intersection. Indeed, our definition allows for a path a finite number of points and also a finite number of segments as autointersection set. In particular, the proposition 2.2.4 shows that a piecewise geodesic path, which is a concatenation of injective pieces of different geodesics, has finite self-intersection in the sense of 2.3.4.

The Riemannian metric chosen on $M$ allows us to compute the length of a path $c$, that we denote by $\ell(c)$. 
Proposition 2.3.5 Let $c$ be a path with finite self-intersection.

1. If $c(0) \neq c(1)$ then $c$ is equivalent to a unique product $c \simeq l_{1} \ldots l_{p} c^{\prime}$, where the $l_{i}$ 's are lassos which are non equivalent to a constant loop and $c^{\prime}$ is an injective path joining $c(0)$ to $c(1)$. Moreover, if $b_{i}$ denotes the buckle of the lasso $l_{i}$ for each $i$, the following inequality holds:

$$
\ell(c) \geq \sum_{i=1}^{p} \ell\left(b_{i}\right)+\ell\left(c^{\prime}\right) .
$$

2. If $c(0)=c(1)$, the result remains true after removing $c^{\prime}$ of all expressions.

Proof. We proceed by induction on the number of edges in a decomposition of $c$ as a path in a graph. If $c$ is an edge, we are in the first case and the result is true. Suppose that $c=a_{1} \ldots a_{r}$. If $c$ is an injective path or a simple loop, the result is true. Otherwise, the idea is to trace $c$ out until the first time it intersects itself. Let $i$ be the smaller integer such that there exists $1 \leq j<i$ verifying $a_{j}(0)=a_{i}(1)$. Such an $i$ exists, and $(i, j) \neq(r, 1)$. We have:

$$
c \simeq a_{1} \ldots a_{j-1} \cdot a_{j} \ldots a_{i} \cdot\left(a_{1} \ldots a_{j-1}\right)^{-1} \cdot a_{1} \ldots a_{j-1} \cdot a_{i+1} \ldots a_{r} .
$$

It can happen that the first piece or the last piece of this decomposition are empty, respectively if $j=1$ or $i=r$, but these two situations cannot coexist. If $j=i-1$, it is possible that $a_{i}=a_{j}^{-1}$ so that $a_{j} a_{i}$ is equivalent to a constant path. This cannot happen if $j<i-1$, in which case $a_{j} \ldots a_{i}$ is a genuine simple path. Thus, the product of the three first terms is either equivalent to a constant path, or is a simple loop (if $j=1$ ), or a lasso. The product of the two last terms is the product of a number of edges which is positive and strictly less than $p$. So by induction, this path $\widetilde{c}$ is equivalent to $l_{1} \ldots l_{q} c^{\prime}$, or to $l_{1} \ldots l_{q}$ if $c$ is a loop. Note that $\ell(c)=\ell\left(a_{1}\right)+\ldots+\ell\left(a_{r}\right)=\ell(\widetilde{c})+\ell\left(a_{j}\right)+\ldots+\ell\left(a_{i}\right)$. So, by induction, $\ell(c) \geq \ell\left(b_{1}\right)+\ldots+\ell\left(b_{q}\right)+\ell\left(c^{\prime}\right)+\ell\left(a_{j}\right)+\ldots+\ell\left(a_{i}\right)$. In the case $j=i-1$ and $a_{j}=a_{i}^{-1}$, we have $c \simeq \widetilde{c}$ and the result is true with a strict inequality. Otherwise, there exists a lasso $l_{0}$ such that $c \simeq l_{0} \widetilde{c}$ and the length of the buckle of this lasso $l_{0}$ is exactly $\ell\left(a_{j}\right)+\ldots+\ell\left(a_{i}\right)$.

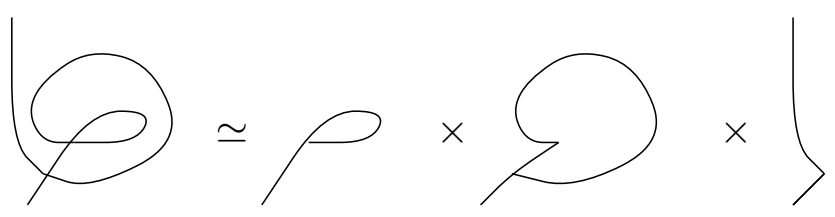

Figure 2.1: An example of decomposition.

\subsubsection{Holonomy along small piecewise geodesic loops}

In order to estimate the holonomy along a small lasso, we need, according to the proposition 1.8.5, to know the area enclosed by its buckle. This area can be controlled by the length of the buckle using an isoperimetric inequality. We recall a classical fact about open covering of metric compact sets. A proof can be found in $\mathrm{Ma}$.

Lemma 2.3.6 Let $M$ be a metric compact set. Let $\left(O_{i}\right)_{i \in I}$ be an open covering of $M$. There exists a positive real number $R$ called Lebesgue number of this covering, such that for any ball $B$ of radius smaller than $R$ in $M$, there exists an $i \in I$ such that $B \subset O_{i}$. 
Proposition 2.3.7 There exist $R>0$ and $K>0$ such that any simple loop $l$ contained in a ball $B$ of radius smaller than $R$ is the boundary of an open set $U \subset B$ such that

$$
\sigma(U) \leq K \ell(l)^{2}
$$

Proof. Let $R_{0}$ be such that any closed geodesic ball of $M$ of radius smaller than $R_{0}$ is diffeomorphic to a disk. Let $B_{1}, \ldots, B_{n}$ be a covering of $M$ by open balls of radius $R_{0}$. Let us denote by $g$ the metric on $M$ and $g_{0}$ the euclidean metric on $\mathbf{R}^{2}$. For each $i$, there exists a diffeomorphism $\phi_{i}: \overline{B_{i}} \longrightarrow \bar{D}(0,1) \subset \mathbf{R}^{2}$. Since the metrics $g$ and $\phi^{*} g_{0}$ can be compared on $\overline{B_{i}}$, the usual isoperimetric inequality on $\bar{D}(0,1)$ gives rise to an inequality on $\overline{B_{i}}$, with some constant $K_{i}$. Let $K$ be the supremum of $K_{1}, \ldots, K_{n}$. Let $R$ be a Lebesgue number of the covering $B_{1}, \ldots, B_{n}$. Then the statement holds with this choice of $K$ and $R$.

Now we can estimate the holonomy along a small lasso:

Proposition 2.3.8 There exist $L_{0}>0$ and $K>0$ such that if $\lambda$ is a piecewise geodesic lasso whose buckle $\beta$ has a length smaller than $L_{0}$, then

$$
E \rho\left(H_{\lambda}\right)=E d\left(H_{\lambda}, 1\right) \leq K \ell(\beta) .
$$

Proof. The lasso $\lambda$ can be written $\sigma \beta \sigma^{-1}$, so thanks to invariance by conjugation of the distance on $G$, we have $E \rho\left(H_{\lambda}\right)=E \rho\left(H_{\beta}\right)$. Let $L_{0}$ be shorter than the shortest length of a loop non homotopic to a point and also shorter than the radius $R$ given py the proposition 2.3.7. Then the hypothesis $\ell(\beta) \leq L_{0}$ implies that $\beta$ is the boundary of a small disk $D$. Using proposition 1.8.5, we get

$$
E \rho\left(H_{\beta}\right) \leq C \sqrt{\sigma(D)} \leq K \ell(\beta) .
$$

This result suggests that it will be possible to prove regularity results for the random holonomy using the following distance between $G$-valued random variables:

Definition 2.3.9 Let $X$ and $Y$ be two $G$-valued random variables defined on the same probability space. The distance $d_{P}(X, Y)$ is defined by:

$$
d_{P}(X, Y)=E d(X, Y),
$$

where $d$ is the biinvariant Riemannian distance on $G$.

The first example of such regularity results is the following one:

Proposition 2.3.10 Let $\zeta$ be a piecewise geodesic loop of length smaller than $L_{0}$. Then

$$
d_{P}\left(H_{\zeta}, 1\right) \leq K \ell(\zeta)
$$

Proof. Since $\zeta$ is piecewise geodesic, it has finite self-intersection. So it is equivalent to a product of piecewise geodesic lassos: $\zeta \simeq \lambda_{1} \ldots \lambda_{p}$. This gives:

$$
\begin{aligned}
d_{P}\left(H_{\zeta}, 1\right) & =d_{P}\left(H_{\lambda_{1}} \ldots H_{\lambda_{p}}, 1\right) \\
& \leq d_{P}\left(H_{\lambda_{1}} \ldots H_{\lambda_{p}}, H_{\lambda_{2}} \ldots H_{\lambda_{p}}\right)+\ldots+d_{P}\left(H_{\lambda_{p}}, 1\right) \\
& \leq d_{P}\left(H_{\lambda_{1}}, 1\right)+\ldots+d_{P}\left(H_{\lambda_{p}}, 1\right) \\
& \leq K\left(\ell\left(\beta_{1}\right)+\ldots \ell\left(\beta_{p}\right)\right) \\
& \leq K \ell(\zeta) .
\end{aligned}
$$




\subsubsection{Double layer potential of small piecewise geodesic loops}

Using the same techniques as in the preceding pararaph, we will estimate the double layer potential of a small loop. This is the first step in the proof of the proposition 2.6.8, that will play an important role in the study of the Abelian theory.

Recall that the definition of the potential (see 1.9.6) depends on a Riemannian metric on $M$ whose Riemannian volume is equal to $\sigma$. For the moment, we only know that the potential of any element of $P M$ is in $L^{2}(M, \sigma)$ (see theorem 1.9.11).

Proposition 2.3.11 Let $l$ be a lasso with buckle $b$. Suppose that $\ell(b) \leq L_{0}$, where $L_{0}$ is the length given by 2.3.8. Then

$$
\left\|u_{l}\right\|_{L^{2}} \leq K \ell(b)
$$

Proof. The length $L_{0}$ is such that $b$ is necessarily the boundary of a disk $D$ whose area satisfies $\sigma(D) \leq K \ell(b)^{2}$. Thus, by proposition 1.9.7,

$$
\left\|u_{b}\right\|_{L^{2}}=\left(\frac{\sigma(D) \sigma\left(D^{c}\right)}{\sigma(M)}\right)^{\frac{1}{2}} \leq \sigma(D)^{\frac{1}{2}} \leq K \ell(b) .
$$

Since $u_{l}=u_{b}$ a.e., we have the result.

As in the preceding paragraph, this result can be extended to loops with finite self-intersection.

Proposition 2.3.12 Let $l$ be a loop with finite self-intersection and of length smaller than $L_{0}$. Then

$$
\left\|u_{l}\right\|_{L^{2}} \leq K \ell(l)
$$

Proof. Let us write that $c$ is equivalent to a product of lassos: $c \simeq l_{1} \ldots l_{p}$. Two paths that are equivalent have the same double layer potential almost everywhere, so that

$$
\begin{aligned}
\left\|u_{c}\right\|_{L^{2}} & \leq\left\|u_{l_{1}}+\ldots+u_{l_{p}}\right\|_{L^{2}} \\
& \leq\left\|u_{l_{1}}\right\|_{L^{2}}+\ldots+\left\|u_{l_{p}}\right\|_{L^{2}} \\
& \leq K\left(\ell\left(b_{1}\right)+\ldots+\ell\left(b_{p}\right)\right) \\
& \leq K \ell(c) .
\end{aligned}
$$

The fact that the propositions 2.3.10 and 2.3.12 are very similar will allow us later, in proposition 2.5 .3 for example, to transpose directly some regularity results about the random variables $H$ to the double layer potential.

\subsubsection{Topology on the space of paths}

According to the proposition 1.8.5, it seems to be necessary to control the surface left between two loops in order to control the distance between their holonomies. Given a Riemannian metric on $M$, the uniform distance defined as follows:

$$
d_{\infty}\left(c_{1}, c_{2}\right)=\inf \sup _{t \in[0,1]} d\left(c_{1}(t), c_{2}(t)\right)
$$


allows to control this surface, where the infimum is taken over all reparametrizations of $c_{1}$ and $c_{2}$.

In the paper $[\overrightarrow{\mathrm{BE}}]$, C. Becker says that the double layer potential of a loop depends continuously of this loop in norm $L^{2}$ when the set of loops on $M$ is endowed with the topology induced by the uniform norm. His proposition 3.2 depends on the validity of this assertion, which is probably true if one restricts to simple loops, but not if one allows loops to have a self-intersection, even a finite one. Let us describe a counterexample. Becker stated his result on $\mathbf{R}^{2}$, but this does not change the situation very much. Let $M$ be the sphere $S^{2}$ embedded as usual in $\mathbf{R}^{3}$, endowed with the standard metric. Let us consider the pencil of planes $\left(P_{t}\right)_{t \in[0,4 \pi)}$ containing the horizontal line $z=0, y=-1$, indexed in the following way: denoting by $C_{t}$ the intersection of $S^{2}$ with the lower half-space bounded by $P_{t}$, we have $\sigma\left(C_{t}\right)=t$. For any $t \in[0,4 \pi)$, denote by $c_{t}$ a loop based at $(0,-1,0)$ whose image is the intersection of $P_{t}$ with $S^{2}$, oriented negatively with respect to the $z$ axis. Let $0<t_{1}<\ldots<t_{n}<4 \pi$ be $n$ distinct times and set $c=c_{t_{1}} \ldots c_{t_{n}}$. For each $i, u_{c_{i}}=\mathbf{1}_{C_{t_{i}}}-\frac{t_{i}}{4 \pi}$. Thus,

$$
\begin{aligned}
\left\|u_{c}\right\|_{L^{2}}^{2} & =\left\|\mathbf{1}_{C_{t_{1}}}+\ldots+\mathbf{1}_{C_{t_{n}}}\right\|_{L^{2}}^{2}+\frac{\left(t_{1}+\ldots+t_{n}\right)^{2}}{16 \pi^{2}} \\
& \quad-2\left(\frac{t_{1}+\ldots+t_{n}}{4 \pi}, \mathbf{1}_{C_{t_{1}}}+\ldots+\mathbf{1}_{C_{t_{n}}}\right)_{L^{2}} \\
& \geq n^{2} t_{1}+\frac{n^{2} t_{1}^{2}}{16 \pi^{2}}-\frac{n^{2} t_{n}^{2}}{2 \pi} .
\end{aligned}
$$

Suppose that $t_{1}=\frac{1}{n}$ and $t_{n}=\frac{2}{n}$. Then $\left\|u_{c}\right\|_{L^{2}}^{2}$ is of the order of $n$, so it grows to infinity when $n$ tends to infinity. But at the same time, the loop $c$ tends to the constant loop equal to $(0,-1,0)$ in the topology induced by the distance $d_{\infty}$. The potential of this constant loop being equal to zero, this contradicts the continuity.

Therefore, it is necessary to endow the space of paths with a topology finer than that induced by $d_{\infty}$ if we expect some kind of continuity of the double layer potential and of the random holonomy. It has emerged in the last paragraphs that the length plays a role in the continuity results.

Definition 2.3.13 On the set of paths PM, we define the distance $d_{1}$ by

$$
d_{1}\left(c_{1}, c_{2}\right)=d_{\infty}\left(c_{1}, c_{2}\right)+\left|\ell\left(c_{1}\right)-\ell\left(c_{2}\right)\right| .
$$

Proposition 2.3.14 The topology induced on PM by the distance $d_{1}$ does not depend on the Riemannian metric chosen on $M$.

Proof. By compactness of $M$, two different metrics induce two equivalent Riemannian distances on $M$ and thus two equivalent distances $d_{1}$ on $P M$.

\subsection{Approximation of embedded paths}

We want to extend the definition of the random holonomy to all paths in $P M$, by approximation. Since any path of $P M$ is, by definition, a concatenation of embedded submanifolds of $M$, it is natural to begin with those paths who are embedded submanifolds themselves. 


\subsubsection{Tubular neighbourhoods and Fermi coordinates}

These paths have the following nice property: they possess a tubular neighbourhood that can be described using Fermi coordinates. Let us fix a path $c$ which is an embedded submanifold. The proof of the following result can be found in [Gy]. Let us fix a parametrization of $c$ and a vector field $N$ along $c$, unitary and normal to $c$.

Proposition 2.4.1 There exists a positive real number $r$ such that the mapping

$$
\begin{aligned}
\psi:[0,1] \times[-r, r] & \longrightarrow M \\
(t, s) & \longmapsto \exp _{c(t)}\left(s N_{c(t)}\right)
\end{aligned}
$$

is a diffeomorphism onto its image, which is called tubular neighbourhood of $c$ or tube around $c$. The coordinates $(t, s)$ are called Fermi coordinates. They satisfy:

1. For any fixed $t_{0}$, the curve $s \mapsto \psi\left(t_{0}, s\right)$ is a piece of geodesic normal to $c$.

2. For any couple $(t, s) \in[0,1] \times[-r, r], d(\psi(t, s), c([0,1]))=s$.

We shall always assume that the radius of the tubular neighbourhoods that we consider are smaller than the convexity radius $R_{M}$ of $M$, defined in 2.2.3.

\subsubsection{Piecewise geodesic approximation}

The path $c$ is fixed until the end of the next section, together with a tubular neighbourhood of radius $r$.

Proposition 2.4.2 Let $x, y, z$ be three real numbers such that $0 \leq x<y<z \leq r$. There exists a piecewise geodesic path $\sigma$ such that

1. $\sigma(0)=\psi(0, y)$ and $\sigma(1)=\psi(1, y)$,

2. $\sigma((0,1)) \subset \psi((0,1) \times(x, z))$,

3. $\sigma$ is injective.

We construct $\sigma$ as an approximation of the path $c_{y}: t \mapsto \psi(t, y)$, in the same way as one would approximate a curve in $\mathbf{R}^{2}$ by piecewise linear paths.

Lemma 2.4.3 Set $\delta_{n}\left(c_{y}\right)=\sup _{0 \leq k \leq n-1} d\left(c_{y}\left(\frac{k}{n}\right), c_{y}\left(\frac{k+1}{n}\right)\right)$. Then

$$
\delta_{n}\left(c_{y}\right) \underset{n \rightarrow \infty}{\longrightarrow} 0 .
$$

Proof: The norm of the velocity of $c$ is bounded.

For $n$ large enough and for each $k=1 \ldots n$, the points $c_{y}\left(\frac{k-1}{n}\right)$ and $c_{y}\left(\frac{k}{n}\right)$ are close enough to be joined by a unique minimizing geodesic $\zeta_{n, k}$ that stays at a distance smaller than $\delta_{n}$ of $c_{y}\left(\frac{k}{n}\right)$. We will always assume that $n$ is large enough for this property to be true and set $\zeta_{n}=\zeta_{n, 1} \ldots \zeta_{n, n}$.

Lemma 2.4.4 For $n$ large enough, $\zeta_{n}$ is the graph of a continuous function in Fermi coordinates. More precisely, there exists a continuous function $\varphi:[0,1] \longrightarrow(-r, r)$ such that for each $t \in$ $[0,1], \zeta_{n}(t)=\psi(t, \varphi(t))$. 
Proof. It is sufficient to prove that each $\zeta_{n, k}$ is the graph of a continuous function defined on $\left[\frac{k-1}{n}, \frac{k}{n}\right]$ and that these functions can be put together to form $\varphi$. Let $n$ be such that $\delta_{n}<\frac{r-y}{2}$. We show that $\zeta_{n}$ stays inside the tubular neighbourhood of $c$.

The first point is that $\zeta_{n, k}$ cannot meet the horizontal boundary $\psi([0,1] \times\{-r, r\})$, because this boundary is at distance $r$ of $c$ and $\zeta_{n, k}$ stays at distance smaller than $\delta_{n}+y<r$.

The vertical part of the boundary $\psi(\{0,1\} \times[-r, r])$ is made of two pieces of minimizing geodesics, so that $\zeta_{n, k}$, which is also minimizing, cannot meet twice one of these pieces without belonging to the same infinite geodesic. This is impossible because the geodesics supporting the vertical boundary meet $c_{y}$ only once.

The only way $\zeta_{n, k}$ could exit the tube around $c$ would be to exit through one piece of the vertical boundary and get back through the other. Suppose that $n$ is large enough for $\delta_{n}$ being smaller than $\frac{1}{2} d(\psi(\{0\} \times[-r, r]), \psi(\{1\} \times[-r, r]))$. Then the situation described above cannot happen, since any two points of the image of $\zeta_{n, k}$ are at distance smaller than $\delta_{n}$. So, $\zeta_{n, k}$ stays inside the tube.

Each vertical slice $t=t_{0}$ of the tube is a minimizing piece of a geodesic that meets $c$ only once inside the tube, so that it meets $\zeta_{n, k}$ at most one time. Thus, $\zeta_{n, k}$ is the graph of a smooth function defined on the segment $\left[\frac{k-1}{n}, \frac{k}{n}\right]$, equal to $y$ at both end points of this segment. All these functions can be put together to make $\varphi$, which is continuous.

Proofof proposition 2.4.2. Choose $n$ be large enough for $\zeta_{n}$ to be the graph of a function in Fermi coordinates and such that $\delta_{n}<\inf (z-y, y-x)$. As a graph, $\zeta_{n}$ is necessary injective, which is statement 3. The inequality $|\varphi(t)-y|=d\left(\zeta_{n}(t), c_{y}\right) \leq \delta_{n}$ shows that $\zeta_{n}$ stays in $\psi([0,1] \times(x, z))$. Together with the fact that $\zeta_{n}$ meets at most once each vertical boundary, this gives statement 2. Statement 1 is a direct consequence of the definition of $\zeta_{n}$. So $\sigma=\zeta_{n}$ has all the properties required.

\subsection{Random holonomy along embedded paths}

We suppose that the surface of the tube is smaller than the constant $s$ given by the proposition 1.8.5. We prove that the random holonomy along a piecewise geodesic approximation of $c$ converges in probability to a random variable and that this limit does not depend on the particular choice of the approximation.

\subsubsection{Existence of a limit random holonomy}

For $n \geq 0$, set $x_{n}=\frac{r}{2^{n+1}}, y_{n}=\frac{3}{2} \frac{r}{2^{n+1}}, z_{n}=\frac{r}{2^{n}}$ and let $\sigma_{n}$ be a path given by the proposition 2.4.2. For each $n \geq 0$, let $\lambda_{n}$ denote the vertical segment joining $(0,0)$ to $\left(0, y_{n}\right)$ and $\rho_{n}$ the vertical segment joining $\left(1, y_{n}\right)$ to $(1,0)$. Finally, set $\alpha_{n}=\lambda_{n} \sigma_{n} \rho_{n}$.

Proposition 2.5.1 The sequence of random variables $\left(H_{\alpha_{n}}\right)_{n \geq 0}$ is a Cauchy sequence with respect to the distance $d_{P}$.

Proof. Let $m \geq n$ be two integers. We want to estimate $d_{P}\left(H_{\alpha_{m}}, H_{\alpha_{n}}\right)=d_{P}\left(H_{\alpha_{n}^{-1} \alpha_{m}}, 1\right)$. But $\alpha_{n}^{-1} \alpha_{m}$ is equivalent to a simple loop which is the boundary of an open set contained in 
$\psi\left([0,1] \times\left[0, \frac{r}{2^{n}}\right]\right)$. Thus, the assumption on the surface of the tube allows us to apply proposition 1.8.5. We get:

$$
d_{P}\left(H_{\alpha_{m}}, H_{\alpha_{n}}\right) \leq C \sigma\left(\psi\left([0,1] \times\left[0, \frac{r}{2^{n}}\right]\right)\right) \leq \frac{C^{\prime}}{2^{n}}
$$

This proves the result.

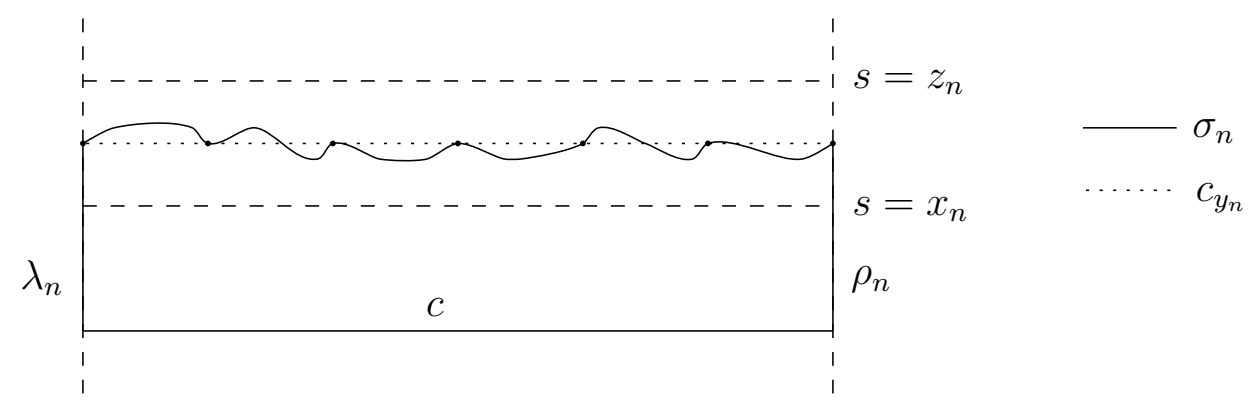

Figure 2.2: Definition of the sequence $\left(\alpha_{n}\right)$.

The space of $G$-valued random variables on $\left(\Omega, \mathcal{A}, P_{\beta}\right)$ endowed with the distance $d_{P}$ is complete: it can be isometrically embedded in a $L^{1}$ space by embedding $G$ isometrically in some $\mathbf{R}^{n}$. So the sequence $\left(H_{\alpha_{n}}\right)_{n \geq 0}$ has a limit that we denote by $H_{c}$, anticipating the fact that this limit does not depend on the choice of the sequence $\left(\alpha_{n}\right)$.

\subsubsection{Unicity of the limit random holonomy}

Lemma 2.5.2 For all $\varepsilon>0$, there exists $\delta>0$ such that if $\alpha$ is an injective piecewise geodesic path with the same end points as $c$, such that $\alpha((0,1)) \subset \psi((0,1) \times(-r, r))$ and such that $d_{\infty}(c, \alpha)<\delta$, then $d_{P}\left(H_{c}, H_{\alpha}\right)<\varepsilon$.

In this statement, it is not necessary to control $|\ell(\alpha)-\ell(c)|$ because $\alpha$ is assumed to be injective.

Proof. Let $C$ be the constant given by the proposition 1.8.5. Let $n$ be such that $d_{P}\left(H_{c}, H_{\alpha_{n}}\right)<$ $\varepsilon / 2$ and $C \sigma\left(\psi\left([0,1] \times\left[-\frac{r}{2^{n}}, \frac{r}{2^{n}}\right]\right)\right)<\varepsilon / 2$. Set $\delta=\frac{1}{2^{n+1}}$ and suppose that $d_{\infty}(c, \alpha)<\delta$. Then $\alpha$ meets $\alpha_{n}$ only at its end points. Thus $\alpha_{n} \alpha^{-1}$ is the boundary of an open set included in $\psi\left([0,1] \times\left[-\frac{r}{2^{n}}, \frac{r}{2^{n}}\right]\right)$, so that

$$
\begin{aligned}
d_{P}\left(H_{c}, H_{\alpha}\right) & \leq d_{P}\left(H_{c}, H_{\alpha_{n}}\right)+d_{P}\left(H_{\alpha_{n}}, H_{\alpha}\right) \\
& \leq \frac{\varepsilon}{2}+C \sigma\left(\psi\left([0,1] \times\left[-\frac{r}{2^{n}}, \frac{r}{2^{n}}\right]\right)\right) \\
& \leq \varepsilon .
\end{aligned}
$$

The control of the length of $\alpha$ allows to drop all restrictive conditions on $\alpha$, unless those concerning end points. The main result of this section is the following:

Proposition 2.5.3 For all $\varepsilon>0$, there exists $\delta>0$ such that if $\alpha$ is a piecewise geodesic path with the same end points as $c$ and such that $d_{1}(c, \alpha)<\delta$, then $d_{P}\left(H_{c}, H_{\alpha}\right)<\varepsilon$. 
Lemma 2.5.4 For all $\delta>0$, there exists $\eta>0$ such that if $c^{\prime}$ is another path such that $d_{\infty}\left(c, c^{\prime}\right)<\eta$, then $\ell\left(c^{\prime}\right)>\ell(c)-\delta$.

Note that this result is not symmetric in $c$ and $c^{\prime}$. Indeed, it is true that there exists $\eta^{\prime}$ such that $d_{\infty}\left(c, c^{\prime}\right)<\eta^{\prime}$ implies $\ell(c)>\ell\left(c^{\prime}\right)-\delta$, but $\eta^{\prime}$ may be much smaller than $\eta$ (consider for $c^{\prime}$ a zigzag approximating a straight line for example). One could reformulate this result by saying that for any sequence $c_{n}$ converging uniformly to $c, \lim \inf \ell\left(c_{n}\right) \geq \ell(c)$.

PROOF: Let $n$ be large enough for the following inequality to hold:

$$
\ell(c)-\sum_{k=0}^{n-1} d\left(c\left(\frac{k}{n}\right), c\left(\frac{k+1}{n}\right)\right)<\frac{\delta}{2} .
$$

Let $c^{\prime}$ be a path with fixed parametrization such that $d_{\infty}\left(c, c^{\prime}\right)<\frac{\delta}{4 n}$. Then, on one hand, $\ell\left(c^{\prime}\right) \geq \sum d\left(c^{\prime}\left(\frac{k}{n}\right), c^{\prime}\left(\frac{k+1}{n}\right)\right)$. On the other hand,

$$
\begin{aligned}
d\left(c\left(\frac{k}{n}\right), c(((k+1)) / n)\right) & \leq d\left(c\left(\frac{k}{n}\right), c^{\prime}\left(\frac{k}{n}\right)\right)+d\left(c^{\prime}\left(\frac{k}{n}\right), c^{\prime}\left(\frac{k+1}{n}\right)\right)+d\left(c^{\prime}\left(\frac{k+1}{n}\right), c\left(\frac{k+1}{n}\right)\right) \\
& \leq \frac{\delta}{2 n}+d\left(c^{\prime}\left(\frac{k}{n}\right), c^{\prime}\left(\frac{k+1}{n}\right)\right) .
\end{aligned}
$$

Thus, $\ell\left(c^{\prime}\right) \geq \sum_{k=0}^{n-1} d\left(c\left(\frac{k}{n}\right), c\left(\frac{k+1}{n}\right)\right)-\frac{\delta}{2} \geq \ell(c)-\delta$. We see that $\eta=\frac{\delta}{4 n}$ is a possible choice.

Proofof proposition 2.5.3. Denote by $\delta_{0}$ the distance between $c(0)$ and $c(1)$. Assume that $d_{\infty}(\sigma, c)$ is smaller than $\inf \left(r, \delta_{0} / 5\right)$. Recall that $r$ is assumed to be smaller than the convexity radius of $M$ (see 2.2.3).

The points $\alpha(0)$ and $\alpha(1)$ are respectively in the balls $B_{0}=B\left(c(0), 2 d_{\infty}(\alpha, c)\right)$ and $B_{1}=$ $B\left(c(1), 2 d_{\infty}(\alpha, c)\right)$. These balls are disjoint, hence there exists a last time $\tau_{0}$ at which $\alpha$ exits $B_{0}$ and a first time $\tau_{1}$ at which it enters $B_{1}$. The points $\alpha\left(\tau_{0}\right)$ and $\alpha\left(\tau_{1}\right)$ are necessarily inside the tube, for the points of $M$ at distance smaller than $r$ of $c$ are inside the tube or in $B_{0} \cup B_{1}$. In Fermi coordinates, we can write $\alpha\left(\tau_{0}\right)=\left(t_{0}, s_{0}\right)$ and $\alpha\left(\tau_{1}\right)=\left(t_{1}, s_{1}\right)$. Note that $t_{0}>0$ and $t_{1}<1$ : otherwise, we would have $\left|s_{0}\right|$ or $\left|s_{1}\right|$ equal to $2 d_{\infty}(\alpha, c)$.

Let $\gamma_{0}$ be the path that follows $c$ from time 0 to $t_{0}$ and then the geodesic normal to $c$ from $\left(t_{0}, 0\right)$ to $\left(t_{0}, s_{0}\right)$. Similarly, let $\gamma_{1}$ be the path that follows the normal geodesic from $\left(t_{1}, s_{1}\right)$ to $\left(t_{1}, 0\right)$ and then $c$ from time $t_{1}$ to 1 . We write $\alpha$ in the following way:

$$
\alpha \simeq \alpha_{\mid\left[0, \tau_{0}\right]} \gamma_{0}^{-1} \cdot \gamma_{0} \alpha_{\mid\left[\tau_{0}, \tau_{1}\right]} \gamma_{1} \cdot \gamma_{1}^{-1} \alpha_{\mid\left[\tau_{1}, 1\right]} \cdot
$$

The first and the third terms are small loops that we shall study later. Let us consider the central term $\gamma_{0} \alpha_{\mid\left[\tau_{0}, \tau_{1}\right]} \gamma_{1}$. It is contained in the tube around $c$ and has the same end points as $c$. Let us decompose it according to 2.3.5 into a product $\lambda_{1} \ldots \lambda_{p} \xi$, where the $\lambda_{i}$ 's are lassos based at $c(0)$ and $\xi$ is an injective path between $c(0)$ and $c(1)$. It is obvious that $d_{\infty}(c, \xi) \leq d_{\infty}(c, \alpha)$. This tells us, by proposition 2.5.2, that $H_{\xi}$ can be made arbitrarily close to $H_{c}$ by taking $d_{\infty}(c, \alpha)$ sufficiently small.

Let us fix a positive $\varepsilon$ and $\delta_{1}$ such that $d_{\infty}(c, \alpha)<\delta_{1}$ implies $d_{P}\left(H_{c}, H_{\xi}\right)<\varepsilon / 2$. It is enough now to control $d_{P}\left(H_{\xi}, H_{\alpha}\right)$.

$$
\begin{aligned}
d_{P}\left(H_{\xi}, H_{\alpha}\right) & =d_{P}\left(H_{\xi}, H_{\alpha_{\mid\left[0, \tau_{0}\right]} \gamma_{0}^{-1}} H_{\lambda_{1}} \ldots H_{\lambda_{p}} H_{\xi} H_{\gamma_{1}^{-1} \alpha_{\mid\left[\tau_{1}, 1\right]}}\right) \\
& \leq \sum_{i=1}^{p} d_{P}\left(H_{\lambda_{i}}, 1\right)+d_{P}\left(H_{\alpha_{\mid\left[0, \tau_{0}\right]} \gamma_{0}^{-1}}, 1\right)+d_{P}\left(H_{\gamma_{1}^{-1} \alpha_{\mid\left[\tau_{1}, 1\right]}}, 1\right) .
\end{aligned}
$$


We are led to consider the random variables associated with loops with finite self-intersection. According to 2.3.8, it it is sufficient to control their lengths. We already know by 2.5.4 that we can have $\ell(\xi) \geq \ell(c)-\varepsilon / 8$ provided $\delta_{1}$ and so $d_{\infty}(c, \xi)$ is small enough. If we impose now that $d_{1}(c, \alpha)<\delta_{1}$, instead of $d_{\infty}(c, \alpha)<\delta_{1}$, then we also get $\ell(\alpha)<\ell(c)+\varepsilon / 8$.

Then $0<\ell(\alpha)-\ell(\xi)<\varepsilon / 4$. Let us denote by $\beta_{1}, \ldots, \beta_{p}$ the buckles of the lassos $\lambda_{1}, \ldots, \lambda_{p}$. By 2.3.5,

$$
\ell(\xi)+\sum_{i} \ell\left(\beta_{i}\right) \leq \ell\left(\gamma_{0}\right)+\ell\left(\alpha_{\mid\left[\tau_{0}, \tau_{1}\right]}\right)+\ell\left(\gamma_{1}\right)
$$

and so

$$
\sum_{i} \ell\left(\beta_{i}\right)+\ell\left(\alpha_{\mid\left[0, \tau_{0}\right]} \gamma_{0}^{-1}\right)+\ell\left(\gamma_{1}^{-1} \alpha_{\mid\left[\tau_{1}, 1\right]}\right) \leq \frac{\varepsilon}{4}+2 \ell\left(\gamma_{0}\right)+2 \ell\left(\gamma_{1}\right)
$$

Since $\ell\left(\gamma_{i}\right) \leq 2 d_{\infty}(c, \alpha)+\ell\left(c([0,1]) \cap B_{i}\right)$, the lengths appearing in the right hand side can be made small by taking $d_{\infty}(c, \alpha)$ small enough. This is exactly what was needed to control $d_{P}\left(H_{\xi}, H_{\alpha}\right)$. This gives us a $\delta_{2}$ such that $d_{1}(c, \alpha)<\delta_{2}$ implies $d_{P}\left(H_{c}, H_{\alpha}\right)<\varepsilon$.

Corollary 2.5.5 Let $\left(\beta_{n}\right)_{n \geq 0}$ be any sequence of piecewise geodesic paths with the same end points as $c$ that converges to $c$. Then the sequence $\left(H_{\beta_{n}}\right)$ converges to $H_{c}$.

This proves that the variable $H_{c}$ does not depend on the particular choice of the sequence of paths approximating $c$.

\subsubsection{Continuity of the double layer potential (1)}

Following step by step the proofs of propositions 2.5.2 and 2.5.3 and replacing statements about random variables by statements about the double layer potential, according to the remark made at the end of paragraph 2.3.3, we get the following results:

Lemma 2.5.6 For all $\varepsilon>0$, there exists $\delta>0$ such that if $\alpha$ is an injective piecewise geodesic path with the same end points as $c$, such that $\alpha((0,1)) \subset \psi((0,1) \times(-r, r))$ and such that $d_{\infty}(c, \alpha)<\delta$, then $\left\|u_{\alpha}-u_{c}\right\|_{L^{2}}<\varepsilon$.

Proposition 2.5.7 For all $\varepsilon>0$, there exists $\delta>0$ such that if $\alpha$ is a piecewise geodesic path with the same end points as $c$ and such that $d_{1}(c, \alpha)<\delta$, then $\left\|u_{\alpha}-u_{c}\right\|_{L^{2}}<\varepsilon$.

\subsection{Random holonomy along arbitrary paths}

\subsubsection{Construction of the random holonomy}

Let $c$ be an element of $P M$. By definition, it can be written $c=c_{1} \ldots c_{p}$, where the $c_{i}$ 's are embedded paths, but this decomposition is far to be unique. Nevertheless, we prove that the random variable $H_{c_{p}} \ldots H_{c_{1}}$ depends only on $c$.

Lemma 2.6.1 Let $c$ be a path. There exists a sequence of piecewise geodesic paths with the same end points as $c$ that converges to $c$. 
Proof. Given a decomposition $c=c_{1} \ldots c_{p}$ of $c$ into a product of embedded paths, we concatenate sequences of paths that converge to each $c_{i}$ with fixed end points and get the required sequence.

Proposition 2.6.2 Let $\left(\alpha_{n}\right)$ be a sequence of piecewise geodesic paths that converges with fixed end points to $c$. The sequence $\left(H_{\alpha_{n}}\right)$ converges to the product $H_{c_{p}} \ldots H_{c_{1}}$.

The following corollary is in fact the main result of this paragraph.

Corollary 2.6.3 The product $H_{c_{p}} \ldots H_{c_{1}}$ is independent of the choice of the decomposition of $c$ and it is equal to the common limit of all sequences $\left(H_{\alpha_{n}}\right)$ associated with sequences $\left(\alpha_{n}\right)$ of piecewise geodesic paths converging to $c$ with fixed end points. We shall denote it by $H_{c}$.

Proofof proposition 2.6.2. We cut $\alpha_{n}$ in a way that corresonds to the decomposition of $c$. Let us fix a parametrization of $c$ such that $c_{i}=c_{\mid\left[\frac{i-1}{p}, \frac{i}{p}\right]}$. Let us also fix a parametrization of each $\alpha_{n}$ such that the uniform convergence $d\left(c, \alpha_{n}\right) \rightarrow 0$ holds with these parametrizations. Set $\alpha_{i, n}=\alpha_{n \mid\left[\frac{i-1}{p}, \frac{i}{p}\right]}$. Let us show that for each $i, \alpha_{i, n} \underset{n \rightarrow \infty}{\longrightarrow} c_{i}$.

The first point is that $d_{\infty}\left(c_{i}, \alpha_{i, n}\right) \leq d_{\infty}\left(c, \alpha_{n}\right) \underset{n \rightarrow \infty}{\longrightarrow} 0$, parametrizations being fixed. Now let us choose $\varepsilon>0$ and $n$ large enough such that for all $i=1, \ldots, p, \ell\left(\alpha_{i, n}\right) \geq \ell\left(c_{i}\right)-\frac{\varepsilon}{p}$ (using lemma 2.5.4) and $\ell\left(\alpha_{n}\right) \leq \ell(c)+\varepsilon$. Then

$$
\begin{aligned}
-\frac{\varepsilon}{p} \leq \ell\left(\alpha_{i, n}\right)-\ell\left(c_{i}\right) & =\ell\left(\alpha_{n}\right)-\ell(c)-\sum_{j \neq i}\left(\ell\left(\alpha_{j ; n}\right)-\ell\left(c_{j}\right)\right) \\
& \leq 2 \varepsilon .
\end{aligned}
$$

So we also have $\ell\left(\alpha_{i, n}\right) \underset{n \rightarrow \infty}{\longrightarrow} \ell\left(c_{i}\right)$ for all $i$. Consider now for each $i$ and each $n$ the path $\widetilde{\alpha}_{i, n}$ which is $\alpha_{i, n}$ concatenated at both end points with a minimizing piece of geodesic in order to have the same end points as $c_{i}$. If $n$ is large enough, $\alpha_{n}$ is close enough to $c$ for these pieces of minimizing geodesic to be uniquely defined. So, these geodesic segments cancel out in the product $\widetilde{\alpha}_{1, n} \ldots \widetilde{\alpha}_{p, n}$ which is equivalent to $\alpha_{1, n} \ldots \alpha_{p, n}$. On the other hand, we have $\widetilde{\alpha}_{i, n} \stackrel{d_{1}}{\longrightarrow} c_{i}$. Indeed, the small geodesic pieces stay close to each $c_{i}$ and their length converges to zero. Thus, the corollary 2.5.5 implies $H_{\widetilde{\alpha}_{i, n}} \underset{n \rightarrow \infty}{\longrightarrow} H_{c_{i}}$ which gives the result:

$$
H_{\alpha_{n}}=H_{\widetilde{\alpha}_{p ; n}} \ldots H_{\widetilde{\alpha}_{1 ; n}} \underset{n \rightarrow \infty}{\longrightarrow} H_{c_{p}} \ldots H_{c_{1}}
$$

\subsubsection{Continuity of the random holonomy}

At this point, we constructed a random holonomy along each path $c$ of $P M$. This random holonomy is a $G$-valued random variable on the probability space $\left(\Omega, \mathcal{A}, P\left(x_{1}, \ldots, x_{q}\right)\right)$. Let us state some of its basic properties.

Proposition 2.6.4 Let $c, c_{1}, c_{2}$ be elements of $P M$.

1. $H_{c^{-1}}=H_{c}^{-1}$ a.s.

2. The random variable $H_{c}$ depends only on the equivalence class of $c$ for the relation $\simeq$.

3. If $c_{1}$ and $c_{2}$ satisfy $c_{1}(1)=c_{2}(0)$ then $H_{c_{1} c_{2}}=H_{c_{2}} H_{c_{1}}$ a.s. 
Proof: Property (3) is obvious by putting together two decompositions of $c_{1}$ and $c_{2}$ and the property (2) is a direct consequence of (1). To prove (1), just note that this is true for piecewise geodesic paths by construction, and that if $\alpha_{n} \underset{n \rightarrow \infty}{\stackrel{d_{1}}{\rightarrow}} c$, then $\alpha_{n}^{-1} \underset{n \rightarrow \infty}{\stackrel{d_{1}}{\longrightarrow}} c^{-1}$.

We still have to prove that the law of this random holonomy does not depend on choice of the Riemannian metric used in the construction. For this, we need a regularity property which is the object of the next proposition.

Proposition 2.6.5 Let $c$ be a path of $P M$. For any $\varepsilon>0$, there exists $\delta>0$ such that if $c^{\prime}$ is another path of $P M$ with the same end points as $c$ and if $d_{1}\left(c, c^{\prime}\right)<\delta$, then $d_{P}\left(H_{c}, H_{c^{\prime}}\right)<\varepsilon$.

Proof. Let $\delta_{0}>0$ be given by the proposition 2.5.3 such that for any piecewise geodesic path $\alpha$ with the same end points as $c, d_{1}(c, \alpha)<\delta_{0}$, implies $d_{P}\left(H_{c}, H_{\alpha}\right)<\varepsilon$. Let $\delta=\frac{\delta_{0}}{2}$. Suppose that $c^{\prime}$ is a path of $P M$ with the same end points as $c$ such that $d_{1}\left(c, c^{\prime}\right)<\delta$. Let $\alpha$ be a piecewise geodesic path such that, simultaneously, $d_{1}\left(\alpha, c^{\prime}\right)<\delta$ and $d_{P}\left(H_{c^{\prime}}, H_{\alpha}\right)<\frac{\varepsilon}{2}$. Then $d_{1}(c, \alpha)<\delta_{0}$, so that

$$
d_{P}\left(H_{c}, H_{c^{\prime}}\right) \leq d_{P}\left(H_{c}, H_{\alpha}\right)+d_{P}\left(H_{\alpha}, H_{c^{\prime}}\right)<\varepsilon
$$

Let us state a result that summarizes the results of the procedure of piecewise geodesic approximation. It is in fact the center of the continuum limit procedure. We put together the propositions 2.6.2, 2.6.3 and 2.6.5.

Proposition 2.6.6 Let $c$ be a path of PM. For any sequence $\left(\alpha_{n}\right)_{n \geq 0}$ of piecewise geodesic paths converging to $c$ with fixed end points, the sequence $\left(H_{\alpha_{n}}\right)_{n \geq 0}$ converges to a random variable that depends only on $c$ and that we denote by $H_{c}$. Moreover, for any $\varepsilon>0$, there exists $\delta>0$ such that if $c^{\prime}$ is another path of $P M$ with the same end points as $c$ and if $d_{1}\left(c, c^{\prime}\right)<\delta$ then $d_{P}\left(H_{c}, H_{c^{\prime}}\right)<\varepsilon$.

\subsubsection{Continuity of the double layer potential (2)}

One more time, we transpose directly the preceding arguments to the double layer potential and get the following result:

Proposition 2.6.7 Let $c$ be a path of $P M$. For any $\varepsilon>0$, there exists $\delta>0$ such that if $c^{\prime}$ is another path of PM with the same end points as $c$ and if $d_{1}\left(c, c^{\prime}\right)<\delta$, then $\left\|u_{c}-u_{c^{\prime}}\right\|_{L^{2}}<\varepsilon$.

Corollary 2.6.8 Let $l$ be a loop of LM. For any $\varepsilon>0$, there exists $\delta>0$ such that if $l^{\prime}$ is another loop of $L M$ and if $d_{1}\left(l, l^{\prime}\right)<\delta$, then $\left\|u_{l}-u_{l^{\prime}}\right\|_{L^{2}}<\varepsilon$.

Proof. Let $\delta_{0}$ be given by the preceding proposition and set $\delta=\frac{\delta_{0}}{3}$. Let $l^{\prime} \in L M$ be such that $d_{1}\left(l, l^{\prime}\right)<\delta$. We have in particular $d\left(l(0), l^{\prime}(0)\right)<\delta$. Let $\sigma$ be a minimizing geodesic from $l(0)$ to $l^{\prime}(0)$. Then $\widetilde{l}^{\prime}=\sigma l^{\prime} \sigma^{-1}$ satisfies $u_{l^{\prime}}=u_{l^{\prime}}$ a.e. and $d_{1}\left(\widetilde{l^{\prime}}, l\right)<\delta_{0}$. Moreover, $\widetilde{l}^{\prime}$ has the same end points as $l$. Thus,

$$
\left\|u_{l}-u_{l^{\prime}}\right\|_{L^{2}}=\left\|u_{l}-u_{\widetilde{l}^{\prime}}\right\|_{L^{2}}<\varepsilon
$$




\subsection{Law of the random holonomy}

For the moment, we are only able to write down the law of the holonomy along piecewise geodesic paths. We want to show that the law of the holonomy along arbitrary families of paths with finite self-intersection is what we expect it to be, namely that given by the discrete theory. The goal of this section is to prove the following proposition:

Proposition 2.7.1 Let $\Gamma=\left\{a_{1}, \ldots, a_{r}\right\}$ be a graph on $M$ such that $L_{1}, \ldots L_{q} \in \Gamma^{*}$. For any function $f$ continuous on $G^{\Gamma}$, we have:

$$
\operatorname{Ef}\left(H_{a_{1}}, \ldots, H_{a_{r}}\right)=\int_{G^{\Gamma}} f d P_{\beta}^{\Gamma} .
$$

A very important consequence of this result is the independence of the construction with respect to the Riemannian metric:

Corollary 2.7.2 The law of the family $\left(H_{c}\right)_{c \in P M}$ does not depend on the choice of the Riemannian metric that was used throughout the construction.

Proof. Consider two families of variables obtained with two different choices of metric. By the preceding proposition, these families have the same law on the set of paths that are piecewise geodesic for, say, the first metric. By proposition 2.6.5, both families are continuous in a sense that is strong enough to guarantee that their laws coincide on the whole set $P M$.

In order to prove the proposition 2.7.1, we need a technical result about the approximation of graphs by piecewise geodesic graphs. Before to state this result, let us make some remarks about the edges and faces in a graph in $M$.

Recall that a path and hence an edge must by definition have non-zero derivatives at its end points. This avoids pathological behaviours. For example, consider all edges that share a given vertex of a graph and a small geodesic circle centered at this vertex. If the radius of this circle is small enough, each edge cuts it only once, and the order of the intersection points, which does not depend on the radius of the circle, defines a cyclic order on the set of these edges.

Now, consider two edges that are adjacent for this order. They bound at least one common face. Thus, if $M$ is oriented, a couple of adjacent edges determines a face of the graph (see fig. 2.3). Conversely, given a face, any two consecutive edges of the boundary of this face are adjacent at the vertex that they share, or eventually at both vertices if they share two.

Proposition 2.7.3 Let $\Gamma=\left\{a_{1}, \ldots, a_{r}\right\}$ be a graph such that $L_{1}, \ldots, L_{q} \in \Gamma^{*}$. For any $\varepsilon>0$, there exists a graph $\Gamma_{\varepsilon}=\left\{\alpha_{1}, \ldots, \alpha_{r}\right\}$ with piecewise geodesic edges such that:

1. $\Gamma_{\varepsilon}$ and $\Gamma$ have the same vertices,

2. For each $i=1, \ldots, r, d_{1}\left(\alpha_{i}, a_{i}\right)<\varepsilon$,

3. $L_{1}, \ldots, L_{q} \in \Gamma_{\varepsilon}^{*}$

4. For each $i=1, \ldots, r, a_{i}$ and $\alpha_{i}$ are in the same connected component of the complementary of the unions of the images of the $L_{i}$ 's.

Let us denote by $\alpha: \Gamma^{*} \longrightarrow \Gamma_{\varepsilon}^{*}$ the multiplicative map that sends $a_{i}$ to $\alpha_{i}$. It is possible to construct $\Gamma_{\varepsilon}$ in such a way that this map induces a one-to-one correspondence still denoted by $\alpha: \mathcal{F}(\Gamma) \longrightarrow \mathcal{F}\left(\Gamma_{\varepsilon}\right)$ such that $\partial \alpha(F)=\alpha(\partial F)$ and $\sigma(F-\partial F)<\varepsilon$, where - denotes the symmetric difference. 


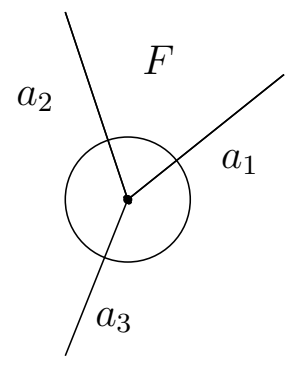

Figure 2.3: The face determined by two adjacent edges.

Proof. The property 4 is a consequence of 2 and 3 . Indeed, if $a_{i}$ is in a given connected component, $\alpha_{i}$ meets this component if $\varepsilon$ is small enough, by 2. But $\alpha_{i}$ could only exit this component by crossing $L_{i}$ at a point which is not an end point of $\alpha_{i}$, which is impossible by 3 and by the definition of graphs.

Let $\mathcal{V}(\Gamma)=\left\{s_{1}, \ldots, s_{p}\right\}$ denote the set of vertices of $\Gamma$. Let $r$ be a positive real number that "localizes the vertices of $\Gamma$ ", i.e. small enough to satisfy the following properties:

1. The balls $B\left(s_{i}, r\right)$ are pairwise disjoint.

2. For every pair $\left(a_{i}, s_{j}\right)$ with $a_{i} \in \Gamma$ and $s_{j}$ an end point of $a_{i}, a_{i}$ meets only once and transversally any circle centered at $s_{j}$ and of radius smaller than $r$. Moreover, the length of the portion of $a_{i}$ in the corresponding ball is smaller than $\varepsilon / 16$.

3. For any pair $\left(a_{i}, s_{j}\right)$ where $s_{j}$ is not an end point of $a_{i}, a_{i}$ does not meet the ball $B\left(s_{j}, r\right)$.

4. The sum of the surfaces of the ball $B\left(s_{i}, r\right)$ is smaller than $\varepsilon / 2$.

5. $r<\varepsilon / 16$ and $r<R_{M}$, where $R_{M}$ is the convexity radius of $M$.

All properties remain true for $r^{\prime}<r$ once they are true for $r$, so that it is not a problem to get them simultaneously.

Let $t$ be a positive real number such that $\sigma(\{d(\cdot, \Gamma)<t\})<\frac{\varepsilon}{2}$. Let $\widehat{a}_{i}$ denote the portion of $a_{i}$ outside the disks of radius $r$ around its end points. Let $\delta$ be the smallest distance between the images of two distinct $\widehat{a}_{i}$. For each $i$, let $\gamma_{i}$ be an injective piecewise geodesic path with the same end points as $\widehat{a}_{i}$, such that $d_{1}\left(\gamma_{i}, \widehat{a}_{i}\right)<\inf (\varepsilon / 4, \delta / 2, t)$ and that never meets the balls $B\left(s_{j}, r\right)$, except at its ends. This last condition can be obtained because $a_{i}$ cuts $B\left(s_{j}, r\right)$ transversally: in a neighbourhood of each end point of $\widehat{a}_{i}$, there is a half-tube around $\widehat{a}_{i}$ that does not meet $B\left(s_{j}, r\right)$. It is possible around each end point of $\widehat{a}_{i}$ to construct $\gamma_{i}$ inside this half-tube. By definition of $\delta$, the $\gamma_{i}$ 's are disjoint.

Now define $\alpha_{i}$ for each $i$ such that $a_{i}$ is not piecewise geodesic as the concatenation of the minimizing geodesic from $a_{i}(0)$ to $\widehat{a}_{i}(0)$, of $\gamma_{i}$ and of the minimizing geodesic from $\widehat{a}_{i}(1)$ to $a_{i}(1)$ (see fig. 2.4). Assumption 5 ensures that these minimizing geodesics are well defined. Assumptions 2 and 5 imply that $d_{1}\left(\alpha_{i}, a_{i}\right)<\varepsilon$. The edges of the decompositions of the $L_{i}$ 's are already piecewise geodesic. Hence we only rename them, setting $\alpha_{i}=a_{i}$.

The $\alpha_{i}$ 's are edges. Moreover, they were constructed in such a way that they meet only at their ends: we already noticed that they do not meet outside the balls around the vertices of $\Gamma$, and they cannot meet more than once inside these balls according to the local properties of geodesics. Thus, the graph $\Gamma_{\varepsilon}=\left\{\alpha_{1}, \ldots, \alpha_{r}\right\}$ exists and has the same vertices as $\Gamma$.

We just proved that properties 1 and 2. Property 3 is true because we kept the edges corresponding to the $L_{i}$ 's and property 4 follows, according to the remark made at the beginning 


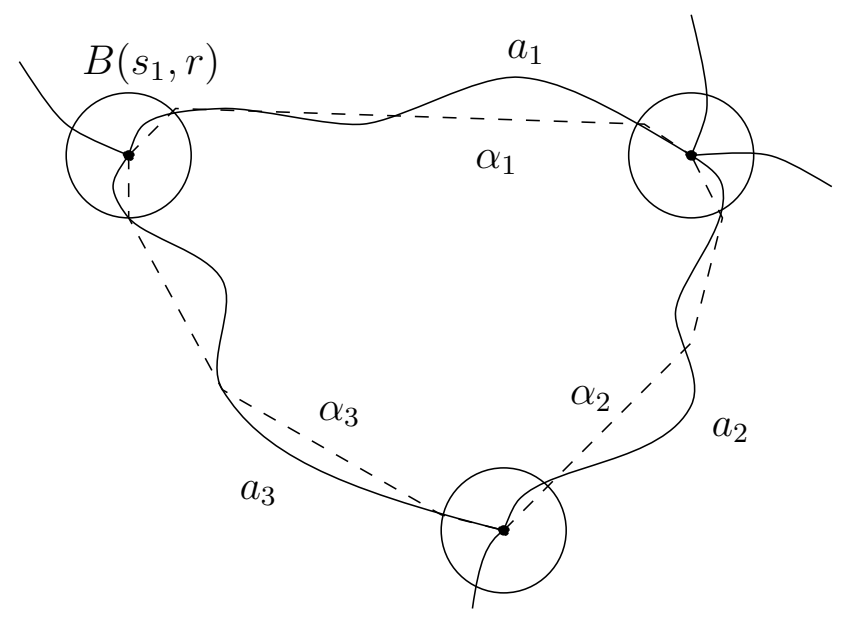

Figure 2.4: Definition of the edges of the graph $\Gamma_{\varepsilon}$.

of the proof. It remains to prove the last part of the statement.

Consider edges of $\Gamma$ that share a given vertex. They are given a cyclic order. By definition, the corresponding $\alpha_{i}$ 's cut the circle of radius $r$ around this vertex in the same order, so that the multiplicative application $\alpha: \Gamma^{*} \rightarrow \Gamma_{g}^{*}$ defined by $\alpha\left(a_{i}\right)=\alpha_{i}$ preserves the cyclic order at each vertex.

Given a pair of edges of $\Gamma$ that determine the face $F$, the pair of corresponding edges of $\Gamma_{\varepsilon}$ is a pair of adjacent edges that determine a face of $\Gamma_{\varepsilon}$. This face does not depend on the particular choice of the edges that represent $F$ and we denote it by $\alpha(F)$. By construction, we have the relation $\partial(\alpha(F))=\alpha(\partial F)$.

The symmetric difference of $F$ and $\alpha(F)$ is contained in the reunion of the balls $B\left(s_{j}, r\right)$ and the sets $\left\{d\left(\cdot, \widehat{a}_{i}\right)<t\right\}$. By assumption 4$)$ and by definition of $t$, we know that the total volume of these sets is smaller than $\varepsilon$. Thus, $\operatorname{vol}(F-\partial F)<\varepsilon$. Moreover, this inequality characterizes $\alpha(F)$ among the faces of $\Gamma_{\varepsilon}$ that have $\alpha(\partial F)$ as boundary, if there is more than one, provided $\sigma(M)$ is greater than $2 \varepsilon$.

Proof OF 2.7.1. For each integer $n$, the preceding proposition gives a graph $\Gamma_{\frac{1}{n}}=$ $\left\{\alpha_{1, n}, \ldots, \alpha_{r, n}\right\}$. For each $i=1, \ldots, r$, the sequence $\left(\alpha_{i, n}\right)$ converges to $a_{i}$ with fixed end points, so that $H_{\alpha_{i, n}} \longrightarrow H_{a_{i}}$. In particular, we have the convergence in law:

$$
\left(H_{\alpha_{1, n}}, \ldots, H_{\alpha_{r, n}}\right) \underset{n \rightarrow \infty}{\stackrel{\text { law }}{\longrightarrow}}\left(H_{a_{1}}, \ldots, H_{a_{r}}\right) .
$$

Thus, for any function $f$ continuous on $G^{\Gamma}$,

$$
\begin{aligned}
E f\left(H_{a_{1}}, \ldots, H_{a_{r}}\right) & =\lim _{n \rightarrow \infty} \frac{1}{Z^{\Gamma} \frac{1}{n}} \int_{G^{\Gamma}} f\left(g_{1}, \ldots, g_{r}\right) \prod_{F \in \mathcal{F}\left(\Gamma_{\frac{1}{n}}\right)} p_{\sigma(F)}\left(h_{\partial F}\right) \\
& =\left(\lim _{n \rightarrow \infty} \frac{1}{Z^{\Gamma^{\frac{1}{n}}}}\right) \int_{G^{\Gamma}} f\left(g_{1}, \ldots, g_{r}\right) \prod_{F \in \mathcal{F}(\Gamma)} p_{\sigma(F)}\left(h_{\partial F}\right)
\end{aligned}
$$




$$
d \nu_{x_{1}} \ldots d \nu_{x_{q}} d g^{\prime}
$$

using the fact that $\sigma(\alpha(F))$ tends to $\sigma(F)$ when $n$ tends to infinifty.

Recall from proposition 1.6.5 that the conditional partition functions computed in two graphs, one being finer than the other, are equal. But given two piecewise geodesic graphs, there exists a third one which is finer than both others, as was proved in 2.2.5. Thus the partition function is the same for all piecewise geodesic graphs, and the sequence $\left(Z^{\Gamma} \frac{1}{n}\right)$ is constant. Its value can be computed by setting $f$ identically equal to 1 : we find that it is equal to $Z^{\Gamma}$. This proves the result.

By the way, we proved the following important result:

Proposition 2.7.4 Let $\Gamma$ be a graph such that $L_{1}, \ldots, L_{q} \in \Gamma^{*}$. Then the value of the conditional partition function $Z^{\Gamma}\left(x_{1}, \ldots, x_{q}\right)$ does not depend on $\Gamma$.

\subsection{Surfaces with boundary}

At the beginning of this chapter, we restricted ourselves for technical reasons to surfaces without boundary. In this section, we will extend the construction of the random holonomy to the case of surfaces with boundary.

\subsubsection{Natural law of the holonomy along the boundary}

Let $(M, \sigma)$ be a surface with a boundary $\partial M=N_{1} \cup \ldots \cup N_{p}$. In order to construct the holonomy along the paths of $M$, we shall embed $M$ in a minimal closure and use the construction described in the preceding sections. But if we want this procedure to give a result independent of the closure of $M$, and we do, it is necessary to condition the holonomy along every component of $\partial M$. If this was not our first intention, say if we expected only to impose the holonomy along $N_{1}$, to be equal to $x$ for example, we need to know the natural law of the holonomy along the whole boundary under $P(x)$. Then, we will artificially impose this natural law when working on the closure of $M$. We begin by defining this natural law.

Let $L_{1}, \ldots, L_{q}$ be disjoint simple loops on $M$ whose image is included in the interior of $M$. Let $N_{1}, \ldots, N_{k}$ be the components of $\partial M$ along which we want to impose the holonomy. Let $x_{1}, \ldots, x_{k}, y_{1}, \ldots, y_{q}$ be elements of $G$. The $k$ first elements correspond to the components of $\partial M$, the $q$ others to the interior loops.

Proposition 2.8.1 Let $\Gamma$ be a graph on $M$ such that $L_{1}, \ldots, L_{q} \in \Gamma^{*}$. The law of the random variable $\left(h_{N_{1}}, \ldots, h_{N_{p}}, h_{L_{1}}, \ldots, h_{L_{q}}\right)$ defined on the probability space $\left(G^{\Gamma}, P\left(x_{1}, \ldots, x_{k}, y_{1}, \ldots, y_{q}\right)\right)$ does not depend on $\Gamma$. We will denote it by $\beta\left(x_{1}, \ldots, x_{k}, y_{1}, \ldots, y_{q}\right)$.

Lemma 2.8.2 The propositions 2.7.3 and 2.7.4 hold on surfaces with boundary.

Proof. Note that 2.7.3 implies 2.7.4, using the computation done at the end of its proof. Thus, it is sufficient to prove that 2.7 .3 holds. For this, embed $M$ in a minimal closure $M_{1}$. A graph $\Gamma$ on $M$ induces a graph on $M_{1}$, which can be approximated by piecewise geodesic graphs. If each component of $\partial M$ is the image of one of the $L_{i}$ 's, then the property (4) says exactly that 
the approximating graphs stay inside $M$.

Proofof proposition 2.8.1. Let us endow $M$ with a Riemannian metric for which $N_{1}, \ldots, N_{p}$, $L_{1}, \ldots, L_{q}$ are geodesics. The law of $\left(h_{N_{1}}, \ldots, h_{N_{p}}, h_{L_{1}}, \ldots, h_{L_{q}}\right)$ does not depend on $\Gamma$ provided it is piecewise geodesic, by invariance by subdivision.

Now consider an arbitrary graph $\Gamma$. According to the preceding lemma, we can approximate it by piecewise geodesic graphs, for which the law we are interested in is always the same. The convergence of the joint law of the holonomy along all edges proves the result.

The lemma 1.5.2 gives us an expression of $\beta\left(x_{1}, \ldots, x_{k}, y_{1}, \ldots, y_{q}\right)$. We state it here again.

Lemma 2.8.3 The following equality between measures on $G^{p+q}$ holds:

$$
\begin{aligned}
& \beta\left(x_{1}, \ldots, x_{k}, y_{1}, \ldots, y_{q}\right)= \\
& \quad \delta_{\left(x_{1}, \ldots, x_{k}\right)} \otimes \frac{Z\left(x_{1}, \ldots, x_{k}, x_{k+1}^{\prime}, \ldots, x_{p}^{\prime}, y_{1}, \ldots, y_{q}\right)}{Z\left(x_{1}, \ldots, x_{k}, y_{1}, \ldots, y_{q}\right)} d x_{k+1}^{\prime} \ldots d x_{p}^{\prime} \otimes \delta_{\left(y_{1}, \ldots, y_{q}\right)} .
\end{aligned}
$$

In the particular case where we do not want to condition the measure at all, the last expression is still true, with the convention that a conditional partition function without parameters is equal to 1 .

\subsubsection{Definition of the random holonomy}

Let $M_{1}$ be a minimal closure of $M$ endowed with a surface measure $\sigma_{1}$ that extends $\sigma$. We see $N_{1}, \ldots, N_{p}, L_{1}, \ldots, L_{q}$ as loops on $M_{1}$. There is a measurable space $\left(\Omega_{1}, \mathcal{A}_{1}\right)$ on which we constructed a family of measurable functions $\left(H_{c}\right)_{c \in P M_{1}}$. On this measurable space, we put the following probability:

$$
P_{1}=\int_{G^{p+q}} P\left(x_{1}^{\prime}, \ldots, x_{p}^{\prime}, y_{1}^{\prime}, \ldots, y_{q}^{\prime}\right) d\left(\beta\left(x_{1}, \ldots, x_{k}, y_{1}, \ldots, y_{q}\right)\right)\left(x_{1}^{\prime}, \ldots, x_{p}^{\prime}, y_{1}^{\prime}, \ldots, y_{q}^{\prime}\right) .
$$

In other words, we insist on the law of $\left(H_{N_{1}}, \ldots, H_{N_{p}}, H_{L_{1}}, \ldots, H_{L_{q}}\right)$ being the natural one under $P\left(x_{1}, \ldots, x_{k}, y_{1}, \ldots, y_{q}\right)$.

We consider the restriction of the family $\left(H_{c}\right)_{c \in C M_{1}}$ to $M$, i.e. we restrict the index set to $P M$.

Proposition 2.8.4 The law of the restriction $\left(H_{c}\right)_{c \in P M}$ does not depend on $M_{1}$. If $\Gamma=$ $\left\{a_{1}, \ldots, a_{r}\right\}$ is a graph on $M$ such that $L_{1}, \ldots, L_{q} \in \Gamma^{*}$, then the law of $\left(H_{a_{1}}, \ldots, H_{a_{r}}\right)$ under $P_{1}$ is the discrete Yang-Mills measure $P_{M}\left(x_{1}, \ldots, x_{k}, y_{1}, \ldots, y_{q}\right)$.

PROOF. The regularity property 2.6.5 of the random holonomy on $M_{1}$ is still true for its restriction to $M$. Thus, the second assertion implies the first one, using the fact that any family of paths on $M_{1}$ can be approximated by piecewise geodesic families.

Let $\Gamma$ be a graph as in the statement and $f$ be a continuous function on $G^{\Gamma}$.

$$
\begin{aligned}
E_{P_{1}} f\left(H_{a_{1}}, \ldots, H_{a_{r}}\right)= & \int_{G^{p+q}} \frac{d\left(\beta\left(x_{1}, \ldots, x_{k}, y_{1}, \ldots, y_{q}\right)\right)}{Z_{M_{1}}\left(x_{1}^{\prime}, \ldots, x_{p}^{\prime}, y_{1}^{\prime}, \ldots, y_{q}^{\prime}\right)} \int_{G^{\Gamma}} f\left(g_{1}, \ldots, g_{r}\right) \\
& \prod_{F \in \mathcal{F}(\Gamma), F \subset M_{1}} p_{\sigma_{1}(F)}\left(h_{\partial F}\right) d \nu_{x_{1}^{\prime}} \ldots d \nu_{x_{p}^{\prime}} d \nu_{y_{1}^{\prime}} \ldots d \nu_{y_{q}^{\prime}} d g^{\prime} .
\end{aligned}
$$


In $M_{1}$, the loops $N_{1}, \ldots, N_{p}$ bound $p$ disks $D_{1}, \ldots, D_{p}$ which are the only faces of $\Gamma$ that are not inside $M$. Thus,

$$
\begin{aligned}
Z_{M_{1}}\left(x_{1}^{\prime}, \ldots, x_{p}^{\prime}, y_{1}^{\prime}, \ldots, y_{q}^{\prime}\right) & =\int_{G^{\Gamma}} \prod_{F \in \mathcal{F}(\Gamma) F \subset M} p_{\sigma(F)}\left(h_{\partial F}\right) \prod_{i=1}^{p} p_{\sigma_{1}\left(D_{i}\right)}\left(h_{N_{i}}\right) \\
& =\prod_{i=1}^{p} p_{\sigma_{1}\left(D_{i}\right)}\left(x_{i}^{\prime}\right) Z_{M}\left(x_{1}^{\prime}, \ldots, \nu_{x_{p}^{\prime}}^{\prime}, y_{1}^{\prime}, \ldots, \nu_{y_{1}^{\prime}} \ldots d \nu_{y_{q}^{\prime}} d g^{\prime}\right.
\end{aligned}
$$

Using this last relation, we get:

$$
\begin{gathered}
E_{P_{1}} f\left(H_{a_{1}}, \ldots, H_{a_{r}}\right)=\int_{G^{p+q}} \frac{d\left(\beta\left(x_{1}, \ldots, x_{k}, y_{1}, \ldots, y_{q}\right)\right)}{Z_{M}\left(x_{1}^{\prime}, \ldots, x_{p}^{\prime}, y_{1}^{\prime}, \ldots, y_{q}^{\prime}\right)} \int_{G^{\Gamma}} f\left(g_{1}, \ldots, g_{r}\right) D_{M}^{\Gamma} \\
=\int_{G^{p-k}} \frac{Z\left(x_{1}, \ldots, x_{k}, x_{k+1}^{\prime}, \ldots, x_{p}^{\prime}, y_{1}, \ldots, y_{q}\right)}{Z\left(x_{1}, \ldots, x_{k}, y_{1}, \ldots, y_{q}\right)} \frac{d \nu_{y_{1}^{\prime}} \ldots d \nu_{y_{q}^{\prime}} d g^{\prime}}{Z\left(x_{1}, \ldots, x_{k}, x_{k+1}^{\prime}, \ldots, x_{p}^{\prime}, y_{1}, \ldots, y_{q}\right)} \\
\int_{G^{\Gamma}} f\left(g_{1}, \ldots, g_{r}\right) D_{M}^{\Gamma} d \nu_{x_{1}} \ldots d \nu_{x_{k}} d \nu_{x_{k+1}^{\prime}} \ldots d \nu_{x_{p}^{\prime}} d \nu_{y_{1}} \ldots d \nu_{y_{q}} d g^{\prime} \\
=\frac{1}{Z\left(x_{1}, \ldots, x_{k}, y_{1}, \ldots, y_{q}\right)} \int_{G^{\Gamma}} f\left(g_{1}, \ldots, g_{r}\right) D_{M}^{\Gamma} d \nu_{x_{1}} \ldots d \nu_{x_{k}} d \nu_{y_{1}} \ldots d \nu_{y_{q}} d g^{\prime} \\
=P\left(x_{1}, \ldots, x_{k}, y_{1}, \ldots, y_{q}\right)(f) .
\end{gathered}
$$

\subsection{Summary of the properties of the random holonomy}

\subsubsection{Existence, unicity in law and main properties}

Let us summarize what has been done in this chapter. We started with a surface $(M, \sigma)$, with or without boundary. We choosed on $M$ disjoint simple loops $L_{1}, \ldots, L_{q}$, whose image is either a boundary component of $M$ or contained in the interior of $M$. We picked $q$ elements $x_{1}, \ldots, x_{q}$ in $G$. We almost proved the following theorem:

Theorem 2.9.1 There exists a probabilty space $\left(\Omega, \mathcal{A}, P\left(x_{1}, \ldots, x_{q}\right)\right)$ and a family of $G$-valued random variables $\left(H_{c}\right)_{c \in P M}$ on this space, such that:

1. For any graph $\Gamma=\left\{a_{1}, \ldots, a_{r}\right\}$ on $M$ such that $L_{1}, \ldots, L_{q} \in \Gamma^{*}$, the law of $\left(H_{a_{1}}, \ldots, H_{a_{r}}\right)$ is the discrete Yang-Mills measure $P^{\Gamma}\left(x_{1}, \ldots, x_{q}\right)$ on $G^{\Gamma}$.

2. For any path $c$ of $P M$ and any sequence $\left(c_{n}\right)_{n \geq 0}$ of paths of $P M$ such that $c_{n} \underset{n \rightarrow \infty}{\stackrel{d_{1}}{\longrightarrow}} c$ with fixed end points, we have $H_{c_{n}} \underset{n \rightarrow \infty}{\stackrel{d_{P}}{\rightarrow}} H_{c}$.

The law of this family of random variables is uniquely defined by these two properties. Moreover, it has the following properties:

3. If $c_{1}$ and $c_{2}$ are paths that can be concatenated to form $c_{1} c_{2}$, then $H_{c_{1} c_{2}}=H_{c_{2}} H_{c_{1}}$ a.s.

4. If $\varphi: M \longrightarrow M$ is a diffeomorphism such that $\varphi_{*} \sigma=\sigma$, then $\varphi$ induces a permutation of the set of paths $P M$ and the families $\left(H_{c}\right)_{c \in P M}$ and $\left(H_{\varphi(c)}\right)_{c \in P M}$ have the same law. 
Proof. We already proved the existence of the family. When $M$ has a boundary, the probability space is that associated with a minimal closure of $M$. Let us prove the uniqueness in law. This law is a probability measure on the set $\mathcal{F}(P M, G)$ endowed with the $\sigma$-algebra generated by cylinder sets. So it is characterized by its finite-dimensional marginals. Since any family of paths can be approximated by families of paths in graphs, for example piecewise geodesic paths for some metric, the law of the random holonomy along an arbitrary finite family of paths is determined by properties (1) and (2).

Property (3) was already proved in proposition 2.6.4 for closed surfaces. For surfaces with boundary, the construction by restriction of the random holonomy on a minimal closure obviously preserves the multiplicativity.

Property (4) was proved at the discrete level in proposition 1.7.1. Since the law of the whole family is determined by discrete laws, it is also true is the continuous setting.

Given $(M, \sigma), L_{1}, \ldots, L_{q}, x_{1}, \ldots, x_{q}$, the law whose existence and uniqueness is stated by this theorem is a measure on $(\mathcal{F}(L M, G), \mathcal{C})$, where $\mathcal{C}$ is the $\sigma$-algebra generated by the cylinder sets. We shall denote this measure by $\mu_{0}\left(x_{1}, \ldots, x_{q}\right)$, or just $\mu_{0}$ if $q=0$. We keep the notation $\left(H_{c}\right)_{c \in P M}$ for the canonical process on the space $(\mathcal{F}(L M, G), \mathcal{C})$.

\subsubsection{Disintegration formula}

Consider a surface $(M, \sigma)$. Recall from proposition 1.5.3 that the conditional discrete measures constitute a disintegration of the free discrete Yang-Mills measure. We want to extend this result to the continuous setting. As usual, $L_{1}, \ldots, L_{q}$ are loops on $M$.

Proposition 2.9.2 The map $\left(x_{1}, \ldots, x_{q}\right) \mapsto \mu_{0}\left(x_{1}, \ldots, x_{q}\right)$ provides a disintegration of the measure $\mu_{0}$ on $(\mathcal{F}(P M, G), \mathcal{C})$ with respect to the random variable $\left(H_{L_{1}}, \ldots, H_{L_{q}}\right)$.

Proof. By construction, $\left(H_{L_{1}}, \ldots, H_{L_{q}}\right)=\left(x_{1}, \ldots, x_{q}\right) \mu_{0}\left(x_{1}, \ldots, x_{q}\right)$-a.s. Let $\left(c_{1}, \ldots, c_{n}\right)$ be a family of paths of $P M$. We need to prove that, for any function $f$ continuous on $G^{q}$,

$$
E_{\mu_{0}} f\left(H_{c_{1}}, \ldots, H_{c_{q}}\right)=\int_{G^{q}} E_{\mu_{0}\left(x_{1}, \ldots, x_{q}\right)} f\left(H_{c_{1}}, \ldots, H_{c_{q}}\right) d \eta\left(x_{1}, \ldots, x_{q}\right),
$$

where $\eta$ is the law of $\left(H_{L_{1}}, \ldots, H_{L_{q}}\right)$ under $\mu_{0}$. We already know that this result is true if $c_{1}, \ldots, c_{n}$ are paths in a graph. If they are not, we can approximate them in the $d_{1}$-topology by paths in graphs so that both expectations appearing in the formula converge. Since $G$ is compact, $f$ is bounded and the dominated convergence theorem applies.

\subsection{Yang-Mills measure}

\subsubsection{Definition of the Yang-Mills measure}

In this paragraph, we will explain why and how the measure $\mu_{0}$ defined in the preceding section still has to be transformed in order to become something that might be called Yang-Mills measure.

According to the formal description, Yang-Mills measure should be a measure on the quotient space $\mathcal{A} / \mathcal{J}$ of connections modulo gauge transformations. But an element of this space does 
not determine a holonomy along each path on $M$ that could be intrinsically represented by an element of $G$. Indeed, the holonomy along an open path $c$, i.e. such that $c(0) \neq c(1)$, can be transformed into any other $G$-equivariant diffeomorphism of the fiber over $c(0)$ into the fiber over $c(1)$ by an appropriate gauge transformation. The fact that the law of the random holonomy along an edge is always uniform on $G$ could be thought of as a reflect of this geometric property. This is why we will restrict to the set $L M$ of loops on $M$ instead of $P M$. Thus we will consider the family $\left(H_{l}\right)_{l \in L M}$ whose law is a probability measure on $(\mathcal{F}(L M, G), \mathcal{C})$, where we keep the notation $\mathcal{C}$ for the $\sigma$-algebra generated by the cylinders.

But it is still not true that an element of $\mathcal{A} / \mathcal{J}$ determines an element of $G$ as holonomy along each loop. Gauge transformations act by conjugation on the holonomy along loops. More precisely, they conjugate in the same way the holonomies along loops based at the same point. Let us denote by Ad the diagonal action of $G$ on $G^{n}$ defined by:

$$
\operatorname{Ad}(g)\left(g_{1}, \ldots, g_{n}\right)=\left(\operatorname{Ad}(g) g_{1}, \ldots, \operatorname{Ad}(g) g_{n}\right) .
$$

Orbits of this action will be called joint conjugacy classes and the joint class of $\left(g_{1}, \ldots, g_{n}\right)$ will be denoted by $\left[g_{1}, \ldots, g_{n}\right]$. We can reformulate our observation by saying that an element of $\mathcal{A} / \mathcal{J}$ determines the the joint conjugacy class of the holonomy along all loops based at the same point. Sengupta proved the converse of this statement (prop. 2.1.2 in [Se1]):

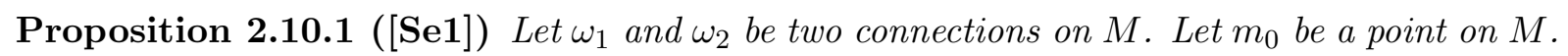
Suppose that along any finite family of loops $l_{1}, \ldots, l_{n}$ based at $m_{0}$, the joint conjugacy classes of the holonomies defined by $\omega_{1}$ and $\omega_{2}$ are equal. Then $\omega_{1}$ and $\omega_{2}$ belong to the same class in $\mathcal{A} / \mathcal{J}$.

Let the group $\mathcal{F}(M, G)$ act on $\mathcal{F}(L M, G)$ in the following way: if $j \in \mathcal{F}(M, G), f \in$ $\mathcal{F}(L M, G)$ and $l \in L M$, set

$$
(j \cdot f)(l)=j(l(0))^{-1} f(l) j(l(0)) .
$$

This action extends the action of a discrete gauge transformation. We can summarize our observations as follows:

Proposition 2.10.2 The holonomy allows to define an injective map

$$
\mathcal{A} / \mathcal{J} \longrightarrow \mathcal{F}(L M, G) / \mathcal{F}(M, G) .
$$

This result says that the quotient space $\mathcal{F}(L M, G) / \mathcal{F}(M, G)$ can be viewed as an extension of the space of connections modulo gauge transformations. We want to define the Yang-Mills measure on this space. To begin with, we must define a convenient $\sigma$-algebra.

There is a set of natural functions on the quotient space: given $n$ loops $l_{1}, \ldots, l_{n}$ based at the same point, the joint class $\left[H_{l_{1}}, \ldots, H_{l_{n}}\right]$ is a well-defined function that we denote by $\mathcal{H}_{l_{1}, \ldots, l_{n}}$. We will consider the $\sigma$-algebra $\mathcal{A}$ generated by the set of these functions. Of course, we want to be able to consider random variables associated with families of loops that are not based at the same point. We claim that the $\sigma$-algebra $\mathcal{A}$ allows to do this. Indeed, let $l_{1}, \ldots, l_{n}$ be a family of loops that we rewrite $\left(l_{1}, \ldots, l_{i_{1}}\right), \ldots,\left(l_{i_{p}+1}, \ldots, l_{n}\right)$, putting together the loops based at the same points. Then we can define the variable $\mathcal{H}_{l_{1}, \ldots, l_{n}}$ by

$$
\mathcal{H}_{l_{1}, \ldots, l_{n}}=\left(\mathcal{H}_{l_{1}, \ldots, l_{i_{1}}}, \ldots, \mathcal{H}_{l_{i_{p}+1}, \ldots, l_{n}}\right)
$$


and this random variable is measurable with respect to $\mathcal{A}$. Remark that $\mathcal{A}$ may also be seen as a $\sigma$-algebra on $\mathcal{F}(L M, G)$, invariant by the action of $\mathcal{F}(M, G)$, since the functions $\mathcal{H}_{l_{1}, \ldots, l_{q}}$ are also naturally defined on this space. Another natural choice for $\mathcal{A}$ would have been to consider the $\mathcal{F}(M, G)$-invariant sets of the cylinder $\sigma$-algebra $\mathcal{C}$. We shall discuss this point at the end of this section.

Proposition 2.10.3 Let $(M, \sigma)$ be a surface. Let $L_{1}, \ldots, L_{q}$ be disjoint simple loops on $M$ whose image is either a component of the boundary of $M$ or contained in the interior of $M$. Let $\left(x_{1}, \ldots, x_{q}\right)$ be an element of $G^{q}$. The restriction of $\mu_{0}\left(x_{1}, \ldots, x_{q}\right)$ to $\mathcal{A}$ depends on each $x_{i}$ only through its conjugacy class.

Proof. The point is to understand how $\mu_{0}\left(x_{1}, \ldots, x_{q}\right)$ is transformed under the action of $\mathcal{F}(M, G)$. Similarly to what we proved in 1.5.4, if $j$ is an element of $\mathcal{F}(M, G)$, then, setting $y_{i}=j\left(L_{i}(0)\right)$, we have

$$
j_{*} P\left(x_{1}, \ldots, x_{q}\right)=P\left(y_{1}^{-1} x_{1} y_{1}, \ldots, y_{q}^{-1} x_{q} y_{q}\right) .
$$

Indeed, we already know that this equality holds when we evaluate these measures against functions of the holonomy along paths in a graph, and we extend it to general measurable functions by the usual approximation scheme.

Thus, the $\mu_{0}\left(x_{1}, \ldots, x_{q}\right)$-measure of sets invariant under the action of $\mathcal{F}(M, G)$ depends only on the conjugacy classes $\left[x_{1}\right], \ldots,\left[x_{q}\right]$.

We denote by $t_{i}$ the conjugacy class of each $x_{i}$.

Definition 2.10.4 We call Yang-Mills measure on $M$ and denote by $\mu$ the image measure of $\mu_{0}$ on the quotient space $(\mathcal{F}(L M, G) / \mathcal{F}(M, G), \mathcal{A})$, or equivalently the restriction of $\mu_{0}$ to $(\mathcal{F}(L M, G), \mathcal{A})$.

Similarly, we call conditional Yang-Mills measure with respect to $L_{1}, \ldots, L_{q}$ and we denote by $\mu\left(t_{1}, \ldots, t_{q}\right)$ the image measure of $\mu_{0}\left(x_{1}, \ldots, x_{q}\right)$ on the quotient space $(\mathcal{F}(L M, G) / \mathcal{F}(M, G), \mathcal{A})$, or equivalently the restriction of $\mu_{0}\left(x_{1}, \ldots, x_{q}\right)$ to $(\mathcal{F}(L M, G), \mathcal{A})$.

The first point of view keeps track of the quotient structure of the space $\mathcal{A} / \mathcal{J}$. Nevertheless, the second will often be technically more convenient.

Proposition 2.10.5 The map $\left(t_{1}, \ldots, t_{q}\right) \mapsto \mu\left(t_{1}, \ldots, t_{q}\right)$ defined on $(G / \mathrm{Ad})^{q}$ provides a disintegration of the measure $\mu$ with respect to the random variable $\mathcal{H}_{L_{1}}, \ldots, \mathcal{H}_{L_{q}}$.

Note that since the $L_{i}$ 's are not based at the same point, the variables $\mathcal{H}_{L_{1}, \ldots, L_{q}}$ and $\left(\mathcal{H}_{L_{1}}, \ldots, \mathcal{H}_{L_{q}}\right)$ are equal.

Proof. Let $\left(t_{1}, \ldots, t_{q}\right)$ be an element of $(G / \mathrm{Ad})^{q}$ and $\left(x_{1}, \ldots, x_{q}\right) \in G^{n}$ be such that $\left[x_{i}\right]=t_{i}$ for each $i$. Then $\left(\left[H_{L_{1}}\right], \ldots,\left[H_{L_{q}}\right]\right)=\left(\left[x_{1}\right], \ldots,\left[x_{q}\right]\right) \mu_{0}\left(x_{1}, \ldots, x_{q}\right)$-a.s., so that

$$
\left(\mathcal{H}_{L_{1}}, \ldots, \mathcal{H}_{L_{q}}\right)=\left(t_{1}, \ldots, t_{q}\right) \mu\left(t_{1}, \ldots, t_{q}\right)-\text { a.s. }
$$

By 1.5.2, we know that the law of $\left(H_{L_{1}}, \ldots, H_{L_{q}}\right)$ under $\mu_{0}$ is $Z^{-1} Z\left(x_{1}, \ldots, x_{q}\right) d x_{1} \ldots d x_{q}$. We also proved in 2.9 .2 that $\mu_{0}$ is disintegrated by the $\mu_{0}\left(x_{1}, \ldots, x_{q}\right)$, so that

$$
\mu_{0}=\frac{1}{Z} \int_{G^{q}} Z\left(x_{1}, \ldots, x_{q}\right) \mu_{0}\left(x_{1}, \ldots, x_{q}\right) d x_{1} \ldots d x_{q} .
$$


If we evaluate these measures on sets of $\mathcal{A}$ and use the invariance by conjugation of the conditional partition function stated in 1.5.5, we find:

$$
\begin{aligned}
\mu_{0} & =\frac{1}{Z} \int_{G^{q}} Z\left(\left[x_{1}\right], \ldots,\left[x_{q}\right]\right) \mu_{0}\left(\left[x_{1}\right], \ldots,\left[x_{q}\right]\right) d x_{1} \ldots d x_{q} \\
& =\frac{1}{Z} \int_{(G / \mathrm{Ad})^{q}} Z\left(t_{1}, \ldots, t_{q}\right) \mu_{0}\left(t_{1}, \ldots, t_{q}\right) d t_{1} \ldots d t_{q}
\end{aligned}
$$

where $d t$ is the image measure on $G$ / Ad of the Haar measure. The last equality restricted to $\mathcal{A}$-measurable sets proves the result.

\subsubsection{Regularity properties}

In order to study Yang-Mills measure, we need to say more about the set of joint conjugacy classes $G^{n} / \mathrm{Ad}$. We regard it as a set of compact subsets of $G^{n}$ and endow it with the Hausdorff distance, defined in general between two compact sets by

$$
d\left(K_{1}, K_{2}\right)=\sup \left(\sup _{k_{1} \in K_{1}} \inf _{k_{2} \in K_{2}} d\left(k_{1}, k_{2}\right), \sup _{k_{2} \in K_{2}} \inf _{k_{1} \in K_{1}} d\left(k_{1}, k_{2}\right)\right)
$$

Lemma 2.10.6 The canonical projection $G^{n} \longrightarrow G^{n} / \operatorname{Ad}$ is 1-Lipchitz.

Proof. Let $\left(g_{1}, \ldots, g_{n}\right)$ and $\left(h_{1}, \ldots, h_{n}\right)$ be two elements of $G^{n}$.

$$
\begin{aligned}
d\left(\left[g_{1}, \ldots, g_{n}\right],\left[h_{1}, \ldots, h_{n}\right]\right) & =\sup _{g \in G} \inf _{h \in G} d\left(\operatorname{Ad}(g)\left(g_{1}, \ldots g_{n}\right), \operatorname{Ad}(h)\left(h_{1}, \ldots, h_{n}\right)\right) \\
& =\inf _{h \in G} d\left(\left(g_{1}, \ldots, g_{n}\right), \operatorname{Ad}(h)\left(h_{1}, \ldots, h_{n}\right)\right) \\
& \leq d\left(\left(g_{1}, \ldots, g_{n}\right),\left(h_{1}, \ldots, h_{n}\right)\right) .
\end{aligned}
$$

As before, this distance on $G^{n} /$ Ad allows to define the distance

$$
d_{P}(X, Y)=E d(X, Y)
$$

between $G^{n} /$ Ad-valued random variables defined on the same probability space. The regularity property 2.6.5 of the random holonomy becomes the following regularity property for the YangMills measure:

Proposition 2.10.7 Let $\left(\left(l_{1, k}, \ldots, l_{n, k}\right)\right)_{k \geq 0}$ be a sequence of $n$-uples of loops such that

1. for each $k \geq 0$, the loops $l_{1, k}, \ldots, l_{n, k}$ are based at the same point, Then

2. for each $i=1, \ldots, n$, there exists a loop $l_{i}$ such that $l_{i, k} \underset{k \rightarrow \infty}{\stackrel{d_{1}}{\longrightarrow}} l_{i}$.

$$
\mathcal{H}_{l_{1, k}, \ldots, l_{n, k}} \underset{k \rightarrow \infty}{\stackrel{d_{P}}{\rightarrow}} \mathcal{H}_{l_{1}, \ldots, l_{n}}
$$


Proof. The loops $l_{i}$ are necessarily based at the same point, denoted by $m$. Denoting by $m_{k}$ the base point of the $l_{i, k}$ 's, we have $m_{k} \longrightarrow m$. For each $k$, let $z_{k}$ denote an arbitrary path joining $m$ to $m_{k}$. Then for each $i z_{k} l_{i, k} z_{k}^{-1} \stackrel{d_{1}}{\longrightarrow} l_{i}$ with fixed basepoint, so that

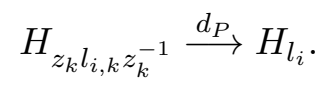

Since the projection on $G^{n} /$ Ad reduces the distances, this implies

$$
\left[H_{z_{k} l_{1, k} z_{k}^{-1}}, \ldots, H_{z_{k} l_{n, k} z_{k}^{-1}} \underset{k \rightarrow \infty}{\stackrel{d_{P}}{\longrightarrow}}\left[H_{l_{1}}, \ldots, H_{l_{n}}\right] .\right.
$$

The left hand side term is equal to

$$
\left[H_{z_{k}}^{-1} H_{l_{1, k}} H_{z_{k}}, \ldots, H_{z_{k}}^{-1} H_{l_{n, k}} H_{z_{k}}\right]=\left[H_{l_{1, k}}, \ldots, H_{l_{n, k}}\right]
$$

so that the result is proved.

\subsubsection{Remarkable subfamilies of random variables}

We study two special subfamilies of random variables defined on $(\mathcal{F}(L M, G) / \mathcal{F}(M, G), \mathcal{A}, \mu)$, using the results proved in the preceding paragraphs.

We begin by the family $\left(\mathcal{H}_{l}\right)_{l \in L M}$. Each variable is $G / \mathrm{Ad}$-valued and this family satisfies a very nice regularity property:

Proposition 2.10.8 Let $l$ be in $L M$ and $\left(l_{n}\right)_{n \geq 0}$ be a sequence of loops that converges to $l$. Then $\mathcal{H}_{l_{n}} \stackrel{d_{P}}{\longrightarrow} \mathcal{H}_{l}$.

This is the only situation where we can forget about end points. Unfortunately, this family does not generate $\mathcal{A}$, since it does not contain any information about joint conjugacy classes.

Now fix a point $m \in M$ and consider the set $L_{m} M$ of loops based at $m$. We are interested in the family $\left(\mathcal{H}_{l_{1}, \ldots, l_{n}}\right)_{l_{i} \in L_{m} M}$. It has the same property as that stated in 2.10.7, the condition on end points being always satisfied. What is interesting here is the following fact:

Proposition 2.10.9 The family $\left(\mathcal{H}_{l_{1}, \ldots, l_{n}}\right)_{l_{i} \in L_{m} M}$ generates the $\sigma$-algebra $\mathcal{A}$.

Proof. Let $l_{1}, \ldots, l_{n}$ be $n$ loops on $M$ based at a point $m_{1}$. Let $c$ be a path joining $m$ to $m_{1}$. Then the equality

$$
\mathcal{H}_{c l_{1} c^{-1}, \ldots, c l_{n} c^{-1}}=\mathcal{H}_{l_{1}, \ldots, l_{n}}
$$

proves that it is always possible to get back to loops based at $m$.

This subfamily satisfies also a multiplicativity property. Indeed, the joint conjugacy class of some elements of $G$ determines the joint class of all products of these elements. For example, there is a well defined map from $G^{n} / \mathrm{Ad}$ to $G^{n-1} / \mathrm{Ad}$ that sends $\left[g_{1}, \ldots, g_{n-1}, g_{n}\right]$ to $\left[g_{1}, \ldots, g_{n-2}, g_{n} g_{n-1}\right]$. The multiplicativity can be expressed by saying that for any $l_{1}, \ldots, l_{n} \in$ $L_{m} M$, this maps sends almost surely $\mathcal{H}_{l_{1}, \ldots, l_{n}}$ to $\mathcal{H}_{l_{1}, \ldots, l_{n-1} l_{n}}$. 


\section{Remark.}

Let us discuss the definition of the $\sigma$-algebra $\mathcal{A}$. For this, we consider the Yang-Mills measure an invariant measure on the space $\mathcal{F}\left(L_{m} M, G\right)$, because in this setting, the action of the gauge group is that of the finite dimensional group $G$. We use this fact below in order to integrate functions over the orbits of this action.

We could have made another natural choice of an invariant $\sigma$-algebra on the space $\mathcal{F}\left(L_{m} M, G\right)$, namely that of invariant sets of the cylinder $\sigma$-algebra $\mathcal{C}$ on $\mathcal{F}\left(L_{m} M, G\right)$. Let us denote by $\mathcal{C}_{\mathcal{I}}$ this $\sigma$-algebra. It is clear that $\mathcal{A} \subset \mathcal{C}_{\mathcal{I}}$ and it is very likely that this inclusion is in fact an equality. We prove that the completions $\widetilde{\mathcal{A}}$ and $\widetilde{\mathcal{C}_{\mathcal{I}}}$ with respect to $\mu_{M}(x)$ are equal.

We use the separability of $L_{m} M$ proved in the next lemma.

Lemma 2.10.10 Let $M$ be a surface and $m$ a point of $M$. The loop space $L_{m} M$ endowed with the $d_{1}$-topology is separable.

Proof. We construct a countable dense subset of $L_{m} M$. The first point is that $M$ itself is separable. Choose a countable dense subset $\Pi \subset M$ containing $m$. Endow $M$ with a Riemannian metric such that $\partial M$ is geodesic if it is non empty. Let $R_{M}$ be the convexity radius of $M$ : two points at distance smaller than $R_{M}$ are joined by a unique minimizing geodesic. Define $\Lambda$ to be the set of loops obtained by concatenation of a finite number of geodesic segments joining two points of $\Pi$ at distance smaller than $R_{M}$. The set $\Lambda$ is countable because it is equipotent to a subset of finite sequences of $\Pi$. We claim that it is dense in $L_{m} M$. Indeed, any geodesic segment of length smaller than $R_{M}$ can be approximated by segments joining points of $\Pi$, since a small piece of geodesic depends continuously on its end points. Thus, the $d_{1}$-closure of $\Lambda$ contains the set of piecewise geodesic loops and we already know that this set is dense in $L_{m} M . \square$

Let $\Lambda \subset L_{m} M$ be a countable dense subset. Let $\mathcal{A}^{\Lambda}$ denote $\sigma\left(\mathcal{H}_{\lambda_{1}, \ldots, \lambda_{n}} \in \Lambda\right)$. Let $\mathcal{C}^{\Lambda}$ denote $\sigma\left(H_{\lambda}, \lambda \in \Lambda\right)$ and $\left(\mathcal{C}^{\Lambda}\right)_{\mathcal{I}}$ denote the invariant sets of this $\sigma$-algebra. It is clear that $\mathcal{A}^{\Lambda} \subset\left(\mathcal{C}^{\Lambda}\right)_{\mathcal{I}}$. It is also clear that $\mathcal{A}^{\Lambda}$ is a separable $\sigma$-algebra. Finally, one easily checks that any atom of $\mathcal{A}^{\Lambda}$ is contained in an atom of $\left(\mathcal{C}^{\Lambda}\right)_{\mathcal{I}}$. Thus, Blackwell's theorem implies (see [DM]) that $\mathcal{A}^{\Lambda}=\left(\mathcal{C}^{\Lambda}\right)_{\mathcal{I}}$.

We use this equality to prove the inclusion $\mathcal{C}_{\mathcal{I}} \subset \widetilde{\mathcal{A}}$, which implies the result. The point is that the continuity in probability of the map $l \mapsto \mathcal{H}_{l}$ and the density of $\Lambda$ in $L_{m} M$ imply that $\widetilde{\mathcal{C}^{\Lambda}}$ contains $\mathcal{C}$ and that $\widetilde{\mathcal{A}^{\Lambda}}$ contains $\mathcal{A}$. This last inclusion implies the equality $\overline{\mathcal{A}^{\Lambda}}=\widetilde{\mathcal{A}}$. Thus, it is sufficient to prove that $\mathcal{C}_{\mathcal{I}} \subset \widetilde{\mathcal{A}^{\Lambda}}$. Let $f$ be a $\mathcal{C}_{\mathcal{I}}$-measurable function. As a $\mathcal{C}$-measurable function, it is $\mu_{M}(x)$-almost surely the limit of a sequence $\left(f_{n}\right)$ of $\mathcal{C}^{\Lambda}$-measurable functions. Let us integrate these functions $f_{n}$ over the orbits of the action of $G$ on $\mathcal{F}\left(L_{m} M, G\right)$, using the Haar measure on $G$. We get a sequence of $\left(\mathcal{C}^{\Lambda}\right)_{\mathcal{I}^{-}}$-measurable functions still converging to $f$. Thus, $f$ is measurable with respect to the completion $\left(\widetilde{\mathcal{C}^{\Lambda}}\right)_{\mathcal{I}}=\widetilde{\mathcal{A}^{\Lambda}}$ and we get the result. 


\section{Chapter 3}

\section{Abelian theory}

In this chapter, we continue the investigation of the case $G=U(1)$ started in section 1.9. Recall that we had reconstructed the random holonomy along loops homologous to zero in a graph, using a white noise on $M$ (see proposition 1.9.10). We extend now this reconstruction to all cycles of $M$, using the unicity properties of the Yang-Mills measure proved in chapter 2.

Then we show that it is possible to proceed backwards, namely to extract a white noise on $M$ from the Yang-Mills measure on $M$, more precisely, using the random holonomy along very small loops. This makes clear the relationship between the random holonomy and the white noise in this Abelian case.

\subsection{The random holonomy as a white noise functional}

As usual, $M$ may have a boundary $\partial M=N_{1} \cup \ldots \cup N_{p}$. Choose elements $x_{1}, \ldots, x_{p}$ in $U(1)$ and set $x=x_{1} \ldots x_{p}$ or $x=1$ if $M$ has no boundary. We denote by $C M$ the set of cycles on $M$, i.e. the set of linear combination of loops with integer coefficients and by $C_{0} \Gamma$ the set of cycles homologous to zero. The family of random variables $\left(H_{l}\right)_{l \in L M}$, that we used in 2.10 to construct the Yang-Mills measure $\mu_{M}\left(x_{1}, \ldots, x_{p}\right)$, extends by multiplicativity to the cycles of $C M$ and gives rise to a measure on $\mathcal{F}(C M, U(1))$. In this Abelian setting, the action of $\mathcal{F}(M, U(1))$ is trivial.

We seek a result similar to 1.9 .9 , valid for all cycles on $M$. We begin by defining a family of random variables using a white noise on $M$ and prove later that it has the law of a Yang-Mills random holonomy.

Recall that we proved earlier that the holonomies along a system of loops representing a basis of $H_{1}(M ; \mathbf{Z})$ are independent uniform variables on $U(1)$, independent of the holonomies along loops homologous to zero (see proposition 1.9.4).

\subsubsection{Definition of the white noise functional}

Recall that $g$ denotes the genus of $M$. In order to define the double layer potential (see 1.9.6), we need a Riemannian metric on $M$, that we choose such that the boundary of $M$ is geodesic. Let $\ell_{1}, \ldots, \ell_{2 g}$ be piecewise geodesic loops such that

$$
\mathcal{B}=\left(\left[\ell_{1}\right], \ldots,\left[\ell_{2 g}\right],\left[N_{1}\right], \ldots,\left[N_{p-1}\right]\right)
$$

is a basis of $H_{1}(M ; \mathbf{Z})$. 
Let $c$ be a cycle of $C M$. We can decompose it in $H_{1}(M ; \mathbf{Z})$ :

$$
[c]=\lambda_{1}\left[\ell_{1}\right]+\ldots+\lambda_{2 g}\left[\ell_{2 g}\right]+\nu_{1}\left[N_{1}\right]+\ldots+\nu_{p-1}\left[N_{p-1}\right] .
$$

Let $U_{1}, \ldots, U_{2 g}$ be $2 g$ independent uniform random variables on $U(1)$. Set

$$
\Theta_{c}=U_{1}^{\lambda_{1}} \ldots U_{2 g}^{\lambda_{2 g}} x_{1}^{\nu_{1}} \ldots x_{p-1}^{\nu_{p-1}}
$$

There is a cycle $c^{\perp}$ of $C_{0} M$, i.e. a cycle homologous to zero, associated with $c$, defined by

$$
c^{\perp}=c-\left(\lambda_{1} \ell_{1}+\ldots+\lambda_{2 g} \ell_{2 g}+\nu_{1} N_{1}+\ldots+\nu_{p-1} N_{p-1}\right) .
$$

Recall that $u_{c^{\perp}}$ denotes the doule layer potential of $c^{\perp}$. The cycle $c^{\perp}$ is the boundary of a 2-chain $\alpha$. We defined an element $\sigma_{\text {int }}\left(c^{\perp}\right)$ of $\mathbf{R}$ (resp. $\mathbf{R} / \mathbf{Z}$ ) when $M$ has a boundary (resp. no boundary) by $\sigma_{\text {int }}\left(c^{\perp}\right)=\frac{|\langle\sigma, \alpha\rangle|}{\sigma(M)}$.

Let $W$ be a white noise on $M$, independent of the $U_{i}$ 's. Let $T$ be a variable independent of $W$ and the $U_{i}$ 's, whose law is that described in proposition 1.9.5. Finally, denote by $W_{0}$ the projection of $W$ on the hyperplane of zero-mean functions: for any function $u \in L^{2}(M, \sigma)$,

$$
W_{0}(u)=W\left(u-\frac{1}{\sigma(M)} \int_{M} u d \sigma\right) .
$$

We are able to define what will be proved to be a second realization of the random holonomy along cycles. Denote by $(\Omega, P)$ a probability space that supports $W, T$ and the $U_{i}$ 's.

Definition 3.1.1 For each cycle $c \in C M$, define the following random variable on $(\Omega, P)$ :

$$
W H_{c}=\exp i\left(W_{0}\left(u_{c^{\perp}}\right)+\sigma_{\mathrm{int}}\left(c^{\perp}\right) T\right) \Theta_{c} .
$$

\subsubsection{Regularity of the new random holonomy}

In order to prove that this family has the law of the random holonomy, we will check that this is true for a restricted class of paths, namely piecewise geodesic paths, and extend this partial result by continuity. This is why we are interested in the regularity of this new family.

We begin by extending the distance $d_{1}$ to the space $C M$ of cycles.

Definition 3.1.2 Let $c=n_{1} l_{1}+\ldots+n_{k} l_{k}$ and $c^{\prime}=n_{1}^{\prime} l_{1}^{\prime}+\ldots+n_{k^{\prime}}^{\prime} l_{k^{\prime}}^{\prime}$ be two cycles on $M$, written as combinations of loops. If $k \neq k^{\prime}$, set $d_{1}\left(c, c^{\prime}\right)=1$. If $k=k^{\prime}$, let $\pi\left(c, c^{\prime}\right)$ be the set of permutations $\tau \in S_{k}$ such that $n_{\tau(i)}^{\prime}=n_{i}$ for all $i=1, \ldots, k$. If $\pi\left(c, c^{\prime}\right)=\emptyset$, set $d_{1}\left(c, c^{\prime}\right)=1$. Otherwise, set

$$
d_{1}\left(c, c^{\prime}\right)=\inf _{\tau \in \pi\left(c, c^{\prime}\right)} \sum_{i=1}^{k} n_{i} d_{1}\left(l_{i}, l_{\tau(i)}^{\prime}\right) .
$$

We will use proposition 2.6.8 about the continuity of the double layer potential of loops to prove the next proposition. Recall that 2.6 .8 was proved only on surfaces without boundary.

Proposition 3.1.3 Suppose that $M$ has no boundary. Let c be a cycle and $\left(c_{n}\right)_{n \geq 0}$ be a sequence of cycles such that $c_{n} \stackrel{d_{1}}{\longrightarrow}$ c. Then $u_{c_{n}} \stackrel{L^{2}}{\longrightarrow} u_{c}$. 
Proof: Decompose $c$ as $n_{1} l_{1}+\ldots+n_{k} l_{k}$. Let $N=n_{1}+\ldots+n_{k}$. Fix $\varepsilon>0$. For each $l_{i}$, there exists $\delta_{i}<1$ such that $d_{1}\left(l_{i}^{\prime}, l_{i}\right)<\delta_{i}$ implies $\left\|u_{l_{i}^{\prime}}-u_{l_{i}}\right\|_{L^{2}}<\frac{\varepsilon}{k N}$ for any loop $l_{i}^{\prime}$. Suppose that $n$ is such that $d_{1}\left(c_{n}, c\right)<\inf \delta_{i}$. Then $c_{n}$ can be written $n_{1} l_{1}^{\prime}+\ldots+n_{k} l_{k}^{\prime}$, with $\sum n_{i} d_{1}\left(l_{i}, l_{i}^{\prime}\right)<\inf \delta_{i}$. For each $i$, we have in particular $d_{1}\left(l_{i}, l_{i}^{\prime}\right)<\delta_{i}$. Thus,

$$
\left\|u_{c_{n}}-u_{c}\right\|_{L^{2}} \leq \sum_{i=1}^{k} n_{i}\left\|u_{l_{i}^{\prime}}-u_{l_{i}}\right\|_{L^{2}}<\frac{k N \varepsilon}{k N}=\varepsilon .
$$

The homology class of a cycle $c$ depends continuously on $c$, even for the distance $d_{\infty}$. Thus, $c^{\perp}$ and hence $u_{c^{\perp}}$ depends continuously on $c$, and, by continuity of the white noise which is an isometry, the map $c \mapsto W_{0}\left(u_{c^{\perp}}\right)$ is continuous for the $L^{2}$ norm when $M$ is closed. We want to extend this result to surfaces with boundary. For this, we study $u_{c}$ when $c$ is homologous to zero.

Let $M$ be a surface, with or without boundary and fix $c \in C_{0} M$. Let $x$ and $y$ be two points of $M$ outside the image of $c$. If necessary, we modify locally the $\ell_{i}$ 's in a neighbourhood of $x$ in order to make sure that $x$ meets none of them. Let $\ell_{x}$ be the boundary of a small disk $D_{x}$ around $x$, small enough not to meet $c$ and not to contain $y$. The module $H_{1}(M-\{x, y\})$ is generated by $\left[\ell_{1}\right], \ldots,\left[\ell_{2 g}\right],\left[N_{1}\right], \ldots,\left[N_{p-1}\right],\left[\ell_{x}\right],\left[\ell_{y}\right]$. In $M-\{x, y\}$, we have the equality

$$
[c]=\sum_{i=1}^{2 g} \lambda_{i}\left[\ell_{i}\right]+\sum_{j=1}^{p-1} \nu_{j}\left[N_{j}\right]+p\left[\ell_{x}\right]+q\left[\ell_{y}\right]
$$

for some $p \in \mathbf{Z}$. This equality also holds in $M$, where $[c]=\left[\ell_{x}\right]=\left[\ell_{y}\right]=0$, and this proves that $\lambda_{i}=\nu_{j}=0$. Thus, $[c]=p\left[\ell_{x}\right]+q\left[\ell_{y}\right]$ in $M-\{x, y\}$.

Lemma 3.1.4 With the preceding notations, $u_{c}(x)-u_{c}(y)=p-q$.

Proof: Recall the definition of the Green function $G(\cdot, \cdot)$ from equation (1.9), and the value of the double layer potential of small loops from proposition 1.9.7. The 1-form $* d G_{x}-* d G_{y}$ is closed on $M-\{x, y\}$. So,

$$
\begin{aligned}
u_{c}(x)-u_{c}(y) & =\int_{c} * d G_{x}-* d G_{y} \\
& =p \int_{\ell_{x}} * d G_{x}-* d G_{y}+q \int_{\ell_{y}} * d G_{x}-* d G_{y} \\
& =p\left(u_{\ell_{x}}(x)-u_{\ell_{x}}(y)\right)+q\left(u_{\ell_{y}}(x)-u_{\ell_{y}}(y)\right) \\
& =p\left(\mathbf{1}_{D_{x}}(x)-\frac{\sigma\left(D_{x}\right)}{\sigma(M)}-\mathbf{1}_{D_{x}}(y)+\frac{\sigma\left(D_{x}\right)}{\sigma(M)}\right)+q\left(\mathbf{1}_{D_{y}}(x)-\mathbf{1}_{D_{y}}(y)\right) \\
& =p-q .
\end{aligned}
$$

\section{Remark.}

If $M$ is closed, then $\left[\ell_{x}\right]+\left[\ell_{y}\right]=0$ in $H_{1}(M-\{x, y\})$. In this case, $p$ and $q$ are defined up to an additive constant but the difference $p-q$ is well defined. 
Corollary 3.1.5 1. When $M$ has no boundary, the double layer potential of cycles of $C_{0} M$ does not depend on the choice of the metric.

2. The potential $u_{c}$ is constant on each connected component of the complementary of the image of $c$.

3. If $M_{1}$ is a minimal closure of $M$ and if we identify $M$ with a submanifold of $M_{1}$, then for any $c \in C_{0} M$, the potentials $u_{c}^{M}$ and $u_{c}^{M_{1}}{ }_{\mid M}$ computed respectively in $M$ and $M_{1}$ differ only by an additive constant.

Proof. 1. The lemma determines the potential of any cycle of $C_{0} M$ up to a constant. When $M$ has no boundary this constant is determined by the condition $\int_{M} u_{c} d \sigma=0$.

2. Given a fixed point $y_{0}, u_{c}(x)-u_{c}\left(y_{0}\right)$ depends only on the homology class of $c$ in $M-\{x, y\}$, which does not change if $x$ stays in a given connected component.

3. Both functions $u_{c}^{M}$ and $u_{c}^{M_{1}}{ }_{\mid M}$ satisfy the property shown in the lemma, with the same values of $p$ and $q$. Indeed, if $[c]=p\left[\ell_{x}\right]+q\left[\ell_{y}\right]$ in $H_{1}(M)$, then the same equality holds in $H_{1}(M-\{x, y\})$. Thus, they cannot differ by more than an additive constant.

Proposition 3.1.6 Even if $M$ has a boundary, the map $c \mapsto W_{0}\left(u_{c^{\perp}}\right)$ is continuous.

PROOF: By property (3) of the preceding corollary,

$$
u_{c^{\perp}}^{M}-\frac{1}{\sigma(M)} \int_{M} u_{c^{\perp}}^{M} d \sigma=u_{c^{\perp} \mid M}^{M_{1}}-\frac{1}{\sigma(M)} \int_{M} u_{c^{\perp}}^{M_{1}} d \sigma .
$$

Together with 3.1.3, this shows that $u_{c^{\perp}}$ depends continuously on $c_{0}$. This was the only missing point.

Now we study the term $\sigma_{\text {int }}(c)$, when $c \in C_{0} M$. We will show that it can be extracted from the double layer potential of $c$.

Lemma 3.1.7 Let $c$ be a cycle of $C_{0} M$.

1. If $M$ has no boundary, $\sigma_{\text {int }}(c)$ is the element $t \in \mathbf{R} / \mathbf{Z}$ such that $u_{c}$ takes its values in $\mathbf{Z}-t$.

2. If $M$ has a boundary, consider $M_{1}$ a minimal closure of $M$. Then $\sigma_{\text {int }}(c)$ is equal to $-\frac{\sigma\left(M_{1}\right)}{\sigma(M)}$ times the value of $u_{c}^{M_{1}}$ at any point of $M_{1}-M$.

Proof: 1 . Let $x$ be a point of $M$ outside the image of $c$. Denote by $\alpha$ a 2 -chain such that $c=\partial \alpha$. Then

$$
\begin{aligned}
u_{c}(x)=\int_{c} * d G_{x} & =\int_{\alpha} \delta_{x}-\frac{d \sigma}{\sigma(M)} \\
& \equiv-\frac{|\langle\sigma, \alpha\rangle|}{\sigma(M)}(\bmod 1)
\end{aligned}
$$

2. Let $x$ be a point of $M_{1}-M$. We have

$$
u_{c}^{M_{1}}(x)=\int_{\alpha} \delta_{x}-\frac{d \sigma}{\sigma\left(M_{1}\right)}=-\frac{|\langle\sigma, \alpha\rangle|}{\sigma\left(M_{1}\right)}=-\frac{\sigma(M)}{\sigma\left(M_{1}\right)} \sigma_{\mathrm{int}}(c) .
$$


Let $\left(c_{n}\right)_{n \geq 0}$ be a sequence of cycles of $C_{0} M$ such that $c_{n} \longrightarrow c$. Since $u_{c_{n}} \stackrel{L^{2}}{\longrightarrow} u_{c}$ and since all these functions are locally constant, there is pointwise convergence outside the image of $c$. This implies:

Proposition 3.1.8 The map $c \mapsto \sigma_{\text {int }}(c)$ defined on $C_{0} M$ is continuous.

Finally, the map $c \mapsto \Theta_{c}$ is locally constant on $C_{0} M$. We proved:

Proposition 3.1.9 The map $c \mapsto W H_{c}$ is continuous from $\left(C M, d_{1}\right)$ into the space of square integrable random variables on $(\Omega, P)$.

The last property that we need is the multiplicativity:

Proposition 3.1.10 For any cycles $c_{1}$ and $c_{2}$ in $C M$,

$$
W H_{c_{1}+c_{2}}=W H_{c_{1}} W H_{c_{2}} P-\text { a.s. }
$$

Proof: This follows immediately from the following facts: $c^{\perp}$ depends linearly on $c$, the double layer potential is additive, the map $c \mapsto \sigma(c)$ is also additive and the map $c \mapsto \Theta_{c}$ is multiplicative.

\subsubsection{Identification of the random holonomies}

We are now able to prove the main theorem of this section:

Theorem 3.1.11 The family of random variables $\left(W H_{c}\right)_{c \in C M}$ has the same law as the family $\left(\mathcal{H}_{c}\right)_{c \in C M}$ under $\mu_{M}\left(x_{1}, \ldots, x_{p}\right)$.

PROOF: According to the unicity statement of the theorem 2.9.1 and to the regularity property 3.1.9, it is enough to prove the equality of the laws for piecewise geodesic cycles. Let $\Gamma$ be a piecewise geodesic graph such that $\ell_{1}, \ldots, \ell_{2 g} \in \Gamma^{*}$. Recall that these loops generate the first homology group of a minimal closure of $M$. Denote by $F_{1}, \ldots, F_{n}$ the faces of $\Gamma$. The arguments developped in 1.9.1 explain why it is enough to prove the equality of the laws for the fundamental system $\left(\ell_{1}, \ldots, \ell_{2 g}, N_{1}, \ldots, N_{p}, \partial F_{1}, \ldots, \partial F_{n}\right)$.

On one hand, $\ell_{1}^{\perp}=\ldots=\ell_{2}^{\frac{\perp}{2 g}}=0$, so that $W H_{\ell_{i}}=U_{i}$ for all $i=1, \ldots, 2 g$. On the other hand, $N_{1}^{\perp}=\ldots=N_{p-1}^{\perp}=0$ and $N_{p}^{\perp}=N_{1}+\ldots+N_{p}$. Thus, $\sigma_{\text {int }}\left(N_{p}^{\perp}\right)=1$ and $u_{N_{p}^{\perp}}$ is equal to zero so that $W_{0}\left(u_{N_{p}^{\perp}}\right)=0$. This implies $W H_{N_{j}}=x_{j}$ for all $j=1, \ldots, p-1$ and also for $j=p$. Finally, we already proved in 1.9 .9 that $\left(W H_{\partial F_{1}}, \ldots, W H_{\partial F_{n}}\right)$ and $\left(\mathcal{H}_{\partial F_{1}}, \ldots, \mathcal{H}_{\partial F_{n}}\right)$ have the same law. This terminates the proof, since we know that $\left(\mathcal{H}_{\partial F_{1}}, \ldots, \mathcal{H}_{\partial F_{n}}\right)$ and $\left(\mathcal{H}_{\ell_{1}}, \ldots, \mathcal{H}_{\ell_{2 g}}\right)$ are independent under the Yang-Mills measure. 


\subsection{Small scale structure of the Yang-Mills field}

\subsubsection{Extraction of a white noise}

In the first part of this chapter, we explained how the data of a white noise on $M$ and a bit more alea allows to reconstruct the Yang-Mills measure. We proceed now backwards: we try to extract a white noise from the Yang-Mills measure on a surface. In some sense, this amounts to compute the curvature of a Yang-Mills random connection.

As usual, $(M, \sigma)$ is given, as well as elements $x_{1}, \ldots, x_{p}$ of $G$, associated with the components of $\partial M$ and $x=x_{1} \ldots x_{p}$ or $x=1$ if $M$ has no boundary. We denote by $\left(\Omega_{M}, \mu_{M}\left(x_{1}, \ldots, x_{p}\right)\right)$ the space of the Yang-Mills measure on $M$.

In order to study the measure at small scale, we construct on $M$ a sequence of partitions in the following way. Let $\left(\Gamma_{n}\right)_{n \geq 0}$ be a sequence of graphs on $M$ such that $\Gamma_{n}$ has exactly $n$ faces denoted by $F_{j, n}, j=1, \ldots, n$. We assume that $\sigma\left(F_{j, n}\right)=\frac{\sigma(M)}{n}$ and also that the diameter of the faces decreases uniformly to 0 , i.e. that for any metric on $M, \sup _{j} \operatorname{diam}\left(F_{j, n}\right) \longrightarrow 0$. We fix an orientation of $M$ and assume that the boundaries of the $F_{j, n}$ 's are oriented with the usual convention. For each couple $(j, n)$ with $n \geq 0,1 \leq j \leq n$, we denote the random variable $\mathcal{H}_{\partial F_{j, n}}$ defined on $\left(\Omega_{M}, \mu_{M}\left(x_{1}, \ldots, x_{p}\right)\right)$ by $\mathcal{H}_{j, n}$ and see it as a $\mathbf{C}$-valued random variable, identifying $U(1)$ with $\{z \in \mathbf{C},|z|=1\}$.

For each $n \geq 0$, let $E_{n}$ denote the space of functions on $M$ constant on each face of $\Gamma_{n}$. Set $E_{\infty}=\cup_{n} E_{n}$. The assumption on the diameter of the faces $F_{j, n}$ imply that any continuous function on $M$ can be uniformly approximated by functions of $E_{\infty}$. Thus, $E_{\infty}$ is dense in $L^{1}(M, \sigma)$ and $L^{2}(M, \sigma)$ with their respective usual norms.

In order to define a kind of white noise, we will proceed as for the construction of the standard Wiener integral. We define a linear form $I_{n}$ on each $E_{n}$. Let $f_{n}$ be a function of $E_{n}$ and let $f_{j, n}$ be its value on $F_{j, n}$. We set

$$
I_{n}\left(f_{n}\right)=\frac{1}{i} \sum_{j=1}^{n} f_{j, n}\left(\mathcal{H}_{j, n}-1\right) .
$$

Theorem 3.2.1 Let $f$ be a square-integrable function on $M$ and $\left(f_{n}\right)_{n \geq 0}$ a sequence of functions converging to $f$ in $L^{2}$ norm and such that $f_{n} \in E_{n}$. Then the sequence $\left(I_{n}\left(f_{n}\right)\right)_{n \geq 0}$ converges in $L^{2}\left(\Omega_{M}, \mu_{M}\left(x_{1}, \ldots, x_{p}\right)\right)$ to a random variable $I(f)$ that does not depend on the choice of the sequence $\left(f_{n}\right)$. The law of this random variable can be described in the following way. Let $W_{f}^{0}$ be a centered gaussian random variable with variance $\|f\|_{L_{0}^{2}}^{2}=\left\|f-\frac{1}{\sigma(M)} \int_{M} f d \sigma\right\|_{L^{2}}^{2}$. Let $T$ be a $\mathcal{N}(0, \sigma(M))$ random variable conditioned to take its values in $\exp ^{-1}(x)$, independent of $W_{f}^{0}$. Then, the following identity holds in distribution:

$$
I(f) \stackrel{\text { law }}{=} W_{f}^{0}+\left(T+\frac{i}{2}\right) \frac{1}{\sigma(M)} \int_{M} f d \sigma .
$$

This proves in particular that the law of $I(f)$ does not depend on the choice of the orientation of $M$.

Proof: To prove this theorem, it is convenient to use the white noise realization of the YangMills measure. Let $(\Omega, P)$ be a probability space on which a pair $(W, T)$ is defined, consisting in a white noise $W$ and a random variable $T$ independent of $W$, whose law is that described in the 
theorem. We do not need the variables $U_{i}$, because we are only computing the holonomy along loops that are homologous to zero. Set

$$
Y_{j, n}=W\left(\mathbf{1}_{F_{j, n}}\right), \quad S_{n}=\sum_{j=1}^{n} Y_{j, n}, \quad X_{j, n}=Y_{j, n}-\frac{1}{n} S_{n} .
$$

We know by the theorem 3.1.11 that the law of the sequence $\left(I_{n}\left(f_{n}\right)\right)$ can be represented on $(\Omega, P)$ by

$$
\left(\frac{1}{i} \sum_{j=1}^{n} f_{j, n}\left(e^{i\left(X_{j, n}+\frac{T}{n}\right)}-1\right)\right)_{n \geq 0} .
$$

We will prove the theorem for this sequence. For this, we study the following Lagrange inequality:

$$
\begin{gathered}
\mid \sum_{j=1}^{n} f_{j, n}\left(e^{i\left(X_{j, n}+\frac{T}{n}\right)}-1\right)-i \sum_{j=1}^{n} f_{j, n}\left(X_{j, n}+\frac{T}{n}\right)+ \\
\frac{1}{2} \sum_{j=1}^{n} f_{j, n}\left(X_{j, n}+\frac{T}{n}\right)^{2} \mid \leq \\
\sum_{j=1}^{n}\left|f_{j, n}\right|\left|X_{j, n}+\frac{T}{n}\right|^{3}
\end{gathered}
$$

We will often use of the following lemma:

Lemma 3.2.2 For each positive integer $p$, there exists a constant $C_{p}$ such that

$$
E\left|X_{j, n}\right|^{p} \leq \frac{C_{p}}{n^{\frac{p}{2}}}
$$

Proof: This is just a consequence of the fact that a centered gaussian random variable $X$ of variance $t$ satisfies $E|X|^{p}=C_{p} t^{\frac{p}{2}}$ for come constant $C_{p}$ independent of $t$ and that $X_{j, n}$ has variance $\frac{\sigma(M)}{n}-\frac{\sigma(M)}{n^{2}}$.

We begin by showing that the right hand side term converges to zero in $L^{2}(\Omega, P) . \mathrm{y}$

$$
E\left|X_{j, n}+\frac{T}{n}\right|^{6} \leq \sum_{k=0}^{6}\left(\begin{array}{l}
6 \\
k
\end{array}\right) E\left|X_{j, n}\right|^{6-k} E \frac{|T|^{k}}{n^{k}} \leq \frac{K}{n^{3}},
$$

so that

$$
\begin{aligned}
E\left|\sum_{j=1}^{n}\right| f_{j, n}|| X_{j, n}+\left.\left.\frac{T}{n}\right|^{3}\right|^{2} & =\sum_{j, k=1}^{n}\left|f_{j, n} \| f_{k, n}\right| E\left|X_{j, n}+\frac{T}{n}\right|^{3}\left|X_{k, n}+\frac{T}{n}\right|^{3} \\
& \leq\left(\sup _{j=1}^{n} n\left\|\left(X_{j, n}+\frac{T}{n}\right)^{3}\right\|_{L^{2}}\right)^{2}\left(\sum_{j=1}^{n} \frac{\left|f_{j, n}\right|}{n}\right)^{2} \\
& \leq \frac{1}{n}\left\|f_{n}\right\|_{L^{1}}^{2} \underset{n \rightarrow \infty}{\longrightarrow} 0 .
\end{aligned}
$$

Now look at the second order term of the left hand side of (3.2). Let $m_{n}=\frac{1}{\sigma(M)} \int_{M} f_{n} d \sigma$ denote the mean of $f_{n}$ and $f_{n}^{0}=f_{n}-m_{n}$ denote its zero-mean part. We will use several times 
the fact that $\sum_{j} f_{j, n}^{0}=0$. We have

$$
\sum_{j=1}^{n} f_{j, n}\left(X_{j, n}+\frac{T}{n}\right)^{2}=\sum_{j=1}^{n} f_{j, n}^{0}\left(X_{j, n}+\frac{T}{n}\right)^{2}+m_{n} \sum_{j=1}^{n}\left(X_{j, n}+\frac{T}{n}\right)^{2} .
$$

Let us study the first term of this decomposition. In all estimations, $C$ denotes a constant, i.e. a number that depends neither on $j$ nor on $n$. It may denote different constants at different lines.

$$
\sum_{j=1}^{n} f_{j, n}^{0}\left(X_{j, n}+\frac{T}{n}\right)^{2}=\sum_{j=1}^{n} f_{j, n}^{0} X_{j, n}^{2}+2 \sum_{j=1}^{n} \frac{f_{j, n}^{0}}{n} X_{j, n} T .
$$

The first term of the right hand side term can be written:

$$
\sum_{j=1}^{n} f_{j, n}^{0} X_{j, n}^{2}=\sum_{j=1}^{n} f_{j, n}^{0}\left(Y_{j, n}-\frac{1}{n} S_{n}\right)^{2}=\sum_{j=1}^{n} f_{j, n}^{0} Y_{j, n}^{2}-2 \sum_{j=1}^{n} \frac{f_{j, n}^{0}}{n} Y_{j, n} S_{n} .
$$

On one hand,

$$
E\left|\sum_{j=1}^{n} f_{j, n}^{0} Y_{j, n}^{2}\right|^{2}=E\left|\sum_{j=1}^{n} \frac{f_{j, n}^{0}}{n} n Y_{j, n}^{2}\right|^{2}=\sum_{j=1}^{n} \frac{\left|f_{j, n}^{0}\right|^{2}}{2^{2 n}} E\left|n Y_{j, n}^{2}\right|^{2} \leq \frac{C}{n}\left\|f_{n}^{0}\right\|_{L^{2}} \underset{n \rightarrow \infty}{\longrightarrow} 0,
$$

since $E\left|n Y_{j, n}\right|^{2}$ depends neither on $j$ nor on $n$.

On the other hand,

$$
\left\|Y_{j, n} S_{n}\right\|_{L^{2}}^{2}=E\left(Y_{j, n}^{2} S_{n}^{2}\right) \leq n\left(E Y_{j, n}^{2}\right)^{2}+E Y_{j, n}^{4} \leq \frac{C}{n}
$$

implies

$$
\left\|\sum_{j=1}^{n} \frac{f_{j, n}^{0}}{n} Y_{j, n} S_{n}\right\|_{L^{2}} \leq \sum_{j=1}^{n} \frac{\left|f_{j, n}^{0}\right|}{n}\left\|Y_{j, n} S_{n}\right\|_{L^{2}} \leq \frac{C}{2^{\frac{n}{2}}}\left\|f_{n}^{0}\right\|_{L^{1}} \underset{n \rightarrow \infty}{\longrightarrow} 0 .
$$

We proved that the first term of the r.h.s. of (3.4) tends to 0 . To study the second one, note that

so that

$$
\left\|X_{j, n} T\right\|_{L^{2}}^{2}=E X_{j, n}^{2} E T^{2} \leq \frac{C}{n}
$$

$$
\left\|\sum_{j=1}^{n} \frac{f_{j, n}^{0}}{n} X_{j, n} T\right\|_{L^{2}} \leq \sum_{j=1}^{n} \frac{\left|f_{j, n}^{0}\right|}{n}\left\|X_{j, n} T\right\|_{L^{2}} \leq \frac{C}{\sqrt{n}}\left\|f_{j, n}^{0}\right\|_{L^{1}} \underset{n \rightarrow \infty}{\longrightarrow} 0 .
$$

We proved that the zero-mean part of $f_{n}$ does not contribute to the second order term. Let us study the last term of (3.3).

$$
m_{n} \sum_{j=1}^{n}\left(X_{j, n}+\frac{T}{n}\right)^{2}=m_{n} \sum_{j=1}^{n} X_{j, n}^{2}+\frac{m_{n} T}{2^{n-1}} \sum_{j=1}^{n} X_{j, n}+\frac{m_{n}}{n} T^{2} .
$$

We have $\sum_{j} X_{j, n}=0$ a.s. and $\frac{m_{n}}{n} T^{2} \longrightarrow 0$ a.s. . It remains

$$
m_{n} \sum_{j=1}^{n} X_{j, n}^{2}=m_{n} \sum_{j=1}^{n} Y_{j, n}^{2}+\frac{m_{n}}{n} S_{n}^{2}-\frac{m_{n}}{2^{n-1}} S_{n}^{2}
$$


Since the law of $S_{n}^{2}$ does not depend on $n$, the two last terms tend to zero. In order to determine the limit of the first one, we compute

$$
\begin{aligned}
E\left|\sum_{j=1}^{n} Y_{j, n}^{2}-\frac{1}{n}\right|^{2}=\sum_{j=1}^{n} E\left|Y_{j, n}^{2}-\frac{1}{n}\right|^{2} & =\sum_{j=1}^{n} E\left(Y_{j, n}^{4}+\frac{1}{n^{2}}-\frac{2 Y_{j, n}^{2}}{n}\right) \\
& =\sum_{j=1}^{n} \frac{C}{n^{2}}+\frac{1}{n^{2}}-\frac{2}{n^{2}} \leq \frac{C}{n^{2}} .
\end{aligned}
$$

Thus,

$$
m_{n} \sum_{j=1}^{n} Y_{j, n}^{2}=\sum_{j=1}^{n}\left(Y_{j, n}^{2}-\frac{1}{n}\right)+m_{n} \underset{n \rightarrow \infty}{\stackrel{L^{2}}{\longrightarrow}} \lim _{n \rightarrow \infty} m_{n}=\frac{1}{\sigma(M)} \int_{M} f d \sigma .
$$

We are done with the second order term. We finish the proof by studying the first order one.

$$
\begin{aligned}
& \sum_{j=1}^{n} f_{j, n}\left(X_{j, n}+\frac{T}{n}\right)=\sum_{j=1}^{n} f^{0} j, n X_{j, n}+m_{n} T \\
& =m_{n} T+\sum_{j=1}^{n} f_{j, n}^{0} Y_{j, n} \\
& =m_{n} T+W\left(\sum_{j=1}^{n} f_{j, n}^{0} \mathbf{1}_{F_{j, n}}\right) \\
& =W\left(f_{n}^{0}\right)+m_{n} T \underset{n \rightarrow \infty}{\stackrel{L^{2}}{\longrightarrow}} W\left(f^{0}\right)+\frac{T}{\sigma(M)} \int_{M} f d \sigma .
\end{aligned}
$$

We have proved that

$$
\frac{1}{i} \sum_{j=1}^{n} f_{j, n}\left(e^{i\left(X_{j, n}+\frac{T}{n}\right)}-1\right) \underset{n \rightarrow \infty}{\stackrel{L^{2}}{\longrightarrow}} W\left(f^{0}\right)+\left(T+\frac{i}{2}\right) \frac{1}{\sigma(M)} \int_{M} f d \sigma .
$$

This limit does not depend on the choice of the sequence $\left(f_{n}\right)$. Thus the sequence $\left(I_{n}\left(f_{n}\right)\right)$ converges also to a limit $I(f)$ that does not depend on the choice of $\left(f_{n}\right)$ and whose law is the law announced in the theorem.

\subsubsection{Meaning of the variable $T$}

As a conclusion for this chapter, we will spend a few lines to suggest a geometric interpretation for the variable $T$, whose meaning could seem to be quite mysterious.

In a deterministic setting with a smooth connection $\omega$, a construction similar to that of the map $f \mapsto I(f)$ would have given the map:

$$
f \mapsto \int_{M} f F(\omega)
$$

where $F(\omega)$ is the curvature 2-form of $\omega$. As long as we consider zero-mean functions, the comparison between this formula and (3.2) is in agreement with the heuristic principle saying 
that the curvature of a Yang-Mills random connection is a white noise, as explained in the introduction.

If we take the function $f$ identically equal to 1 in the deterministic setting, we get the total curvature $\int_{M} F(\omega)$ of the fiber bundle $P$ on which $\omega$ lives. This quantity is well known to be independent of $\omega$ and to be a topological invariant of $P$, namely its first Chern class. The probabilistic counterpart of this total curvature seems then to be $I(1)=T$, droping out the imaginary part. This discussion becomes really meaningful when $M$ is closed, because $P$ is not necessarily trivial. We mentioned at the beginning of the discretization procedure in section 1.3 that we had lost any topological information about the structure of $P$. If we compute the "random Chern class" of $P$ at the end of the construction, we find a weighted sum of all possible Chern classes, with the smallest weights for the most complicated types of bundles. This was already suggested by Witten Wi].

On the other hand, we can change our point of view in the following way: we have an expression of the random holonomy which depends explicitely on the Chern class of $P$. So if we replace $T$ by a deterministic multiple of $2 \pi$ in the definition 3.1.1, we are able to construct a random holonomy consistent with any prescribed type of bundle $P$.

\subsection{Square-integrability of the double-layer potential}

In this section, we prove the theorem 1.9.11. We claim that it is enough to prove the theorem on closed surfaces. Indeed, we did not use the square-integrability of the double-layer potential to prove the results 3.1.4 and 3.1.5, which show that the result on a surface with boundary can be deduced from the result on a minimal closure of this surface. Thus, we assume that $M$ is closed.

Proposition 3.3.1 There exists $R_{F}>0$ such that for all embedded path $c \in P M$ such that $\ell(c)<\frac{1}{4} R_{F}$, the double layer potential $u_{c}$ of $c$ is in $L^{\infty}(M)$.

This proposition implies obviously the theorem. It implies even more, namely that the double layer potential of any path is in $L^{\infty}$.

Proof. The proof relies on three facts. The first one is that we know the divergence near the diagonal of the Green function in an open subset of $\mathbf{R}^{2}$. The second one is that, according to a classical theorem due to Gauss [Ch], any metric on $M$ is locally conformally flat. The third point is that the Green function is conformally invariant.

Since $M$ is compact, the second remark implies that there exists a radius $R_{F}$ such that any geodesic ball of $M$ of radius smaller than $R_{F}$ is conformally flat. Let us choose an embedded path $c$ of length smaller than $R_{F} / 4$. For each $r>0$, we denote by $B_{r}$ the ball $B(c(0), r)$. Since $\ell(c)$ is smaller than $R_{F} / 4, c$ is contained in $B_{\frac{1}{2} R_{F}}$. Since the Green function $G$ is smooth outside the diagonal, $u_{c}$ is smooth outside $B_{\frac{1}{2} R_{F}}$. It is enough now to prove that it is bounded on $B_{\frac{3}{4} R_{F}}$ for example. Set $r=\frac{3}{4} R_{F}$.

The values of $u_{c}$ inside $\bar{B}_{r}$ depend only on the restriction of $G$ to $\bar{B}_{r} \times \bar{B}_{r}$. On this set, $G$ satisfies $\Delta G_{x}=\delta_{x}-\frac{1}{\sigma(M)}$. Our idea is to substract smooth functions to $G$ until we get something easier to compute than $G$ itself. Denote by $G^{0}$ the solution of:

$$
\left\{\begin{array}{l}
\Delta G_{x}^{0}=-\frac{1}{\sigma(M)} \\
G_{x}^{0}(y)=G_{x}(y) \quad \forall y \in \partial B_{r} .
\end{array}\right.
$$


It is a smooth function inside $B_{r}$. The function $G^{1}$ defined by $G^{1}=G-G^{0}$ satisfies

$$
\left\{\begin{array}{l}
\Delta G_{x}^{1}=\delta_{x} \\
G_{x}^{1}(y)=0 \quad \forall y \in \partial B_{r}
\end{array}\right.
$$

So, $G^{1}$ is the fundamental solution of $\Delta$ inside $B_{r}$ with Dirichlet boundary conditions. We can decompose $u_{c}(x)$ for any $x$ in $B_{r}$ according to:

$$
u_{c}(x)=\int_{c} * d G_{x}^{0}+\int_{c} * d G_{x}^{1}=u_{c}^{0}(x)+u_{c}^{1}(x) .
$$

The first term is smooth and we are led to study the second. This is where conformally flat coordinates are useful: we choose a local chart $\varphi: U \longrightarrow B_{r}$, where $U$ is an open subset of $\mathbf{R}^{2}$, such that the pull-back of the metric $g$ of $M$ by $\varphi$ is conformally equivalent to $d x^{2}+d y^{2}$ on $U$. The point is that $\varphi^{*} G^{1}$ is not only the fundamental solution of $\Delta_{\varphi^{*} g}$ with respect to the measure induced by $\varphi^{*} g$, but also the fundamental solution of $\Delta_{0}=\partial_{x}^{2}+\partial_{y}^{2}$ with respect to the flat metric on $U$, by conformal invariance of the Green function. This tells us that $\varphi^{*} G^{1}$ diverges like $\frac{1}{4 \pi} \log d(\cdot, \cdot)$ on the diagonal. In other words, there exists a smooth function $G^{2}$ on $B_{r} \times B_{r}$ such that $\varphi^{*} G^{1}=\frac{1}{4 \pi} \log d(\cdot, \cdot)+\varphi^{*} G^{2}$.

What we want is to prove that $u_{c}^{1}(x)$ is bounded inside $B_{r}$. It is equivalent to prove that $u_{c}^{1} \circ \varphi$ is bounded on $U$. But for any $y \in U$,

$$
u_{c}^{1} \circ \varphi(y)=\int_{c} * d G_{\varphi(y)}^{1}=\int_{\varphi^{-1}(c)} * d\left(\varphi^{*} G_{y}^{1}\right) .
$$

Note that in this last term, the Hodge operator $*$ is that of the metric $\varphi^{*} g$. It is not the same operator as that of the flat metric on $U$. Fortunately, the fact that these two metrics are conformally equivalent implies that their Hodge operators are pointwise proportionnal, i.e. one is deduced from the other by the multiplication by a positive bounded smooth function. Thus, it is sufficient to prove that $y \mapsto \int_{\varphi^{-1}(c)} * d \varphi^{*} G_{y}^{1}$ is bounded, the Hodge operator being now that of the flat metric. As already noticed, we can remove a smooth part of $G^{1}$ and keep only the part

$$
\frac{1}{4 \pi} \int_{\varphi^{-1}(c)} * d \log d(y, \cdot)
$$

A short computation shows that $1 / 4 \pi * d \log d(y, \cdot)$ is nothing but $1 / 2 \pi$ times the angle form $d \theta$ of the polar coordinates centered at $y$. This allows us to estimate very easily the integral of this form along a path.

For example, we know that the integral of this form along a simple loop is bounded by 1 . On the other hand, it is obvious and easy to prove by a direct computation that the integral of this form along a straight segment is bounded by $1 / 2$.

Consider the path $\varphi^{-1}(c)$. It is injective, hence it is possible to transform it into a simple loop by concatenating it with a finite number of segments. So, we can make the function that we want to estimate to be bounded by adding to it a finite number of bounded functions. This gives the result. 



\section{Chapter 4}

\section{Small scale structure in the semi-simple case}

The theorem 3.1.11 shows that it is possible to construct the Yang-Mills measure in a short and quite pleasant way when $G=U(1)$, using a white noise on $M$ as main ingredient. Is it possible to do something similar in general? The works of Sengupta and Driver [Dr2, Se1, Se2] lead to an ambiguous answer to that question. Indeed, in these works, the authors have constructed random holonomies, starting from a Lie algebra-valued white noise on $M$. Nevertheless, the family of loops along which they are able to define the holonomy is strongly dependent of a particular choice of coordinates on $M$, as we explained in the introduction. We think that this is more than a simple technical problem. Although there might exist some generalization of the construction made in section 3.1, we will show that a white noise is probably not the right object to start with.

Our idea is the following. Was it possible to realize the random holonomy using a white noise, it would be possible to find a lot of information by looking at the random holonomy at small scale, i.e. along very small loops. For example, the theorem 3.2.1 basically says that when $G=U(1)$, almost all the information about the holonomy along homologically trivial loops is available at infinitesimally small scale. We prove that, when $G$ is semi-simple, there is no information at all available at infinitesimally small scale, at least when one looks at it in the same way that we did in the Abelian case.

\subsection{Statement of a zero-one law}

We begin by stating the main result. The surface is $(M, \sigma)$ as usual. We assume that $G$ is a compact connected semi-simple Lie group, for example $S U(2)$. We choose $x_{1}, \ldots, x_{p}$ in $G$ and consider the probability space $\left(\Omega_{M}, \mu_{M}\left(x_{1}, \ldots, x_{p}\right)\right)$.

Let $L$ be a simple loop on $M$ which is the boundary of an open set $D$ diffeomorphic to a disk. For each $n \geq 0$, consider a graph on $D$ which has exactly $n$ faces $F_{1, n}, \ldots, F_{n, n}$ such that

$\sigma\left(F_{i, n}\right)=\frac{\sigma(D)}{n}$ for each $i$. This is very similar to the situation described in the section 3.2.

Was $G$ Abelian, we would have the equality of cycles $L=\partial F_{1, n}+\ldots+\partial F_{n, n}$, provided orientations are well chosen. This would imply $\mathcal{H}_{L}=\mathcal{H}_{\partial F_{1, n}} \ldots \mathcal{H}_{\partial F_{n, n}}$ and for any function $f$ continuous on $G / \mathrm{Ad}=G$,

$$
E\left[f\left(\mathcal{H}_{L}\right) \mid \mathcal{H}_{\partial F_{1, n}}, \ldots, \mathcal{H}_{\partial F_{n, n}}\right]=f\left(\mathcal{H}_{L}\right) .
$$


When $G$ is semi-simple, the situtation is the opposite.

Theorem 4.1.1 For any function $f$ continuous on $G / \mathrm{Ad}$, the following convergence holds:

$$
E\left[f\left(\mathcal{H}_{L}\right) \mid \mathcal{H}_{\partial F_{1, n}}, \ldots, \mathcal{H}_{\partial F_{n, n}}\right] \underset{n \rightarrow \infty}{\stackrel{L^{2}}{\rightarrow}} \operatorname{Ef}\left(\mathcal{H}_{L}\right)
$$

\subsection{Proof of the zero-one law}

\subsubsection{Computation of the conditional expectation}

In this section, we will compute the conditional expectation appearing in the statement of the theorem, keeping $n$ fixed. We abbreviate $F_{i, n}$ in $F_{i}$.

For each $F_{i}$, consider a sequence $\left(L_{i, k}\right)_{k \geq 0}$ of simple loops whose image is inside the interior

of $F_{i}$ and such that $L_{i, k} \underset{k \rightarrow \infty}{\stackrel{d_{1}}{\longrightarrow}} \partial F_{i}$. The proposition 2.10 .8 shows that the following convergence holds in probability:

$$
\left(\mathcal{H}_{L_{1, k}}, \ldots, \mathcal{H}_{L_{n, k}}\right) \underset{k \rightarrow \infty}{\stackrel{P}{\longrightarrow}}\left(\mathcal{H}_{\partial F_{1}}, \ldots, \mathcal{H}_{\partial F_{n}}\right) .
$$

Let $f$ and $f_{1}$ be continuous functions on $G / \mathrm{Ad}$ and $(G / \mathrm{Ad})^{n}$ respectively. This convergence implies the following one:

$$
E\left[f\left(\mathcal{H}_{L}\right) f_{1}\left(\mathcal{H}_{\partial F_{1}}, \ldots, \mathcal{H}_{\partial F_{n}}\right)\right]=\lim _{k \rightarrow \infty} E\left[f\left(\mathcal{H}_{L}\right) f_{1}\left(\mathcal{H}_{L_{1, k}}, \ldots, \mathcal{H}_{L_{n, k}}\right)\right]
$$

We are led to the computation of the second expectation, keeping $k$ fixed. We abreviate temporarily $L_{i, k}$ by $L_{i}$.

We construct a particular graph on $M$ such that $L, L_{1}, \ldots, L_{n} \in \Gamma^{*}$ (see fig. 4.1). Outside $D$, it has only one face. Its support contains the components $N_{1}, \ldots, N_{p}$ of $\partial M$, paths $c_{1}, \ldots, c_{p}$ joining $L(0)$ to the $N_{i}$ 's and simple loops $a_{1}, \ldots, a_{g}, b_{1}, \ldots, b_{g}$ that represent a basis of the $H_{1}$ of a minimal closure of $M$.

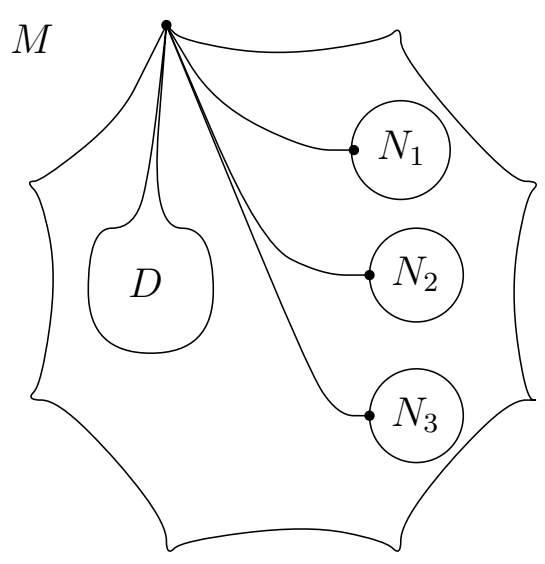

$D$

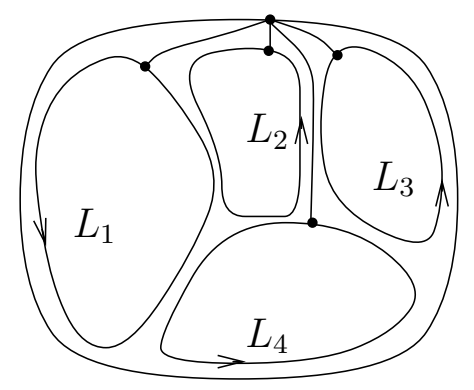

Figure 4.1: Aspect of $\Gamma$ outside and inside $D$.

The boundary of the unique face of $\Gamma$ outside $D$ is $L^{-1} c_{1} N_{1} c_{1}^{-1} \ldots c_{p} N_{p} c_{p}^{-1} \times \times\left[a_{1}, b_{1}\right] \ldots\left[a_{g}, b_{g}\right]$, where $[a, b]$ denotes the commutator $a b a^{-1} b^{-1}$ of $a$ and $b$. This notation is the same as that 
of joint conjugacy classes, but the context will always make our meaning clear. Inside $D$, the support of $\Gamma$ contains $n$ paths $d_{1}, \ldots, d_{n}$ joining $L(0)$ to the $L_{i}(0)$. These paths meet pairwise only at $L(0)$. Inside $D$, this graph has $n+1$ faces: $n$ whose boundaries are the $L_{i}$ 's and the last with boundary $L d_{n} L_{n}^{-1} d_{n}^{-1} \ldots d_{1} L_{1}^{-1} d_{1}^{-1}$. We denote by $t$ the surface of this last face and, for each $i=1, \ldots, n$, by $t_{i}$ the surface of the face bounded by $L_{i}$.

We compute $E\left[f\left(\mathcal{H}_{L}\right) f_{1}\left(\mathcal{H}_{L_{1}}, \ldots, \mathcal{H}_{L_{n}}\right)\right]$ in this graph. It is equal to

$$
\begin{aligned}
& \frac{1}{Z\left(x_{1}, \ldots, x_{p}\right)} \int_{G^{\Gamma}} f\left(\left[h_{L}\right]\right) f_{1}\left(\left[h_{L_{1}}\right], \ldots,\left[h_{L_{n}}\right]\right) p_{t_{1}}\left(h_{L_{1}}\right) \ldots p_{t_{n}}\left(h_{L_{n}}\right) \\
& p_{t}\left(h_{d_{1}}^{-1} h_{L_{1}}^{-1} h_{d_{1}} \ldots h_{d_{n}}^{-1} h_{L_{n}}^{-1} h_{d_{n}} h_{L}\right) \\
& p_{\sigma\left(D^{c}\right)}\left(\left[h_{b_{g}}^{-1}, h_{a_{g}}^{-1}\right] \ldots\left[h_{b_{1}}^{-1}, h_{a_{1}}^{-1}\right] h_{c_{p}}^{-1} h_{N_{p}} h_{c_{p}} \ldots h_{c_{1}}^{-1} h_{N_{1}} h_{c_{1}} h_{L}^{-1}\right) d \nu_{x_{1}} \ldots d \nu_{x_{p}} d g^{\prime} \\
& =\frac{1}{Z\left(x_{1}, \ldots, x_{p}\right)} \int_{G^{2(g+n)+p+1}} f([g]) f_{1}\left(\left[g_{1}\right], \ldots,\left[g_{n}\right]\right) p_{t_{1}}\left(g_{1}\right) \ldots p_{t_{n}}\left(g_{n}\right) \\
& p_{t}\left(y_{1}^{-1} g_{1}^{-1} y_{1} \ldots y_{n}^{-1} g_{n}^{-1} y_{n} g\right) p_{\sigma\left(D^{c}\right)}\left(\left[a_{g}, b_{g}\right] \ldots\left[a_{1}, b_{1}\right] z_{p}^{-1} x_{p} z_{p} \ldots z_{1}^{-1} x_{1} z_{1} g^{-1}\right) \\
& \quad d g d g_{1} \ldots d g_{n} d y_{1} \ldots d y_{n} d z_{1} \ldots d z_{p} d a_{1} \ldots d a_{g} d b_{1} \ldots d b_{g} .
\end{aligned}
$$

We used the fact that under $\nu_{x_{1}} \ldots \nu_{x_{p}} d g^{\prime}, h_{N_{i}}=x_{i}$ a.s. and $h_{L}$ and all $h_{L_{i}}, h_{a_{i}}, h_{b_{i}}, h_{c_{i}}, h_{d_{i}}$ are uniform and independent. When $k$ tends to infinity, each $t_{i}$ tends to $\frac{\sigma(D)}{n}$ and $t$ tends to zero. So, according to 1.5.1, we can drop $g$ and replace it by $y_{n}^{-1} g_{n} y_{n} \ldots y_{1}^{-1} g_{1} y_{1}$. This terminates the proof of the following proposition:

Proposition 4.2.1 The following equality holds:

$$
\begin{aligned}
& E\left[f\left(\mathcal{H}_{L}\right) \mid \mathcal{H}_{\partial F_{1, n}}, \ldots, \mathcal{H}_{\partial F_{n, n}}\right]= \\
& \left(\int p_{\sigma\left(D^{c}\right)}\left(\left[a_{g}, b_{g}\right] \ldots\left[a_{1}, b_{1}\right] z_{p}^{-1} x_{p} z_{p} \ldots z_{1}^{-1} x_{1} z_{1} y_{1}^{-1} K_{1} y_{1} \ldots y_{n}^{-1} K_{n} y_{n}\right) d y_{i} d z_{i} d a_{i} d b_{i}\right)^{-1} \cdot \\
& \quad \int f\left(y_{n}^{-1} K_{n} y_{n} \ldots y_{1}^{-1} K_{1} y_{1}\right) \\
& p_{\sigma\left(D^{c}\right)}\left(\left[a_{g}, b_{g}\right] \ldots\left[a_{1}, b_{1}\right] z_{p}^{-1} x_{p} z_{p} \ldots z_{1}^{-1} x_{1} z_{1} y_{1}^{-1} K_{1}^{-1} y_{1} \ldots y_{n}^{-1} K_{n}^{-1} y_{n}\right) d y_{i} d z_{i} d a_{i} d b_{i}
\end{aligned}
$$

where $K_{1}, \ldots, K_{n}$ are arbitrary representatives of $\mathcal{H}_{\partial F_{1, n}}, \ldots, \mathcal{H}_{\partial F_{n, n}}$.

Since we are dealing with functions on $G / \mathrm{Ad}$, it is natural to use the theory of characters on $G$. We give a very short account of the results that we will use. For a detailed presentation of the subject, see for example [B, $[\mathrm{Si}]$.

\subsubsection{Characters of a semi-simple Lie group}

A representation of $G$ is a smooth morphism of groups $\rho$ from $G$ into the linear group of some $\mathbf{C}^{n}$. The integer $n$ is the dimension of $\rho$. Since $G$ is compact, we may always assume that $\rho(G)$ is included in the unitary group. A representation is said to be irreducible if there are no subspaces of $\mathbf{C}^{n}$ invariant by all $\rho(g)$ except $\mathbf{C}^{n}$ and 0 . Two representations $\rho_{1}$ and $\rho_{2}$ of same dimension $n$ are said to be equivalent if there exists a linear isomorphism $\phi$ of $\mathbf{C}^{n}$ such that $\rho_{1} \circ \phi=\phi \circ \rho_{2}$. . The character of $\rho$ is the $\mathbf{C}$-valued function $\chi_{\rho}$ defined on $G$ by $\chi_{\rho}(g)=\operatorname{tr} \rho(g)$. Two equivalent representations have the same character. In fact, this is also a sufficient condition of equivalence. The usual properties of the trace imply that it is a central 
function on $G$, that is, $\chi_{\rho}(g)$ depends only on the conjugacy class of $g$. Since all representations are unitary, the relation $\chi_{\rho}\left(g^{-1}\right)=\chi_{\rho}(g)^{*}$ holds, the star denoting the complex conjugation. Note that $\chi_{\rho}(1)=\operatorname{dim} \rho$. The main theorem is the following:

Theorem 4.2.2 (Peter-Weyl theorem) The set of characters of all equivalence classes of irreducible representations of $G$ is an orthonormal basis of the space of central square-integrable functions on $(G, d g)$. Moreover, the algebra generated by this set is dense in the set of continuous C-valued central functions on $G$ endowed with the uniform norm.

According to this theorem, any continuous function can be approximated by linear combinations of products of characters. But it is a fact that such combinations can always be written as linear combinations of characters of irreducible representations. Thus it is sufficient to prove our theorem when $f$ is the character of an irreducible representation.

Characters satisfy orthogonality relations that give rise to useful formulas. We will mainly use two of them.

Proposition 4.2.3 For any $x, y, z \in G$ and for any irreducible representation $\alpha$ of $G$,

$$
\begin{aligned}
\int_{G} \chi_{\alpha}\left(x y x^{-1} z\right) d x & =\frac{1}{\operatorname{dim} \alpha} \chi_{\alpha}(x) \chi_{\alpha}(y), \\
\int_{G^{2}} \chi_{\alpha}([a, b] x) d a d b & =\frac{1}{(\operatorname{dim} \alpha)^{2}} \chi_{\alpha}(x) .
\end{aligned}
$$

Let us endow $G$ with its biinvariant metric normalized to have total volume equal to 1 . This metric gives rise to a Laplace operator on $G$. A remarkable property of the characters is that they are eigenfunctions for this operator. More precisely, for any irreducible representation $\alpha$, there exists a positive real number $c_{2}(\alpha)$ such that

$$
\Delta \chi_{\alpha}=-c_{2}(\alpha) \chi_{\alpha}
$$

A nice application of these properties is the computation of the character expansion of the heat kernel on $G$. Let us denote by $\widehat{G}$ the set of classes of irreducible representations of $G$.

Proposition 4.2.4 The following equality holds in $L^{2}(G, d g)$ and also pointwise on $G$ :

$$
p_{t}=\sum_{\beta \in \widehat{G}} \operatorname{dim} \beta e^{-\frac{c_{2}(\beta)}{2} t} \chi_{\beta} .
$$

Proof. We first prove the $L^{2}$ convergence. For any $t>0$, the function $p_{t}$ is a central $L^{2}$ function on $G$. Thus it admits a decomposition

$$
p_{t}=\sum_{\beta \in \widehat{G}} C_{\beta}(t) \chi_{\beta} .
$$

The differential equation $\left(\frac{1}{2} \Delta-\partial_{t}\right) p_{t}=0$ implies that $\left(\frac{1}{2} c_{2}(\beta)+\partial_{t}\right) C_{\beta}(t)=0$. Thus, $C_{\beta}(t)=$ $C_{\beta}(0) e^{-c_{2}(\beta) \frac{t}{2}}$. The constants $C_{\beta}(0)$ are determined using the fact that $p_{t}$ tends to $\delta_{1}$ as $t$ tends to 0 . It is easily checked that $C_{\beta}(0)=\operatorname{dim} \beta$ is a convenient choice. Formally, it amounts to check that $\sum_{\beta} \operatorname{dim} \beta \chi_{\beta}=\delta_{1}$.

To see that the convergence holds pointwise, note that the expansion of $p_{t}$ is a series of continuous functions that converges normally. Since $p_{t}$ is continuous, it is not only the sum of this series in the $L^{2}$ sense, but also in the sense of the uniform convergence. 


\subsubsection{Character computations}

We go back to the big expression obtained in proposition 4.2.1. From now on, we fix an irreducible representation $\alpha$ and put $f=\chi_{\alpha}$. We compute the numerator $N_{n}$ of the conditional expectation. The computations using characters presented in this section and the next one are very close to those done by Witten in Wi], when he expands explicitely partition functions in order to compute the symplectic volume of the moduli space of flat connections.

We begin by developing the heat kernel $p_{\sigma\left(D^{c}\right)}$ using proposition 4.2.4. We get a sum over $\beta$ of integrals of $\chi_{\alpha}$ of something times $\chi_{\beta}$ of something else. We integrate over the variables that appear only as arguments of $\chi_{\beta}$, first $a_{1}$ and $b_{1}$, and so on until $a_{g}$ and $b_{g}$, using (4.2). Each integration against $a_{i}$ and $b_{i}$ produces a factor $\frac{1}{(\operatorname{dim} \beta)^{2}}$. Then we integrate against $z_{1}, \ldots, z_{n}$ using (4.1). Each integration gives a factor $\chi_{\beta}\left(x_{i}\right) / \operatorname{dim} \beta$. At this stage, the arguments of $\chi_{\alpha}$ and $\chi_{\beta}$ are inverse of each other. Using the relation $\chi_{\beta}\left(g^{-1}\right)=\chi_{\beta}^{*}(g)$, we obtain:

$$
\begin{aligned}
& N_{n}=\sum_{\beta \in \widehat{G}}(\operatorname{dim} \beta)^{1-2 g-p} e^{-\frac{c_{2}(\beta)}{2} \sigma\left(D^{c}\right)} \prod_{i=1}^{p} \chi_{\beta}\left(x_{i}\right) \cdot \\
& \int_{G^{n}} \chi_{\alpha} \chi_{\beta}^{*}\left(y_{n}^{-1} K_{n} y_{n} \ldots y_{1}^{-1} K_{1} y_{1}\right) d y_{1} \ldots d y_{n} .
\end{aligned}
$$

We use the formal expansion of the central function $\chi_{\alpha} \chi_{\beta}^{*}=\sum_{\gamma \in \widehat{G}}\left(\chi_{\alpha} \chi_{\beta}^{*}, \chi_{\gamma}\right)_{L^{2}} \chi_{\gamma}$. We have now a sum over $\beta$ and $\gamma$ but only $\chi_{\gamma}$ under the integral. We integrate against $y_{1}, \ldots, y_{n}$ using (4.1). Note that the factor $\frac{1}{\operatorname{dim} \gamma}$ produced by the last integration cancels out with the remaining $\chi_{\gamma}(1)$. We find

$$
\begin{array}{r}
N_{n}=\sum_{\beta, \gamma \in \widehat{G}}(\operatorname{dim} \beta)^{1-2 g-p}(\operatorname{dim} \gamma)^{-(n-1)} e^{-\frac{c_{2}(\beta)}{2} \sigma\left(D^{c}\right)}\left(\chi_{\alpha} \chi_{\beta}^{*}, \chi_{\gamma}\right)_{L^{2}} \\
\prod_{i=1}^{p} \chi_{\beta}\left(x_{i}\right) \prod_{i=1}^{n} \chi_{\gamma}\left(K_{n}\right),
\end{array}
$$

with $\left(\chi_{\alpha} \chi_{\beta}^{*}, \chi_{\gamma}\right)_{L^{2}}=\int_{G} \chi_{\alpha}(g) \chi_{\beta}^{*}(g) \chi_{\gamma}^{*}(g) d g=\left(\chi_{\alpha}, \chi_{\beta} \chi_{\gamma}\right)_{L^{2}}$.

A similar and simpler computation leads to the following expression for the denominator $D_{n}$ of 4.2.1:

$$
D_{n}=\sum_{\beta \in \widehat{G}}(\operatorname{dim} \beta)^{2-2 g-p-n} e^{-\frac{c_{2}(\beta)}{2} \sigma\left(D^{c}\right)} \prod_{i=1}^{p} \chi_{\beta}\left(x_{p}\right) \prod_{i=1}^{n} \chi_{\beta}\left(K_{i}\right) .
$$

Remark that it is equivalent to evaluate a character at $K_{i}$ or on $\mathcal{H}_{\partial F_{i}}$. In order to prove the convergence, we need to know the asymptotic behaviour of an expression like

$$
\frac{1}{(\operatorname{dim} \beta)^{n-1}} \prod_{i=1}^{n} \chi_{\beta}\left(\mathcal{H}_{\partial F_{i}}\right) .
$$

The following result will be proved in the next section:

Proposition 4.2.5 For any $\beta \in \widehat{G}$, the following convergence holds:

$$
\frac{1}{(\operatorname{dim} \beta)^{n-1}} \prod_{i=1}^{n} \chi_{\beta}\left(\mathcal{H}_{\partial F_{i}}\right) \underset{n \rightarrow \infty}{\stackrel{L^{2}}{\longrightarrow}} \operatorname{dim} \beta e^{-\frac{c_{2}(\beta)}{2} \sigma(D)} .
$$


The inequality $\left|\chi_{\beta}(g)\right| \leq \operatorname{dim} \beta$ shows that the sequence is uniformly bounded by $\operatorname{dim} \beta$. This allows us to permute this convergence with the summation over $\beta$. We get the following $L^{2}$-limit for the numerator:

$$
\sum_{\beta, \gamma \in \widehat{G}}(\operatorname{dim} \beta)^{1-2 g-p} e^{-\frac{c_{2}(\beta)}{2} \sigma\left(D^{c}\right)} \prod_{i=1}^{p} \chi_{\beta}\left(x_{p}\right)\left(\chi_{\alpha}, \chi_{\beta} \chi_{\gamma}\right)_{L^{2}} \operatorname{dim} \gamma e^{-\frac{c_{2}(\gamma)}{2} \sigma(D)} .
$$

By the same kind of arguments that we used to derive the expressions of $D_{n}$ and $N_{n}$, it is easy to check that this expression is equal to the following:

$$
\int_{G^{p+2 g+1}} \chi_{\alpha}(g) p_{\sigma(D)}\left(g^{-1}\right) p_{\sigma\left(D^{c}\right)}\left(g^{-1} z_{1} x_{1} z_{1}^{-1} \ldots z_{p} x_{p} z_{p}^{-1}\left[a_{1}, b_{1}\right] \ldots\left[a_{g}, b_{g}\right]\right) d g d z_{i} d a_{i} d b_{i}
$$

For the denominator, we find the following $L^{2}$-limit:

$$
\sum_{\beta \in \widehat{G}}(\operatorname{dim} \beta)^{2-2 g-p} e^{-\frac{c_{2}(\beta)}{2}\left(\sigma\left(D^{c}\right)+\sigma(D)\right)} \prod_{i=1}^{p} \chi_{\beta}\left(x_{p}\right),
$$

which is equal to:

$$
\int_{G^{p+2 g}} p_{\sigma(M)}\left(z_{1} x_{1} z_{1}^{-1} \ldots z_{p} x_{p} z_{p}^{-1}\left[a_{1}, b_{1}\right] \ldots\left[a_{g}, b_{g}\right]\right) d z_{i} d a_{i} d b_{i}=Z_{M}\left(x_{1}, \ldots, x_{p}\right) .
$$

Using the fact that $p_{\sigma(D)}\left(g^{-1}\right)=p_{\sigma(D)}(g)$, we see that the quotient of $(4.5)$ by (4.6) is equal to $E \chi_{\alpha}\left(\mathcal{H}_{L}\right)$. This proves the theorem, up to the proposition 4.2.5 that was admitted.

\subsubsection{Zero-one law on the plane}

In order to prove proposition 4.2.5, we begin with the following result, which can be seen as a reformulation of the zero-one law when the manifold $M$ is the plane $\mathbf{R}^{2}$.

Proposition 4.2.6 Let $\left(B_{t}^{n}\right)_{t \in \mathbf{R}_{+}, n \geq 0}$ be a sequence of independent Brownian motions on $G$. For any irreducible representation $\beta$ of $G$ and any positive real number $T$, the following convergence holds in distribution:

$$
\frac{1}{(\operatorname{dim} \beta)^{n-1}} \prod_{i=1}^{n} \chi_{\beta}\left(B_{\frac{T}{n}}^{i}\right) \underset{n \rightarrow \infty}{\stackrel{\operatorname{law}}{\longrightarrow}} \operatorname{dim} \beta e^{-\frac{c_{2}(\beta)}{2} T} .
$$

This result is really the center of the whole proof of the theorem. It is the place where the fact that $G$ is semi-simple will be used, in the following way. Given a representation $\rho$ of $G$, the differential at 1 of $\rho$ is a linear map from $T_{1} G$, the Lie algebra of $G$, into $\mathcal{L}\left(\mathbf{C}^{n}\right)$. The following statement is proved in Bourbaki (Lie, chap. I, $\S 6, \mathrm{~N}^{\circ} 2$, corollary of the th. 1) [B2]:

Proposition 4.2.7 Let $G$ be a semi-simple group and $\rho$ a representation of $G$ of dimension $n$. Then

$$
d_{1} \rho\left(T_{1} G\right) \subset \mathfrak{s l}_{n}(\mathbf{C})
$$

where $\mathfrak{s l}_{n}(\mathbf{C})$ denotes the set of endomorphisms of $\mathbf{C}^{n}$ whose trace is equal to zero. 
Proofof proposition 1.2.6: We consider a Brownian motion $\left(B_{t}\right)$ on $G$ and study the process $\chi_{\alpha}\left(B_{t}\right)$. There is a convenient way to represent $\left(B_{t}\right)$, as a solution of a Stratonovich stochastic differential equation [IW]. Recall that the data of a biinvariant metric on $G$ is equivalent to that of a scalar product on the Lie algebra of $G$, invariant by adjunction. Let $\left(X_{1}, \ldots, X_{\operatorname{dim} G}\right)$ be a basis of $T_{1} G$ orthonormal for this scalar product. Each $X_{i}$ is seen as a left-invariant vector field on $G$. Let $W^{1}, \ldots, W^{\operatorname{dim} G}$ be independent real Brownian motions. Then the Brownian motion on $G$ satisfies:

$$
\left\{\begin{array}{l}
d B_{t}=\sum_{i=1}^{\operatorname{dim} G} X_{i} \circ d W_{t}^{i} \\
B_{0}=1
\end{array}\right.
$$

The meaning of this notation is that, for any continuous function $f$ on $G$,

$$
f\left(B_{t}\right)=f(1)+\sum_{i=1}^{\operatorname{dim} G} \int_{0}^{t} X_{i} f\left(B_{s}\right) d W_{s}^{i}+\frac{1}{2} \int_{0}^{t} \Delta f\left(B_{s}\right) d s .
$$

We apply this relation to $f=\chi_{\alpha}$. Using $\chi_{\alpha}(1)=\operatorname{dim} \alpha$ and $\Delta \chi_{\alpha}=-c_{2}(\alpha) \chi_{\alpha}$, it becomes:

$$
\begin{aligned}
\frac{\chi_{\alpha}\left(B_{t}\right)}{\operatorname{dim} \alpha}=1-\frac{c_{2}(\alpha) t}{2}+\frac{1}{\operatorname{dim} \alpha} \sum_{i=1}^{\operatorname{dim} G} & \int_{0}^{t} X_{i} \chi_{\alpha}\left(B_{s}\right) d W_{s}^{i} \\
& -\frac{c_{2}(\alpha)}{2 \operatorname{dim} \alpha} \int_{0}^{t}\left(\chi_{\alpha}\left(B_{s}\right)-\operatorname{dim} \alpha\right) d s .
\end{aligned}
$$

Set

$$
\begin{gathered}
Y_{t}=-\frac{c_{2}(\alpha)}{2 \operatorname{dim} \alpha} \int_{0}^{t}\left(\chi_{\alpha}\left(B_{s}\right)-\operatorname{dim} \alpha\right) d s \\
Z_{t}=\frac{1}{\operatorname{dim} \alpha} \sum_{i=1}^{\operatorname{dim} G} \int_{0}^{t} X_{i} \chi_{\alpha}\left(B_{s}\right) d W_{s}^{i}
\end{gathered}
$$

We keep the notation $\rho(g)$ for the biinvariant distance $d(1, g)$ when $g \in G$. This $\rho$ has nothing to do with a representation of $G$ !

For any $X \in T_{1} G$, we have:

$$
d_{1} \chi_{\alpha}(X)=\left.\frac{d}{d t}\right|_{t=0} \operatorname{tr} \alpha(\exp t X)=\operatorname{tr} d_{1} \alpha(X)=0,
$$

according to 4.2.7. Thus the differential of $\chi_{\alpha}$ at 1 is zero. This implies that $\left|\chi_{\alpha}(g)-\operatorname{dim} \alpha\right|=$ $O\left(\rho(g)^{2}\right)$ in a neighbourhood of 1. Using the lemma 1.8.3, we get:

$$
\begin{aligned}
E\left|Y_{t}\right|^{2} & =C E\left|\int_{0}^{t}\left(\chi_{\alpha}\left(B_{s}\right)-\operatorname{dim} \alpha\right) d s\right|^{2} \\
& \leq C t E \int_{0}^{t}\left|\chi_{\alpha}\left(B_{s}\right)-\operatorname{dim} \alpha\right|^{2} d s \\
& \leq C t E \int_{0}^{t} \rho\left(B_{s}\right)^{4} d s \\
& \leq C t \int_{0}^{t} s^{2} d s \\
& \leq C t^{4}
\end{aligned}
$$


For each $i=1, \ldots, \operatorname{dim} G$, the function $X_{i} \chi_{\alpha}$ is smooth and $X_{i} \chi_{\alpha}(1)=0$. Thus, $\left|X_{i} \chi_{\alpha}(g)\right|=$ $O(\rho(g))$ in a neighbourhood of 1 . Thus,

$$
\begin{aligned}
E\left|Z_{t}^{2}\right| & =C E\left|\sum_{i=1}^{\operatorname{dim} G} \int_{0}^{t} X_{i} \chi_{\alpha}\left(B_{s}\right) d W_{s}^{i}\right|^{2} \\
& =C \sum_{i=1}^{\operatorname{dim} G} E\left|\int_{0}^{t} X_{i} \chi_{\alpha}\left(B_{s}\right) d W_{s}^{i}\right|^{2} \\
& =C \sum_{i=1}^{\operatorname{dim} G} E \int_{0}^{t}\left|X_{i} \chi_{\alpha}\left(B_{s}\right)\right|^{2} d s \\
& \leq C \operatorname{dim} G \int_{0}^{t} E \rho\left(B_{s}\right)^{2} d s \\
& \leq C t^{2} .
\end{aligned}
$$

The preliminary study of the process $\chi_{\alpha}\left(B_{t}\right)$ is now finished. We consider a sequence $\left(B_{t}^{n}\right)$ as in the statement of 4.2 .6 , defined on a probability space $(\Omega, P)$. We look at the product

$$
X_{n}=\prod_{i=1}^{n}\left(1-\frac{c_{2}(\alpha) T}{2 n}+Z_{T / n}^{i}+Y_{T / n}^{i}\right)
$$

where the random variables with different exponents are independent. We would like to take the logarithm of this product. This requires some precautions. Set

$$
\Omega_{n}=\left\{\left|Z_{T / n}^{i}+Y_{T / n}^{i}\right|<\frac{1}{3} \forall i=1 \ldots n\right\}
$$

A Chebishev inequality gives

$$
P\left(\left|Z_{t}+Y_{t}\right|<\frac{1}{3}\right) \geq 1-9 E\left|Z_{t}+Y_{t}\right|^{2} \geq 1-C t^{2},
$$

implying

$$
P\left(\Omega_{n}\right) \geq\left(1-\frac{C T^{2}}{n^{2}}\right)^{n} \underset{n \rightarrow \infty}{\longrightarrow} 1 .
$$

We do not change any convergence in distribution on $\Omega$ if we replace $X_{n}$ by 1 outside $\Omega_{n}$. So we set

$$
\tilde{X}_{n}=X_{n} \mathbf{1}_{\Omega_{n}}+\mathbf{1}_{\Omega_{n}^{c}}
$$

Then $\log \widetilde{X}_{n}$ is well defined, $\log$ being the principal determination of the complex logarithm. In fact, we have more than that. If $n$ is such that $\frac{c_{2}(\alpha) T}{2 n}$ is smaller than $\frac{1}{6}$, then each factor of $\widetilde{X}_{n}$ is of the form $(1-z)$ with $|z|<\frac{1}{2}$. For such a $z$, we have $|\log (1-z)+z| \leq|z|^{2}$. Thus, the equality

$$
\log \left(\tilde{X}_{n}\right)=\mathbf{1}_{\Omega_{n}} \sum_{i=1}^{n} \log \left(1-\frac{c_{2}(\alpha) T}{2 n}+Z_{T / n}^{i}+Y_{T / n}^{i}\right)
$$


implies

$$
\begin{aligned}
\left|\log \widetilde{X}_{n}+\sum_{i=1}^{n}\left(\frac{c_{2}(\alpha) T}{2 n}-Z_{T / n}^{i}-Y_{T / n}^{i}\right)\right| & \leq \mathbf{1}_{\Omega_{n}} \sum_{i=1}^{n}\left|\frac{c_{2}(\alpha) T}{2 n}-Z_{T / n}^{i}-Y_{T / n}^{i}\right|^{2}+ \\
& \mathbf{1}_{\Omega_{n}^{c}}\left|\sum_{i=1}^{n} \frac{c_{2}(\alpha) T}{2 n}-Z_{T / n}^{i}-Y_{T / n}^{i}\right| .
\end{aligned}
$$

The last term tends to 0 in probability because $P\left(\Omega_{n}^{c}\right)$ tends to 0 . Using (4.8) and (4.9), we find

$$
E\left|\frac{c_{2}(\alpha) T}{2 n}-Z_{T / n}^{i}-Y_{T / n}^{i}\right|^{2} \leq \frac{C T^{2}}{n^{2}}
$$

so that the first term of the right hand side of (4.10) tends to 0 in $L^{1}$ norm.

Now let us study the left hand side. On one hand, (4.8) implies $\sum_{i} Y_{T / n}^{i} \stackrel{L^{1}}{\rightarrow} 0$, because

$$
E\left|\sum_{i=1}^{n} Y_{T / n}^{i}\right| \leq \sum_{i=1}^{n} E\left|Y_{T / n}^{i}\right| \leq \frac{C}{n} .
$$

On the other hand, using 4.9 and the fact that $E Z_{T / n}^{i}=0$, we also have $\sum_{i} Z_{T / n}^{i} \stackrel{L^{1}}{\rightarrow} 0$, because

$$
E\left|\sum_{i=1}^{n} Z_{T / n}^{i}\right|^{2}=\sum_{i=1}^{n} E\left|Z_{T / n}^{i}\right|^{2} \leq \frac{C}{n} .
$$

We deduce that $\log \tilde{X}_{n}$ converges in probability to $-\frac{c_{2}(\alpha) T}{2}$. This implies that $\tilde{X}_{n}$, and so $X_{n}$, converge in probability to $e^{-\frac{c_{2}(\alpha) T}{2}}$. Finally, we get

$$
\operatorname{dim} \alpha X_{n} \underset{n \rightarrow \infty}{\stackrel{\text { law }}{\longrightarrow}} \operatorname{dim} \alpha e^{-\frac{c_{2}(\alpha) T}{2}},
$$

proving the proposition.

The proof of the theorem is almost finished. It remains to prove that the proposition 4.2.6 implies the proposition 4.2.5.

Proofof 4.2.5: We set $T=\sigma(D)$. Let $F$ be a continuous function on $\mathbf{R}$.

$$
\begin{gathered}
E\left[F\left(\frac{1}{(\operatorname{dim} \beta)^{n-1}} \prod_{i=1}^{n} \chi_{\beta}\left(\mathcal{H}_{\partial F_{i}}\right)\right)\right]=\frac{1}{Z\left(x_{1}, \ldots, x_{p}\right)} \int F\left(\frac{1}{(\operatorname{dim} \beta)^{n-1}} \prod_{i=1}^{n} \chi_{\beta}\left(g_{i}\right)\right) \\
p_{\sigma\left(D^{c}\right)}\left(\left[a_{g}, b_{g}\right] \ldots\left[a_{1}, b_{1}\right] z_{p}^{-1} x_{p} z_{p} \ldots z_{1}^{-1} x_{1} z_{1} y_{1}^{-1} g_{1}^{-1} y_{1} \ldots y_{n}^{-1} g_{n}^{-1} y_{n}\right) \\
p_{T / n}\left(g_{1}\right) \ldots p_{T / n}\left(g_{n}\right) d g_{i} d a_{i} d b_{i} d y_{i} d z_{i} .
\end{gathered}
$$

We develop the heat kernel $p_{\sigma\left(D^{c}\right)}$ and integrate against all variables except $g_{1}, \ldots, g_{n}$. We find

$$
\begin{aligned}
\frac{1}{Z\left(x_{1}, \ldots, x_{p}\right)} \sum_{\gamma \in \widehat{G}}(\operatorname{dim} \gamma)^{1-2 g-p} e^{-\frac{c_{2}(\gamma)}{2} \sigma\left(D^{c}\right)} \prod_{i=1}^{p} \chi_{\gamma}\left(x_{p}\right) \\
\int_{G^{n}} F\left(\frac{1}{(\operatorname{dim} \beta)^{n-1}} \prod_{i=1}^{n} \chi_{\beta}\left(g_{i}\right)\right) \frac{1}{(\operatorname{dim} \gamma)^{n-1}} \prod_{i=1}^{n} \chi_{\gamma}\left(g_{i}\right) \\
p_{T / n}\left(g_{1}\right) \ldots p_{T / n}\left(g_{n}\right) d g_{1} \ldots d g_{n} .
\end{aligned}
$$


The proposition 4.2.6 says exactly that this last integral converges to

$$
F\left(\operatorname{dim} \beta e^{-\frac{c_{2}(\beta)}{2} \sigma(D)}\right) \operatorname{dim} \gamma e^{-\frac{c_{2}(\gamma)}{2} \sigma(D)} .
$$

Thus,

$$
\begin{aligned}
E & {\left[F\left(\frac{1}{(\operatorname{dim} \beta)^{n-1}} \prod_{i=1}^{n} \chi_{\beta}\left(\mathcal{H}_{\partial F_{i}}\right)\right)\right] \underset{n \rightarrow \infty}{\longrightarrow} \frac{1}{Z\left(x_{1}, \ldots, x_{p}\right)} \times } \\
& \sum_{\gamma \in \widehat{G}}(\operatorname{dim} \gamma)^{2-2 g-p} e^{-\frac{c_{2}(\gamma)}{2} \sigma(M)} \frac{1}{(\operatorname{dim} \gamma)^{p-1}} \prod_{i=1}^{p} \chi_{\gamma}\left(x_{i}\right) F\left(\operatorname{dim} \beta e^{-\frac{c_{2}(\beta)}{2} \sigma(D)}\right) \\
= & \frac{Z\left(x_{1}, \ldots, x_{p}\right)}{Z\left(x_{1}, \ldots, x_{p}\right)} F\left(\operatorname{dim} \beta e^{-\frac{c_{2}(\beta)}{2} \sigma(D)}\right) .
\end{aligned}
$$

This proves that the announced convergence holds in distribution. We already noted that the function $\chi_{\beta}$ is bounded on $G$ by $\operatorname{dim} \beta$. So, the sequence that we study is uniformly bounded by $\operatorname{dim} \beta$. The result follows, since the convergence in distribution of a uniformly bounded sequence to a deterministic limit implies its $L^{2}$ convergence. 


\section{Chapter 5}

\section{Surgery of surfaces}

\subsection{Markov property of the Yang-Mills field}

Consider a surface $M$ which is cut into two pieces $M_{1}$ and $M_{2}$. Our aim in the first part of this chapter is to understand the relationships between the Yang-Mills measures on $M_{1}, M_{2}$ and $M$. We already met a question of this kind when we constructed the random holonomy on a surface with boundary starting from that on a minimal closure of this surface and this led us to prove in a particular case the Markov property which is the object of the theorem 5.1.1.

Let $M_{1}$ and $M_{2}$ be two oriented surfaces with boundary such that $\partial M_{1}$ and $\partial M_{2}$ have at least $p$ boundary components, where $p>0$ is arbitrary. Pick $p$ components $N_{1}, \ldots, N_{p}$ on $\partial M_{1}$ and $p$ others $N_{1}^{\prime}, \ldots, N_{p}^{\prime}$ on $\partial M_{2}$. For each $i=1, \ldots, p$, consider an orientation-reversing diffeomorphism $\psi_{i}: N_{i} \longrightarrow N_{i}^{\prime}$ and call $M$ the result of the gluing of $M_{1}$ and $M_{2}$ along $\psi_{1}, \ldots, \psi_{p}$. Denote by $L_{1}, \ldots, L_{p} p$ loops on $M$ whose images are the components of the common boundary of $M_{1}$ and $M_{2}$, oriented as boundary components of $M_{1}$.

For the statement of the theorem 5.1.1, we consider the Yang-Mills measures on $M, M_{1}$ and $M_{2}$ measures on the spaces $\mathcal{F}(L M, G), \mathcal{F}\left(L M_{1}, G\right)$ and $\mathcal{F}\left(L M_{2}, G\right)$ endowed with the $\sigma$-algebras $\mathcal{A}, \mathcal{A}_{1}$ and $\mathcal{A}_{2}$ generated by the variables of the form $\mathcal{H}_{l_{1}, \ldots, l_{n}}$.

There are two natural sub- $\sigma$-algebras on the space $(\mathcal{F}(L M, G), \mathcal{A})$, namely $\widetilde{\mathcal{A}}_{i}=\sigma\left(\mathcal{H}_{l_{1}, \ldots, l_{n}}, l_{k} \in\right.$ $\left.L M_{i}\right), i=1,2$. For any $i=1,2$, a function $f_{i}$ on $\mathcal{F}\left(L M_{i}, G\right)$ gives rise to a function $\widetilde{f}_{i}$ on $\mathcal{F}(L M, G)$ and it is equivalent to say that $f_{i}$ is $\mathcal{A}_{i}$-measurable or to say that $\tilde{f}_{i}$ is $\widetilde{\mathcal{A}}_{i^{-}}$-measurable, so that we identify $\mathcal{A}_{i}$ and $\widetilde{\mathcal{A}}_{i}$, as well as $f_{i}$ and $\widetilde{f}_{i}$.

Theorem 5.1.1 The $\sigma$-algebras $\mathcal{A}_{1}$ and $\mathcal{A}_{2}$ are independent on $\mathcal{F}(L M, G)$ under $\mu_{M}$ conditionally to the random variable $\left(\mathcal{H}_{L_{1}}, \ldots, \mathcal{H}_{L_{p}}\right)$. Moreover, let $f_{1}$ and $f_{2}$ be two measurable functions on $\left(\mathcal{F}\left(L M_{1}, G\right), \mathcal{A}_{1}\right)$ and $\left(\mathcal{F}\left(L M_{2}, G\right), \mathcal{A}_{2}\right)$ respectively. Then the product $f_{1} f_{2}$ can be seen as a $\mathcal{A}$-measurable function on $\mathcal{F}(L M, G)$ and for any $t_{1}, \ldots, t_{p} \in G / \mathrm{Ad}$, the following equality holds:

$$
\mu_{M}\left(t_{1}, \ldots, t_{p}\right)\left(f_{1} f_{2}\right)=\mu_{M_{1}}\left(t_{1}, \ldots, t_{p}\right)\left(f_{1}\right) \mu_{M_{2}}\left(t_{1}^{-1}, \ldots, t_{p}^{-1}\right)\left(f_{2}\right) .
$$

Finally, these properties remain true if we condition further the Yang-Mills measures with respect to the random holonomy along some boundary components or some interior loops on $M_{1}$ or $M_{2}$.

This theorem says two things. It says that the random holonomy on $M_{1}$ is independent of that on $M_{2}$ conditionally to the holonomy along the common boundary of $M_{1}$ and $M_{2}$ and it 
also says that the restriction to $\mathcal{A}_{i}$ of the measure $\mu_{M}\left(t_{1}, \ldots, t_{p}\right)$ is equal via the identification mentioned above to the measure $\mu_{M_{i}}\left(t_{1}, \ldots, t_{p}\right)$, that is, the Yang-Mills measure on $M_{i}$.

We prove first a discrete result, which is due, in a slightly different form, to Becker and Sengupta $[\mathrm{BS}]$.

Proposition 5.1.2 Let $\Gamma$ be a graph on $M$. Let $\Gamma_{1}$ and $\Gamma_{2}$ be the graphs on $M_{1}$ and $M_{2}$ induced by $\Gamma$. Let $f$ be a continuous function on $G^{\Gamma_{1}} \times G^{\Gamma_{2}}$ invariant by both the gauge transformations on $\Gamma_{1}$ and $\Gamma_{2}$. Then $f$ gives rise to a gauge-invariant function on $G^{\Gamma}$, still denoted by $f$ and for any $x \in G^{p}$,

$$
\int_{G^{\Gamma_{1}} \times G^{\Gamma_{2}}} f d P^{\Gamma_{1}}\left(y_{1}, x\right) d P^{\Gamma_{2}}\left(x^{-1}, y_{2}\right)=\int_{G^{\Gamma}} f d P^{\Gamma}\left(y_{1}, x, y_{2}\right),
$$

where $y_{1}$ and $y_{2}$ represent the values of the holonomy imposed along the remaining components of $\partial M_{1}$ and $\partial M_{2}$ and possibly other loops inside $M_{1}$ and $M_{2}$.

Proof. By a standard density argument, it is enough to prove this proposition when $f$ is a product $f_{1} f_{2}$ of two gauge-invariant functions on $G^{\Gamma_{1}}$ and $G^{\Gamma_{2}}$. In order to shorten the expressions, we abbreviate expressions like $d \nu_{x_{1}} \ldots d \nu_{x_{p}}$ into $d \nu_{x}$ if $x=\left(x_{1}, \ldots, x_{p}\right)$. So, if $f=f_{1} f_{2}$,

$$
\begin{array}{r}
\int_{G^{\Gamma_{1}} \times G^{\Gamma_{2}}} f_{1} f_{2} d P^{\Gamma_{1}}\left(y_{1}, x\right) d P^{\Gamma_{2}}\left(x^{-1}, y_{2}\right)=\int_{G^{\Gamma_{1}}} f_{1} d P^{\Gamma_{1}}\left(y_{1}, x\right) \int_{G^{\Gamma_{2}}} f_{2} d P^{\Gamma_{2}}\left(x^{-1}, y_{2}\right) \\
=\frac{1}{Z_{M_{1}}\left(y_{1}, x\right)} \int_{G^{\Gamma_{1}}} f_{1} D^{\Gamma_{1}} d \nu_{y_{1}} d \nu_{x} d g^{\Gamma_{1}} \frac{1}{Z_{M_{2}}\left(x^{-1}, y_{2}\right)} \int_{G^{\Gamma_{2}}} f_{2} D^{\Gamma_{2}} d \nu_{y_{2}} d \nu_{x^{-1}} d g^{\prime \Gamma_{2}} .
\end{array}
$$

The two integrals in the last expression are very similar and it is enough to study the first one in order to study both of them. Denote by $\Gamma_{1, \partial}$ the graph on $\partial M_{1}$ induced by $\Gamma_{1}$. The measure $d \nu_{x}$ concerns precisely the variables associated with the edges of $\Gamma_{1, \partial}$. Thus,

$$
\int_{G^{\Gamma_{1}}} f_{1} D^{\Gamma_{1}} d \nu_{y_{1}} d \nu_{x} d g^{\prime \Gamma_{1}}=\int_{G^{\Gamma_{1, \partial}}} d \nu_{x} \int_{G^{\Gamma_{1} \backslash \Gamma_{1, \partial}}} f_{1} D^{\Gamma_{1}} d \nu_{y_{1}} d g^{\prime \Gamma_{1}}
$$

The function $I_{1}=\int_{G^{\Gamma_{1}} \backslash \Gamma_{1, \partial}} f_{1} D^{\Gamma_{1}} d \nu_{y_{1}} d g^{\prime \Gamma_{1}}$ is a function on $G^{\Gamma_{\partial, 1}}$ and we claim that it depends only on the values of the functions $\mathcal{H}_{L_{1}}, \ldots, \mathcal{H}_{L_{p}}$, which are also well defined on this space.

This depends on the fact that $f_{1} D^{\Gamma_{1}}$ is a gauge-invariant function on $G^{\Gamma_{1}}$ and on the biinvariance of the Haar measure. Indeed, consider a gauge transformation $j$ on $\Gamma_{1}$, which is equal to 1 , except on vertices located on $\Gamma_{1, \partial}$. This gauge transformation changes the value of the holonomies along the edges of $\Gamma_{1, \partial}$, and $j$ can be choosed in such a way that these holonomies take any prescribed values, provided the conjugacy classes of the holonomies along $L_{1}, \ldots, L_{p}$ remain unchanged. So, we just need to prove that the value of $I_{1}$ is preserved by the action of such a gauge transformation. But this action also affects the holonomy along the other edges that meet $\Gamma_{1, \partial}$, so that the gauge invariance of $f_{1}$ and $D^{\Gamma_{1}}$ is not sufficient to conclude. It is the biinvariance of the Haar measure that allows at this point to forget about the effect of $j$ on the edges outside $\Gamma_{1, \partial}$.

Let us write this formally. Denote $\Gamma_{1}=\left\{a_{1}, \ldots, a_{k}, a_{k+1}, \ldots, a_{l}, a_{l+1}, \ldots, a_{m}\right\}$, where $\Gamma_{1, \partial}=$ $\left\{a_{1}, \ldots, a_{k}\right\}$ and $a_{k+1}, \ldots, a_{l}$ correspond to the loops along which the holonomy is imposed by 
the choice of $y_{1}$. The point for these edges is that they do not meet $\partial M_{1}$, so that their discrete holonomy is not affected by $j$. For any $i=1, \ldots, m$, denote $g_{i}^{j}=j\left(a_{i}(1)\right)^{-1} g_{i} j\left(a_{i}(0)\right)$. We have

$$
\begin{aligned}
I_{1}\left(g_{1}, \ldots, g_{k}\right)=\int_{G^{\Gamma_{1} \backslash \Gamma_{1, \partial}}} f_{1} \cdot D^{\Gamma_{1}}\left(g_{1}, \ldots, g_{m}\right) d \nu_{y_{1}}\left(g_{k+1}, \ldots, g_{l}\right) d g_{l+1} \ldots d g_{m} \\
\quad=\int_{G^{\Gamma_{1} \backslash \Gamma_{1, \partial}}} f_{1} \cdot D^{\Gamma_{1}}\left(g_{1}^{j}, \ldots, g_{m}^{j}\right) d \nu_{y_{1}} d g_{l+1} \ldots d g_{m} \\
\quad=\int_{G^{\Gamma_{1} \backslash \Gamma_{1, \partial}}} f_{1} \cdot D^{\Gamma_{1}}\left(g_{1}^{j}, \ldots, g_{k}^{j}, g_{k+1}, \ldots, g_{l}, g_{l+1}^{j}, \ldots, g_{m}^{j}\right) d \nu_{y_{1}} d g_{l+1} \ldots d g_{m} \\
\quad=\int_{G^{\Gamma_{1} \backslash \Gamma_{1, \partial}}} f_{1} \cdot D^{\Gamma_{1}}\left(g_{1}^{j}, \ldots, g_{k}^{j}, g_{k+1}, \ldots \ldots, g_{m}\right) d \nu_{y_{1}} d g_{l+1} \ldots d g_{m} \\
\quad=I_{1}\left(g_{1}^{j}, \ldots, g_{k}^{j}\right) .
\end{aligned}
$$

Now we go back to the computation (5.2). Denoting $\Gamma_{2, \partial}=\left\{g_{1}^{\prime}, \ldots, g_{k}^{\prime}\right\}$, in such a way that $g_{i}$ and $g_{i}^{\prime}$ correspond to same edge on $M$, we have

$$
\begin{aligned}
\int_{G^{\Gamma_{1} \times G^{\Gamma}}} & f_{1} f_{2} d P^{\Gamma_{1}}\left(y_{1}, x\right) d P^{\Gamma_{2}}\left(x^{-1}, y^{\prime}\right)= \\
& =\frac{1}{Z_{M_{1}}\left(y_{1}, x\right) Z_{M_{2}}\left(x^{-1}, y_{2}\right)} \int_{G^{\Gamma_{1, \partial} \times G^{\Gamma_{2}, \partial}}} I_{1}\left(g_{1}, \ldots, g_{k}\right) I_{2}\left(g_{1}^{\prime}, \ldots, g_{k}^{\prime}\right) d \nu_{x} d \nu_{x^{-1}} \\
& =\frac{1}{Z_{M_{1}}\left(y_{1}, x\right) Z_{M_{2}}\left(x^{-1}, y_{2}\right)} \int_{G^{p}} I_{1} \cdot I_{2}\left(g_{1}, \ldots, g_{k}\right) d \nu_{x}\left(g_{1}, \ldots, g_{p}\right) \\
& =\frac{1}{Z_{M_{1}}\left(y_{1}, x\right) Z_{M_{2}}\left(x^{-1}, y_{2}\right)} \int_{G^{\Gamma}} d \nu_{x} \int_{G^{\Gamma \backslash \Gamma_{\partial}}} f_{1} D^{\Gamma_{1}} f_{2} D^{\Gamma_{2}} d \nu_{y_{1}} d \nu_{y_{2}} d g^{\Gamma} \\
& =\frac{Z_{M}\left(y_{1}, x, y_{2}\right)}{Z_{M_{1}}\left(y_{1}, x\right) Z_{M_{2}}\left(x^{-1}, y_{2}\right)} \int_{G^{\Gamma}} f_{1} f_{2} d P^{\Gamma}\left(y_{1}, x, y_{2}\right) .
\end{aligned}
$$

The equality of the normalization constants follows from the case where $f_{1} f_{2}$ is identically equal to 1 , and this finishes the proof.

The relation between conditional partition functions that was just established deserves to be stated separately. We shall discuss this result and another one of the same kind in the second part of this chapter.

Proposition 5.1.3 For all $x \in G^{p}, y_{1} \in G^{p_{1}}$ and $y_{2} \in G^{p_{2}}$, the following relation holds:

$$
Z_{M}\left(y_{1}, x, y_{2}\right)=Z_{M_{1}}\left(y_{1}, x\right) Z_{M_{2}}\left(x^{-1}, y_{2}\right) .
$$

We can use the proposition 5.1.2 to prove the theorem 5.1.1.

Proof of 5.1.1. First of all, the relation (5.1) implies the conditional independence stated in the first part of the theorem. Now, by the continuity of the random holonomy, it is sufficient to prove that this relation holds for functions $f_{1}$ and $f_{2}$ that depend on the holonomies along loops that can be put into a graph. So we consider two such functions $f_{1}$ and $f_{2}$, and choose a graph $\Gamma$ on $M$ such that all the loops which we need to consider are in $\Gamma^{*}$. Choose $y_{1}$ and $y_{2}$ as in the proposition 5.1.2. Let $\Gamma_{1}, \Gamma_{2}$ and $\Gamma_{\partial}$ be the graphs on $M_{1}, M_{2}$ and $M_{1} \cap M_{2}$ induced by $\Gamma$. The functions $f_{1}$ and $f_{2}$ may be regarded as gauge-invariant functions on $G^{\Gamma_{2}}$ and $G^{\Gamma_{1}}$ 
respectively. Pick also a gauge-invariant function $f_{\partial}$ on $G^{\Gamma_{\partial}}$. This function depends only on the conjugacy classes of the holonomies along $L_{1}, \ldots, L_{p}$. So, we have:

$$
\begin{aligned}
\int_{G^{\Gamma}} & f_{1} f_{2} f_{\partial} d P\left(y, y^{\prime}\right)=\frac{1}{Z_{M}\left(y, y^{\prime}\right)} \int_{G^{\Gamma}} f_{1} f_{2} f_{\partial} D^{\Gamma} d \nu_{y} d \nu_{y^{\prime}} d g^{\prime} \\
= & \frac{1}{Z_{M}\left(y, y^{\prime}\right)} \int_{G^{p}} Z\left(y, x, y^{\prime}\right) d x \frac{1}{Z\left(y, x, y^{\prime}\right)} \int_{G^{\Gamma}} f_{1} f_{2} f_{\partial} D^{\Gamma} d \nu_{x} d \nu_{y} d \nu_{y^{\prime}} d g^{\prime} \\
= & \frac{1}{Z_{M}\left(y, y^{\prime}\right)} \int_{G^{p}} d x f_{\partial}(x) Z\left(y, x, y^{\prime}\right) \int_{G^{\Gamma}} f_{1} f_{2} d P^{\Gamma}\left(y_{1}, x, y_{2}\right) \\
= & \frac{1}{Z_{M}\left(y, y^{\prime}\right)} \int_{G^{p}} d x f_{\partial}(x) Z\left(y, x, y^{\prime}\right) \int_{G^{\Gamma_{1}}} f_{1} d P^{\Gamma_{1}}\left(y_{1}, x\right) \int_{G^{\Gamma_{2}}} f_{2} d P^{\Gamma_{2}}\left(x^{-1}, y_{2}\right),
\end{aligned}
$$

thanks to the proposition 5.1.2. This last equality, which is true for every function $f_{\partial}$ shows that

$$
\begin{aligned}
E\left[f_{1} f_{2} \mid \mathcal{A}_{\partial}\right] & =\int_{G^{\Gamma_{1}}} f_{1} d P^{\Gamma_{1}}\left(y_{1}, x\right) \int_{G^{\Gamma_{2}}} f_{2} d P^{\Gamma_{2}}\left(x^{-1}, y_{2}\right) \\
& =\mu_{M_{1}}\left(u_{1}, t\right)\left(f_{1}\right) \mu_{M_{2}}\left(t^{-1}, u_{2}\right)\left(f_{2}\right) \\
& =E\left[f_{1} \mid \mathcal{A}_{\partial}\right] E\left[f_{2} \mid \mathcal{A}_{\partial}\right]
\end{aligned}
$$

where $\mathcal{A}_{\partial}=\sigma\left(\mathcal{H}_{L_{1}}, \ldots, \mathcal{H}_{L_{p}}\right)$ and $t, u_{1}, u_{2}$ are the conjugacy classes corresponding to $x, y_{1}$ and $y_{2}$. These last equalities prove the theorem.

\subsection{Study of an example}

Consider a closed surface $M$ of genus two realized as the connected sum of two tori. Let $M_{1}$ and $M_{2}$ denote the two halves of $M$. Set $L=\partial M_{1}=-\partial M_{2}$. Let $f_{1}$ be a function on $\left(\mathcal{F}\left(L M_{1}, G\right), \mathcal{A}_{1}\right)$ and $f_{2}$ a function on $\left(\mathcal{F}\left(L M_{2}, G\right), \mathcal{A}_{2}\right)$. Then $f_{1} f_{2}$ can be seen as a function on $(\mathcal{F}(L M, G), \mathcal{A})$. We just proved that, for all $t \in G / \mathrm{Ad}, \mu_{M}(t)\left(f_{1} f_{2}\right)=\mu_{M_{1}}(t)\left(f_{1}\right) \mu_{M_{2}}\left(t^{-1}\right)\left(f_{2}\right)$. Since $\mu_{M}=\int_{G / \text { Ad }} \mu_{M}(t) Z_{M}(t) d t$ (see proposition 2.10.5), we get:

$$
\begin{aligned}
\mu_{M}\left(f_{1} f_{2}\right) & =\frac{1}{Z_{M}} \int_{G / \mathrm{Ad}} Z_{M}(t) \mu_{M}(t)\left(f_{1} f_{2}\right) d t \\
& =\frac{1}{Z_{M}} \int_{G / \mathrm{Ad}} Z_{M_{1}}(t) \mu_{M_{1}}(t)\left(f_{1}\right) Z_{M_{2}}\left(t^{-1}\right) \mu_{M_{2}}\left(t^{-1}\right)\left(f_{2}\right) d t .
\end{aligned}
$$

We rewrite this last equality in a more symmetric form:

$$
Z_{M} \mu_{M}(f)=\int_{G / \mathrm{Ad}} Z_{M_{1}}(t) \mu_{M_{1}}(t)\left(f_{1}\right) Z_{M_{2}}\left(t^{-1}\right) \mu_{M_{2}}\left(t^{-1}\right)\left(f_{2}\right) d t .
$$

The point here is that the analytic objects that glue together in a simple way are not the probability measures, but the measures with their natural weights.

Now, let us study further the relationships between the three $\sigma$-algebras $\mathcal{A}_{1}, \mathcal{A}_{2}$ and $\mathcal{A}$. For this, it is convenient to choose a base point $m$ on $L$, say $m=L(0)$ and to consider only loops based at $m$. According to the proposition 2.10.9, the random holonomies along these based loops generate the whole $\sigma$-algebra $\mathcal{A}$. Thus, we consider the probability spaces $\Omega_{M_{i}}=$ 
$\mathcal{F}\left(L_{m} M_{i}, G\right) /$ Ad and $\Omega_{M}=\mathcal{F}\left(L_{m} M, G\right) /$ Ad endowed with the $\sigma$-algebras $\mathcal{A}_{i}=\sigma\left(\mathcal{H}_{l_{1}, \ldots, l_{n}}, l \in\right.$ $\left.L_{m} M_{i}\right), i=1,2$ and $\mathcal{A}=\sigma\left(\mathcal{H}_{l_{1}, \ldots, l_{n}}, l \in L_{m} M\right)$.

The theorem 5.1.1 says that the probability spaces $\left(\Omega_{M_{i}}, \mathcal{A}_{i}, \mu_{M_{i}}\left(t^{ \pm 1}\right)\right), i=1,2$ are naturally isomorphic with two independent subspaces of $\left(\Omega_{M}, \mathcal{A}, \mu_{M}(t)\right)$. It is natural to ask whether the inclusion $\mathcal{A}_{1} \vee \mathcal{A}_{2} \subset \mathcal{A}$ is an equality or not.

In order to answer this question, choose $l_{1} \in L_{m} M_{1}$ and $l_{2} \in L_{m} M_{2}$. The random variable $\mathcal{H}_{l_{1}, L, l_{2}}$ is $\mathcal{A}$-measurable, but is it $\mathcal{A}_{1} \vee \mathcal{A}_{2}$-measurable? The random variables $\mathcal{H}_{l_{1}, L}$ and $\mathcal{H}_{L, l_{2}}$ are respectively $\mathcal{A}_{1^{-}}$and $\mathcal{A}_{2}$-measurable and it seems reasonable to believe that they provide the whole information about $\mathcal{H}_{l_{1}, L, l_{2}}$ available in $\mathcal{A}_{1} \vee \mathcal{A}_{2}$.

If $G$ is Abelian, then $\mathcal{H}_{l_{1}, L}=\left(H_{l_{1}}, H_{L}\right), \mathcal{H}_{L, l_{2}}=\left(H_{L}, H_{l_{2}}\right)$ and $\mathcal{H}_{l_{1}, L, l_{2}}=\left(H_{l_{1}}, H_{L}, H_{l_{2}}\right)$ so that $\mathcal{H}_{l_{1}, L, l_{2}}$ is certainly $\mathcal{A}_{1} \vee \mathcal{A}_{2}$-measurable. We shall prove in this case that the equality $\left(\mathcal{A}_{1} \vee \mathcal{A}_{2}\right)^{\sim}=\mathcal{A}$ holds, where the tilde denotes the completion with respect to $\mu_{M}(t)$.

But if $G$ is not Abelian, this is not true anymore. Let us consider the example $G=S O(3)$ and describe the conjugacy and joint conjugacy classes in $S O(3)$ and $S O(3)^{n}$.

If $r \in S O(3)$ is neither the identity nor a symmetry, it has an angle and an axis, which can be oriented in such a way that the angle is an element of $(0, \pi)$. Thus, $r$ has a half-axis and an angle. This angle characterizes the conjugacy class of $r$ (this is still true for the identity and the symmetries). Now, consider $\left(r_{1}, \ldots, r_{n}\right)$ and $\left(r_{1}^{\prime}, \ldots, r_{n}^{\prime}\right)$ two $n$-uples of rotations that have half-axes and angles $\left(u_{i}, \theta_{i}\right),\left(u_{j}^{\prime}, \theta_{j}^{\prime}\right)$. They belong to the same joint conjugacy class if and only if $\theta_{i}=\theta_{i}^{\prime}$ for all $i=1 \ldots n$ and if there exists a rotation $R \in S O(3)$ such that $u_{i}^{\prime}=R\left(u_{i}\right)$.

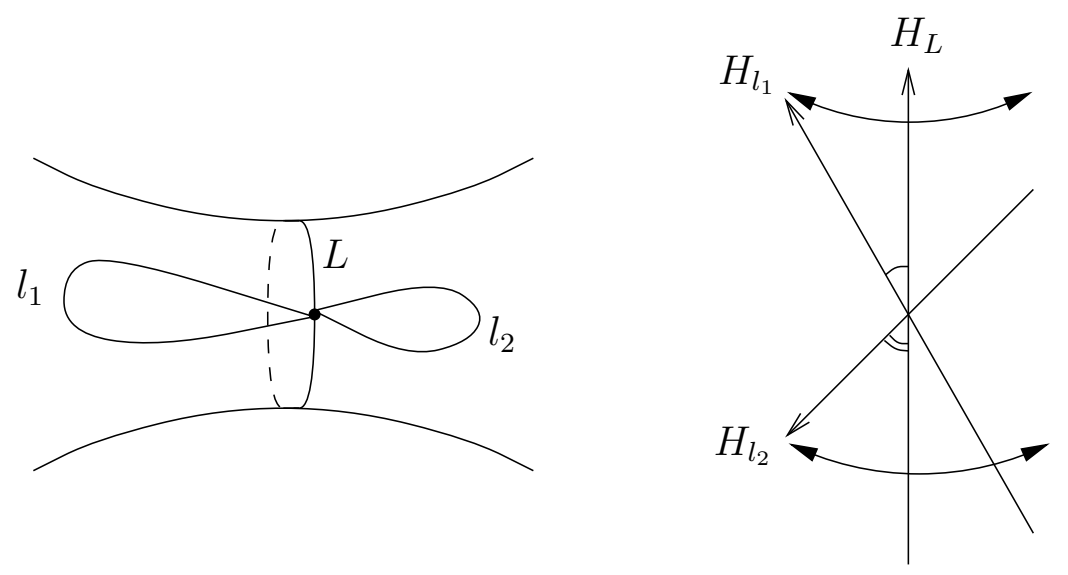

Figure 5.1: Lack of information about the joint class $\left[H_{L}, H_{l_{1}}, H_{l_{2}}\right]$ when $G=S O(3)$.

The random variables $\mathcal{H}_{l_{1}, L}$ and $\mathcal{H}_{L, l_{2}}$ determine the angles of the three rotations $H_{l_{1}}, H_{L}, H_{l_{2}}$ and the relative position of the half-axes of $H_{l_{1}}$ and $H_{L}$ on one hand and $H_{L}$ and $H_{l_{2}}$ on the other hand. But this is not enough to determine the relative position of the three half-axes (see figure 5.1). There remains an undetermined rotation around the axis of $H_{L}$. Such a rotation is an element of $S O(3)$ which commutes with $H_{L}$, in other words an element of the centralizer of $H_{L}$.

This informal argument suggests that $\mathcal{A}_{1} \vee \mathcal{A}_{2}$ is really a smaller $\sigma$-algebra than $\mathcal{A}$. On the other hand, if the distributions of $H_{l_{1}}$ and $H_{l_{2}}$ are not singular with respect to the Haar measure, which is the case if $l_{1}$ and $l_{2}$ are not constant loops, it is possible to extract from the random variable $\mathcal{H}_{l_{1}, L, l_{2}}$ the amount of information that it is necessary to add to $\mathcal{A}_{1} \vee \mathcal{A}_{2}$ to recover $\mathcal{A}$. 
Recall that $t$ is an element of $S O(3) / \mathrm{Ad}$ and that $\mathcal{H}_{L}=t \mu_{M}(t)$-almost surely. Pick an element $x \in t$. Suppose that we are given a measurable section $\tau:(t \times S O(3)) / \mathrm{Ad} \longrightarrow$ $\{x\} \times S O(3)$ of the canonical projection. A realization of $\mathcal{H}_{l_{1}, L, l_{2}}=\left[H_{l_{1}}, H_{L}, H_{l_{2}}\right]$ determines $\mu_{M}(t)$-a.s. two classes of $(t \times S O(3)) / \mathrm{Ad}$, namely $\left[H_{L}, H_{l_{1}}\right]$ and $\left[H_{L}, H_{l_{2}}\right]$. Let $\left(x, x_{1}\right)$ and $\left(x, x_{2}\right)$ be the images by $\tau$ of these classes. It is easy to check that there exists an element $z$ of the centralizer $C(x)$ of $x$ such that $\mathcal{H}_{l_{1}, L, l_{2}}=\left[x_{1}, x, z^{-1} x_{2} z\right]$ and that this $z$ is determined up to right and left multiplication by an element of $C\left(x_{1}\right) \cap C\left(x_{2}\right)$, which is almost surely equal to the center $Z(G)$ of $G$, so that the class $z Z(G) \in C(x) / Z(G)$ is well defined.

Thus, we outlined the construction of a random variable $Z$ with values in $C(x) / Z(G)$, which can be seen as the missing angle of the figure 5.1. We will show that it has a uniform distribution, that it is independent of $\mathcal{A}_{1} \vee \mathcal{A}_{2}$ and that $\left(\mathcal{A}_{1} \vee \mathcal{A}_{2} \vee \sigma(Z)\right)^{\sim}=\mathcal{A}^{\sim}$. Note that if $G$ is Abelian, the space $C(x) / Z(G)$ is just a point, so this is not in contradiction with the fact that $\left(\mathcal{A}_{1} \vee \mathcal{A}_{2}\right)^{\sim}=\mathcal{A}^{\sim}$.

\subsection{Sewing of two surfaces along one circle}

We consider the general situation described at the beginning of the chapter, with $p=1$, i.e. when the two surfaces are glued together along only one circle $L$. Pick $x \in t$ and choose elements $u_{1}$ and $u_{2}$ in $(G \mathrm{Ad})^{p_{1}}$ and $(G / \mathrm{Ad})^{p_{2}}$, representing the holonomies along the other components of the boundaries of $M_{1}$ and $M_{2}$ and maybe other loops inside $M_{1}$ and $M_{2}$.

Theorem 5.3.1 1. If $G$ is Abelian, then the completions of the $\sigma$-algebras $\mathcal{A}_{1} \vee \mathcal{A}_{2}$ and $\mathcal{A}$ with respect to the measure $\mu_{M}\left(u_{1}, t, u_{2}\right)$ are equal.

2. If $G$ is not Abelian, there exists a non-trivial sub- $\sigma$-algebra $\mathcal{Z}$ of $\mathcal{A}$ which is independent of $\mathcal{A}_{1} \vee \mathcal{A}_{2}$ and such that the completions of $\mathcal{A}_{1} \vee \mathcal{A}_{2} \vee \mathcal{Z}$ and $\mathcal{A}$ with respect to $\mu_{M}\left(u_{1}, t, u_{2}\right)$ are equal.

By a non-trivial $\sigma$-algebra, we mean a $\sigma$-algebra which is not independent of itself, or equivalently, that contains sets of probability different from 0 and 1.

The proof of this theorem requires a certain amount of preliminary work, that leads to some interesting results. Let us choose on $M$ a Riemannian metric such that $\partial M$ and $L$ are geodesic. Recall that $m=L(0)$. The set of piecewise geodesic loops on $M$ based at $m$ is denoted by $P G L_{m} M$.

Lemma 5.3.2 On the space $\Omega_{M}$, the $\sigma$-algebra $\mathcal{A}$ is contained in $\mathcal{A}_{12}$, which is the completion of $\sigma\left(\mathcal{H}_{\lambda_{1}, \ldots, \lambda_{n}}, \lambda_{1}, \ldots, \lambda_{n} \in P G L_{m} M_{1} \cup P G L_{m} M_{2}\right)$ with respect to $\mu_{M}\left(u_{1}, t, u_{2}\right)$. In particular, $\mathcal{A}^{\sim}=\mathcal{A}_{12}$.

Proof. By the multiplicativity of the random holonomy described at the end of section 2.10.3, the holonomies along loops that are finite products of loops of $L_{m} M_{1}$ and $L_{m} M_{2}$ are $\mathcal{A}_{12}$-measurable. According to the continuity property stated in 2.10 .7 and to the fact that the holonomy depends only on the equivalence class of the loops, the result depends on the fact that any loop of $L_{m} M$ can be approximated by loops that are equivalent to finite products of loops of $P G L_{m} M_{1}$ and $P G L_{m} M_{2}$. Consider a piecewise geodesic loop of $L_{m} M$. This loop cuts $L$ transversally at most a finite number of times, hence it is equivalent to a finite product of loops of $P G L_{m} M_{1}$ and $P G L_{m} M_{2}$. Since any loop of $L_{m} M$ can be approximated by piecewise 
geodesic loops, the result is proved.

According to this lemma, we restrict our attention to the loops based at $m$ and whose image is either in $M_{1}$ or in $M_{2}$. This is enough to prove the first part of the theorem: if $G$ is Abelian, a random variable of the form $\mathcal{H}_{\lambda_{1}, \ldots, \lambda_{n}}$ with $\lambda_{1}, \ldots, \lambda_{n} \in P G L_{m} M_{1} \cup P G L_{m} M_{2}$ is just $\left(H_{\lambda_{1}}, \ldots, H_{\lambda_{n}}\right)$ and it is certainly measurable with respect to $\mathcal{A}_{1} \vee \mathcal{A}_{2}$, since each variable $H_{\lambda_{i}}$ is either $\mathcal{A}_{1}$ - or $\mathcal{A}_{2}$-measurable.

If $G$ is not Abelian, we need to compute the conditional distribution of a random variable like $\mathcal{H}_{\lambda_{1}, \ldots, \lambda_{n}}$ with respect to $\mathcal{A}_{1} \vee \mathcal{A}_{2}$. For this, it is convenient to introduce the following notation.

Recall that $x$ is a fixed element in $t \in G / \mathrm{Ad}$. Consider an element of $\left(t \times G^{n}\right) / \mathrm{Ad}$. It is a class that can be written under the form $\left[x, x_{1}, \ldots, x_{n}\right]$, where the $n$-uple $\left(x_{1}, \ldots, x_{n}\right)$ is defined up to conjugation by an element of the centralizer $C(x)$. The measure

$$
\int_{C(x)} \delta_{\left(z^{-1} x_{1} z, \ldots, z^{-1} x_{n} z\right)} d z
$$

is well defined on $G^{n}$, depending only on the class $\left[x, x_{1}, \ldots, x_{n}\right]$ and on the choice of $x$. We denote it by $\pi_{x}^{n}\left(\left[x, x_{1}, \ldots, x_{n}\right]\right)$. Note that if $x^{\prime}=y^{-1} x y$, then for any function $f$ on $G^{n}$,

$$
\int_{G^{n}} f d \pi_{x^{\prime}}^{n}\left(\left[x, x_{1}, \ldots, x_{n}\right]\right)=\int_{G^{n}} f \circ \operatorname{Ad}(y) d \pi_{x}^{n}\left(\left[x, x_{1}, \ldots, x_{n}\right]\right) .
$$

Proposition 5.3.3 Let $\lambda_{1}, \ldots, \lambda_{n}$ be $n$ loops of $P G L_{m} M_{1}$ and $\lambda_{1}^{\prime}, \ldots, \lambda_{n^{\prime}}^{\prime}$ be $n^{\prime}$ loops of $P G L_{m} M_{2}$. For any continuous function $f$ on $G^{n+n^{\prime}}$ invariant by diagonal adjunction, we have

$$
\begin{aligned}
& E_{\mu_{M}\left(u_{1}, t, u_{2}\right)}\left[f\left(\mathcal{H}_{\lambda_{1}, \ldots, \lambda_{n}, \lambda_{1}^{\prime}, \ldots, \lambda_{n^{\prime}}^{\prime}}\right) \mid \mathcal{A}_{1} \vee \mathcal{A}_{2}\right]= \\
& \qquad \int_{G^{n+n^{\prime}}} f d \pi_{x}^{n}\left(\mathcal{H}_{L, \lambda_{1}, \ldots, \lambda_{n}}\right) \otimes d \pi_{x}^{n^{\prime}}\left(\mathcal{H}_{L, \lambda_{1}^{\prime}, \ldots, \lambda_{n^{\prime}}^{\prime}}\right) \text { a.s. }
\end{aligned}
$$

The right hand side does not depend on $x$, only on $t$, because of the invariance of $f$ and the relation (5.3).

Proof. Choose $l_{1}, \ldots, l_{m} \in P G L_{m} M_{1}$ and $l_{1}^{\prime}, \ldots, l_{m^{\prime}}^{\prime} \in P G L_{m} M_{2}$. Let $f_{1}$ be a continuous function on $G^{m} / \operatorname{Ad} \times G^{m^{\prime}} / \mathrm{Ad}$. Let $\Gamma$ be a graph on $M$ such that $L$, the $l_{i}$ 's, the $l_{i}^{\prime}$ 's, the $\lambda_{i}$ 's and the $\lambda_{i}^{\prime}$ 's are in $\Gamma^{*}$. As in the proof of the theorem 5.1.1, denote by $\Gamma_{1}$ and $\Gamma_{2}$ the graphs induced by $\Gamma$ on $M_{1}$ and $M_{2}$. Let $f$ be a continuous function on $G^{n+n^{\prime}}$ invariant by diagonal adjunction. Using the proposition 5.1.2, we get

$$
\begin{gathered}
E_{\mu_{M}\left(u_{1}, t, u_{2}\right)}\left[f\left(\mathcal{H}_{\lambda_{1}, \ldots, \lambda_{n}, \lambda_{1}^{\prime}, \ldots, \lambda_{n^{\prime}}^{\prime}}\right) f_{1}\left(\mathcal{H}_{l_{1}, \ldots, l_{m}}, \mathcal{H}_{l_{1}^{\prime}, \ldots, l_{m^{\prime}}^{\prime}}\right)\right]= \\
=\int_{G^{\Gamma}} f\left(h_{\lambda_{1}}, \ldots, h_{\lambda_{n}}, h_{\lambda_{1}^{\prime}}, \ldots, h_{\lambda_{n^{\prime}}^{\prime}}\right) f_{1}\left(\left[h_{l_{1}}, \ldots, h_{l_{m}}\right],\left[h_{l_{1}^{\prime}}, \ldots, h_{l_{m^{\prime}}^{\prime}}\right]\right) d P^{\Gamma}\left(y, x, y^{\prime}\right) \\
=\int_{G^{\Gamma_{1} \times G^{\Gamma}}} f\left(h_{\lambda_{1}}, \ldots, h_{\lambda_{n}}, h_{\lambda_{1}^{\prime}}, \ldots, h_{\lambda_{n^{\prime}}}\right) f_{1}\left(\left[h_{l_{1}}, \ldots, h_{l_{m}}\right],\left[h_{l_{1}^{\prime}}, \ldots, h_{l_{m^{\prime}}^{\prime}}\right]\right) \\
d P^{\Gamma_{1}}(y, x) d P^{\Gamma_{2}}\left(x^{-1}, y^{\prime}\right) .
\end{gathered}
$$

For each element $z \in C(x)$ and each $i=1,2$, the gauge transformation equal to 1 at each vertex of $\Gamma_{i}$ except at $m$ where it takes the value $z$ leaves $P^{\Gamma_{i}}$ invariant. Thus, the last integral is equal 
to

$$
\begin{aligned}
& \int_{G^{\Gamma_{1} \times G^{\Gamma_{2}}}} \int_{C(x)^{2}} f\left(z_{1}^{-1} h_{\lambda_{1}} z_{1}, \ldots, z_{1}^{-1} h_{\lambda_{n}} z_{1}, z_{2}^{-1} h_{\lambda_{1}^{\prime}} z_{2}, \ldots, z_{2}^{-1} h_{\lambda_{n^{\prime}}^{\prime}} z_{2}\right) d z_{1} d z_{2} \\
& f_{1}\left(\left[h_{l_{1}}, \ldots, h_{l_{m}}\right],\left[h_{l_{1}^{\prime}}, \ldots, h_{l_{m^{\prime}}^{\prime}}\right]\right) d P^{\Gamma_{1}}(y, x) d P^{\Gamma_{2}}\left(x^{-1}, y^{\prime}\right) \\
& =\int_{G^{\Gamma_{1}} \times G^{\Gamma_{2}}} \int_{G^{n+n^{\prime}}} f d \pi_{x}^{n}\left(\left[h_{L}, h_{\lambda_{1}}, \ldots, h_{\lambda_{n}}\right]\right) \otimes d \pi_{x}^{n^{\prime}}\left(\left[h_{L}, h_{\lambda_{1}^{\prime}}, \ldots, h_{\lambda_{n^{\prime}}^{\prime}}\right]\right) \\
& f_{1}\left(\left[h_{l_{1}}, \ldots, h_{l_{m}}\right],\left[h_{l_{1}^{\prime}}, \ldots, h_{l_{m^{\prime}}^{\prime}}\right]\right) d P^{\Gamma_{1}}(y, x) d P^{\Gamma_{2}}\left(x^{-1}, y^{\prime}\right) \\
& =E\left[\int_{G^{n+n^{\prime}}} f d \pi_{x}^{n}\left(\left[\mathcal{H}_{L, \lambda_{1}, \ldots, \lambda_{n}}\right]\right) \otimes d \pi_{x}^{n^{\prime}}\left(\left[\mathcal{H}_{L, \lambda_{1}^{\prime}, \ldots, \lambda_{n^{\prime}}^{\prime}}\right]\right) f_{1}\left(\mathcal{H}_{l_{1}, \ldots, l_{m}}, \mathcal{H}_{l_{1}^{\prime}, \ldots, l_{m^{\prime}}}\right)\right] \text {, }
\end{aligned}
$$

which proves the proposition.

Remark that the "reasonable" intuition that the variables $\mathcal{H}_{L, \lambda_{1}, \ldots, \lambda_{n}}$ and $\mathcal{H}_{L, \lambda_{1}^{\prime}, \ldots, \lambda_{n^{\prime}}^{\prime}}$ contain all the information about $\mathcal{H}_{\lambda_{1}, \ldots, \lambda_{n}, \lambda_{1}^{\prime}, \ldots, \lambda_{n^{\prime}}^{\prime}}$ available in $\mathcal{A}_{1} \vee \mathcal{A}_{2}$ is confirmed.

The next step is the construction of the variable $Z$ in a general context. We use the same method as in the example treated informally in section 5.2 but this requires some technical results.

Lemma 5.3.4 Let $G$ be a compact connected Lie group of dimension $n$ and rank $k$. Set $N=$ $n-k+1$. The set $S_{N}$ of all $N$-uples $\left(g_{1}, \ldots, g_{N}\right)$ such that the closed subgroup of $G$ generated by $g_{1}, \ldots, g_{N}$ is $G$ itself has full Haar measure in $G^{N}$. Moreover, $S_{N}$ is stable by diagonal adjunction, i.e. $\left(g_{1}, \ldots, g_{N}\right)$ belongs to $S_{N}$ if and only if $\left(h^{-1} g_{1}, h, \ldots, h^{-1} g_{N} h\right)$ does for any $h$ in $G$.

Proof. The key of this result is that almost every element of $G$ is regular, i.e. generates a maximal torus [Si]. Let $g_{1}$ be such a regular element. It generates a subgroup $G_{1}=T_{1}$ of $G$ of dimension $k$. If $G_{1}$ is a proper subgroup of $G$, then $\operatorname{dim} G_{1}<\operatorname{dim} G$ since $G$ is connected. Thus, the complementary of $G_{1}$ has full measure as well as the set of regular elements outside $G_{1}$. Let $g_{2}$ be such an element and $T_{2}$ the torus that it generates. Denote by $G_{2}$ the subgroup generated by $\left\{g_{1}, g_{2}\right\}$. Denote by $\mathfrak{g}_{1}, \mathfrak{g}_{2}$ and $\mathfrak{t}_{2}$ the Lie algebras of $G_{1}, G_{2}$ and $T_{2}$ respectively. The fact that $\mathfrak{t}_{2} \not \subset \mathfrak{g}_{1}$ and $\mathfrak{g}_{2} \supset \mathfrak{g}_{1}+\mathfrak{t}_{2}$ shows that $\operatorname{dim} G_{2}>\operatorname{dim} G_{1}$. Repeating this procedure $N=n-k+1$ times, we get a subgroup $G_{N}$ which is equal to $G$. It is clear from this construction that the set $S_{N}$ of convenient $N$-uples has full Haar measure in $G^{N}$.

The last statement depends on the fact that two conjugate $N$-uples generate two conjugate subgroups of $G$.

In particular, note that if $\left(g_{1}, \ldots, g_{N}\right) \in S_{N}$, then $C\left(g_{1}\right) \cap \ldots \cap C\left(g_{N}\right)=Z(G)$.

Before to prove the next lemma, let us recall that, by a classical result of Kuratowski [Ku], a one-to-one measurable map between two Polish spaces sends Borel subsets to Borel subsets.

Lemma 5.3.5 There exists a measurable section $\tau:\left(t \times G^{N}\right) / \mathrm{Ad} \longrightarrow\{x\} \times G^{N}$.

Proof. We use a theorem of Bourbaki (Topologie, chap. IX, $\S 6, \mathrm{~N}^{o} 9$, th. 5) [B1], which says that there exists a Borel subset $R$ of $t \times G^{N}$ that meets once and only once each orbit of the action of $G$. This subset $R$ allows to define a section $\tau$. To prove that $\tau$ is measurable, consider 
a Borel subset $B \subset t \times G^{N}$. The fact that $\tau^{-1}(B)=p(B \cap R)$, where $p: t \times G^{N} \longrightarrow\left(t \times G^{N}\right) / G$ is the natural projection, together with the result of Kuratowski mentionned above, shows that $\tau^{-1}(B)$ is a Borel subset. Thus, $\tau$ is measurable.

Let us choose now $2 N$ loops $, L_{1}^{1}, \ldots, L_{N}^{1} \in P G L_{m} M_{1}$ and $L_{1}^{2}, \ldots, L_{N}^{2} \in P G L_{m} M_{2}$, such that the distribution of $\left(H_{L_{1}^{1}}, \ldots, H_{L_{N}^{1}}, H_{L_{1}^{2}}, \ldots, H_{L_{N}^{2}}\right)$ has a density with respect to the Haar measure on $G^{2 N}$. This is a weak condition on the $L_{j}^{i}$ 's which ensures that the set where $\left(H_{L_{1}^{i}}, \ldots, H_{L_{N}^{i}}\right)$ belongs to $S_{N}, i=1,2$ has full Yang-Mills measure. Let us fix $\omega$ inside this set. Set $\tau\left(\mathcal{H}_{L, L_{1}^{1}, \ldots, L_{N}^{1}}(\omega)\right)=\left(x, x_{1}^{1}, \ldots, x_{N}^{1}\right)$ and $\tau\left(\mathcal{H}_{L, L_{1}^{2}, \ldots, L_{N}^{2}}(\omega)\right)=\left(x, x_{1}^{2}, \ldots, x_{N}^{2}\right)$, where $\tau$ is a measurable section given by the preceding lemma. Choose an element $\left(y_{1}^{1}, \ldots, y_{N}^{1}, x, y_{1}^{2}, \ldots, y_{N}^{2}\right)$ inside the class $\mathcal{H}_{L_{1}^{1}, \ldots, L_{N}^{1}, L, L_{1}^{2}, \ldots, L_{N}^{2}}(\omega)$. For $i=1,2$, the equality $\left[x, x_{1}^{i}, \ldots, x_{N}^{i}\right]=\left[x, y_{1}^{i}, \ldots, y_{N}^{i}\right]$ implies the existence of $z_{i} \in C(x)$ such that $y_{1}^{i}=z_{i}^{-1} x_{1}^{i} z_{i}, \ldots, y_{N}^{i}=z_{i}^{-1} x_{N}^{i} z_{i}$. Thus there exists $z \in C(x)$ such that

$$
\left[y_{1}^{1}, \ldots, y_{N}^{1}, x, y_{1}^{2}, \ldots, y_{N}^{2}\right]=\left[x_{1}^{1}, \ldots, x_{N}^{1}, x, z^{-1} x_{1}^{2} z, \ldots, z^{-1} x_{N}^{2} z\right],
$$

for example $z=z_{2} z_{1}^{-1}$. If another element $z^{\prime} \in C(x)$ satisfies the same relation, then it is easily checked that there exist two elements $z^{\prime \prime} \in C\left(x_{1}^{1}\right) \cap \ldots \cap C\left(x_{N}^{1}\right)=Z(G)$ and $z^{\prime \prime \prime} \in$ $C\left(x_{1}^{2}\right) \cap \ldots \cap C\left(x_{N}^{2}\right)=Z(G)$ such that $z^{\prime}=z^{\prime \prime} z z^{\prime \prime \prime}=z z^{\prime \prime} z^{\prime \prime \prime}$. Thus, the class $z Z(G) \in C(x) / Z(G)$ is well defined and we define the value at $\omega$ of the random variable $Z$ by

$$
Z(\omega)=z Z(G)
$$

Note that $Z$ depends on $\omega$ only through $\mathcal{H}_{L_{1}^{1}, \ldots, L_{N}^{1}, L, L_{1}^{2}, \ldots, L_{N}^{2}}(\omega)$ so that we may write $Z=$ $\widetilde{Z}\left(\mathcal{H}_{L_{1}^{1}, \ldots \ldots, L_{N}^{2}}\right)$ for some function $\widetilde{Z}: G^{2 N+1} / \operatorname{Ad} \longrightarrow C(x) / Z(G)$. Moreover, for any $z_{1}, z_{2}$ in $C(x)$ and any $x^{1}, x^{2}$ in $G^{N}$, it is easily checked that

$$
\widetilde{Z}\left(\left[z_{1}^{-1} x^{1} z_{1}, x, z_{2}^{-1} x^{2} z_{2}\right]\right)=z_{1} z_{2}^{-1} \widetilde{Z}\left(\left[x^{1}, x, x^{2}\right]\right) .
$$

Proposition 5.3.6 The random variable $Z$ is uniformly distributed on the Lie group $C(x) / Z(G)$ and independent of $\mathcal{A}_{1} \vee \mathcal{A}_{2}$ under the measure $\mu_{M}\left(u_{1}, t, u_{2}\right)$.

Since $Z(G)$ is a closed normal subgroup of $C(x)$, the quotient $C(x) / Z(G)$ is still a Lie group, so that it makes sense to speak about uniform distribution.

Proof. Let $f$ be a continuous function on $C(x) / Z(G)$. It is more convenient for the notations to consider $\widetilde{Z}$ as a function of the variable $\mathcal{H}_{L_{1}^{1}, \ldots, L_{N}^{1}, L, L, L_{1}^{2}, \ldots, L_{N}^{2}}$, with twice the loop $L$. Using the proposition 5.3.3, we find

$$
\begin{aligned}
E\left[f(Z) \mid \mathcal{A}_{1} \vee \mathcal{A}_{2}\right] & =E_{\mu_{M}\left(u_{1}, t, u_{2}\right)}\left[f\left(\widetilde{Z}\left(\mathcal{H}_{L_{1}^{1}, \ldots, L_{N}^{1}, L, L, L_{1}^{2}, \ldots, L_{N}^{2}}\right)\right) \mid \mathcal{A}_{1} \vee \mathcal{A}_{2}\right] \\
& =\int_{G^{2 n+2}} f \circ \widetilde{Z} d \pi_{x}\left(\mathcal{H}_{L, L_{1}^{1}, \ldots, L_{N}^{1}}\right) \otimes \delta_{(x, x)} \otimes \pi_{x}\left(\mathcal{H}_{L, L_{1}^{2}, \ldots, L_{N}^{2}}\right) .
\end{aligned}
$$

Let us fix $\omega$ and set $\tau\left(\mathcal{H}_{L, L_{1}^{1}, \ldots, L_{N}^{1}}(\omega)\right)=\left(x, x^{1}\right), \tau\left(\mathcal{H}_{L, L_{1}^{2}, \ldots, L_{N}^{2}}(\omega)\right)=\left(x, x^{2}\right)$, where $x^{1}$ and $x^{2}$ are elements of $G^{N}$. Then

$$
E\left[f(Z) \mid \mathcal{A}_{1} \vee \mathcal{A}_{2}\right](\omega)=\int_{C(x)^{2}} f\left(\widetilde{Z}\left(z_{1}^{-1} x^{1} z_{1}, x, x, z_{2}^{-1} x^{2} z_{2}\right)\right) d z_{1} d z_{2}
$$




$$
\begin{aligned}
& =\int_{C(x)^{2}} f\left(z_{1} z_{2}^{-1} \widetilde{Z}\left(\mathcal{H}_{L_{1}^{1}, \ldots, L_{N}^{1}, L, L, L_{1}^{2}, \ldots, L_{N}^{2}}\right)\right) d z_{1} d z_{2} \\
& =\int_{C(x)} f(z) d z .
\end{aligned}
$$

Since this conditional expectation is a constant for any $f, Z$ is independent of $\mathcal{A}_{1} \vee \mathcal{A}_{2}$.

The proof of the theorem is almost finished, there only remains to prove that $\mathcal{A}_{1} \vee \mathcal{A}_{2} \vee \sigma(Z)$ contains $\mathcal{A}_{12}$.

Lemma 5.3.7 The random variable $\mathcal{H}_{L_{1}^{1}, \ldots, L_{N}^{1}, L, L_{1}^{2}, \ldots, L_{N}^{2}}$ is measurable with respect to the $\sigma$ algebra $\mathcal{A}_{1} \vee \mathcal{A}_{2} \vee \sigma(Z)$.

Proof. Let us denote by $\tau^{\prime}:\left(t \times G^{N}\right) / \mathrm{Ad} \longrightarrow G^{N}$ the composition of $\tau$ with the projection on the $N$ last factors. Then, by construction of $Z$, the following equality holds almost surely:

$$
\mathcal{H}_{L_{1}^{1}, \ldots, L_{N}^{1}, L, L_{1}^{2}, \ldots, L_{N}^{2}}=\left[\tau^{\prime}\left(\mathcal{H}_{L, L_{1}^{1}, \ldots, L_{N}^{1}}\right), x, Z^{-1} \tau^{\prime}\left(\mathcal{H}_{L, L_{1}^{2}, \ldots, L_{N}^{2}}\right) Z\right]
$$

proving the lemma.

The next proposition will finish the proof:

Proposition 5.3.8 The $\sigma$-algebra $\mathcal{A}_{1} \vee \mathcal{A}_{2} \vee \sigma\left(\mathcal{H}_{L_{1}^{1}, \ldots, L_{N}^{1}, L, L_{1}^{2}, \ldots, L_{N}^{2}}\right)$ contains $\mathcal{A}_{12}$.

Proof. Pick $p$ loops $l_{1}, \ldots, l_{p}$ in $L_{m} M_{1}$ and $q$ loops $l_{1}^{\prime}, \ldots, l_{q}^{\prime}$ in $L_{m} M_{2}$. We abbreviate by $l, l^{\prime}, L^{1}$ and $L^{2}$ the corresponding families of loops.

We will show that the random variables $\mathcal{H}_{l, L^{1}}, \mathcal{H}_{L^{2}, l^{\prime}}$ and $\mathcal{H}_{L^{1}, L^{2}}$ determine $\mathcal{H}_{l, l^{\prime}}$ if the values of $\mathcal{H}_{L^{1}}$ and $\mathcal{H}_{L^{2}}$ are in $S_{N} / \mathrm{Ad}$. We do not even restrict ourselves to piecewise geodesic loops. Since $\mathcal{H}_{l, l^{\prime}}$ is just a continuous projection of $\mathcal{H}_{l, L^{1}, L^{2}, l^{\prime}}$, it is sufficient to write this last variable as a function of the three given variables. For this, we construct a map:

$$
\left(G^{p} \times S_{N}\right) / \operatorname{Ad} \times_{S_{N}}\left(S_{N} \times S_{N}\right) / \operatorname{Ad} \times_{S_{N}}\left(S_{N} \times G^{q}\right) / \operatorname{Ad} \stackrel{\kappa}{\longrightarrow}\left(G^{p} \times S_{N} \times S_{N} \times G^{q}\right) / \operatorname{Ad} .
$$

The symbols $\times_{S_{N}}$ above mean that the map $\kappa$ is only defined on the set of elements of the form $\left(\left[u_{1}, \ldots, u_{p}, g_{1}, \ldots, g_{N}\right],\left[g_{1}^{\prime}, \ldots, g_{N}^{\prime}, h_{1}^{\prime}, \ldots, h_{N}^{\prime}\right],\left[h_{1}, \ldots, h_{N}, v_{1}, \ldots, v_{q}\right]\right)$ such that $\left[g_{1}, \ldots, g_{N}\right]=$ $\left[g_{1}^{\prime}, \ldots, g_{N}^{\prime}\right]$ and $\left[h_{1}, \ldots, h_{N}\right]=\left[h_{1}^{\prime}, \ldots, h_{N}^{\prime}\right]$.

We claim that it makes sense to construct $\kappa$ such that the image of such a triple is the unique element $\left[x_{1}, \ldots, x_{p}, r_{1}, \ldots, r_{N}, s_{1}, \ldots, s_{N}, y_{1}, \ldots, y_{q}\right]$ such that, with compact notations, $[x, r]=[u, g],[r, s]=\left[g^{\prime}, h^{\prime}\right]$ and $[s, y]=[h, v]$. It is not difficult to see that such an element exists: if $z_{g}$ and $z_{h}$ are two elements of $G$ such that $\operatorname{Ad}\left(z_{g}\right) g=g^{\prime}$ and $\operatorname{Ad}\left(z_{h}\right) h=h^{\prime}$, then $\left[\operatorname{Ad}\left(z_{g}\right) u, g^{\prime}, h^{\prime}, \operatorname{Ad}\left(z_{h}\right) v\right]$ is a possible choice.

Suppose that $[x, r, s, y]$ and $\left[x^{\prime}, r^{\prime}, s^{\prime}, y^{\prime}\right]$ are two candidates. Since $[x, r]=\left[x^{\prime}, r^{\prime}\right]$, there exists $z_{r} \in G$ such that $\operatorname{Ad}\left(z_{r}\right) x=x^{\prime}$ and $\operatorname{Ad}\left(z_{r}\right) r=r^{\prime}$. Similarly, there exists $z_{s}$ such that $\operatorname{Ad}\left(z_{s}\right) y=$ $y^{\prime}$ and $\operatorname{Ad}\left(z_{s}\right) s=s^{\prime}$. Now, $[r, s]=\left[r^{\prime}, s^{\prime}\right]$ implies $[r, s]=\left[\operatorname{Ad}\left(z_{r}\right) r, \operatorname{Ad}\left(z_{s}\right) s\right]=\left[\operatorname{Ad}\left(z_{r} z_{s}^{-1}\right) r, s\right]$. This forces $z_{r} z_{s}^{-1}$ to be an element of $Z(G)$, so that $\operatorname{Ad}\left(z_{r}\right)=\operatorname{Ad}\left(z_{s}\right)$ and the two candidates are equal.

We have $\mu_{M}(t)$-almost surely $\mathcal{H}_{l, L^{1}, L^{2}, l^{\prime}}=\kappa\left(\mathcal{H}_{l, L^{1}}, \mathcal{H}_{L^{1}, L^{2}}, \mathcal{H}_{L^{2}, l^{\prime}}\right)$, so that it remains only to prove that $\kappa$ is measurable. To see this, remark that $\kappa^{-1}$ is easier to define than $\kappa$ : it is a 
restriction of three continuous projections defined on $\left(G^{p} \times G^{N} \times G^{N} \times G^{q}\right) /$ Ad. Moreover, since $\kappa$ is well defined, $\kappa^{-1}$ is injective. Thus, by the result of Kuratowski mentionned above, $\kappa^{-1}$ sends Borel subsets to Borel subsets, which means exactly that $\kappa$ is measurable.

\subsection{Cutting and gluing handles}

An analysis very similar to that of the three preceding sections can be done in the case of a surface $M$ obtained by sewing together two components of the boundary of a surface $M_{1}$. There is no more conditional independence in this situation, but it is still possible to study the relationships between the measures $\mu_{M_{1}}$ and $\mu_{M}$.

Let $M_{1}$ be a surface with boundary such that $\partial M_{1}$ has at least two components $N_{1}$ and $N_{2}$. Let $\psi: N_{1} \longrightarrow N_{2}$ be an orientation-reversing diffeomorphism and let $M$ be obtained by gluing $M_{1}$ along $\psi$. Let $L_{1}$ and $L_{2}$ be two loops on $M_{1}$ whose images are $N_{1}$ and $-N_{2}$ respectively, and call $L$ the corresponding loop on $M$. Set $m=L(0), m_{1}=L_{1}(0)$ and $m_{2}=L_{2}(0)$. Note that, in contrast to the preceding situation, $M_{1}$ is not embedded in $M$, it is only immersed. Nevertheless, this immersion allows us to map $L M_{1}$ into $L M$. So, a function $f$ on $\mathcal{F}\left(L M_{1}, G\right)$ can be seen as a function $\tilde{f}$ on $\mathcal{F}(L M, G)$ and $f$ is measurable with respect to $\mathcal{A}_{1}$ if and only if $\widetilde{f}$ is measurable with respect to $\widetilde{\mathcal{A}}_{1}=\sigma\left(\mathcal{H}_{l_{1}, \ldots, l_{n}}, l \in L M_{1}\right)$. As before, we identify $f$ and $\mathcal{A}_{1}$ with $\widetilde{f}$ and $\widetilde{\mathcal{A}_{1}}$.

Theorem 5.4.1 Let $f$ be a measurable function on $\left(\mathcal{F}\left(L M_{1}, G\right), \mathcal{A}_{1}\right)$. Then for any $u_{1}, \ldots, u_{p}, t \in$ G/ Ad, the following equality holds:

$$
\mu_{M}\left(u_{1}, \ldots, u_{p}, t\right)(f)=\mu_{M_{1}}\left(u_{1}, \ldots, u_{p}, t, t^{-1}\right)(f),
$$

where the $u_{p}$ are elements of $G /$ Ad that stand for the value of the holonomy along other boundary components of $\partial M_{1}$ and maybe some other loops inside $M_{1}$.

As for the proof of the theorem 5.1.1, we begin with a discrete result. Let $i$ denote the immersion of $M_{1}$ into $M$. Given a graph $\Gamma$ on $M$, there exists a graph $\Gamma_{1}$ on $M_{1}$ such that $\Gamma_{1}$ is mapped by $i$ to $\Gamma$ and such that the edges of $\Gamma_{1}$ lying on $N_{1}$ and $N_{2}$ respectively are in one-to-one correspondence via the diffeomorphism $\psi$. We call $\Gamma_{1}$ the graph on $M_{1}$ induced by $\Gamma$. Recall that $m_{1}=L_{1}(0)$ and $m_{2}=L_{2}(0)$.

Proposition 5.4.2 Let $\Gamma$ be a graph on $M$ and $\Gamma_{1}$ the graph induced on $M_{1}$ by $\Gamma$. Let $f$ be a continuous function on $G^{\Gamma_{1}}$ which is invariant by the gauge transformations $j_{1}$ such that $j_{1}\left(m_{1}\right)=j_{1}\left(m_{2}\right)=1$. Then $f$ gives rise to a function on $G^{\Gamma}$ invariant by the gauge transformations $j$ such that $j(m)=1$ and for any $x \in G$,

$$
\int_{G^{\Gamma_{1}}} f d P^{\Gamma_{1}}\left(y, x, x^{-1}\right)=\int_{G^{\Gamma}} f d P^{\Gamma}(y, x),
$$

where $x$ is the holonomy along $N_{1}$ and $-N_{2}$ and $y$ stands for the values of the holonomies along the other components of $\partial M_{1}$ and maybe also along other loops inside $M_{1}$.

The proof of this proposition is simlar to the proof of 5.1.2, with a small difference due to the consideration of particular gauge transformations. Note that 5.4 .2 implies the corresponding statement without any restrictions on the gauge transformations, which is in fact enough to 
prove the theorem 5.4.1. We will need this refinement in a forthcoming proof.

Proof of 5.4.2. We have

$$
\begin{aligned}
\int_{G^{\Gamma_{1}}} f d P^{\Gamma_{1}}\left(y, x, x^{-1}\right) & =\frac{1}{Z_{M_{1}}\left(y, x, x^{-1}\right)} \int_{G^{\Gamma_{1}}} f D^{\Gamma_{1}} d \nu_{y} d \nu_{x} d \nu_{x^{-1}} d g^{\prime} \\
& =\frac{1}{Z_{M_{1}}\left(y, x, x^{-1}\right)} \int_{G^{\Gamma_{1}, 2}} d \nu_{x} d \nu_{x^{-1}} \int_{G^{\Gamma_{1} \backslash \Gamma_{1}, \partial}} f D^{\Gamma_{1}} d \nu_{y} d g^{\prime} .
\end{aligned}
$$

The last integral is a function on $G^{\Gamma_{1, \partial}}$ and the same argument as in the proof of 5.1.2 together with the fact that we consider only gauge transformations such that $j\left(m_{1}\right)=j\left(m_{2}\right)=1$ shows that this function depends only on the values of $h_{L_{1}}$ and $h_{L_{2}}$. Thus, we can drop the integration against $d \nu_{x^{-1}}$ and replace the variables associated with the edges lying on $N_{2}$ by the variables associated to the corresponding edges on $N_{1}$. Thus,

$$
\begin{aligned}
\int_{G^{\Gamma_{1}}} f d P^{\Gamma_{1}}\left(y, x, x^{-1}\right) & =\frac{1}{Z_{M_{1}}\left(y, x, x^{-1}\right)} \int_{G^{\Gamma_{\partial}}} d \nu_{x} \int_{G^{\Gamma \backslash \Gamma_{\partial}}} f D^{\Gamma} d \nu_{y} d g^{\prime} \\
& =\frac{Z_{M}(y, x)}{Z_{M_{1}}\left(y, x, x^{-1}\right)} \int_{G^{\Gamma}} f d P^{\Gamma}(y, x) .
\end{aligned}
$$

The equality of the normalization constants follows from the case $f=1$ and finishes the proof.

Once again, we state separately the property of the conditional partition functions that we just established:

Proposition 5.4.3 For any $t_{1}, \ldots, t_{p}, t \in G / \mathrm{Ad}$, the following equality holds:

$$
Z_{M}\left(t_{1}, \ldots, t_{p}, t, t^{-1}\right)=Z_{M_{1}}\left(t_{1}, \ldots, t_{p}, t\right)
$$

Proof of 5.4.1. The proof is similar to that of the theorem 5.1.1, but simpler because there is essentially nothing more to prove than what is already stated in the proposition 5.4.2. By continuity of the random holonomy, it is enough to consider the case of a cylindrical function $f$ that depends on the holonomy along loops that can be put into a graph. So we choose such a function and consider a graph $\Gamma$ on $M$ such that $\Gamma^{*}$ contains all the loops with which we are working. Let $\Gamma_{1}$ be the graph on $M_{1}$ induced by $\Gamma$. The function $f$ induces a gauge-invariant function $f$ on $G^{\Gamma_{1}}$, and the equality which we need to prove about $f$ is exactly that given by the proposition 5.4.2.

As in the preceding situation, we want to know more about the inclusion of $\sigma$-algebras $\mathcal{A}_{1} \subset$ $\mathcal{A}$ and for this it is technically convenient to consider based loops: set $\Omega_{M}=\mathcal{F}\left(L_{m} M, G\right) / \operatorname{Ad}$ and $\Omega_{M_{1}}=\mathcal{F}\left(L_{m} M_{1}, G\right) /$ Ad, where $m=L(0)$. We identify $\mathcal{A}_{1}$ with the $\sigma$-algebra on $\Omega_{M}$ generated by the random holonomies along the loops of $i\left(L_{m} M\right)$.

Let us begin by a short informal discussion. Choose a path $c$ from $m_{1}$ to $m_{2}$. Although $c$ is an open path in $M_{1}, i(c)$ is a loop on $M$, so that the holonomy along $i(c)$ is well defined on $\Omega_{M}$ but certainly not $\mathcal{A}_{1}$-measurable. Once again, there is some information about $\mathcal{H}_{c}$ in $\mathcal{A}_{1}$ but there is also something missing, which is closely related to the centralizer of $H_{L}$. To see this, consider the random variable $\mathcal{H}_{L_{1}, c L_{2} c^{-1}}$. Since the holonomies along $L_{1}$ and $L_{2}$ must be equal after the gluing procedure, we choose arbitrarily an element $x \in t$ and compute as if 
$H_{L_{1}}=H_{L_{2}}=x$. The variable $\mathcal{H}_{L_{1}, c L_{2} c^{-1}}$, which is $\mathcal{A}_{1}$-measurable, determines to some extent the holonomy along $c$ : we interpret its value as that of $\left[x, H_{c}^{-1} x H_{c}\right]$ and this determines the value of $H_{c}$ up to left and right multiplications by elements of the centralizer $C(x)$.

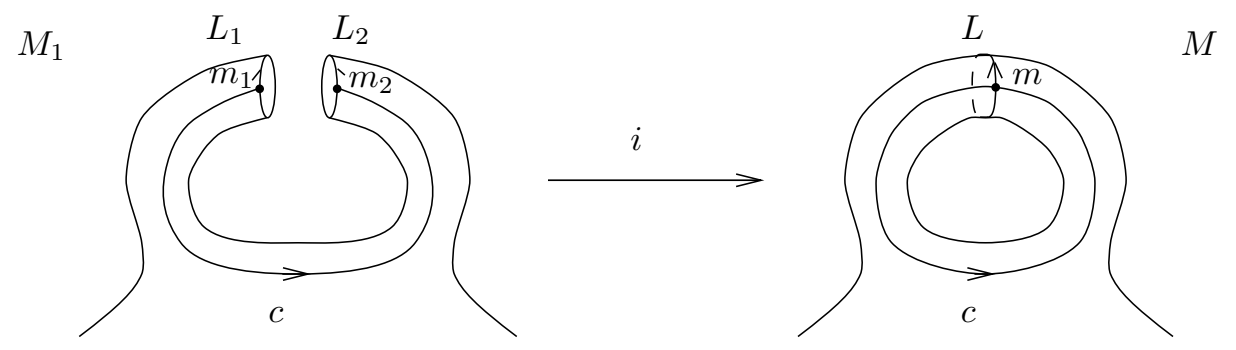

Figure 5.2: The new handle of $M$.

The main result is similar to the theorem 5.3.1:

Theorem 5.4.4 There exists a non-trivial sub- $\sigma$-algebra $\mathcal{Z}^{\prime}$ in $\mathcal{A}$ which is independent of $\mathcal{A}_{1}$ and such that the completions of $\mathcal{A}_{1} \vee \mathcal{Z}^{\prime}$ and $\mathcal{A}$ with respect to $\mu_{M}(u, t)$ are equal.

Note that the conclusion of this theorem does not depend on the fact that $G$ is Abelian or not. We will even see that, in some vague sense, $\mathcal{Z}^{\prime}$ is the biggest when $G$ is Abelian.

The proof of this theorem has the same structure as the proof of 5.3.1. We begin with a lemma which allows us to consider a restricted class of loops. We endow $M$ with a Riemannian metric such that $L$ is geodesic. This metric on $M$ induces by pull-back by $i$ a metric on $M_{1}$. We fix once for all a piecewise geodesic path $c$ in $M$ joining $m_{1}$ to $m_{2}$ and meeting $\partial M_{1}$ only at $m_{1}$ and $m_{2}$.

Lemma 5.4.5 On the space $\Omega_{M}$, the $\sigma$-algebra $\mathcal{A}$ is contained in the completion of the $\sigma$-algebra $\sigma\left(\mathcal{H}_{\lambda_{1}, \ldots, \lambda_{n}}, \lambda_{1}, \ldots, \lambda_{n} \in i\left(P G L_{m_{1}} M_{1} \cup\{c\}\right)\right)$ with respect to the measure $\mu_{M}(u, t)$.

Proof. Just as in the proof of 5.3.2, the point is to prove that any loop of $L_{m} M$ can be approximated by loops that are equivalent to finite products of loops of $i\left(P G L_{m_{1}} M_{1} \cup\{c\}\right)$. Consider a piecewise geodesic loop. Since it cuts at most a finite number of times $L$ transversally, it is equivalent to a finite product of loops of $P G L_{m} M$ that are the images by $i$ of loops based at $m_{1}$ or at $m_{2}$ or of paths with endpoints $m_{1}$ and $m_{2}$. Conjugation by $c$ or left or right multiplication by $c$ transform all these paths on $M_{1}$ into loops based at $m_{1}$, hence the holonomy along our piecewise geodesic loop can be expressed in terms of the holonomies along the path of $i\left(P G L_{m_{1}} M_{1} \cup\{c\}\right)$. Since any loop of $L_{m} M$ is a limit of piecewise geodesic loops, the result is proved.

Now, let us compute the conditional expectation of these variables with respect to $\mathcal{A}_{1}$. For this, we introduce a new notation, similar to the notation $\pi_{x}^{n}$ introduced in the preceding section.

Remark that the mapping $P G L_{m_{1}} M_{1} \cup\{c\} \longrightarrow P G L_{m} M$ induced by $i$ is injective, so that we regard $P G L_{m_{1}} M_{1} \cup\{c\}$ as a subset of $P G L_{m} M$.

Recall that $x$ is a fixed element in $t$ and consider an element of $\left(t \times t \times G^{n}\right) /$ Ad. It is a joint conjugacy class that can be written $\left[x, x_{c}^{-1} x x_{c}, x_{1}, \ldots, x_{n}\right]$. Let us compare two such 
representations of this class. If $\left[x, x_{c}^{-1} x x_{c}, x_{1}, \ldots, x_{n}\right]=\left[x, x_{c}^{\prime-1} x x_{c}^{\prime}, x_{1}^{\prime}, \ldots, x_{n}^{\prime}\right]$, then there exists $z_{1} \in C(x)$ such that $x_{i}^{\prime}=z_{1}^{-1} x_{i} z_{1}$ for $i=1, \ldots, n$ and $x_{c}^{\prime-1} x x_{c}^{\prime}=z_{1}^{-1} x_{c}^{-1} x x_{c} z_{1}$. This last equality implies that $x_{c} z_{1} x_{c}^{\prime-1}$ is an element $z_{2}$ of $C(x)$, so that $x_{c}^{\prime}=z_{2}^{-1} x_{c} z_{1}$. Thus, for any function $f$ on $G^{n+1} / \mathrm{Ad}$, the following integral is well defined:

$$
\int_{C(x)^{2}} f\left(z_{1}^{-1} x_{1} z_{1}, \ldots, z_{1}^{-1} x_{n} z_{1}, z_{2}^{-1} x_{c} z_{1}\right) d z_{1} d z_{2}=\int_{C(x)} f\left(x_{1}, \ldots, x_{n}, z x_{c}\right) d z .
$$

We denote by $\alpha_{x}^{n+1}\left(\left[x, x_{c}^{-1} x x_{c}, x_{1}, \ldots, x_{n}\right]\right)$ the corresponding measure on $G^{n+1} / \mathrm{Ad}$.

Proposition 5.4.6 Let $\lambda_{1}, \ldots, \lambda_{n}$ be loops of $P G L_{m_{1}} M_{1}$. For any continuous function $f$ on $G^{n+1} / A d$, we have

$$
E\left[f\left(\mathcal{H}_{\lambda_{1}, \ldots, \lambda_{n}, c}\right) \mid \mathcal{A}_{1}\right]=\int_{G^{n+1}} f d \alpha_{x}^{n+1}\left(\mathcal{H}_{L, c L c^{-1}, \lambda_{1}, \ldots, \lambda_{n}}\right) .
$$

Proof. Choose $l_{1}, \ldots, l_{m}$ in $P G L_{m_{1}} M_{1}$ and a continuous function $f_{1}$ on $G^{m} /$ Ad. Let $\Gamma$ be a graph on $M$ such that all the loops we are considering belong to $\Gamma^{*}$. Denote by $\Gamma_{1}$ the graph induced by $\Gamma$ on $M_{1}$, in the same way as we did in the proof of the theorem 5.4.1.

$$
\begin{aligned}
E & {\left[f\left(\mathcal{H}_{\lambda_{1}, \ldots, \lambda_{n}, c}\right) f_{1}\left(\mathcal{H}_{l_{1}, \ldots, l_{m}}\right)\right]=\int_{G^{\Gamma}} f\left(h_{\lambda_{1}}, \ldots, h_{\lambda_{n}}, h_{c}\right) f_{1}\left(h_{l_{1}}, \ldots, h_{l_{m}}\right) d P(y, x) } \\
& =\int_{G^{\Gamma} 1} f\left(h_{\lambda_{1}}, \ldots, h_{\lambda_{n}}, h_{c}\right) f_{1}\left(h_{l_{1}}, \ldots, h_{l_{m}}\right) d P^{\Gamma_{1}}\left(y, x, x^{-1}\right),
\end{aligned}
$$

thenks to 5.4.2. Indeed, the integrand here is not gauge-invariant because of the $h_{c}$, but it is precisely invariant by those gauge transformations $j$ such that $j\left(m_{1}\right)=j\left(m_{2}\right)=0$.

The end points of $h_{c}$ are now different and we can use the invariance of $P^{\Gamma_{1}}\left(y, x, x^{-1}\right)$ under a gauge transformation identically equal to 1 , except at $m_{2}$ where it takes the value $z^{-1} \in C(x)$. This transformation leaves the $h_{\lambda_{i}}$ 's and $h_{l_{i}}$ 's invariant since they are based at $m_{1}$, and transforms $h_{c}$ into $z h_{c}$. Thus,

$$
\begin{aligned}
& E\left[f\left(\mathcal{H}_{\lambda_{1}, \ldots, \lambda_{n}, c}\right) f_{1}\left(\mathcal{H}_{l_{1}, \ldots, l_{m}}\right)\right]= \\
= & \int_{G^{\Gamma_{1}}}\left[\int_{C(x)} f\left(h_{\lambda_{1}}, \ldots, h_{\lambda_{n}}, z h_{c}\right) d z\right] f_{1}\left(h_{l_{1}}, \ldots, h_{l_{m}}\right) d P^{\Gamma_{1}}\left(y, x, x^{-1}\right) \\
= & \int_{G^{\Gamma_{1}}}\left[\int_{G^{n+1}} f d \alpha_{x}^{n+1}\left(\left[h_{L}, h_{c L c^{-1}}, h_{\lambda_{1}}, \ldots, h_{\lambda_{n}}\right]\right)\right] f_{1}\left(h_{l_{1}}, \ldots, h_{l_{m}}\right) d P^{\Gamma_{1}}\left(y, x, x^{-1}\right) \\
= & E\left[\left(\int_{G^{n+1}} f d \alpha_{x}^{n+1}\left(\mathcal{H}_{L, c L c^{-1}, \lambda_{1}, \ldots, \lambda_{n}}\right)\right) f_{1}\left(\mathcal{H}_{l_{1}, \ldots, l_{m}}\right)\right]
\end{aligned}
$$

which implies the result.

It is time now to construct the random variable $Z^{\prime}$ that will generate the $\sigma$-algebra $\mathcal{Z}^{\prime}$. We will use the existence of the set $S_{N}$ and the section $\tau$ defined by the lemmas 5.3.4 and 5.3.5. We need also a new mapping $\sigma: t \longrightarrow G$ defined as follows. Let $g=h^{-1} x h$ be an element of $t$. The element $h$ is not uniquely defined by $g$, but only its class in $G / C(x)$. Then $\sigma$ is the composition of this well-defined map $t \longrightarrow G / C(x)$ with a measurable section $G / C(x) \longrightarrow G$. The existence of such a measurable section can be shown in the same way as we proved that $\tau$ exists. 
We fix now $N$ loops $L_{1}^{1}, \ldots, L_{N}^{1}$ in $P G L_{m_{1}} M_{1}$ such that the distribution of $\left(H_{L_{1}}, \ldots, H_{L_{N}}\right)$ has a density with respect to the Haar measure on $G^{N}$. Then $\mu_{M}(u, t)$-almost surely, this $N$-uple takes its values in $S_{N}$. Let $\omega \in \Omega_{M}$ be typical with this respect. Set $\tau\left(\mathcal{H}_{L, L_{1}^{1}, \ldots, L_{N}^{1}}\right)=$ $\left(x, x_{1}, \ldots, x_{N}\right)$ and choose $x_{c} \in G$ such that $\mathcal{H}_{L, L_{1}^{1}, \ldots, L_{N}^{1}, c}=\left[x, x_{1}, \ldots, x_{N}, x_{c}\right]$. Then $x_{c}$ is defined up to conjugation by an element of $C\left(x_{1}\right) \cap \ldots \cap C\left(x_{N}\right)=Z(G)$, so that it is in fact uniquely defined. Now, set $x_{c}^{\prime}=\sigma\left(x_{c}^{-1} x x_{c}\right)$. The product $x_{c} x_{c}^{\prime-1}$ belongs to $C(x)$ and we set

$$
Z^{\prime}(\omega)=x_{c} x_{c}^{\prime-1}
$$

Note that, in a similar fashion to $Z, Z^{\prime}$ depends on $\omega$ only through $\mathcal{H}_{L, L_{1}^{1}, \ldots, L_{N}^{1}, c}(\omega)$, via a function that we denote $\widetilde{Z}^{\prime}: G^{N+2} / \mathrm{Ad} \longrightarrow C(x)$. It is also easy to check that

$$
\widetilde{Z}^{\prime}\left(\left[x, x_{1}, \ldots, x_{N}, z x_{c}\right]\right)=z \widetilde{Z}^{\prime}\left(\left[x, x_{1}, \ldots, x_{N}, x_{c}\right]\right)
$$

holds for all $z$ in $C(x)$.

Proposition 5.4.7 The random variable $Z^{\prime}$ is independent of $\mathcal{A}_{1}$ and uniformly distributed on $C(x)$ under the measure $\mu_{M}(u, t)$.

In particular, $Z^{\prime}$ is never a constant random variable, since the centralizer of an element contains at least a one-parameter subgroup of $G$. If $G$ is Abelian, then $C(x)=G$, so that in this case, it is necessary to bring a uniform $G$-valued random variable in order to close a handle on $M$. This should be compared to the properties of the random holonomy along homologically non-trivial loops (see 1.9.4).

Proof. Let $f$ be a continuous function on $C(x)$. The function $Z^{\prime}$ can be seen as a function of the random variable $\mathcal{H}_{L, L_{1}^{1}, \ldots, L_{N}^{1}, c}$ and so will we do. Using 5.4.6, we get

$$
\begin{aligned}
E\left[f(Z) \mid \mathcal{A}_{1}\right] & =E\left[f\left(\widetilde{Z}^{\prime}\left(\mathcal{H}_{L, L_{1}^{1}, \ldots, L_{N}^{1}, c}\right)\right)\right] \\
& =\int_{G^{n+2}} f \circ \widetilde{Z}^{\prime} d \alpha_{x}^{n+2}\left(\mathcal{H}_{L, c L c^{-1}, L, L_{1}^{1}, \ldots, L_{N}^{1}}\right) .
\end{aligned}
$$

Let us fix $\omega$ and set $\left(x, x_{1}, \ldots, x_{N}\right)=\tau\left(\mathcal{H}_{L, L_{1}^{1}, \ldots, L_{N}^{1}}(\omega)\right)$. We assume that $C\left(x_{1}\right) \cap \ldots \cap$ $C\left(x_{N}\right)=Z(G)$. Let $x_{c}$ be the unique element of $G$ such that $\mathcal{H}_{L, L_{1}^{1}, \ldots, L_{N}^{1}, c}(\omega)=\left[x, x_{1}, \ldots, x_{N}, x_{c}\right]$. Then in particular, $\mathcal{H}_{L, c L c^{-1}, L, L_{1}^{1}, \ldots, L_{N}^{1}}=\left[x, x_{c}^{-1} x x_{c}, x_{1}, \ldots, x_{N}\right]$, so that by definition of $\alpha_{x}^{n+2}$,

$$
\begin{aligned}
E\left[f\left(Z^{\prime}\right) \mid \mathcal{A}_{1}\right](\omega) & =\int_{C(x)} f\left(\widetilde{Z}^{\prime}\left(x, x_{1}, \ldots, x_{N}, z x_{c}\right)\right) d z \\
& =\int_{C(x)} f\left(z \widetilde{Z}^{\prime}\left(x, x_{1}, \ldots, x_{N}, x_{c}\right)\right) d z \\
& =\int_{C(x)} f(z) d z .
\end{aligned}
$$

This conditional expectation is a constant for all $f$, hence the random variable $Z^{\prime}$ is independent of $\mathcal{A}_{1}$.

It remains to prove that the completion of $\mathcal{A}_{1} \vee \sigma\left(Z^{\prime}\right)$ contains $\mathcal{A}$. 
Lemma 5.4.8 The random variable $\mathcal{H}_{L, L_{1}^{1}, \ldots, L_{N}^{1}, c}$ is measurable with respect to the $\sigma$-algebra $\mathcal{A}_{1} \vee \sigma\left(Z^{\prime}\right)$.

PROOF. By construction of $Z^{\prime}$, the random variable $\mathcal{H}_{L, L_{1}^{1}, \ldots, L_{N}^{1}, c}$ is almost surely equal to

$$
\left[\tau\left(\mathcal{H}_{L, L_{1}^{1}, \ldots, L_{N}^{1}}\right), Z^{\prime} \sigma\left(\mathcal{H}_{c L c^{-1}}\right)\right]
$$

which is measurable with respect to $\mathcal{A}_{1} \vee \sigma\left(Z^{\prime}\right)$.

The next proposition finishes the proof of the theorem 5.4.4:

Theorem 5.4.9 The completion of the $\sigma$-algebra $\mathcal{A}_{1} \vee \sigma\left(\mathcal{H}_{L, L_{1}^{1}, \ldots, L_{N}^{1}, c}\right)$ with respect to the measure $\mu_{M}(t)$ contains $\mathcal{A}$.

Proof. Let $l_{1}, \ldots, l_{p}$ be $p$ loops of $i\left(P G L_{m_{1}} M_{1} \cup\{c\}\right)$. Since this proof is very close to that of 5.3.8, we do not write all details. The variables $\mathcal{H}_{c, L_{1}^{1}, \ldots, L_{N}^{1}}$ and $\mathcal{H}_{L_{1}^{1}, \ldots, L_{N}^{1}, l_{1}, \ldots, l_{p}}$ determine $\mathcal{H}_{c, l_{1}, \ldots, l_{p}}$ because they determine $\mathcal{H}_{c, L_{1}^{1}, \ldots, L_{N}^{1}, l_{1}, \ldots, l_{p}}$. This is proved using a map

$$
\left(G \times S_{N}\right) / \operatorname{Ad} \times_{S_{N}}\left(S_{N} \times G^{p}\right) / \operatorname{Ad} \stackrel{\sigma^{\prime}}{\longrightarrow}\left(G \times S_{N} \times G^{p}\right) / \operatorname{Ad} .
$$

It is easily checked that this map can be defined in such a way that $\mathcal{H}_{c, L, l}=\sigma^{\prime}\left(\mathcal{H}_{c, L}, \mathcal{H}_{L, l}\right)$. The fact that $\sigma^{\prime}$ is measurable terminates the proof.

\subsection{Conditional partition functions}

The propositions 5.1.3 and 5.4.3 show that the conditional partition functions deserve to be studied separately. We are interested in the conditional partition functions with respect to the boundary components of a surface. The importance of these functions had been pointed out by Witten Wi]. He already proved their algebraic properties using character expansions.

\subsubsection{Algebraic properties of the partition functions}

Let us summarize the properties of the conditional partition function that were proved at different points in the preceding chapters.

Let $(M, \sigma)$ be a surface, with a boundary $\partial M=N_{1} \cup \ldots \cup N_{p}$ or without boundary. For any graph $\Gamma$ on $M$ and any $x_{1}, \ldots, x_{p} \in G$, the number $\int_{G^{\Gamma}} D^{\Gamma} d \nu_{x_{1}} \ldots d \nu_{x_{p}} d g^{\prime}$ is well defined (see section 1.5). By the lemma 1.5.5, it depends only on the conjugacy classes of the $x_{i}$ 's: it is a central function of the $x_{i}$ 's. By the proposition 2.7.4, which is true on a surface with boundary by the lemma 2.8.2, this number does not depend on $\Gamma$. If $t_{1}=\left[x_{1}\right], \ldots, t_{p}=\left[x_{p}\right]$, we denote this number by $Z_{M}\left(t_{1}, \ldots, t_{p}\right)$, or just $Z_{M}$ if $M$ is closed.

Consider now an area-preserving diffeomorphism between $(M, \sigma)$ and another surface $\left(M^{\prime}, \sigma^{\prime}\right)$, i.e. a diffeomorphism that sends $\sigma$ to $\sigma^{\prime}$. Then an expression like $\int_{G^{\Gamma}} D^{\Gamma} d \nu_{x_{1}} \ldots d \nu_{x_{p}} d g^{\prime}$ is obviously invariant by this diffeomorphism. Thus, the function $Z_{M}$ depends on $M$ only through its class modulo area-preserving diffeomorphisms. As a consequence of Moser's theorem, that we extended to the case of surfaces with boundary in the proof of 2.2.1, this class is easily parametrized 
by a triple $(p, g, T) \in \mathbf{N}^{2} \times \mathbf{R}_{+}^{*}$, where $p$ is the number of components of $\partial M, g$ the genus of $M$ and $T$ the total surface of $M$. Another consequence of this invariance and of Moser's theorem is the symmetry of $Z_{M}$. Indeed, given any two components of $\partial M$, there exists a diffeomorphism of $M$ that permutes these components, hence an area-preserving diffeomorphism. Thus, for any $i$ and $j$ such that $1 \leq i<j \leq p, Z_{M}\left(t_{1}, \ldots, t_{i}, \ldots, t_{j}, \ldots, t_{p}\right)=Z_{M}\left(t_{1}, \ldots, t_{j}, \ldots, t_{i}, \ldots, t_{p}\right)$.

Let us give an expression of the function $Z_{M}$ that makes clear that it depends on $M$ only through $p, g, T$. We consider a graph with only one face on $M$, such that the boundary of this face is $\left[a_{1}, b_{1}\right] \ldots\left[a_{g}, b_{g}\right] c_{1}^{-1} N_{1} c_{1} \ldots c_{p}^{-1} N_{p} c_{p}$, where $a_{i}, b_{i}$ are the edges of a polygonal fundamental domain in the universal covering of $M$ and each $c_{i}$ joins $N_{i}$ to a point on the boundary of this fundamental domain. We find

$$
\begin{aligned}
Z_{p, g, T}\left(t_{1}, \ldots, t_{n}\right)= \\
=\int_{G^{2 g+p}} p_{\sigma(M)}\left(y_{1}^{-1} x_{1} y_{1} \ldots y_{p}^{-1} x_{p} y_{p}\left[a_{1}, b_{1}\right] \ldots\left[a_{g}, b_{g}\right]\right) d a_{1} d b_{1} \ldots d a_{g} d b_{g} d y_{1} \ldots d y_{p},
\end{aligned}
$$

where the $x_{i}$ 's are arbitrary representatives of the $t_{i}$ 's, and

$$
Z_{0, g, T}=\int_{G^{2 g+p}} p_{\sigma(M)}\left(\left[a_{1}, b_{1}\right] \ldots\left[a_{g}, b_{g}\right]\right) d a_{1} d b_{1} \ldots d a_{g} d b_{g}
$$

when $p=0$, i.e. when $M$ is closed. From now on, we index the function $Z$ by the triple $(p, g, T)$ instead of the surface $M$.

The expression 5.4 shows also that $Z_{p, g, T}$ is a smooth central function on $G^{p}$ and a continuous function on $(G / \mathrm{Ad})^{p}$. On the other hand, the symmetry of $Z_{p, g, T}$ is less obvious in this form. Using character expansions, it is possible to give a manifestly symmetric expression of $Z_{p, g, T}$. The reader which is not familiar with the characters of a compact Lie group should read the beginning of the section 4.2.2 before to go further. Using the expansion of the heat kernel proved in proposition 4.2.4, we transform (5.4) and (5.5) into:

$$
\begin{aligned}
Z_{p, g, T}\left(t_{1}, \ldots, t_{p}\right) & =\sum_{\alpha \in \widehat{G}}(\operatorname{dim} \alpha)^{2-2 g} e^{-\frac{c_{2}(\alpha)}{2} T} \prod_{i=1}^{p} \frac{\chi_{\alpha}\left(t_{i}\right)}{\operatorname{dim} \alpha}, \\
Z_{0, g, T} & =\sum_{\alpha \in \widehat{G}}(\operatorname{dim} \alpha)^{2-2 g} e^{-\frac{c_{2}(\alpha)}{2} T} .
\end{aligned}
$$

Before to state these results in a theorem, recall that $G / \mathrm{Ad}$ is endowed with the image measure of the Haar measure by the canonical projection $G \longrightarrow G$ /Ad. As we said at the beginning of this section, this theorem was essentially already proved by Witten.

Theorem 5.5.1 For each $(p, g, T) \in \mathbf{N}^{2} \times \mathbf{R}_{+}^{*}$, the function $Z_{g, p, T}$ is a continuous symmetric function on $(G / \mathrm{Ad})^{p}$. Moreover, for any $\left(p^{\prime}, g^{\prime}, T^{\prime}\right)$ and any $t_{1}, \ldots, t_{p}, t_{1}^{\prime}, \ldots, t_{p^{\prime}}^{\prime} \in G / \mathrm{Ad}$, the following relations hold:

$$
\begin{gathered}
\int_{G / \mathrm{Ad}} Z_{p+1, g, T}\left(t_{1}, \ldots, t_{p}, t\right) Z_{p^{\prime}+1, g^{\prime}, T^{\prime}}\left(t^{-1}, t_{1}^{\prime}, \ldots, t_{p^{\prime}}^{\prime}\right) d t= \\
Z_{p+p^{\prime}, g+g^{\prime}, T+T^{\prime}}\left(t_{1}, \ldots, t_{p}, t_{1}^{\prime}, \ldots, t_{p^{\prime}}^{\prime}\right), \\
\int_{G / \mathrm{Ad}} Z_{p+2, g, T}\left(t_{1}, \ldots, t_{p}, t, t^{-1}\right) d t=Z_{p, g+1, T}\left(t_{1}, \ldots, t_{p}\right) .
\end{gathered}
$$


Proof. The symmetry and continuity of $Z_{p, g, T}$ were already discussed. The relation (5.8) is a consequence of the proposition 5.1.3. Indeed, in this proposition, the number of components of the boundary of $M$ is $p_{1}+p_{2}-2$, where $p_{1}$ and $p_{2}$ are the number of components of $\partial M_{1}$ and $\partial M_{2}$ and its genus and total surface are the sums of those of $M_{1}$ and $M_{2}$. This gives:

$$
\begin{aligned}
\int_{G / \mathrm{Ad}} Z_{p+1, g, T}\left(t_{1}, \ldots, t_{p}, t\right) Z_{p^{\prime}+1, g^{\prime}, T^{\prime}}\left(t^{-1}, t_{1}^{\prime}, \ldots, t_{p^{\prime}}^{\prime}\right) d t= \\
\int_{G / \mathrm{Ad}} Z_{M}\left(t_{1}, \ldots, t_{p}, t, t_{1}^{\prime}, \ldots, t_{p^{\prime}}^{\prime}\right) d t .
\end{aligned}
$$

In this last partition function, the variable $t$ corresponds to an interior loop of $M$, not to a component of the boundary. If we compute this function using an expression like $\int_{G^{\Gamma}} D^{\Gamma} d \nu_{x} \ldots$, where $[x]=t$, we see that the conditioning with respect to this interior loop disappears if we integrate over $x$, so that the last integral is exactly equal to $Z_{p+p^{\prime}, g+g^{\prime}, T+T^{\prime}}\left(t_{1}, \ldots, t_{p}, t_{1}^{\prime}, \ldots, t_{p^{\prime}}^{\prime}\right)$.

Similarly, the relation (5.9) is a consequence of the proposition 5.4.3. In this gluing operation, the surface $M_{1}$ had lost two components of its boundary and gained one handle. Thus,

$$
\int_{G / \mathrm{Ad}} Z_{p+2, g, T}\left(t_{1}, \ldots, t_{p}, t, t^{-1}\right) d t=\int_{G / \mathrm{Ad}} Z_{M}\left(t_{1}, \ldots, t_{p}, t\right) d t .
$$

Just as above, $t$ disappears when we integrate against $d t$ because it corresponds to an interior loop and we find $Z_{p, g+1, T}\left(t_{1}, \ldots, t_{p}\right)$.

\subsubsection{Building bricks of the theory}

The two relations (5.8) and (5.9) are the analytic counterparts of the behaviour of the YangMills measure under the two basic surgery operations. It is well known that a few elementary surfaces are enough to build any surface by a sequence of these basic operations, namely a disk and a three-holed sphere (see fig. 5.3). It is not surprising that a corresponding result holds for the conditional partition functions.

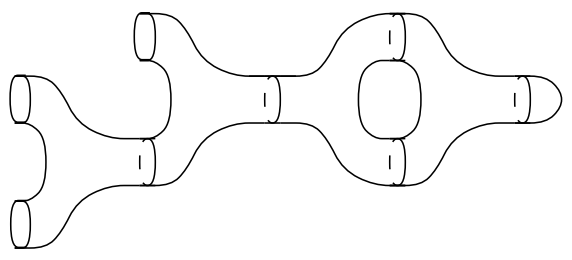

Figure 5.3: An example of decomposition in three-holed spheres and disks.

Proposition 5.5.2 The family of functions $Z_{p, g, T}$ is completely determined by the functions $Z_{1,0, T}$ and $Z_{3,0, T}, T>0$, and the relations (5.8) and (5.9).

Proof. We choose $g, p$ and construct the functions $Z_{g, p, T}, T>0$, starting with the functions $Z_{1,0, T}$ and $Z_{3,0, T}$.

Suppose first that $p+2 g \geq 3$. In this case, repeated applications of (5.8) to the function $Z_{3,0, T}$ allow to compute $Z_{p+2 g, 0, T}$ for any $T>0$. Now, $g$ applications of (5.9) to $Z_{p+2 g, 0, T}$ give the function $Z_{p, g, T}$. 
The case $p+2 g=2$ happens when $(p, g)=(0,1)$ or $(2,0)$. The first case is that of a closed torus. Start with a three-holed sphere and glue two components of its boundary. We get a torus with one hole. This corresponds to (5.9) applied to $Z_{3,0, T}$ to get $Z_{1,1, T}$. Now, it remains to glue a disk on the hole of the torus. In other words, the relation (5.8) applied to $Z_{1,1, T}$ and $Z_{1,0, T}$ gives $Z_{0,1, T}$. The case $(p, g)=(2,0)$ is that of a cylinder, which is obtained by gluing a disk on a three-holed sphere. So, $Z_{2,0, T}$ is obtained by applying (5.8) to $Z_{3,0, T}$ and $Z_{1,0, T}$.

The case $p+2 g=1$ happens only when $(p, g)=(1,0)$ and the corresponding function is one of our building bricks.

Finally, $p=g=0$ is a closed sphere, which can be obtained by gluing two disks together. So, (5.8) applied to $Z_{1,0, T}$ gives $Z_{0,0, T}$.

The natural question arising from this result is to identify the elementary functions $Z_{1,0, T}$ and $Z_{3,0, T}$.

Proposition 5.5.3 The function $Z_{1,0, T}$ is the projection on $G / \operatorname{Ad}$ of the heat kernel $p_{T}$ on $G$.

Proof. Any expression of $Z_{1,0, T}$, for example (5.4), proves this assertion.

The meaning of $Z_{3,0, T}$ is less obvious. Let us consider the functions $Z_{p, g, T}$ as functions on $G^{p}$ invariant by adjunction on each variable.

Lemma 5.5.4 For any $(p, g) \in \mathbf{N}^{2}$, any $T, T^{\prime}>0$, the following relation holds between central functions on $G$ :

$$
e^{T \frac{\Delta}{2}} Z_{p, g, T^{\prime}}\left(x_{1}, \ldots, x_{p-1}, \cdot\right)(x)=Z_{p, g, T+T^{\prime}}\left(x_{1}, \ldots, x_{p-1}, x\right) .
$$

In other words, $Z_{p, g, T}$ is a solution of the heat equation in each of its variables.

Proof. Given the fact that $\Delta \chi_{\alpha}=-c_{2}(\alpha) \chi_{\alpha}$ for any irreducible representation $\alpha$, this assertion is a simple consequence of 5.6 .

This lemma shows that the algebraic meaning of $Z_{3,0, T}$, if there is one, is contained in the formal $\operatorname{limit}_{\lim } \rightarrow 0 Z_{3,0, T}$. Let us look at a three-holed sphere with a very small surface (see fig. 5.4).

At the $T \rightarrow 0$ limit, there remains only two adjacent circles that form a graph. If we remind that the conditional partition function is the density of the natural law of the holonomy along the boundary of a surface (see 2.8.1), we see in this case that $Z_{3,0, T}$ is closely related to the multiplication in $G$.

Recall that the convolution product of two function $f, g \in L^{2}(G, d x)$ is defined by $f * g(x)=$ $\int_{G} f(y) g\left(y^{-1} x\right) d x$. It is also a square-integrable function. Let us denote by $L^{2}(G)^{G}$ the space of central $L^{2}$ functions on $G$. It is easily checked that the convolution product is a commutative operation in $L^{2}(G)^{G}$. Indeed, let $f$ and $g$ be two central functions. Using the left invariance of the Haar measure and its invariance by inversion, we get:

$$
f * g(x)=\int_{G} f(y) g\left(y^{-1} x\right) d y=\int_{G} f(x y) g\left(y^{-1}\right) d y=\int_{G} g(y) f\left(y^{-1} x\right) d y=g * f(x) .
$$



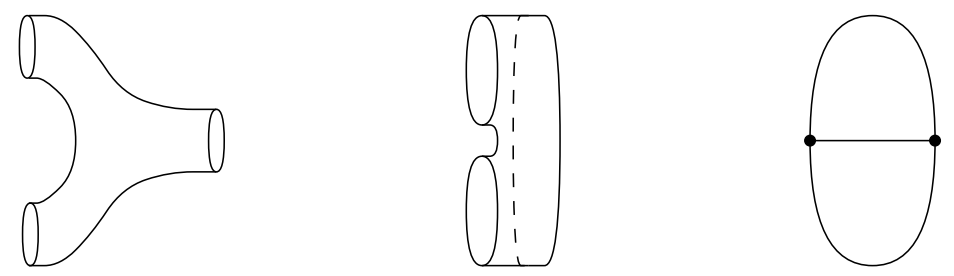

Figure 5.4: A thiner and thiner three-holed sphere.

It remains to check that $f * g$ is central.

$$
\begin{aligned}
& f * g(x y)=\int_{G} f(z) g\left(z^{-1} x y\right) d z=\int_{G} f(z) g\left(y z^{-1} x\right) d z= \\
& \int_{G} f(x z y) g\left(z^{-1}\right) d z=g * f(y x)
\end{aligned}
$$

and the result follows by commutativity of $*$.

This product on $L^{2}(G)^{G}$ is what remains from the product on $G$ when one considers conjugacy classes. The following result relates $Z_{3,0, T}$ to this product.

Proposition 5.5.5 Let $f$ and $g$ be two functions of $L^{2}(G)^{G}$. Then the following equality holds in $L^{2}(G)^{G}$ :

$$
\int_{G^{2}} f_{1}\left(x_{1}\right) f_{2}\left(x_{2}\right) Z_{3,0, T}\left(x_{1}, x_{2}, x\right) d x_{1} d x_{2}=\left[e^{-T \frac{\Delta}{2}}\left(f_{1} * f_{2}\right)\right](x) .
$$

This gives the interpretation that we were looking for: formally, $Z_{3,0,0}$ is the distributional kernel of the operator $*: L^{2}(G)^{G} \otimes L^{2}(G)^{G} \longrightarrow L^{2}(G)^{G}$. From this point of view, the commutativity of $*$ finds its geometric counterpart in the fact that two holes of a three-holed sphere are indistinguishable under area-preserving diffeomorphisms.

Proof. We use the fact that any central square-integrable function can be expanded into a series of characters. Thus, it is sufficient to prove the theorem when $f_{1}$ and $f_{2}$ are the characters of two irreducible representations $\alpha$ and $\beta$. We use the expansion of $Z_{3,0, T}$ given by (5.6).

$$
\begin{aligned}
\int_{G^{2}} \chi_{\alpha}\left(x_{1}\right) \chi_{\beta}\left(x_{2}\right) Z_{3,0, T}\left(x_{1}, x_{2}, x\right) d x_{1} d x_{2}= \\
=\sum_{\gamma \in \widehat{G}}(\operatorname{dim} \gamma)^{-1} e^{-\frac{c_{2}(\gamma)}{2} T} \chi_{\gamma}(x) \int_{G} \chi_{\alpha}\left(x_{1}\right) \chi_{\gamma}\left(x_{1}\right) d x_{1} \int_{G} \chi_{\beta}\left(x_{2}\right) \chi_{\gamma}\left(x_{2}\right) d x_{2} \\
=\sum_{\gamma \in \widehat{G}}(\operatorname{dim} \gamma)^{-1} e^{-\frac{c_{2}(\gamma)}{2} T} \chi_{\gamma}(x) \delta_{\alpha, \gamma} \delta_{\beta, \gamma} \\
=\delta_{\alpha, \beta} e^{-\frac{c_{2}(\alpha)}{2} T} \frac{\chi_{\alpha}(x)}{\operatorname{dim} \alpha}
\end{aligned}
$$

On the other hand, the orthogonality relations between characters imply:

$$
\chi_{\alpha} * \chi_{\beta}(x)=\int_{G} \chi_{\alpha}(y) \chi_{\beta}\left(y^{-1} x\right) d y=\delta_{\alpha, \beta} \frac{\chi_{\alpha}(x)}{\operatorname{dim} \alpha} .
$$


Finally, the fact that $\Delta \chi_{\alpha}=-c_{2}(\alpha) \chi_{\alpha}$ shows that the expression (5.10) is exactly equal to $\exp \left(T \frac{\Delta}{2}\right)\left(\chi_{\alpha} * \chi_{\beta}\right)$.

\subsubsection{Transition fonctions of the Markov field}

Consider the following very simple example. Take $M$ to be a cylinder $S^{1} \times[0,1]$ endowed with the Riemannian volume of the standard product metric, with total volume equal to 1 . Pick two elements $t_{0}$ and $t_{1}$ in $G /$ Ad and consider the Yang-Mills measure $\mu_{M}\left(t_{0}^{-1}, t_{1}\right)$.

For each $s \in[0,1]$, let $l_{s}$ be a loop whose image is the slice $S^{1} \times\{s\}$ in $M$. The family of random variables $\mathcal{H}_{l_{s}}, s \in[0,1]$ is a $G /$ Ad-valued process with index set $[0,1]$. The Markov property of the Yang-Mills field (theorem 5.1.1) implies that this process is a Markov process. Let us compute its transition functions. Choose $0<s_{1}<s_{2}<1$. Let $f_{1}$ and $f_{2}$ be two continuous functions on $G / \mathrm{Ad}$. We know by the proposition 1.5 .2 that

$$
E_{\mu_{M}\left(t_{0}^{-1}, t_{1}\right)}\left[f_{1}\left(\mathcal{H}_{l_{s_{1}}}\right) f_{2}\left(\mathcal{H}_{l_{s_{2}}}\right)\right]=\int_{(G / \mathrm{Ad})^{2}} f_{1}\left(u_{1}\right) f_{2}\left(u_{2}\right) \frac{Z_{M}\left(t_{0}^{-1}, u_{1}, u_{2}, t_{1}\right)}{Z_{2,0,1}\left(t_{0}^{-1}, t_{1}\right)} d u_{1} d u_{2}
$$

where the partition function in the integral is taken with respect to $l_{0}, l_{s_{1}}, l_{s_{2}}, l_{1}$. Using the proposition 5.1.3, we find that the expectation $E_{\mu_{M}\left(t_{0}, t_{1}\right)}\left[f_{1}\left(\mathcal{H}_{l_{s_{1}}}\right) f_{2}\left(\mathcal{H}_{l_{s_{2}}}\right)\right]$ is equal to

$$
\int_{(G / \mathrm{Ad})^{2}} f_{1}\left(u_{1}\right) f_{2}\left(u_{2}\right) \frac{Z_{2,0, s_{1}}\left(t_{0}^{-1}, u_{1}\right) Z_{2,0, s_{2}-s_{1}}\left(u_{1}^{-1}, u_{2}\right) Z_{2,0,1-s_{2}}\left(u_{2}^{-1}, t_{1}\right)}{Z_{2,0,1}\left(t_{0}^{-1}, t_{1}\right)} d u_{1} d u_{2} .
$$

Thus, the conditional expectation $E\left[f_{1}\left(\mathcal{H}_{l_{s_{1}}}\right) \mid \mathcal{H}_{l_{s_{2}}}\right]$ is equal to

\begin{tabular}{|c|c|c|c|}
\hline$l_{0}$ & $l_{s_{1}}$ & $l_{s_{2}}$ & $l_{1}$ \\
\hline & 1 & 1 & 1 \\
\hline & $i$ & $i$ & $i$ \\
\hline 1 & I & 1 & I \\
\hline & 1 & 1 & 1 \\
\hline & 1 & 1 & \\
\hline
\end{tabular}

$$
\frac{1}{Z_{2,0,1}\left(t_{0}^{-1}, t_{1}\right)} \int_{G / \mathrm{Ad}} f_{1}(u) Z_{2,0, s_{1}}\left(t_{0}^{-1}, u\right) Z_{2,0, s_{2}-s_{1}}\left(u^{-1}, \mathcal{H}_{l_{s_{2}}}\right) Z_{2,0,1-s_{2}}\left(\mathcal{H}_{l_{s_{2}}}^{-1}, t_{1}\right) d u \text {. }
$$

Figure 5.5: Transition functions on a cylinder.

The transition functions of the process are exactly the functions $Z_{2,0, T}$. This suggests that the functions $Z_{p, g, T}$ determine to some extent the law of the random holonomy. This was essentially the content of the proposition 1.5.2. More precisely, this proposition shows that it is possible to write down the law of the holonomy along a family of disjoint simple loops using only the partition functions. Using the continuity of the random holonomy, we can extend this statement a little bit. Indeed, let $l_{1}, \ldots, l_{n}$ be simple loops on $M$ that can be approximated by families of disjoint simple loops in such a way that none of the components of $M$ delimited by these families has a surface tending to 0 . Then the density of the law of the variable $\left(\mathcal{H}_{l_{1}}, \ldots, \mathcal{H}_{l_{n}}\right)$ 
is the limit of the densities of the holonomies along the approximating families. Since $Z_{p, g, T}$ depends continuously on its parameters and also on $T$ provided $T>0$, this limit density is also a combination of the functions $Z_{p, g, T}$.

Nevertheless, one cannot hope to express the law on $G^{2} /$ Ad of a variable like $\mathcal{H}_{l_{1}, l_{2}}$ using partition functions when $l_{1}$ and $l_{2}$ are based at the same point. Indeed, partition functions are functions on the space $(G / \mathrm{Ad})^{p}$ which is much smaller than the space $G^{p} / \mathrm{Ad}$. 


\section{Bibliography}

[Al] Albeverio, S., Høegh-Krohn, R., And Holden, H. Stochastic Lie group-valued measures and their relations to stochastic curve integrals, gauge fields and Markov cosurfaces. In Stochastic processes - mathematics and physics (Bielefeld, 1984). Springer, Berlin, 1986, pp. 1-24.

[AL] Ashtekar, A., And Lewandowski, J. Projective techniques and functional integration for gauge theories. J. Math. Phys. 36, 5 (1995), 2170-2191.

[AB] Atiyah, M. F., And Bott, R. The Yang-Mills equations over Riemann surfaces. Philos. Trans. Roy. Soc. London Ser. A 308, 1505 (1983), 523-615.

[Au] Aubin, T. Some nonlinear problems in Riemannian geometry. Springer-Verlag, Berlin, 1998.

[Be] Becker, C. Wilson loops in two-dimensional space-time regarded as white noise. J. Funct. Anal. 134, 2 (1995), 321-349.

[BS] Becker, C., And Sengupta, A. Sewing Yang-Mills measures and moduli spaces over compact surfaces. J. Funct. Anal. 152, 1 (1998), 74-99.

[BG] Berger, M., And Gostiaux, B. Géométrie différentielle. Librairie Armand Colin, Paris, 1972. Matrise de mathématiques, Collection U/Série "Mathématiques".

[Bl] Bleecker, D. Gauge theory and variational principles. Addison-Wesley Publishing Co., Reading, Mass., 1981.

[B1] Bourbaki, N. Éléments de mathématique. I: Les structures fondamentales de l'analyse. Fascicule VIII. Livre III: Topologie générale. Chapitre 9: Utilisation des nombres réels en topologie générale. Hermann, Paris, 1958. Deuxième édition revue et augmentée. Actualités Scientifiques et Industrielles, No. 1045.

[B2] Bourbaki, N. Éléments de mathématique. Fasc. XXVI. Groupes et algèbres de Lie. Chapitre I: Algèbres de Lie. Hermann, Paris, 1971. Seconde édition. Actualités Scientifiques et Industrielles, No. 1285.

[Br] Bröcker, T., And tom Dieck, T. Representations of compact Lie groups. SpringerVerlag, New York, 1995. Translated from the German manuscript, Corrected reprint of the 1985 translation.

[Ch] Chern, S.-S. An elementary proof of the existence of isothermal parameters on a surface. Proc. Amer. Math. Soc. 6 (1955), 771-782. 
[Ck] Choksi, J. R. Inverse limits of measure spaces. Proc. London Math. Soc. (3) 8 (1958), $321-342$.

[DM] Dellacherie, C., And Meyer, P.-A. Probabilities and potential. C. North-Holland Publishing Co., Amsterdam, 1988.

[dC] Do Carmo, M. P. A. Riemannian geometry. Birkhäuser Boston Inc., Boston, MA, 1992. Translated from the second Portuguese edition by Francis Flaherty.

[Dr1] Driver, B. K. Two-dimensional Euclidean quantized Yang-Mills fields. In Probability models in mathematical physics (Colorado Springs, CO, 1990). World Sci. Publishing, Teaneck, NJ, 1991, pp. 21-36.

[Dr2] Driver, B. K. YM $\mathrm{YM}_{2}$ : continuum expectations, lattice convergence, and lassos. Comm. Math. Phys. 123, 4 (1989), 575-616.

[Fi1] Fine, D. S. Quantum Yang-Mills on the two-sphere. Comm. Math. Phys. 134, 2 (1990), 273-292.

[Fi2] Fine, D. S. Quantum Yang-Mills on a Riemann surface. Comm. Math. Phys. 140, 2 (1991), 321-338.

[Fo] Forman, R. Small volume limits of 2-d Yang-Mills. Comm. Math. Phys. 151, 1 (1993), $39-52$.

[Gy] Gray, A. Tubes. Addison-Wesley Publishing Company Advanced Book Program, Redwood City, CA, 1990.

[Gr] Gross, L. A Poincaré lemma for connection forms. J. Funct. Anal. 63, 1 (1985), 1-46.

[GKS] Gross, L., King, C., And Sengupta, A. Two-dimensional Yang-Mills theory via stochastic differential equations. Ann. Physics 194, 1 (1989), 65-112.

[IW] Ikeda, N., AND Watanabe, S. Stochastic differential equations and diffusion processes, second ed. North-Holland Publishing Co., Amsterdam, 1989.

[KS] King, C., And Sengupta, A. The semiclassical limit of the two-dimensional quantum Yang-Mills model. J. Math. Phys. 35, 10 (1994), 5354-5361. Topology and physics.

[KN] Kobayashi, S., And Nomizu, K. Foundations of differential geometry. Vol. I. John Wiley \& Sons Inc., New York, 1996. Reprint of the 1963 original, A Wiley-Interscience Publication.

[Ku] Kuratowski, K. Topology. Vol. I. Academic Press, New York, 1966. New edition, revised and augmented. Translated from the French by J. Jaworowski.

[Liu] LiU, K. Heat kernel and moduli space. Math. Res. Lett. 3, 6 (1996), 743-762.

[Ma] Massey, W. S. A basic course in algebraic topology. Springer-Verlag, New York, 1991.

[Mi] Migdal, A. A. Recursion equations in gauge field theories. Sov. Phys. JETP 42, 3 (1975), 413-418. 
[Se1] Sengupta, A. The Yang-Mills measure for $S^{2}$. J. Funct. Anal. 108, 2 (1992), 231-273.

[Se2] Sengupta, A. Gauge theory on compact surfaces. Mem. Amer. Math. Soc. 126, 600 (1997), viii+85.

[Se3] Sengupta, A. Yang-Mills on surfaces with boundary: quantum theory and symplectic limit. Comm. Math. Phys. 183, 3 (1997), 661-705.

[Si] Simon, B. Representations of finite and compact groups. American Mathematical Society, Providence, RI, 1996.

[Va] Varopoulos, N. T., Saloff-Coste, L., and Coulhon, T. Analysis and geometry on groups. Cambridge University Press, Cambridge, 1992.

[Wi] Witten, E. On quantum gauge theories in two dimensions. Comm. Math. Phys. 141, 1 (1991), 153-209. 